\title{
Palladium-Catalyzed Solid-State Polyfluoroarylation of Aryl Halides Using Mechanochemistry
}

Rikuro Takahashi, ${ }^{\dagger}$ Tamae Seo, ${ }^{\dagger}$ Koji Kubota ${ }^{*}, \dagger, \sharp$ and Hajime Ito $*, \dagger, \sharp$

Division of Applied Chemistry, Graduate School of Engineering, Hokkaido University, Sapporo, Hokkaido 060-8628, Japan.

Institute for Chemical Reaction Design and Discovery (WPI-ICReDD), Hokkaido University, Sapporo, Hokkaido 060-8628, Japan.

e-mail:kbt@eng.hokudai.ac.jp, hajito@eng.hokudai.ac.jp

Table of Contents

1. Chemicals and Instrumentation

2. Substrate Synthesis

3. Set-Up Procedure for High-Temperature Ball-Milling Reactions

4. General Procedure for Solid-State Polyfluoroarylation

5. Thermography Images to Confirm Reaction Temperature

6. Kinetic Studies

7. Additional Data on Substrate Scope and Effect of Bases

8. Preliminary NMR Studies

9. Additional Mechanistic Studies

10. Single Crystal X-ray Structure Analyses

11. Calculation of E-Factors

12. Characterization of Obtained Coupling Products

13. References

14. NMR Spectra 


\section{Chemicals and Instrumentation}

The starting materials were obtained from commercial suppliers and used as received. Solvents were purchased from commercial suppliers, degassed via three freeze-pump-thaw cycles, and further

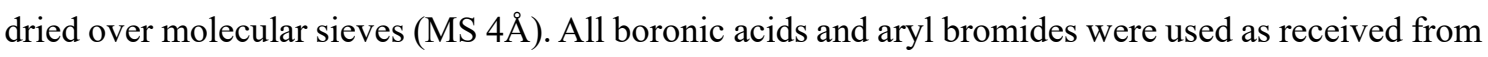
Tokyo Chemical Industry Co. (TCI), Sigma Aldrich, or Combi-Blocks. Tri(1-adamantyl)phosphine was obtained from Strem Chemicals, Inc. (product No. 15-0935) and used as received. Palladium precatalyst (4) was synthesized according to literature procedures. ${ }^{1,2}$ All mechanochemical reactions were carried out using grinding vessels in Retsch MM400 mill jars $(1.5 \mathrm{~mL})$, and balls are made of stainless (SUS440B and SUS420J2, respectively). The heat gun Takagi HG-1450B with temperature control function was used for the high-temperature ball-milling reactions. NMR spectra were recorded on JEOL JNM-ECX400P and JNM-ECS400 spectrometers ( ${ }^{1} \mathrm{H}$ : 392 or 396 or 399 or 400 or $401 \mathrm{MHz}$, ${ }^{13} \mathrm{C}$ : 99 or 100 or $151 \mathrm{MHz},{ }^{19} \mathrm{~F}: 369$ or 373 or $\left.376 \mathrm{MHz},{ }^{31} \mathrm{P}: 160 \mathrm{MHz}\right)$. Tetramethylsilane $\left({ }^{1} \mathrm{H}\right)$, $\mathrm{CDCl}_{3}\left({ }^{13} \mathrm{C}\right)$, benzotrifluoride $\left({ }^{19} \mathrm{~F}\right)$ were employed as external standards, respectively. Multiplicity was recorded as follows: $\mathrm{s}=$ singlet, $\mathrm{d}=$ doublet, $\mathrm{t}=$ triplet, $\mathrm{q}=$ quartet, $\mathrm{sept}=$ septet, $\mathrm{dd}=$ double doublet, $\mathrm{dt}=$ double triplet, $\mathrm{dq}=$ double quartet, $\mathrm{td}=$ triple doublet, $\mathrm{ddd}=$ double double doublet, quintd $=$ quinte doublet, $\mathrm{m}=$ multiplet, $\mathrm{dm}=$ double multiplet. Dibromomethane was used as an internal standard to determine NMR yields. Magic angle spinning (MAS) spectra were recorded on a Bruker MSL-300 spectrometer, operating at 202.5 MHz for 31P NMR (spinning rate $15 \mathrm{kHz}$ ). Recycle preparative gel permeation chromatography (GPC) was conducted with a JAI LaboACE LC-5060 or a JAI LC-9101 using $\mathrm{CHCl}_{3}$ as an eluent. Thermography was recorded with an NEC Avio Thermo GEAR G120. Single crystal X-ray structural analyses were carried out on a Rigaku XtaLAB PRO MM007 diffractometer using graphite monochromated $\mathrm{Cu}$-Ka radiation and PILATUS-200K detector. The structure was solved by direct methods and expanded using Fourier techniques. Non-hydrogen atoms were refined anisotropically. Hydrogen atoms were refined using the riding model. All calculations were performed using the Olex 2 crystallographic software package except for refinement, which was performed using SHELXL-2018. ${ }^{3}$ High-resolution mass spectra were recorded at the Global Facility Center, Hokkaido University. 


\section{Substrate Synthesis}

Preparation of pentafluorophenylboronic acid pinacol ester (2b)

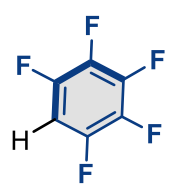

$10 \mathrm{mmol}$

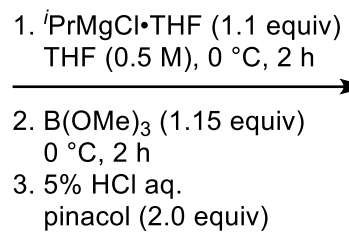

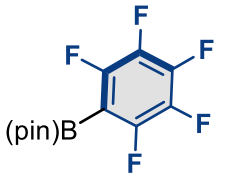

2b, $56 \%$

In a $50 \mathrm{~mL}$ round-bottomed flask, pentafluorobenzene $(1.71 \mathrm{~g}, 10 \mathrm{mmol})$ in dry THF $(14.5 \mathrm{~mL})$ was cooled to $0{ }^{\circ} \mathrm{C}$ under a nitrogen atmosphere. A THF solution of isopropyl magnesium chloride ( $5.5 \mathrm{ml}, 11.0 \mathrm{mmol}, 2.0 \mathrm{M}, 1.1$ equiv) was added dropwise. After $2 \mathrm{~h}$, trimethyl borate (1.3 mL, 1.15 equiv) was slowly added. The resulting mixture was allowed to stir at $0{ }^{\circ} \mathrm{C}$ for $2 \mathrm{~h}$. Then, the reaction mixture was quenched by the addition of $1 \mathrm{M} \mathrm{HCl}$ aqueous solution. The mixture was then extracted with pinacol (2.37 g, $20 \mathrm{mmol}, 2.0$ equiv) and $\mathrm{Et}_{2} \mathrm{O}$ three times dried over $\mathrm{MgSO}_{4}$, filtered, and concentrated by evaporation. The crude product was purified by flash column chromatography $\left(\mathrm{SiO}_{2}\right.$, $\mathrm{Et}_{2} \mathrm{O} /$ hexane, 0:100-10:90) to obtain $\mathbf{2 b}(1.66 \mathrm{~g}, 5.6 \mathrm{mmol}, 56 \%)$ as a white solid. 


\section{Set-Up Procedure for High-Temperature Ball-Milling Reactions}

A heat gun was fixed with clamps and placed directly above the ball milling jar (distance between the heat gun tip and the ball milling jar: ca. $1 \mathrm{~cm}$ ) (Figure S1). The set-up procedure for the hightemperature ball-milling reactions was shown in Figure S2. First, one grinding ball (stainless, diameter: $5 \mathrm{~mm}$ ) was loaded in a ball milling jar (stainless, $1.5 \mathrm{~mL}$ ). Then, materials were added to the jar. After the ball milling jar was closed, the jar was placed in the ball mill (Retsch MM400), and a heat gun was placed directly above the ball milling jar. The solid-state polyfluoroarylations were conducted while applying heated air outside the milling jar (the preset temperature at $250^{\circ} \mathrm{C}$ ).
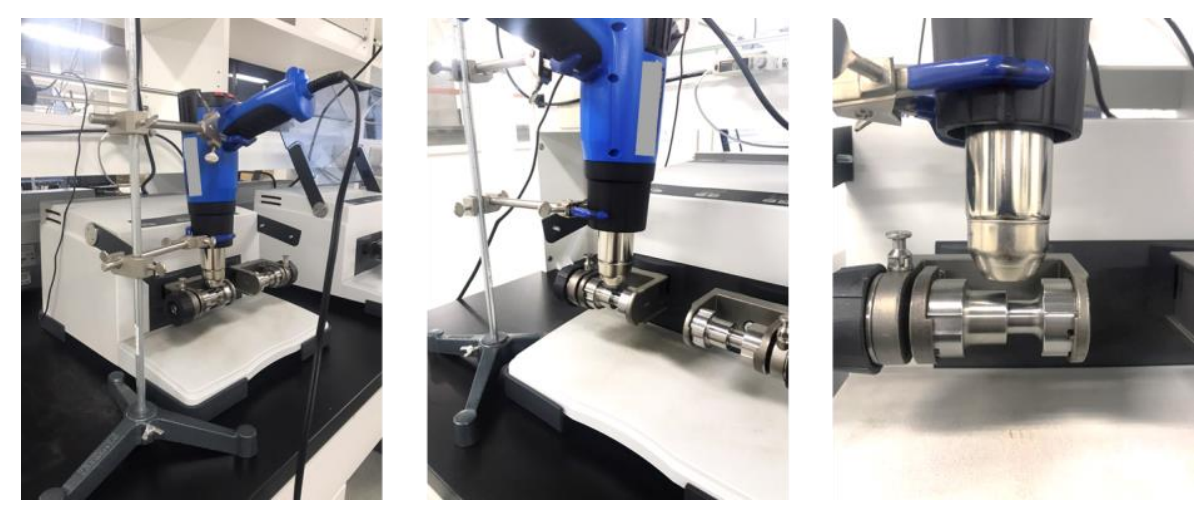

Figure S1. The set-up procedure for a heat gun on MM400.
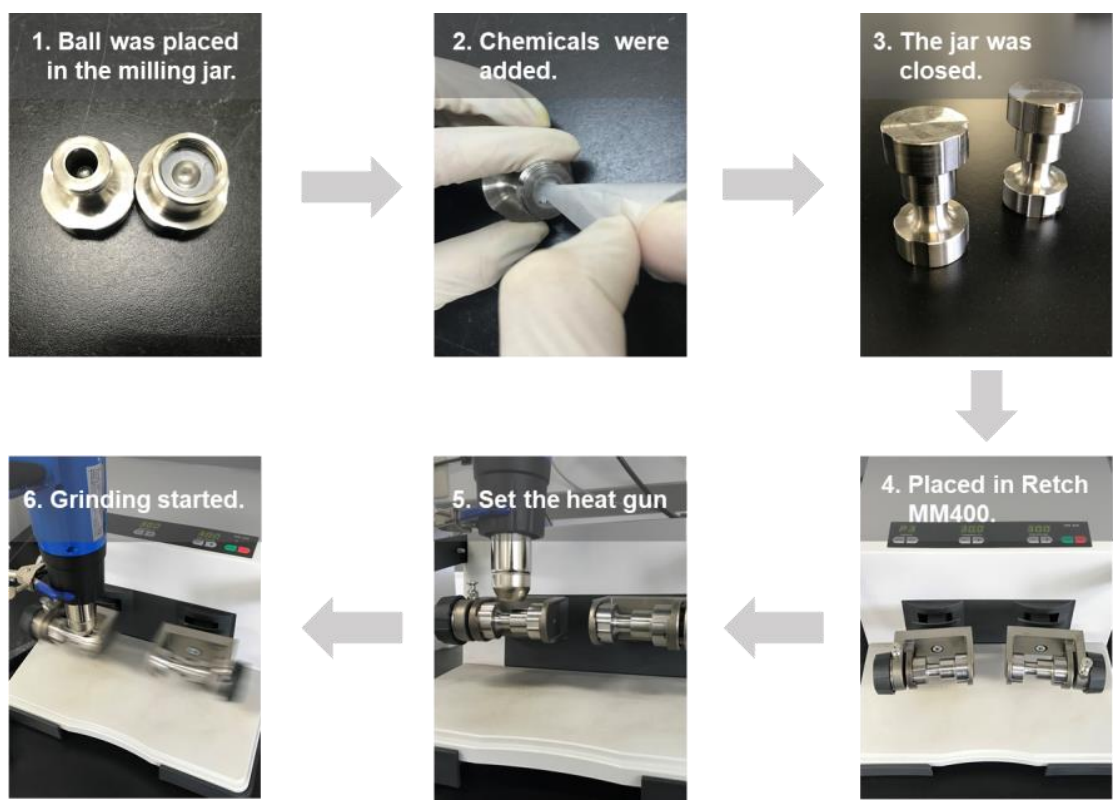

Figure S2. The set-up procedure for high-temperature ball-milling reactions. 


\section{General Procedure for Solid-State Polyfluoroarylation}

Procedure A: Mono-perfluoroarylation
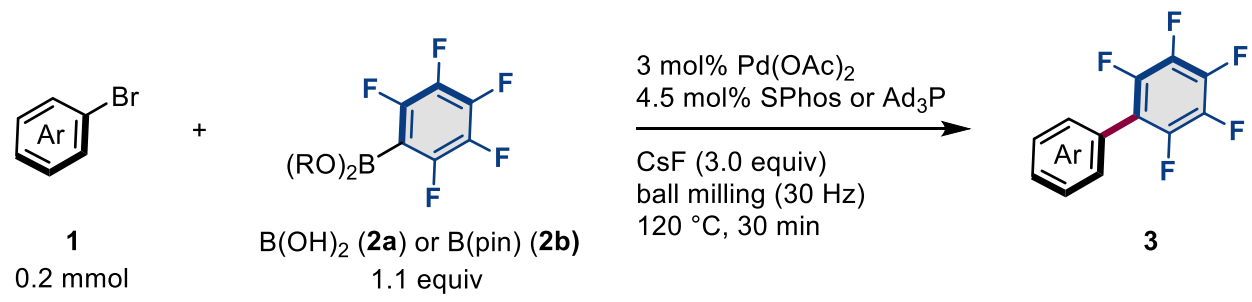

For reactions with 2a: aryl bromide $\mathbf{1}(0.2 \mathrm{mmol})$, aryl boronic acid $\mathbf{2 a}(0.22 \mathrm{mmol}, 1.1$ equiv), $\mathrm{Pd}(\mathrm{OAc})_{2}(0.006 \mathrm{mmol}, 3 \mathrm{~mol} \%)$ and SPhos or $\mathrm{Ad}_{3} \mathrm{P}(0.009 \mathrm{mmol}, 4.5 \mathrm{~mol} \%), \mathrm{CsF}(0.6 \mathrm{mmol}, 3.0$ equiv) were placed in a ball milling vessel (stainless, $1.5 \mathrm{~mL}$ ) loaded with one grinding ball (stainless, diameter: $5 \mathrm{~mm}$ ). After the vessel was closed in air, the vessel was placed in the ball mill (Retch MM400, 30Hz) and a heat gun (the preset temperature at $250{ }^{\circ} \mathrm{C}$ ). After ball milling for $30 \mathrm{~min}$, the mixture was passed through a short silica gel column $(15 \mathrm{~mm} \times 40 \mathrm{~mm})$ eluting with $\mathrm{CH}_{2} \mathrm{Cl}_{2}$ and EtOAc. The crude mixture was then purified by flash column chromatography $\left(\mathrm{SiO}_{2}\right.$, typically $\mathrm{CH}_{2} \mathrm{Cl}_{2} /$ hexane, typically 0:100-10:90) or reprecipitation from $\mathrm{CH}_{2} \mathrm{Cl}_{2} / \mathrm{MeOH}$ to give the corresponding coupling product 3 .

For reactions with $\mathbf{2 b}$ : aryl bromide $\mathbf{1}(0.2 \mathrm{mmol})$, aryl boronic acid $\mathbf{2 a}(0.22 \mathrm{mmol}, 1.1$ equiv), $\mathrm{Pd}(\mathrm{OAc})_{2}(0.006 \mathrm{mmol}, 3 \mathrm{~mol} \%)$ and SPhos or $\mathrm{Ad}_{3} \mathrm{P}$ (0.009 mmol, $\left.4.5 \mathrm{~mol} \%\right)$, CsF (0.6 mmol, 3.0 equiv) were placed in a ball milling vessel (stainless, $1.5 \mathrm{~mL}$ ) loaded with one grinding ball (stainless, diameter: $5 \mathrm{~mm}$ ). After the vessel was closed in air, the vessel was placed in the ball mill (Retch MM400, 30Hz) and a heat gun (the preset temperature at $250^{\circ} \mathrm{C}$ ). After ball milling for $30 \mathrm{~min}$, the mixture was extracted with $\mathrm{CH}_{2} \mathrm{Cl}_{2}$ three times. The combined organic layer was then dried over $\mathrm{MgSO}_{4}$. After filtration, the solvents were removed by evaporation. The crude mixture was then purified by flash column chromatography $\left(\mathrm{SiO}_{2}\right.$, typically $\mathrm{CH}_{2} \mathrm{Cl}_{2}$ /hexane, typically 0:100-10:90) or reprecipitation from $\mathrm{CH}_{2} \mathrm{Cl}_{2} / \mathrm{MeOH}$ to give the corresponding coupling product 3 .

\section{Procedure B: Double-perfluoroarylation}
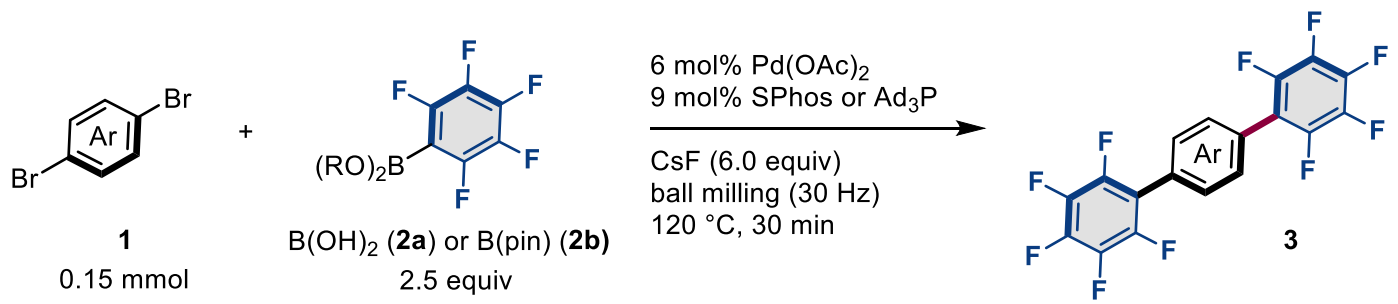

For reactions with 2a: aryl dibromide $\mathbf{1}(0.15 \mathrm{mmol})$, aryl boronic acid $\mathbf{2 a}(0.375 \mathrm{mmol}, 2.5$ equiv), $\mathrm{Pd}(\mathrm{OAc})_{2}(0.009 \mathrm{mmol}, 6 \mathrm{~mol} \%)$ and SPhos or $\mathrm{Ad}_{3} \mathrm{P}(0.0135 \mathrm{mmol}, 9 \mathrm{~mol} \%)$, CsF (0.9 mmol, 6.0 equiv) were placed in a ball milling vessel (stainless, $1.5 \mathrm{~mL}$ ) loaded with one grinding ball (stainless, diameter: $5 \mathrm{~mm}$ ). After the vessel was closed in air, the vessel was placed in the ball mill (Retch 
MM400, 30Hz) and a heat gun (the preset temperature at $250^{\circ} \mathrm{C}$ ). After ball milling for $30 \mathrm{~min}$, the mixture was passed through a short silica gel column $(15 \mathrm{~mm} \times 40 \mathrm{~mm})$ eluting with $\mathrm{CH}_{2} \mathrm{Cl}_{2}$ and EtOAc. The crude mixture was then purified by flash column chromatography $\left(\mathrm{SiO}_{2}\right.$, typically $\mathrm{CH}_{2} \mathrm{Cl}_{2}$ /hexane, typically 0:100-30:70) or reprecipitation from $\mathrm{CH}_{2} \mathrm{Cl}_{2} / \mathrm{MeOH}$ to give the corresponding coupling product 3 .

For reactions with $\mathbf{2 b}$ : aryl dibromide $\mathbf{1}(0.15 \mathrm{mmol})$, aryl boronic acid $\mathbf{2 b}(0.375 \mathrm{mmol}, 2.5$ equiv), $\mathrm{Pd}(\mathrm{OAc})_{2}(0.009 \mathrm{mmol}, 6 \mathrm{~mol} \%)$ and SPhos or $\mathrm{Ad}_{3} \mathrm{P}(0.0135 \mathrm{mmol}, 9 \mathrm{~mol} \%)$, CsF (0.9 mmol, 6.0 equiv) were placed in a ball milling vessel (stainless, $1.5 \mathrm{~mL}$ ) loaded with one grinding ball (stainless, diameter: $5 \mathrm{~mm}$ ). After the vessel was closed in air, the vessel was placed in the ball mill (Retch $\mathrm{MM} 400,30 \mathrm{~Hz}$ ) and a heat gun (the preset temperature at $250^{\circ} \mathrm{C}$ ). After ball milling for $30 \mathrm{~min}$, the mixture was extracted with $\mathrm{CH}_{2} \mathrm{Cl}_{2}$ three times. The combined organic layer was then dried over $\mathrm{MgSO}_{4}$. After filtration, the solvents were removed by evaporation. The crude mixture was then purified by flash column chromatography $\left(\mathrm{SiO}_{2}\right.$, typically $\mathrm{CH}_{2} \mathrm{Cl}_{2}$ /hexane, typically 0:100-30:70) or reprecipitation from $\mathrm{CH}_{2} \mathrm{Cl}_{2} / \mathrm{MeOH}$ to give the corresponding coupling product 3 .

\section{Procedure C: Double-perfluoroarylation with 1,5-cod}
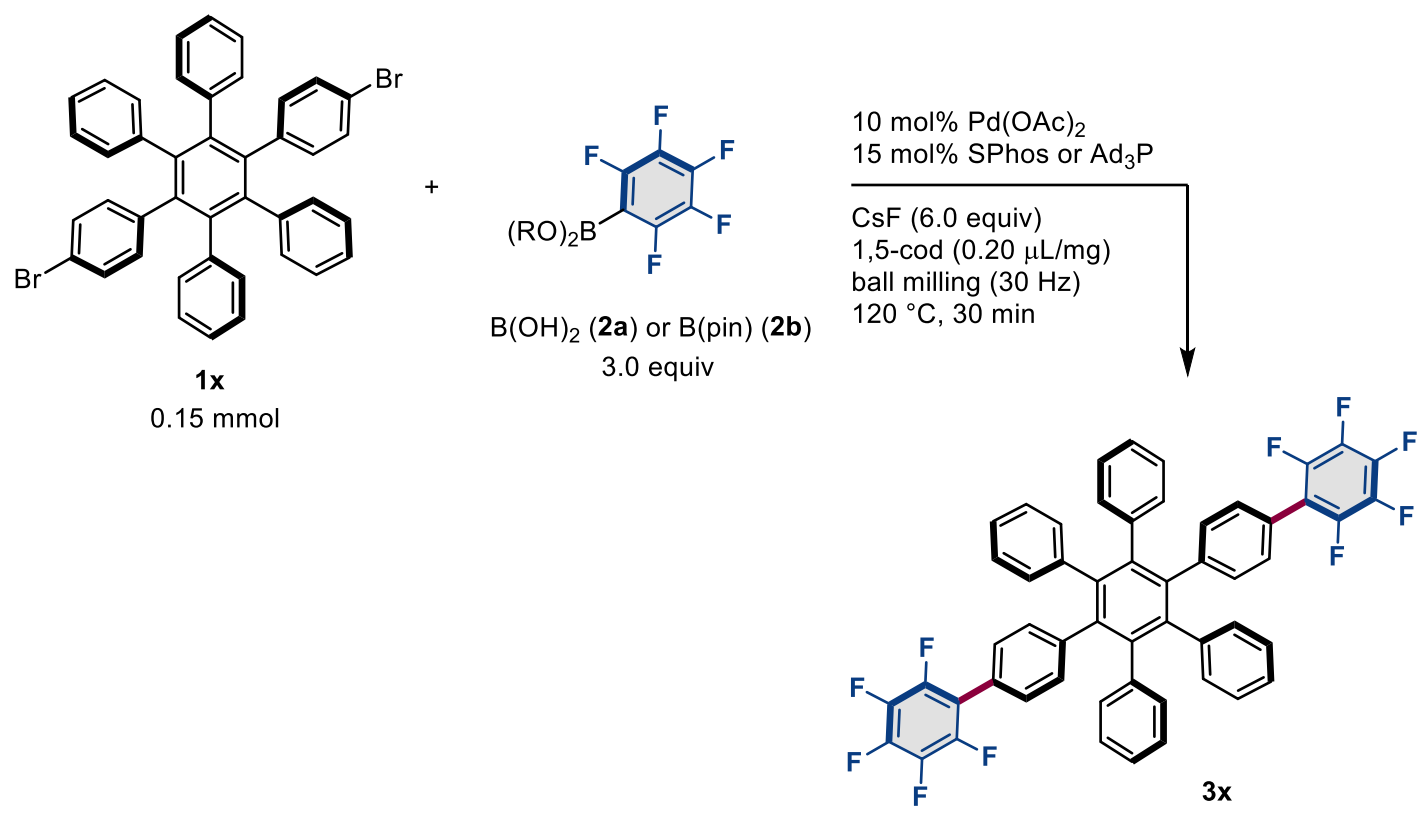

For reactions with 2a: 1x $(0.1 \mathrm{mmol})$, aryl boronic acid $\mathbf{2 a}\left(0.3 \mathrm{mmol}, 3.0\right.$ equiv), $\mathrm{Pd}(\mathrm{OAc})_{2}(0.01$ $\mathrm{mmol}, 10 \mathrm{~mol} \%)$ and SPhos or $\mathrm{Ad}_{3} \mathrm{P}(0.015 \mathrm{mmol}, 15 \mathrm{~mol} \%), \mathrm{CsF}(0.6 \mathrm{mmol}, 6.0$ equiv) were placed in a ball milling vessel (stainless, $1.5 \mathrm{~mL}$ ) loaded with one grinding ball (stainless, diameter: $5 \mathrm{~mm}$ ). Then 1,5-cod $(0.2 \mu \mathrm{L} / \mathrm{mg})$ was added via syringe. After the vessel was closed in air, the vessel was placed in the ball mill (Retch MM400,30Hz) and a heat gun (the preset temperature at $250{ }^{\circ} \mathrm{C}$ ). After ball milling for $60 \mathrm{~min}$, the mixture was passed through a short silica gel column $(15 \mathrm{~mm} \times 40 \mathrm{~mm})$ eluting with $\mathrm{CH}_{2} \mathrm{Cl}_{2}$ and EtOAc. The crude mixture was then purified by flash column chromatography 
( $\mathrm{SiO}_{2}, \mathrm{CH}_{2} \mathrm{Cl}_{2} /$ hexane, 0:100-30:70) and reprecipitation from $\mathrm{CH}_{2} \mathrm{Cl}_{2} / \mathrm{MeOH}$ to give the corresponding coupling product $\mathbf{3 x}$.

For reactions with $\mathbf{2 b} \mathbf{b} \mathbf{1 x}(0.1 \mathrm{mmol})$, aryl boronic acid $\mathbf{2 b}$ ( $0.3 \mathrm{mmol}, 3.0$ equiv), $\mathrm{Pd}(\mathrm{OAc})_{2}(0.01$ $\mathrm{mmol}, 10 \mathrm{~mol} \%)$ and SPhos or $\mathrm{Ad}_{3} \mathrm{P}(0.015 \mathrm{mmol}, 15 \mathrm{~mol} \%), \mathrm{CsF}(0.6 \mathrm{mmol}, 6.0$ equiv) were placed in a ball milling vessel (stainless, $1.5 \mathrm{~mL}$ ) loaded with one grinding ball (stainless, diameter: $5 \mathrm{~mm}$ ). Then $1,5-\operatorname{cod}(0.2 \mu \mathrm{L} / \mathrm{mg})$ was added via syringe. After the vessel was closed in air, the vessel was placed in the ball mill (Retch MM400, 30Hz) and a heat gun (the preset temperature at $250{ }^{\circ} \mathrm{C}$ ). After ball milling for $60 \mathrm{~min}$, the mixture was extracted with $\mathrm{CH}_{2} \mathrm{Cl}_{2}$ three times. The combined organic layer was then dried over $\mathrm{MgSO}_{4}$. After filtration, the solvents were removed by evaporation. The crude mixture was then purified by flash column chromatography $\left(\mathrm{SiO}_{2}, \mathrm{CH}_{2} \mathrm{Cl}_{2} /\right.$ hexane, 0:100-30:70) and reprecipitation from $\mathrm{CH}_{2} \mathrm{Cl}_{2} / \mathrm{MeOH}$ to give the corresponding coupling product $\mathbf{3 x}$.

\section{Procedure D: Tetra-perfluoroarylation}
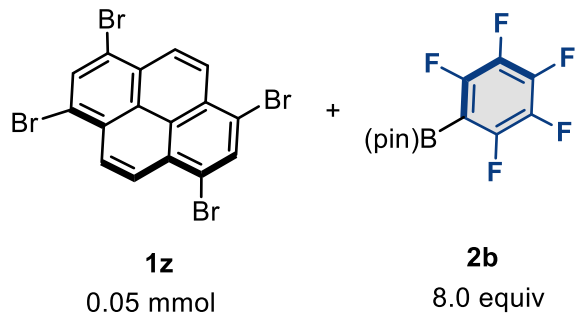

$15 \mathrm{~mol} \% \mathrm{Pd}(\mathrm{OAc})_{2}$ $22.5 \mathrm{~mol} \%$ SPhos

CsF (12.0 equiv) $1,5-\operatorname{cod}(0.20 \mu \mathrm{L} / \mathrm{mg})$ ball milling $(30 \mathrm{~Hz})$ $120^{\circ} \mathrm{C}, 30 \mathrm{~min}$

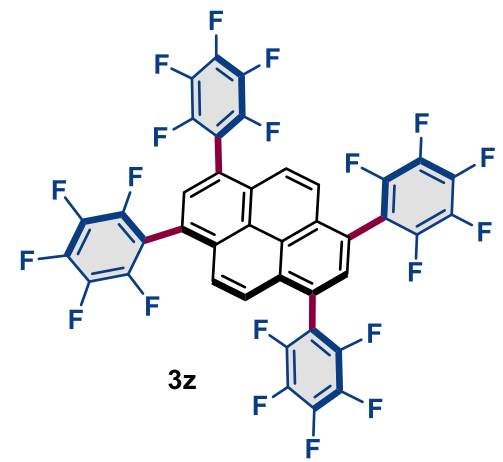

$\mathbf{1 z}(0.05 \mathrm{mmol})$, aryl boronic acid $\mathbf{2 b}\left(0.4 \mathrm{mmol}, 8.0\right.$ equiv), $\mathrm{Pd}(\mathrm{OAc})_{2}(0.0075 \mathrm{mmol}, 15 \mathrm{~mol} \%)$ and SPhos (0.0113 mmol, $22.5 \mathrm{~mol} \%), \mathrm{CsF}$ (0.6 mmol, 12.0 equiv) were placed in a ball milling vessel (stainless, $1.5 \mathrm{~mL}$ ) loaded with one grinding ball (stainless, diameter: $5 \mathrm{~mm})$. Then 1,5-cod $(0.2$ $\mu \mathrm{L} / \mathrm{mg}$ ) was added via syringe. After the vessel was closed in air, the vessel was placed in the ball mill (Retch MM400, 30Hz) and a heat gun (the preset temperature at $250{ }^{\circ} \mathrm{C}$ ). After ball milling for 30 min, the mixture was extracted with $\mathrm{CH}_{2} \mathrm{Cl}_{2}$ three times. The combined organic layer was then dried over $\mathrm{MgSO}_{4}$. After filtration, the solvents were removed by evaporation. The crude mixture was then purified by flash column chromatography $\left(\mathrm{SiO}_{2}, \mathrm{CH}_{2} \mathrm{Cl}_{2} /\right.$ hexane, 0:100-30:70) and GPC to give the corresponding coupling product $\mathbf{3 z}$. 
Procedure E: Cross-coupling reactions with other boronic acids

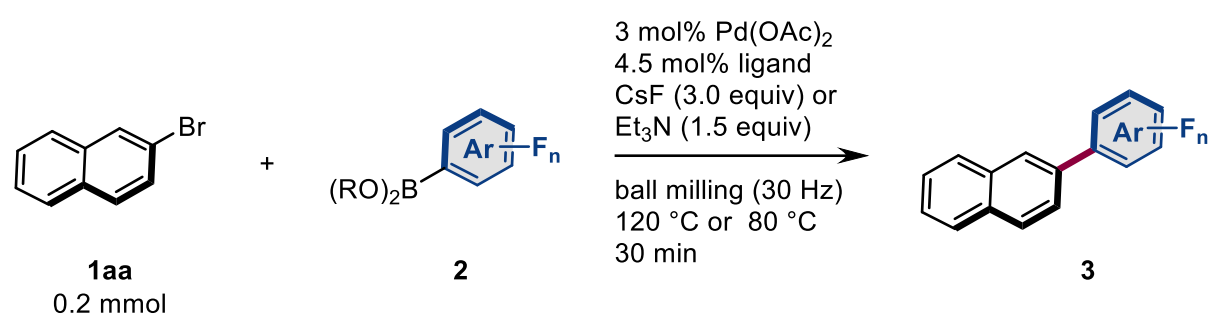

Aryl halide 1aa $(0.2 \mathrm{mmol})$, aryl boronic acid 2 ( 0.22 or $0.3 \mathrm{mmol}, 1.1$ or 1.5 equiv), $\mathrm{Pd}(\mathrm{OAc})_{2}$ (0.006 mmol, $3 \mathrm{~mol} \%)$ and SPhos ( $0.0075 \mathrm{mmol}, 4.5 \mathrm{~mol} \%), \mathrm{Et}_{3} \mathrm{~N}(0.3 \mathrm{mmol}, 1.5$ equiv) were placed in a ball milling vessel (stainless, $1.5 \mathrm{~mL}$ ) loaded with one grinding ball (stainless, diameter: $5 \mathrm{~mm}$ ). After the vessel was closed in air, the vessel was placed in the ball mill (Retch MM400, 30Hz) and a heat gun (the preset temperature at 250 or $150{ }^{\circ} \mathrm{C}$ ). After ball milling for $30 \mathrm{~min}$, the mixture was extracted with $\mathrm{CH}_{2} \mathrm{Cl}_{2}$ three times. The combined organic layer was then dried over $\mathrm{MgSO}_{4}$. After filtration, the solvents were removed by evaporation. The crude mixture was then purified by flash column chromatography ( $\mathrm{SiO}_{2}$, typically $\mathrm{CH}_{2} \mathrm{Cl}_{2} /$ hexane, typically 0:100-30:70) to give the corresponding coupling product $\mathbf{3}$.

\section{Procedure F: Cross-coupling reactions with water as additives}

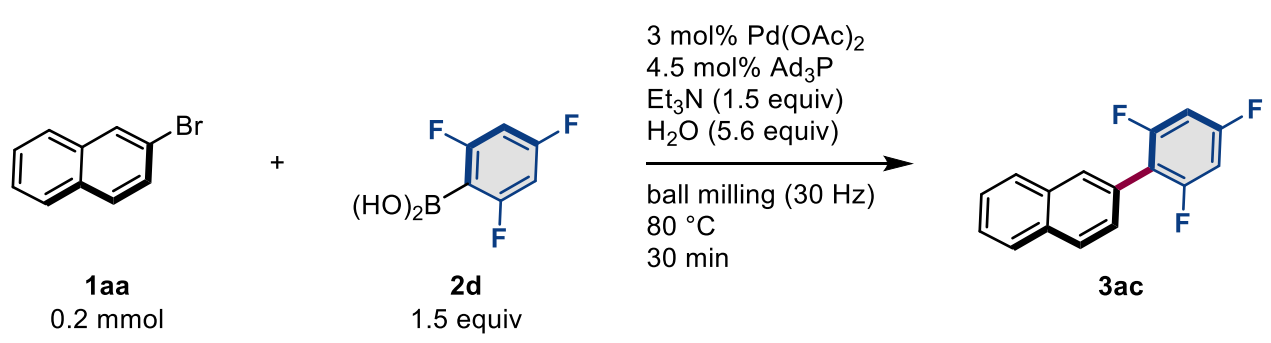

Aryl halide 1aa $(0.2 \mathrm{mmol})$, aryl boronic acid $\mathbf{2 d}\left(0.3 \mathrm{mmol}, 1.5\right.$ equiv), $\mathrm{Pd}(\mathrm{OAc})_{2}(0.006 \mathrm{mmol}$, $3 \mathrm{~mol} \%$ ) and SPhos ( $0.0075 \mathrm{mmol}, 4.5 \mathrm{~mol} \%), \mathrm{Et}_{3} \mathrm{~N}$ ( $0.3 \mathrm{mmol}, 1.5$ equiv), $\mathrm{H}_{2} \mathrm{O}$ (1.1 mmol, 5.6 equiv) were placed in a ball milling vessel (stainless, $1.5 \mathrm{~mL}$ ) loaded with one grinding ball (stainless, diameter: $5 \mathrm{~mm}$ ). After the vessel was closed in air, the vessel was placed in the ball mill (Retch $\mathrm{MM} 400,30 \mathrm{~Hz}$ ) and a heat gun (the preset temperature at $150^{\circ} \mathrm{C}$ ). After ball milling for $30 \mathrm{~min}$, the mixture was extracted with $\mathrm{CH}_{2} \mathrm{Cl}_{2}$ three times. The combined organic layer was then dried over $\mathrm{MgSO}_{4}$. After filtration, the solvents were removed by evaporation. The crude mixture was then purified by flash column chromatography $\left(\mathrm{SiO}_{2}\right.$, hexane only) to give the corresponding coupling product 3ac. 


\section{Thermography Images to Confirming Reaction Temperature}

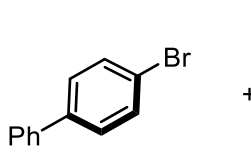

$1 \mathrm{a}$

$0.2 \mathrm{mmol}$

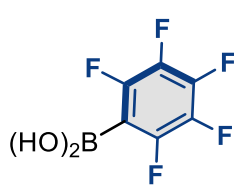

2a

1.1 equiv

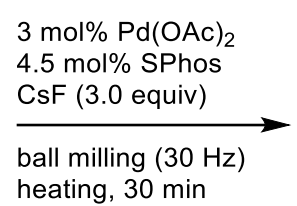

heating, $30 \mathrm{~min}$

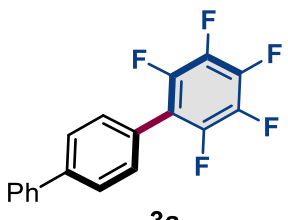

$3 a$

The temperature inside the milling jar after the solid-state polyfluoroarylation was checked by thermography measurement immediately after opening the milling jar. The crude mixtures were prepared by the following conditions: $0.2 \mathrm{mmol}$ of $\mathbf{1 a} ; 0.22 \mathrm{mmol}$ of $\mathbf{2 a} ; 0.006 \mathrm{mmol}$ of $\mathrm{Pd}(\mathrm{OAc})_{2}$; $0.009 \mathrm{mmol}$ of the ligand; $0.6 \mathrm{mmol}$ of $\mathrm{CsF}$ in a stainless ball milling jar (SUS440B, $1.5 \mathrm{~mL}$ ) with one stainless-steel ball (SUS420J2, $5 \mathrm{~mm}$ ); $30 \mathrm{~Hz}$; heat gun; $30 \mathrm{~min}$.

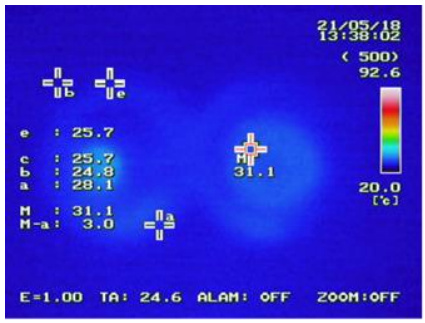

no heating

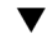

internal temp. : $31.1^{\circ} \mathrm{C}$
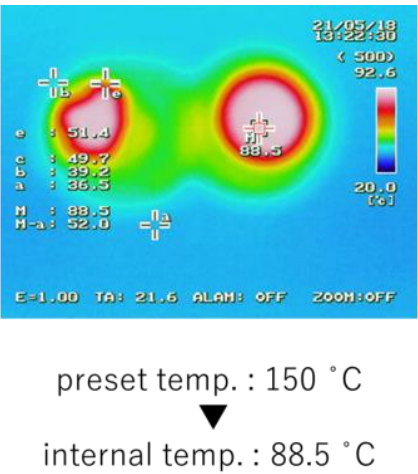
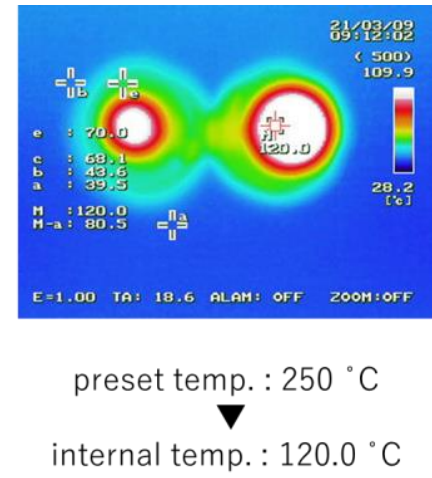

Figure S3. Thermography images of the reaction mixtures after the ball milling at $30 \mathrm{~Hz}$ for $30 \mathrm{~min}$ with or without heating. 


\section{Kinetic Studies}

Kinetics study of the polyfluoroarylation using $2 \mathrm{a}$ at different concentrations

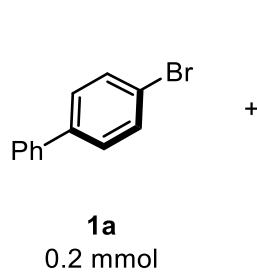

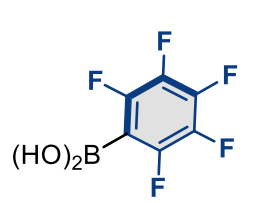

$2 a$

1.1 equiv

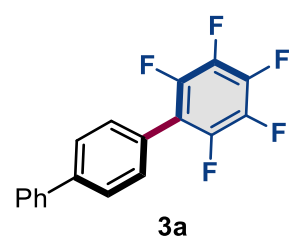

GC yield (\%)

The kinetic study on the reactions with $\mathbf{2 a}$ was conducted under solid-state conditions and in solution at different concentrations (Figure S4). All sampling data for mechanochemsitry were measured individually because the mechanochemical reactions require stopping the mill and opening jar,. The solution-based reactions are carried out in reflux state at $120^{\circ} \mathrm{C}$. The yields were determined by GC analysis with tridecane as an internal standard. Under the conventional solution-based conditions, the reaction rate increased slightly with the increasing concentration of the substrates and the catalyst ( $0.1 \mathrm{M}$ to $1.5 \mathrm{M}$ ), albeit that the yield of 3a remained below $20 \%$ in all cases. In contrast, using the developed solid-state conditions, the reaction completed rapidly (within $30 \mathrm{~min}$ ) and $\mathbf{3 a}$ was obtained in quantitative yield.

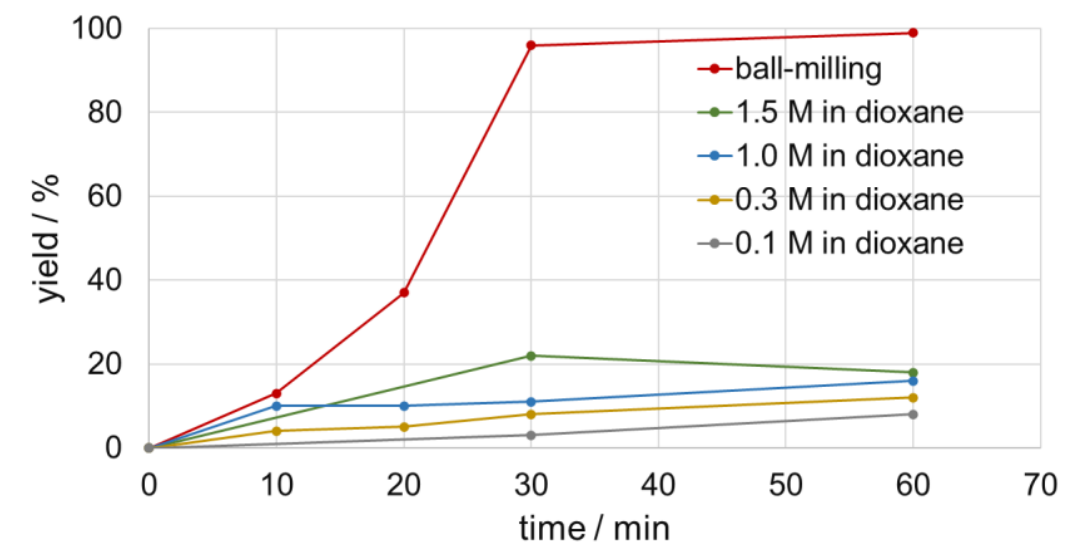

Figure S4 Results of kinetics study on the reaction using $\mathbf{2 a}$.

Table S1. Results of kinetics study using $\mathbf{2 a}$.

\begin{tabular}{cccccc}
\hline time & $\begin{array}{c}0.1 \mathrm{M} \\
\text { in dioxane }\end{array}$ & $\begin{array}{c}0.3 \mathrm{M} \\
\text { in dioxane }\end{array}$ & $\begin{array}{c}1.0 \mathrm{M} \\
\text { in dioxane }\end{array}$ & $\begin{array}{c}1.5 \mathrm{M} \\
\text { in dioxane }\end{array}$ & ball-milling \\
\hline $10 \mathrm{~min}$ & - & $4 \%$ & $10 \%$ & - & $13 \%$ \\
$20 \mathrm{~min}$ & - & $5 \%$ & $10 \%$ & - & $37 \%$ \\
$30 \mathrm{~min}$ & $3 \%$ & $8 \%$ & $11 \%$ & $22 \%$ & $96 \%$ \\
$60 \mathrm{~min}$ & $8 \%$ & $12 \%$ & $16 \%$ & $18 \%$ & $99 \%$ \\
$120 \mathrm{~min}$ & $17 \%$ & $11 \%$ & $23 \%$ & $25 \%$ & - \\
$240 \mathrm{~min}$ & $34 \%$ & $31 \%$ & $29 \%$ & $27 \%$ & - \\
\hline
\end{tabular}




\section{Kinetics studies of the polyfluoroarylation using $2 \mathrm{f}$ at different concentrations}
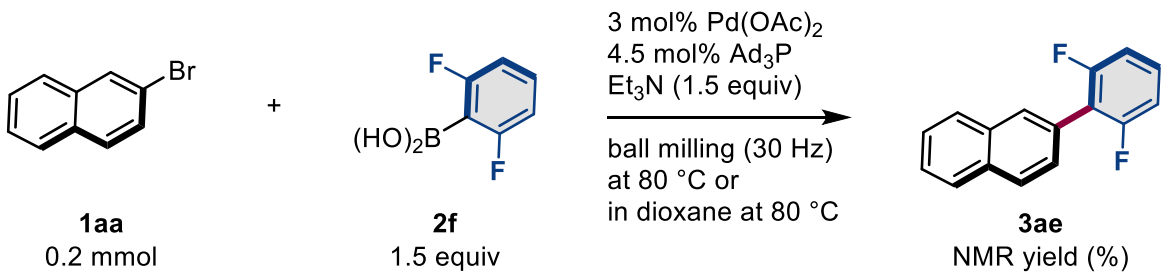

The kinetic study on the reactions with $\mathbf{2 f}$ was conducted under solid-state conditions and in solution at different concentrations (Figure S4). All sampling data of the mechanochemical conditions were measured individually because the mechanochemical reactions require stopping the mill and opening jar. The yields were determined by ${ }^{19} \mathrm{~F}$ NMR analysis with benzotrifluoride as an internal standard. We observed an increasing reaction rate upon increasing the concentration from $0.3 \mathrm{M}$ to 1.0 M under the solution-based conditions. Under our solid-state conditions, the cross-coupling was very fast (complete within $10 \mathrm{~min}$ ) to form 3ae in quantitative yield.

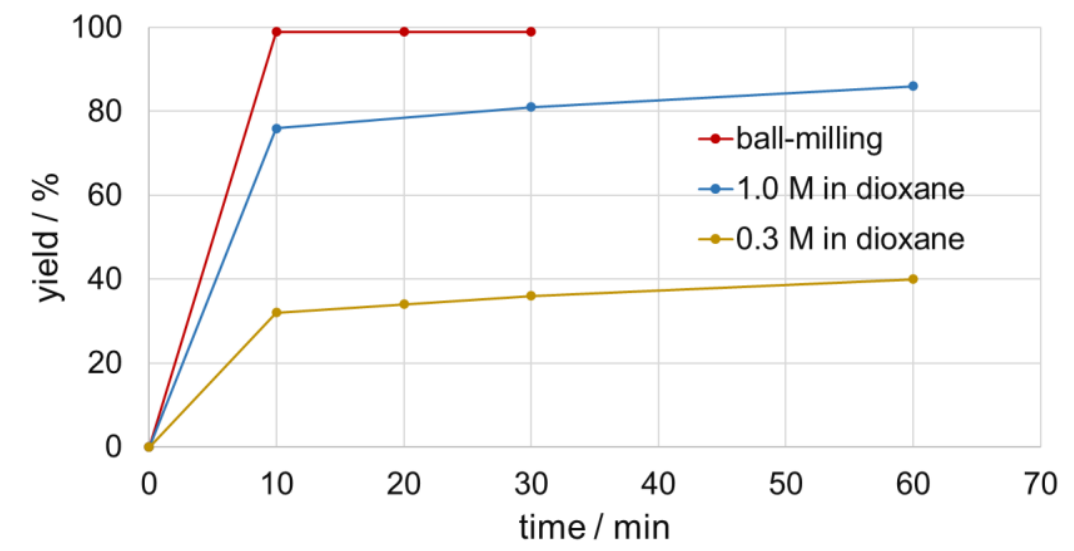

Figure S5. Results of kinetics study using $\mathbf{2 f}$.

Table S2. Results of kinetics study using $2 \mathbf{f}$.

\begin{tabular}{cccc}
\hline time & $\begin{array}{c}0.3 \mathrm{M} \\
\text { in dioxane }\end{array}$ & $\begin{array}{c}1.0 \mathrm{M} \\
\text { in dioxane }\end{array}$ & ball-milling \\
\hline $10 \mathrm{~min}$ & $32 \%$ & $76 \%$ & $99 \%$ \\
$20 \mathrm{~min}$ & $34 \%$ & - & $99 \%$ \\
$30 \mathrm{~min}$ & $36 \%$ & $81 \%$ & $99 \%$ \\
$60 \mathrm{~min}$ & $40 \%$ & $86 \%$ & - \\
$120 \mathrm{~min}$ & $43 \%$ & $89 \%$ & - \\
$240 \mathrm{~min}$ & $48 \%$ & $89 \%$ & - \\
\hline
\end{tabular}




\section{Additional Data on Substrate Scope and Effect of Bases}

\section{Investigation of the effect of substituents at the ortho-position}

We investigated additional substrates with substituents such as $-\mathrm{Et}$, $-\mathrm{OMe}$, and $-\mathrm{CO}_{2} \mathrm{Me}$ at the ortho-position, which afforded the desired products in low to moderate yield. These results were summarized as Table S3 shown below.

Table S3. Scope of aryl bromides that contain a variety of substituents at the ortho-position.

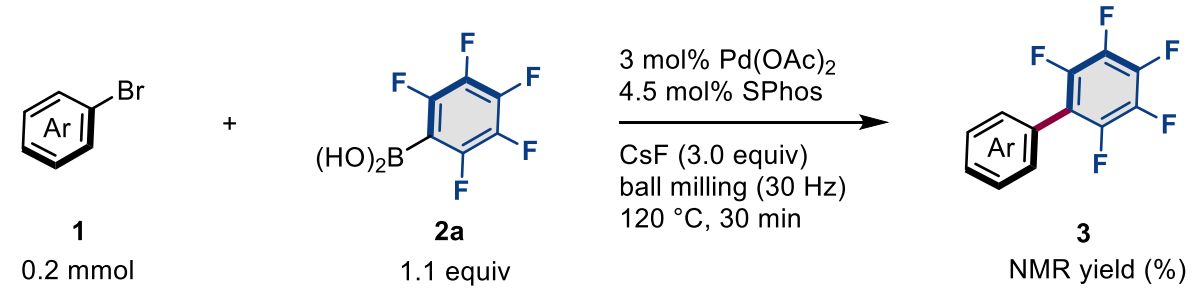

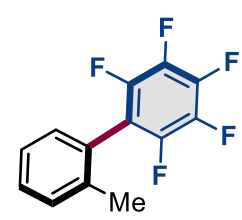

3d: $30 \%$

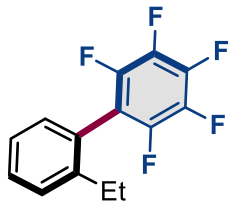

S3a: $26 \%$

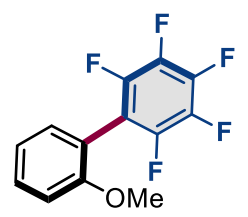

S3b: $57 \%$

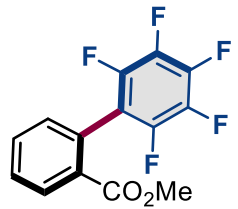

S3c: $6 \%$

\section{Investigation of amine bases}

We found that the use of $\mathrm{Et}_{3} \mathrm{~N}$ as a base improved the yield of the products shown in Table 3. We conducted the solid-state coupling reactions with $\mathrm{Et}_{3} \mathrm{~N}$ at an internal temperature of $80^{\circ} \mathrm{C}$ in order to prevent $\mathrm{Et}_{3} \mathrm{~N}$ (bp: $89^{\circ} \mathrm{C}$ ) from progressing into the gas phase. To clarify the role of such amines, we carried out the reaction of 1 aa with $2 \mathbf{f}$ using $n$ - $\mathrm{Bu}_{3} \mathrm{~N}$ (bp: $217^{\circ} \mathrm{C}$ ) instead of $\mathrm{Et}_{3} \mathrm{~N}$ (bp: $89{ }^{\circ} \mathrm{C}$ ). The reaction provided the coupling product (3ae) in moderate yield (38\% NMR yield). Thus, amines most likely act as bases under these conditions. Unfortunately, we do not have a clear reason why $\mathrm{Et}_{3} \mathrm{~N}$ is particularly effective for these substrates in Table 3 .

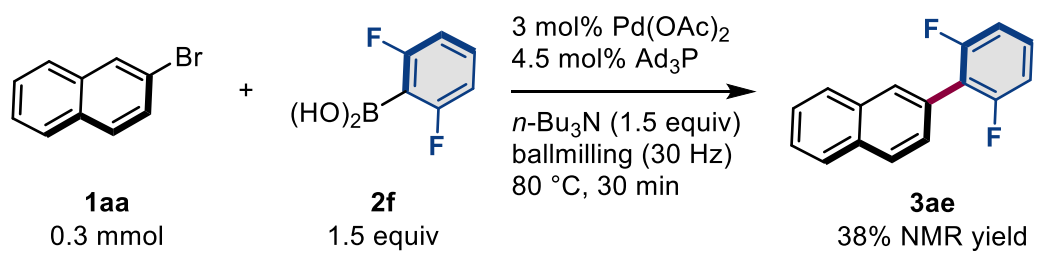




\section{Preliminary NMR Studies}

We have carried out solid-state (SS) NMR measurements to investigate the effect of the solid-state environment on palladium-based catalysts (Figure S6). The SS ${ }^{31} \mathrm{P}$ NMR spectra of the reaction mixtures of 4-bromobiphenyl (1a) and pentafuluorophenyl boronic acid (2a) after ball milling for 10 min showed that the signal derived from SPhos (-7.4 ppm) had completely disappeared, and only one new signal (34.3 ppm) had emerged, suggesting that a SPhos-ligated palladium specie is most likely generated (Figure S6B). Interestingly, the SS ${ }^{31} \mathrm{P}$ NMR spectra of the reaction mixtures of $\mathbf{1 a}$ and $\mathbf{2 a}$ after ball milling for $20 \mathrm{~min}$ or $30 \mathrm{~min}$ showed different signals (20 min: 45.2, 20.7, and -53.4 ppm; 30 min: 42.7, 20.5, and -52.9 ppm; Figure S6C, D). As signals derived from phosphine-ligated palladium species usually appear at relatively high field ( $>0 \mathrm{ppm})$, two kinds of SPhos-ligated palladium species are likely formed after $20 \mathrm{~min}$. On the other hand, the ${ }^{31} \mathrm{P}$ NMR spectra of the reaction mixtures of $\mathbf{1 a}$ and $\mathbf{2 a}$ in 1,4-dioxane- $\mathrm{d}^{8}$ showed multiple complex signals at relatively high field including the oxidized SPhos (44.5-44.6 ppm) that are not observed in the SS ${ }^{31} \mathrm{P}$ NMR spectra (Figure S7). This result suggests that dioxane may coordinate to any off-cycle palladium complexes under solution-based conditions, which would decrease the concentration of coordinatively unsaturated catalytically active species. These preliminary NMR studies thus provide another explanation for the lower reaction efficiency under solution-based conditions compared to that under solid-state conditions.

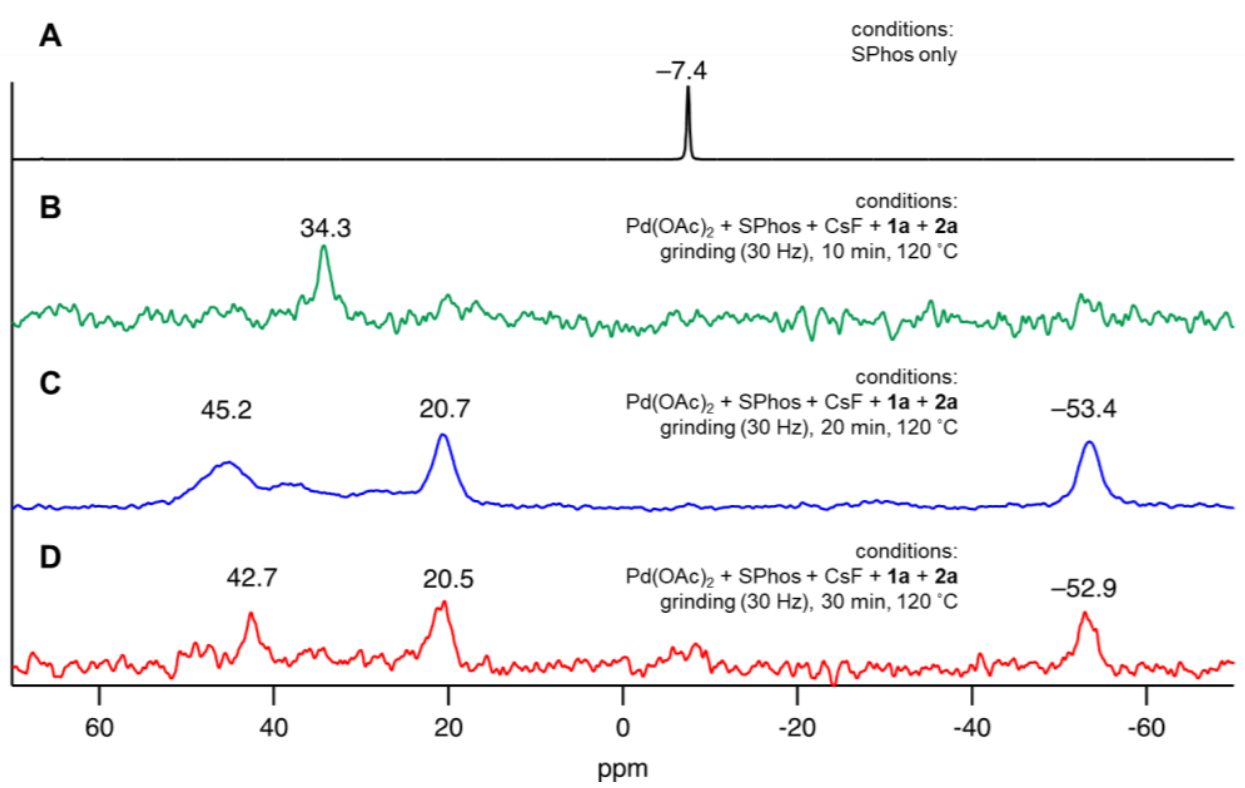

Figure S6. Solid-state ${ }^{31} \mathrm{P}$ NMR spectra of the crude reaction mixture of 1a and 2a under solid-state ball-milling conditions. 


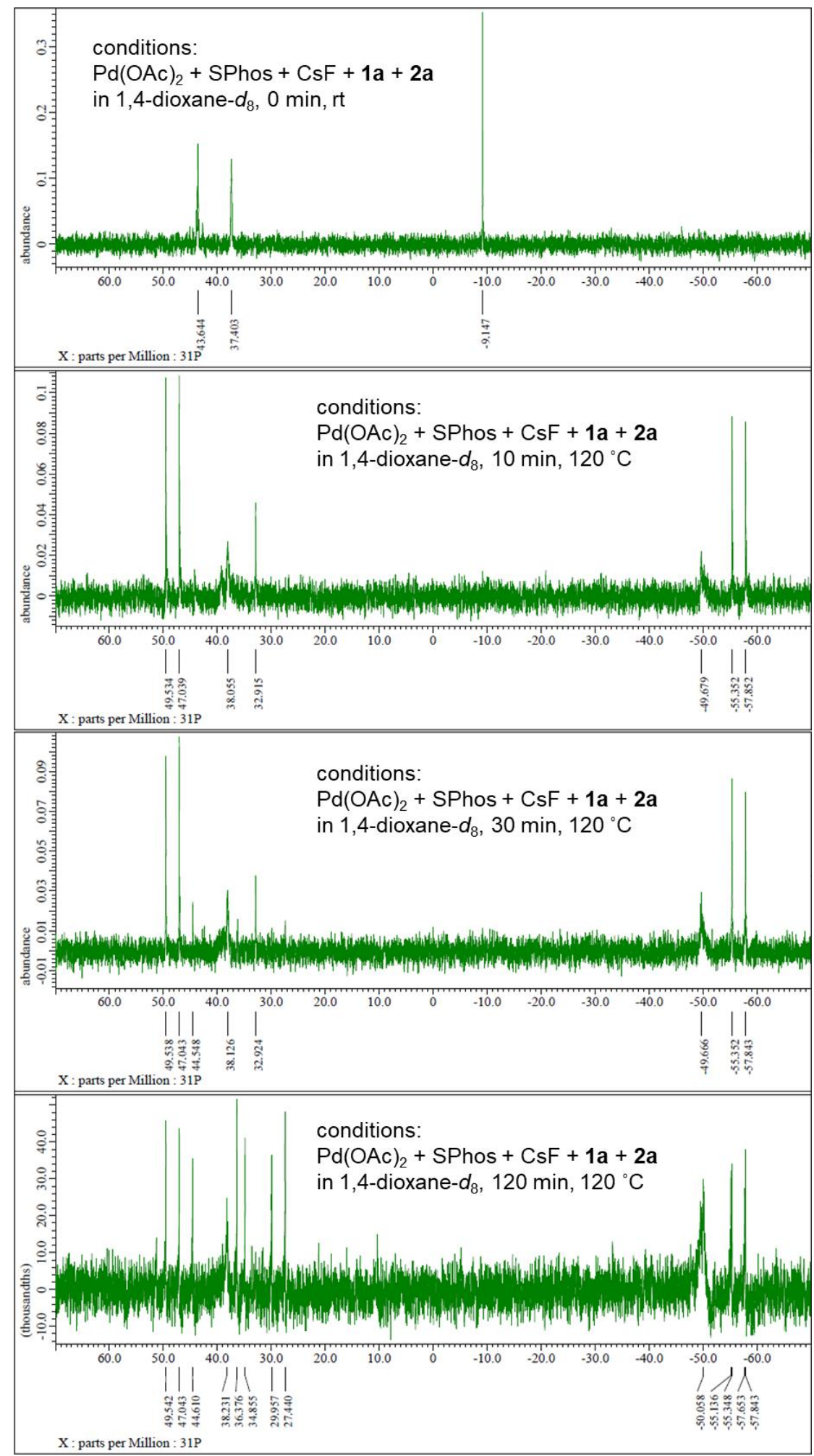

Figure S7. ${ }^{31} \mathrm{P}$ NMR spectra of the crude reaction mixture of 1a and $\mathbf{2 a}$ in 1,4-dioxane-d $\mathrm{d}_{8}$ under the solution-based conditions. 


\section{Additional Mechanistic Studies}

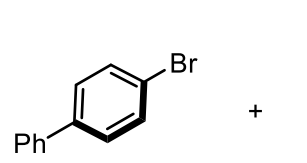

$1 \mathrm{a}$

$0.2 \mathrm{mmol}$

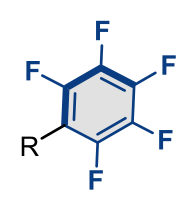

2

1.1equiv
$3 \mathrm{~mol} \% \mathrm{Pd}(\mathrm{OAc})_{2}$

$4.5 \mathrm{~mol} \%$ SPhos

CsF (3.0 equiv) ballmilling $(30 \mathrm{~Hz})$ $120^{\circ} \mathrm{C}, 30 \mathrm{~min}$

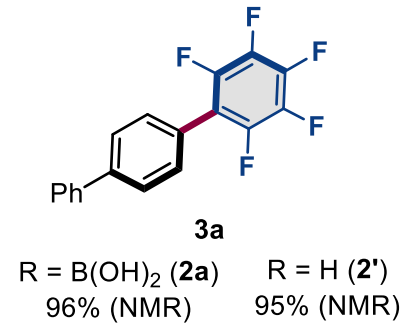<smiles>Brc1ccc(-c2c(-c3ccccc3)c(-c3ccccc3)c(-c3ccccc3)c(-c3ccccc3)c2-c2ccc(Br)cc2)cc1</smiles>

$1 \mathrm{x}$

$0.1 \mathrm{mmol}$
2 (3.0 equiv)

$10 \mathrm{~mol} \% \mathrm{Pd}(\mathrm{OAc})_{2}$

$15 \mathrm{~mol} \%$ SPhos

CsF (6.0 equiv)

$1,5-\operatorname{cod}(0.2 \mu \mathrm{L} / \mathrm{mg})$

ballmilling $(30 \mathrm{~Hz})$

$120^{\circ} \mathrm{C}, 60 \mathrm{~min}$

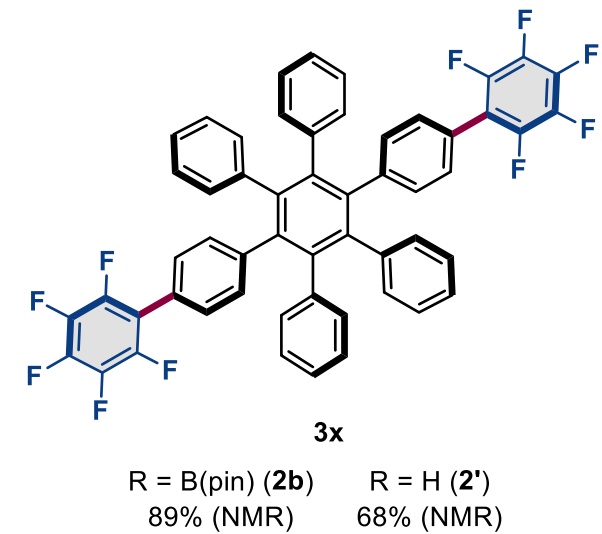

The reaction of pentafluorobenzene (2') with 1a under the optimized solid-state conditions furnished 3a in high yield ( $95 \%$ NMR yield). We also carried out the $\mathrm{C}-\mathrm{H}$ arylation of pentafluorobenzene ( $\mathbf{2}^{\prime}$ ) with $\mathbf{1} \mathbf{x}$ under the optimized solid-state conditions, albeit that the yield of $\mathbf{3 x}$ was decreased (68\%) compared to the reaction of pentafluorophenyl boronic acid (1b) with $\mathbf{1 x}(89 \%)$. Moreover, ${ }^{1} \mathrm{H}$ NMR analysis of the crude reaction mixtures were conducted for all polyfluorinated arylboron reagents used in this study, and these confirmed that no regioisomers and no multiple arylation products were produced. These results suggest that the polyfluoroarylation products should be obtained via the Suzuki-Miyaura coupling as the major pathway, while a protodeboronation/C $-\mathrm{H}$ arylation may proceed simultaneously to form the corresponding products as the minor pathway. 
10. Single Crystal X-ray Structure Analyses

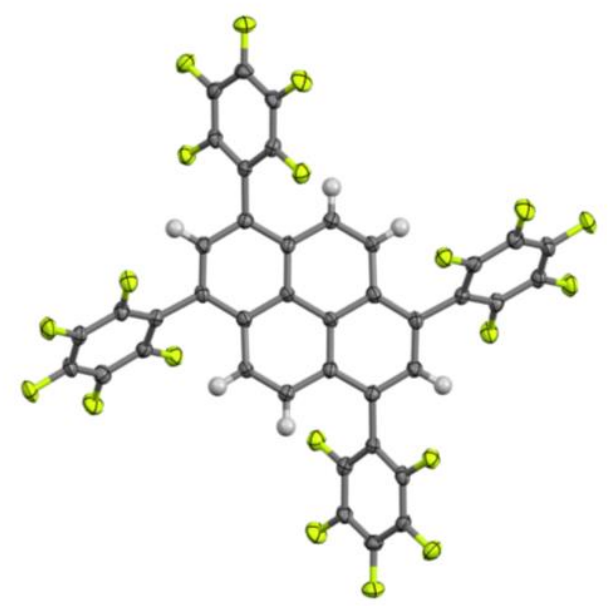

Figure S8. Molecular structure of 3z. Thermal ellipsoids set at 50\% probability. 
Table S4. Summary of X-ray crystallographic data for $\mathbf{3 z}$.

\begin{tabular}{|c|c|}
\hline $\mathrm{CCDC}$ & 2084580 \\
\hline Empirical formula & $\mathrm{C}_{40} \mathrm{H}_{6} \mathrm{~F}_{20}$ \\
\hline Formula weight & 866.45 \\
\hline Temperature/K & 123 \\
\hline Crystal system & triclinic \\
\hline Space group & $P-1$ \\
\hline $\mathrm{a} / \AA \AA$ & $4.3952(1)$ \\
\hline $\mathrm{b} / \AA$ & $18.1094(3)$ \\
\hline $\mathrm{c} / \AA$ & $19.3056(3)$ \\
\hline$\alpha /{ }^{\circ}$ & $82.053(1)$ \\
\hline$\beta /{ }^{\circ}$ & $83.462(2)$ \\
\hline$\gamma /{ }^{\circ}$ & $83.034(2)$ \\
\hline Volume $/ \AA^{3}$ & $1503.24(5)$ \\
\hline $\mathrm{Z}$ & 2 \\
\hline$\rho_{\text {calc }} \mathrm{g} / \mathrm{cm}^{3}$ & 1.914 \\
\hline$\mu / \mathrm{mm}^{-1}$ & 1.804 \\
\hline $\mathrm{F}(000)$ & 852.0 \\
\hline Crystal size $/ \mathrm{mm}^{3}$ & $0.25 \times 0.05 \times 0.05$ \\
\hline Radiation & $\mathrm{CuK} \alpha(\lambda=1.54184)$ \\
\hline $2 \theta$ range for data collection $/^{\circ}$ & 4.644 to 153.028 \\
\hline Index ranges & $-4 \leq \mathrm{h} \leq 5,-22 \leq \mathrm{k} \leq 22,-24 \leq 1 \leq 24$ \\
\hline Reflections collected & 30266 \\
\hline Independent reflections & $5776\left[\mathrm{R}_{\mathrm{int}}=0.0626, \mathrm{R}_{\text {sigma }}=0.0345\right]$ \\
\hline Data/restraints/parameters & $5776 / 0 / 541$ \\
\hline Goodness-of-fit on $\mathrm{F}^{2}$ & 1.111 \\
\hline Final $R$ indexes $[\mathrm{I}>=2 \sigma(\mathrm{I})]$ & $R_{1}=0.0506, w R_{2}=0.1287$ \\
\hline Final $\mathrm{R}$ indexes [all data] & $R_{1}=0.0676, w R_{2}=0.1637$ \\
\hline Largest diff. peak/hole / e $\AA^{-3}$ & $0.38 /-0.41$ \\
\hline
\end{tabular}




\section{Calculation of E-Factors}

We calculated the E-factor of the present solid-state conditions and those of Carrow's conditions according to literature procedures. The E-factor is an index for the quantitative evaluation of the environmental impact of a chemical process. ${ }^{5,6}$

$<$ Our solid-state cross-coupling conditions >
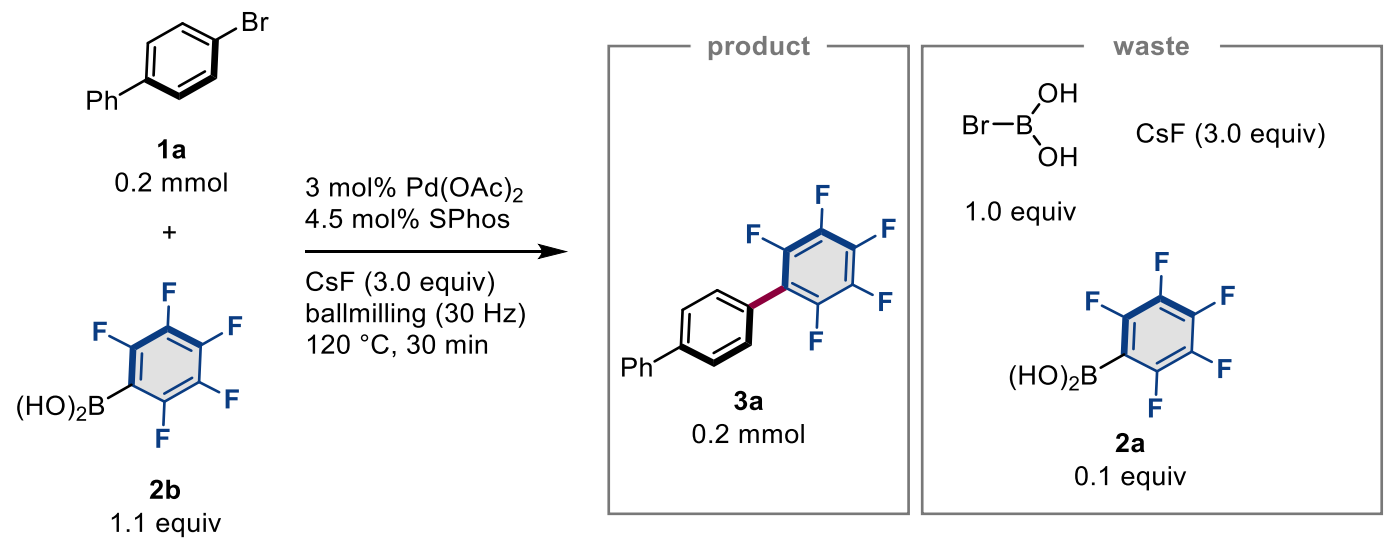

Table S5. E-factor for our solid-state cross-coupling conditions.

\begin{tabular}{cccc}
\hline this work & MW & $\mathrm{mmol}$ & $\mathrm{mg}$ \\
\hline product (3a) & 320.26 & 0.20 & 64.05 \\
\hline waste & $\mathrm{MW}$ & $\mathrm{mmol}$ & $\mathrm{mg}$ \\
\hline $\mathrm{CsF}$ & 151.90 & 0.60 & 91.14 \\
$\mathrm{Br}-\mathrm{B}(\mathrm{OH})_{2}$ & 124.73 & 0.20 & 24.95 \\
2a & 211.88 & 0.020 & 4.24 \\
total & & & 120.32 \\
\hline
\end{tabular}

$\mathbf{E}_{\text {this work }}=120.32 / 64.05=\mathbf{1 . 9}$ 
$<$ Carrow's cross-coupling conditions $>$
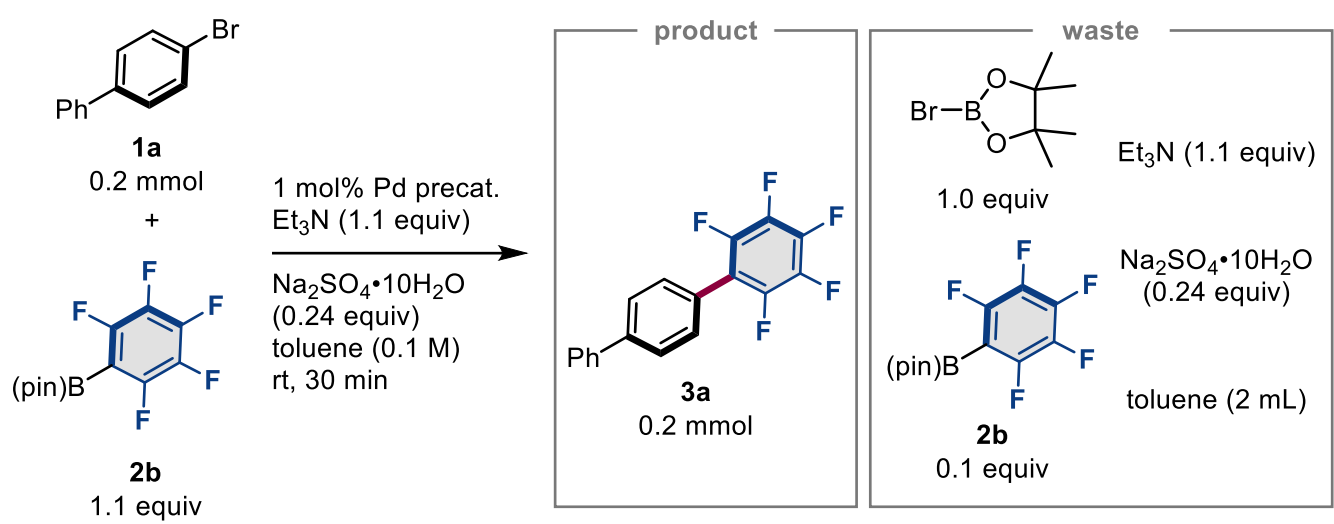

Table S6. E-factor for Carrow's cross-coupling conditions.

\begin{tabular}{cccccc}
\hline Carrow' work & MW & $\mathrm{mmol}$ & $\mathrm{mg}$ & $\mathrm{mL}$ & density \\
\hline product (3a) & 320.26 & 0.20 & 64.05 & & \\
\hline waste & $\mathrm{MW}$ & $\mathrm{mmol}$ & $\mathrm{mg}$ & $\mathrm{mL}$ & density \\
\hline $\mathrm{Et} 3 \mathrm{~N}$ & 101.19 & 0.22 & 22.26 & & \\
$\mathrm{Br}-\mathrm{B}($ pin) & 206.87 & 0.20 & 41.37 & & \\
$\mathrm{Na}_{2} \mathrm{SO}_{4} \cdot 10 \mathrm{H}_{2} \mathrm{O}$ & 322.20 & 0.024 & 7.73 & & \\
toluene & 92.14 & & 1734 & 2.00 & 0.867 \\
2b & 294.03 & 0.020 & 5.88 & & \\
total & & & 1811.25 & & \\
\hline
\end{tabular}

$\mathbf{E}_{\text {Carrow's work }}=1811.25 / 64.05=\mathbf{2 8 . 3}$ 


\section{Characterization of Coupling Products}

\section{2,3,4,5,6-Pentafluoro-1,1':4',1"'-terphenyl (3a).}

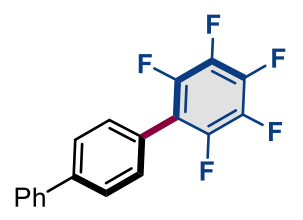

$3 \mathbf{a}$

The reaction was carried out with $46.8 \mathrm{mg}(0.20 \mathrm{mmol})$ of $\mathbf{1 a}$ and $46.8 \mathrm{mg}(0.22 \mathrm{mmol})$ of $\mathbf{2 a}$ according to Procedure A. The product 3a was obtained as a white powder (60.3 mg, $0.19 \mathrm{mmol}, 94 \%$ yield) after purification by silica-gel column chromatography $\left(\mathrm{SiO}_{2}, \mathrm{CH}_{2} \mathrm{Cl}_{2} /\right.$ hexane, 0:100-20:80). ${ }^{1} \mathrm{H}$ and ${ }^{13} \mathrm{C}$ NMR were in agreement with the literature. ${ }^{7}$

${ }^{1} \mathrm{H}$ NMR (396 MHz, $\left.\mathrm{CDCl}_{3}, \delta\right): 7.40(\mathrm{tt}, J=1.5,7.5 \mathrm{~Hz}, 1 \mathrm{H}), 7.46-7.54(\mathrm{~m}, 4 \mathrm{H}), 7.63-7.67(\mathrm{~m}, 2 \mathrm{H})$, $7.72(\mathrm{dt}, J=2.1,8.4 \mathrm{~Hz}, 2 \mathrm{H}) .{ }^{13} \mathrm{C} \mathrm{NMR}\left(99 \mathrm{MHz}, \mathrm{CDCl}_{3}, \delta\right): 115.8\left(\mathrm{td}, J_{C-\mathrm{F}}=3.8,17.5 \mathrm{~Hz}, C\right), 125.4$ (C), $127.2(\mathrm{CH}), 127.4(\mathrm{CH}), 127.9(\mathrm{CH}), 128.9(\mathrm{CH}), 130.6(\mathrm{CH}), 138.0\left(\mathrm{dm}, J_{C-\mathrm{F}}=241.7 \mathrm{~Hz}, C\right)$, $140.3(C), 140.5\left(\mathrm{dm}, J_{C-\mathrm{F}}=260.7 \mathrm{~Hz}, C\right), 144.4\left(\mathrm{dm}, J_{C-\mathrm{F}}=245.5 \mathrm{~Hz}, C\right) .{ }^{19} \mathrm{~F}$ NMR $(369 \mathrm{MHz}$, $\left.\mathrm{CDCl}_{3}, \delta\right):-163.4(\mathrm{~s}, 2 \mathrm{~F}),-156.8-156.7(\mathrm{~m}, 1 \mathrm{~F}),-144.4(\mathrm{~d}, J=21.3 \mathrm{~Hz}, 2 \mathrm{~F})$. HRMS-EI $(\mathrm{m} / \mathrm{z}):[\mathrm{M}]^{+}$ calcd for $\mathrm{C}_{18} \mathrm{H}_{9} \mathrm{~F}_{5}, 320.0624$; found, 320.0622 .

\section{2,3,4,5,6-Pentafluoro-4'-methyl-1,1'-biphenyl (3b).}

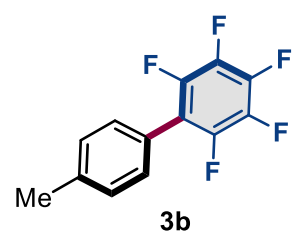

The reaction was carried out with $34.2 \mathrm{mg}(0.20 \mathrm{mmol})$ of $\mathbf{1 b}$ and $64.7 \mathrm{mg}(0.22 \mathrm{mmol})$ of $\mathbf{2} \mathbf{b}$ according to Procedure A. The product $\mathbf{3 b}$ was obtained as a white powder $(46.4 \mathrm{mg}, 0.18 \mathrm{mmol}, 90 \%$ yield) after purification by silica-gel column chromatography $\left(\mathrm{SiO}_{2}\right.$, hexane only). ${ }^{1} \mathrm{H}$ and ${ }^{13} \mathrm{C} \mathrm{NMR}$ were in agreement with the literature. ${ }^{7}$

${ }^{1} \mathrm{H}$ NMR (392 MHz, $\mathrm{CDCl}_{3}, \delta$ ): 2.43 (s, 3H), 7.31 (s, 4H). ${ }^{13} \mathrm{C} \mathrm{NMR} \mathrm{(100} \mathrm{MHz,} \mathrm{CDCl}_{3}, \delta$ ): $21.3\left(\mathrm{CH}_{3}\right)$, $115.9\left(\mathrm{td}, J_{C-\mathrm{F}}=3.8,17.2 \mathrm{~Hz}, C\right), 123.3(C), 129.4(C \mathrm{H}), 129.9(C \mathrm{H}), 137.8\left(\mathrm{dm}, J_{C-\mathrm{F}}=236.9 \mathrm{~Hz}, C\right)$, $139.4(C), 140.2\left(\mathrm{dm}, J_{C-\mathrm{F}}=230.2 \mathrm{~Hz}, C\right), 144.2\left(\mathrm{dm}, J_{C-\mathrm{F}}=238.8 \mathrm{~Hz}, C\right) .{ }^{19} \mathrm{~F} \mathrm{NMR}\left(369 \mathrm{MHz}, \mathrm{CDCl}_{3}\right.$, $\delta):-163.7(\mathrm{td}, J=8.4,21.7 \mathrm{~Hz}, 2 \mathrm{~F}),-157.4(\mathrm{t}, J=20.1 \mathrm{~Hz}, 1 \mathrm{~F}),-144.7(\mathrm{dd}, J=9.2,21.8 \mathrm{~Hz}, 2 \mathrm{~F})$. HRMS-EI $(\mathrm{m} / \mathrm{z})$ : $[\mathrm{M}]^{+}$calcd for $\mathrm{C}_{13} \mathrm{H}_{7} \mathrm{~F}_{5}, 258.0468$; found, 258.0469 . 


\section{2,3,4,5,6-Pentafluoro-3'-methyl-1,1'-biphenyl (3c).}<smiles>COc1cccc(-c2c(F)c(F)c(F)c(F)c2F)c1</smiles>

The reaction was carried out with $34.2 \mathrm{mg}(0.20 \mathrm{mmol})$ of $\mathbf{1 c}$ and $46.6 \mathrm{mg}(0.22 \mathrm{mmol})$ of $\mathbf{2 a}$ according to Procedure A. The product 3c was obtained as a white powder (36.9 $\mathrm{mg}, 0.14 \mathrm{mmol}, 70 \%$ yield) after purification by silica-gel column chromatography $\left(\mathrm{SiO}_{2}\right.$, hexane only). ${ }^{1} \mathrm{H}$ and ${ }^{13} \mathrm{C} \mathrm{NMR}$ were in agreement with the literature. ${ }^{7}$

${ }^{1} \mathrm{H}$ NMR (399 MHz, $\left.\mathrm{CDCl}_{3}, \delta\right): 2.42$ (s, 3H), 7.20-7.29 (m, 3H), 7.38 (t, $\left.J=7.5 \mathrm{~Hz}, 1 \mathrm{H}\right) .{ }^{13} \mathrm{C} \mathrm{NMR}$ $\left(99 \mathrm{MHz}, \mathrm{CDCl}_{3}, \delta\right): 21.4\left(\mathrm{CH}_{3}\right), 116.1\left(\mathrm{td}, J_{C-\mathrm{F}}=3.1,17.7 \mathrm{~Hz}, \mathrm{C}\right), 126.2(\mathrm{C}), 127.2(\mathrm{CH}), 128.6(\mathrm{CH})$, $130.1(\mathrm{CH}), 130.7(\mathrm{CH}), 137.8\left(\mathrm{dm}, J_{C-\mathrm{F}}=253.9 \mathrm{~Hz}, C\right), 138.5(C), 140.3\left(\mathrm{dm}, J_{C-\mathrm{F}}=240.8 \mathrm{~Hz}, C\right)$, $144.1\left(\mathrm{dm}, J_{C-\mathrm{F}}=252.2 \mathrm{~Hz}, C\right) .{ }^{19} \mathrm{~F} \mathrm{NMR}\left(373 \mathrm{MHz}, \mathrm{CDCl}_{3}, \delta\right):-163.7-163.6(\mathrm{~m}, 2 \mathrm{~F}),-157.1(\mathrm{t}, J$ $=22.0 \mathrm{~Hz}, 1 \mathrm{~F}),-144.4(\mathrm{dd}, J=7.8,23.5 \mathrm{~Hz}, 2 \mathrm{~F})$. HRMS-EI $(\mathrm{m} / \mathrm{z})$ : $[\mathrm{M}]^{+}$calcd for $\mathrm{C}_{13} \mathrm{H}_{7} \mathrm{~F}_{5}, 258.0468$; found, 258.0464 .

\section{2,3,4,5,6-Pentafluoro-4'-methoxy-1,1'-biphenyl (3e).}

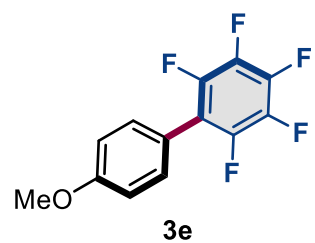

The reaction was carried out with $37.1 \mathrm{mg}(0.20 \mathrm{mmol})$ of $\mathbf{1 d}$ and $65.4 \mathrm{mg}(0.22 \mathrm{mmol})$ of $\mathbf{2} \mathbf{b}$ according to Procedure A. The product 3e was obtained as a white powder (49.7 mg, $0.18 \mathrm{mmol}, 91 \%$ yield) after purification by silica-gel column chromatography $\left(\mathrm{SiO}_{2}\right.$, EtOAc/hexane, 0:100-8:92). ${ }^{1} \mathrm{H}$ and ${ }^{13} \mathrm{C}$ NMR were in agreement with the literature. ${ }^{8}$

${ }^{1} \mathrm{H} \mathrm{NMR}\left(396 \mathrm{MHz}, \mathrm{CDCl}_{3}, \delta\right): 3.87$ (s, 3H), 7.02 (dt, $\left.J=2.5,9.5 \mathrm{~Hz}, 2 \mathrm{H}\right), 7.34-7.39(\mathrm{~m}, 2 \mathrm{H}) .{ }^{13} \mathrm{C}$ NMR (100 MHz, $\left.\mathrm{CDCl}_{3}, \delta\right): 55.3\left(\mathrm{CH}_{3}\right), 114.2(\mathrm{CH}), 115.7\left(\mathrm{td}, J_{C-\mathrm{F}}=3.8,17.2 \mathrm{~Hz}, C\right), 118.3(C)$, $131.4(C \mathrm{H}), 137.8\left(\mathrm{dm}, J_{C-\mathrm{F}}=251.9 \mathrm{~Hz}, C\right), 140.2\left(\mathrm{dm}, J_{C-\mathrm{F}}=219.4 \mathrm{~Hz}, C\right), 144.1\left(\mathrm{dm}, J_{C-\mathrm{F}}=243.4\right.$ $\mathrm{Hz}, C), 160.2(C) .{ }^{19} \mathrm{~F}$ NMR (373 MHz, $\left.\mathrm{CDCl}_{3}, \delta\right):-163.8$ (t, $\left.J=22.8 \mathrm{~Hz}, 2 \mathrm{~F}\right),-157.8$ (t, $J=20.0 \mathrm{~Hz}$, $1 \mathrm{~F}),-144.9$ (dd, $J=11.6,22.8 \mathrm{~Hz}, 2 \mathrm{~F})$. HRMS-EI $(\mathrm{m} / \mathrm{z})$ : $[\mathrm{M}]^{+}$calcd for $\mathrm{C}_{13} \mathrm{H}_{7} \mathrm{~F}_{5}, 274.0417$; found, 274.0413. 


\section{2,3,4,4',5,6-Hexafluoro-1,1'-biphenyl (3f).}

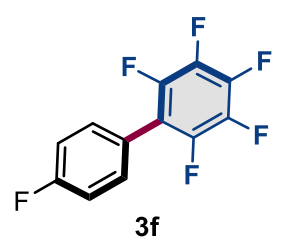

The reaction was carried out with $33.5 \mathrm{mg}(0.20 \mathrm{mmol})$ of $\mathbf{1 f}$ and $47.4 \mathrm{mg}(0.22 \mathrm{mmol})$ of $\mathbf{2 a}$ according to Procedure A. The product $3 \mathbf{f}$ was obtained as a white powder ( $42.9 \mathrm{mg}, 0.16 \mathrm{mmol}, 85 \%$ yield) after purification by silica-gel column chromatography $\left(\mathrm{SiO}_{2}\right.$, hexane only). ${ }^{1} \mathrm{H}$ and ${ }^{13} \mathrm{C}$ NMR were in agreement with the literature. ${ }^{7}$

${ }^{1} \mathrm{H}$ NMR (396 MHz, $\mathrm{CDCl}_{3}, \delta$ ): 7.17-7.23 (m, 2H), 7.39-7.44 (m, 2H). ${ }^{13} \mathrm{C} \mathrm{NMR} \mathrm{(100} \mathrm{MHz,} \mathrm{CDCl}_{3}$,

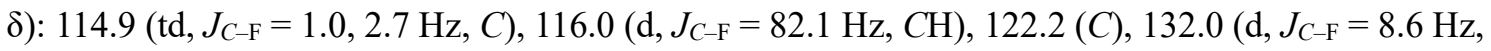
C), $137.8\left(\mathrm{dm}, J_{C-\mathrm{F}}=253.4 \mathrm{~Hz}, C\right), 140.4\left(\mathrm{dm}, J_{C-\mathrm{F}}=254.8 \mathrm{~Hz}, C\right), 144.1\left(\mathrm{dm}, J_{C-\mathrm{F}}=244.3 \mathrm{~Hz}, C\right)$, $163.1\left(\mathrm{dm}, J_{C-\mathrm{F}}=251.0 \mathrm{~Hz}, C\right) .{ }^{19} \mathrm{~F} \mathrm{NMR}\left(373 \mathrm{MHz}, \mathrm{CDCl}_{3}, \delta\right):-163.2(\mathrm{td}, J=8.3,21.8 \mathrm{~Hz}, 2 \mathrm{~F}),-$ $156.4(\mathrm{t}, J=20.3 \mathrm{~Hz}, 1 \mathrm{~F}),-144.6(\mathrm{dd}, J=7.8,23.5 \mathrm{~Hz}, 2 \mathrm{~F}),-112.6-112.5(\mathrm{~m}, 1 \mathrm{~F})$. HRMS-EI $(\mathrm{m} / \mathrm{z})$ : $[\mathrm{M}]^{+}$calcd for $\mathrm{C}_{12} \mathrm{H}_{4} \mathrm{~F}_{6}, 262.0217$; found, 262.0218 .

\section{2,3,4,5,6-Pentafluoro-4'-(trifluoromethyl)-1,1'-biphenyl (3g).}<smiles>Fc1c(F)c(F)c(-c2ccc(C(F)(F)F)cc2)c(F)c1F</smiles>

The reaction was carried out with $44.3 \mathrm{mg}(0.20 \mathrm{mmol})$ of $1 \mathrm{~g}$ and $46.6 \mathrm{mg}(0.22 \mathrm{mmol})$ of $\mathbf{2 a}$ according to Procedure A. The product 3g was obtained as a white powder (32.3 $\mathrm{mg}, 0.10 \mathrm{mmol}, 53 \%$ yield) after purification by silica-gel column chromatography $\left(\mathrm{SiO}_{2}\right.$, hexane only). ${ }^{1} \mathrm{H}$ and ${ }^{13} \mathrm{C}$ NMR were in agreement with the literature. ${ }^{7}$

${ }^{1} \mathrm{H}$ NMR $\left(396 \mathrm{MHz}, \mathrm{CDCl}_{3}, \delta\right): 7.57$ (d, $\left.J=7.9 \mathrm{~Hz}, 2 \mathrm{H}\right), 7.77$ (d, $\left.J=7.9 \mathrm{~Hz}, 2 \mathrm{H}\right) .{ }^{13} \mathrm{C}$ NMR $(99 \mathrm{MHz}$, $\mathrm{CDCl}_{3}, \delta$ ): $114.5\left(\mathrm{td}, J_{C-\mathrm{F}}=4.1,17.0 \mathrm{~Hz}, C\right), 123.8$ (q, $\left.J_{C-\mathrm{F}}=273.8 \mathrm{~Hz}, C\right), 125.7$ (q, $J_{C-\mathrm{F}}=3.5 \mathrm{~Hz}$, $C \mathrm{H}), 130.1(C), 130.6(C \mathrm{H}), 131.4\left(\mathrm{q}, J_{C-\mathrm{F}}=33.1 \mathrm{~Hz}, \mathrm{C}\right), 137.8$ (dm, $\left.J_{C-\mathrm{F}}=238.0 \mathrm{~Hz}, C\right), 140.7$ (dm, $\left.J_{C-\mathrm{F}}=299.4 \mathrm{~Hz}, C\right), 144.2\left(\mathrm{dm}, J_{C-\mathrm{F}}=243.6 \mathrm{~Hz}, C\right) .{ }^{19} \mathrm{~F} \mathrm{NMR}\left(369 \mathrm{MHz}, \mathrm{CDCl}_{3}, \delta\right):-162.6(\mathrm{td}, J=$ 6.3, $22.0 \mathrm{~Hz}, 2 \mathrm{~F}),-155.0(\mathrm{t}, J=20.3 \mathrm{~Hz}, 1 \mathrm{~F}),-144.2(\mathrm{dd}, J=7.8,23.5 \mathrm{~Hz}, 2 \mathrm{~F}),-64.2$ (s, 3F). HRMS$\mathrm{EI}(\mathrm{m} / \mathrm{z})$ : $[\mathrm{M}]^{+}$calcd for $\mathrm{C}_{13} \mathrm{H}_{4} \mathrm{~F}_{8}, 312.0185$; found, 312.0182 . 
Methyl 2',3',4',5',6'-pentafluoro-(1,1'-biphenyl)-4-carboxylate (3h).<smiles>COC(=O)c1ccc(-c2c(F)c(F)c(F)c(F)c2F)cc1</smiles>

The reaction was carried out with $42.8 \mathrm{mg}(0.20 \mathrm{mmol})$ of $\mathbf{1 h}$ and $65.7 \mathrm{mg}(0.22 \mathrm{mmol})$ of $\mathbf{2 b}$ according to Procedure A. The product $\mathbf{3 h}$ was obtained as a white powder (57.9 mg, $0.19 \mathrm{mmol}, 96 \%$ yield) after purification by silica-gel column chromatography $\left(\mathrm{SiO}_{2}\right.$, EtOAc/hexane, 0:100-4:96). ${ }^{1} \mathrm{H}$ and ${ }^{13} \mathrm{C}$ NMR were in agreement with the literature. ${ }^{7}$

${ }^{1} \mathrm{H}$ NMR $\left(396 \mathrm{MHz}, \mathrm{CDCl}_{3}, \delta\right): 3.96$ (s, 3H), $7.52(\mathrm{~d}, J=8.7 \mathrm{~Hz}, 2 \mathrm{H}), 8.17$ (d, $\left.J=8.3 \mathrm{~Hz}, 2 \mathrm{H}\right) .{ }^{13} \mathrm{C}$ NMR (100 MHz, $\left.\mathrm{CDCl}_{3}, \delta\right): 52.3\left(\mathrm{CH}_{3}\right), 114.9\left(\mathrm{td}, J_{C-\mathrm{F}}=3.5,16.8 \mathrm{~Hz}, C\right), 129.8(\mathrm{CH}), 130.2(\mathrm{CH})$, $130.9(C), 137.9\left(\mathrm{dm}, J_{C-\mathrm{F}}=254.8 \mathrm{~Hz}, C\right), 140.8\left(\mathrm{dm}, J_{C-\mathrm{F}}=251.0 \mathrm{~Hz}, C\right), 144.1\left(\mathrm{dm}, J_{C-\mathrm{F}}=239.5\right.$ $\mathrm{Hz}, C), 166.3(C) .{ }^{19} \mathrm{~F} \mathrm{NMR}\left(373 \mathrm{MHz}, \mathrm{CDCl}_{3}, \delta\right):-162.8$ (t, $\left.J=22.8 \mathrm{~Hz}, 2 \mathrm{~F}\right),-155.4(\mathrm{t}, J=20.0 \mathrm{~Hz}$, $1 \mathrm{~F}),-144.1(\mathrm{~d}, J=22.8 \mathrm{~Hz}, 2 \mathrm{~F})$. HRMS-EI $(\mathrm{m} / \mathrm{z})$ : $[\mathrm{M}]^{+}$calcd for $\mathrm{C}_{14} \mathrm{H}_{7} \mathrm{~F}_{5} \mathrm{O}_{2}, 302.0366$; found, 302.0361 .

$2^{\prime}, 3^{\prime}, 4^{\prime}, 5^{\prime}, 6^{\prime}$-Pentafluoro-(1,1'-biphenyl)-4-carbonitrile (3i).

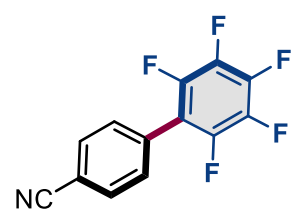

$3 \mathbf{i}$

The reaction was carried out with $36.4 \mathrm{mg}(0.20 \mathrm{mmol})$ of $1 \mathbf{i}$ and $63.4 \mathrm{mg}(0.30 \mathrm{mmol})$ of $2 \mathbf{2}$ according to Procedure A. The product 3i was obtained as a white powder (39.2 $\mathrm{mg}, 0.15 \mathrm{mmol}, 73 \%$ yield) after purification by silica-gel column chromatography $\left(\mathrm{SiO}_{2}\right.$, EtOAc/hexane, 0:100-6:94). ${ }^{1} \mathrm{H}$ and ${ }^{13} \mathrm{C}$ NMR were in agreement with the literature. ${ }^{7}$

${ }^{1} \mathrm{H}$ NMR $\left(396 \mathrm{MHz}, \mathrm{CDCl}_{3}, \delta\right): 7.57$ (d, $\left.J=8.3 \mathrm{~Hz}, 2 \mathrm{H}\right), 7.80-7.82(\mathrm{~m}, 2 \mathrm{H}) .{ }^{13} \mathrm{C} \mathrm{NMR}(99 \mathrm{MHz}$, $\left.\mathrm{CDCl}_{3}, \delta\right): 113.3(C), 114.0\left(\mathrm{td}, J_{C-\mathrm{F}}=3.8,16.7 \mathrm{~Hz}, C\right), 118.1(C), 130.9(\mathrm{CH}), 131.1(C), 132.4(\mathrm{CH})$, $137.9\left(\mathrm{dm}, J_{C-\mathrm{F}}=257.5 \mathrm{~Hz}, C\right), 141.1\left(\mathrm{dm}, J_{C-\mathrm{F}}=259.4 \mathrm{~Hz}, C\right), 144.0\left(\mathrm{dm}, J_{C-\mathrm{F}}=257.6 \mathrm{~Hz}, C\right) .{ }^{19} \mathrm{~F}$ $\operatorname{NMR}\left(373 \mathrm{MHz}, \mathrm{CDCl}_{3}, \delta\right):-162.2-162.1$ (m, 2F), -154.0 (t, $\left.J=20.3 \mathrm{~Hz}, 1 \mathrm{~F}\right),-144.0$ (dd, $J=9.3$, $21.6 \mathrm{~Hz}, 2 \mathrm{~F})$. HRMS-EI $(\mathrm{m} / \mathrm{z})$ : [M] $]^{+}$calcd for $\mathrm{C}_{13} \mathrm{H}_{4} \mathrm{~F}_{5} \mathrm{~N}_{1}, 269.0264$; found, 269.0272 . 


\section{2-Methoxy-6-(perfluorophenyl)pyridine (3j).}<smiles>COc1cccc(-c2c(F)c(F)c(F)c(F)c2F)n1</smiles>

The reaction was carried out with $36.9 \mathrm{mg}(0.20 \mathrm{mmol})$ of $\mathbf{1} \mathbf{j}$ and $64.6 \mathrm{mg}(0.22 \mathrm{mmol})$ of $\mathbf{2} \mathbf{b}$ according to Procedure A. The product $\mathbf{3 j}$ was obtained as a colorless oil ( $42.3 \mathrm{mg}, 0.15 \mathrm{mmol}, 78 \%$ yield) after purification by silica-gel column chromatography $\left(\mathrm{SiO}_{2}\right.$, EtOAc/hexane, 0:100-8:92). ${ }^{1} \mathrm{H}$ and ${ }^{13} \mathrm{C}$ NMR were in agreement with the literature. ${ }^{9}$

${ }^{1} \mathrm{H}$ NMR (396 MHz, $\left.\mathrm{CDCl}_{3}, \delta\right): 3.93$ (s, 3H), 6.83 (d, $\left.J=7.9 \mathrm{~Hz}, 1 \mathrm{H}\right), 7.05$ (d, $\left.J=7.1 \mathrm{~Hz}, 1 \mathrm{H}\right), 7.69$ $(\mathrm{dd}, J=7.3,8.5 \mathrm{~Hz}, 1 \mathrm{H}) \cdot{ }^{13} \mathrm{C} \mathrm{NMR}\left(100 \mathrm{MHz}, \mathrm{CDCl}_{3}, \delta\right): 53.6\left(\mathrm{CH}_{3}\right), 111.4(\mathrm{CH}), 115.3\left(\mathrm{td}, J_{C-\mathrm{F}}=\right.$ 3.8, 16.3 Hz, C), $118.8(C \mathrm{H}), 137.8\left(\mathrm{dm}, J_{C-\mathrm{F}}=271.1 \mathrm{~Hz}, C\right), 138.9(\mathrm{CH}), 141.0\left(\mathrm{dm}, J_{C-\mathrm{F}}=255.8 \mathrm{~Hz}\right.$, C), $143.8(C), 144.7\left(\mathrm{dm}, J_{C-\mathrm{F}}=251.9 \mathrm{~Hz}, C\right), 163.9(C) .{ }^{19} \mathrm{~F}$ NMR (373 MHz, $\left.\mathrm{CDCl}_{3}, \delta\right):-163.5$ (t, $J$ $=22.9 \mathrm{~Hz}, 2 \mathrm{~F}),-155.9(\mathrm{~d}, J=22.8 \mathrm{~Hz}, 1 \mathrm{~F}),-144.0(\mathrm{~d}, J=22.8 \mathrm{~Hz}, 2 \mathrm{~F})$. HRMS-API $(m / z):[\mathrm{M}+\mathrm{H}]^{+}$ calcd for $\mathrm{C}_{12} \mathrm{H}_{7} \mathrm{~F}_{5} \mathrm{~N}_{1} \mathrm{O}_{1}, 275.0370$; found, 276.0441 .

2',3',4',5',6'-Pentafluoro- $N, N$-diphenyl-(1,1'-biphenyl)-4-amine (3k).

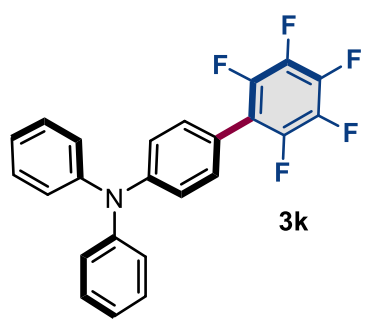

The reaction was carried out with $64.5 \mathrm{mg}(0.20 \mathrm{mmol})$ of $1 \mathbf{k}$ and $63.7 \mathrm{mg}(0.30 \mathrm{mmol})$ of $\mathbf{2 a}$ according to Procedure A. The product 3k was obtained as a colorless oil (75.8 $\mathrm{mg}, 0.18 \mathrm{mmol}, 93 \%$ yield) after purification by silica-gel column chromatography $\left(\mathrm{SiO}_{2}, \mathrm{CH}_{2} \mathrm{Cl}_{2} /\right.$ hexane, 0:100-5:95).

${ }^{1} \mathrm{H}$ NMR (396 MHz, $\left.\mathrm{CDCl}_{3}, \delta\right): 7.05-7.14(\mathrm{~m}, 4 \mathrm{H}), 7.16$ (d. $\left.J=7.1 \mathrm{~Hz}, 4 \mathrm{H}\right), 7.25-7.33(\mathrm{~m}, 6 \mathrm{H}) .{ }^{13} \mathrm{C}$ NMR (99 MHz, $\left.\mathrm{CDCl}_{3}, \delta\right): 115.7\left(\mathrm{td}, J_{C-\mathrm{F}}=3.5,16.8 \mathrm{~Hz}, C\right), 118.8(C), 121.9(\mathrm{CH}), 123.7(\mathrm{CH})$, $125.2(\mathrm{CH}), 129.4(\mathrm{CH}), 130.9(\mathrm{CH}), 137.9\left(\mathrm{dm}, J_{C-\mathrm{F}}=236.1 \mathrm{~Hz}, C\right), 139.9\left(\mathrm{dm}, J_{C-\mathrm{F}}=241.8 \mathrm{~Hz}, C\right)$, $144.1\left(\mathrm{dm}, J_{C-\mathrm{F}}=254.4 \mathrm{~Hz}, C\right), 147.1(C), 148.7(C) .{ }^{19} \mathrm{~F} \mathrm{NMR}\left(369 \mathrm{MHz}, \mathrm{CDCl}_{3}, \delta\right):-163.8(\mathrm{td}, J=$ $7.3,22.5 \mathrm{~Hz}, 2 \mathrm{~F}),-157.9$ (t, $J=21.8 \mathrm{~Hz}, 1 \mathrm{~F}),-144.8(\mathrm{dd}, J=7.7,23.2 \mathrm{~Hz}, 2 \mathrm{~F})$. HRMS-EI $(\mathrm{m} / \mathrm{z}):[\mathrm{M}]^{+}$ calcd for $\mathrm{C}_{24} \mathrm{H}_{14} \mathrm{~F}_{5} \mathrm{~N}_{1}, 411.1046$; found, 411.1043 . 
1-(Perfluorophenyl)-4-phenylnaphthalene (3I).

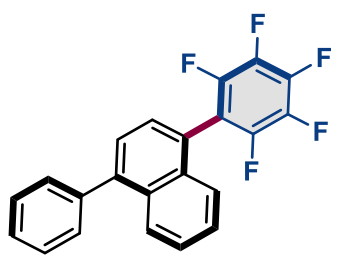

3)

The reaction was carried out with $56.8 \mathrm{mg}(0.20 \mathrm{mmol})$ of $\mathbf{1 l}$ and $65.1 \mathrm{mg}(0.22 \mathrm{mmol})$ of $\mathbf{2 b}$ according to Procedure A. The product $\mathbf{3 1}$ was obtained as a white powder (59.1 $\mathrm{mg}, 0.16 \mathrm{mmol}, 80 \%$ yield) after purification by silica-gel column chromatography $\left(\mathrm{SiO}_{2}, \mathrm{CH}_{2} \mathrm{Cl}_{2} /\right.$ hexane, 0:100-10:90).

${ }^{1} \mathrm{H}$ NMR (396 MHz, $\mathrm{CDCl}_{3}, \delta$ ): 7.45-7.57 (m, 10H), 7.97-8.02 (m, 1H). ${ }^{13} \mathrm{C}$ NMR (100 MHz, $\mathrm{CDCl}_{3}$,

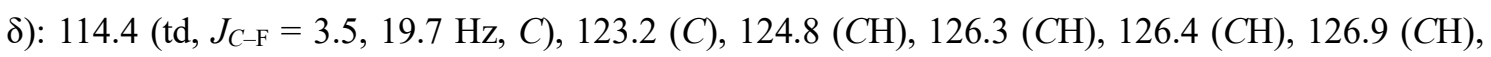
127.6 (CH), $128.4(\mathrm{CH}), 128.5(\mathrm{CH}), 130.0(\mathrm{CH}), 131.8(C), 132.0(C), 137.8\left(\mathrm{dm}, J_{C-\mathrm{F}}=253.9 \mathrm{~Hz}\right.$, C), $140.1(C), 140.9\left(\mathrm{dm}, J_{C-\mathrm{F}}=254.9 \mathrm{~Hz}, C\right), 142.4(C), 144.7\left(\mathrm{dm}, J_{C-\mathrm{F}}=251.9 \mathrm{~Hz}, C\right) .{ }^{19} \mathrm{~F} \mathrm{NMR}$ (373 MHz, $\left.\mathrm{CDCl}_{3}, \delta\right):-163.0$ (t, $\left.J=22.8 \mathrm{~Hz}, 2 \mathrm{~F}\right),-155.8$ (t, $\left.J=22.8 \mathrm{~Hz}, 1 \mathrm{~F}\right),-140.6$ (d, $J=22.8 \mathrm{~Hz}$, 2F). HRMS-EI ( $\mathrm{m} / \mathrm{z})$ : $[\mathrm{M}]^{+}$calcd for $\mathrm{C}_{22} \mathrm{H}_{11} \mathrm{~F}_{5}, 370.0781$; found, 370.0777 .

\section{9-(Perfluorophenyl)anthracene (3m).}

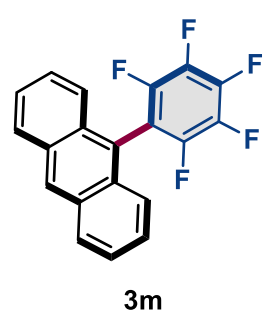

The reaction was carried out with $51.5 \mathrm{mg}(0.20 \mathrm{mmol})$ of $\mathbf{1 m}$ and $88.8 \mathrm{mg}(0.30 \mathrm{mmol})$ of $\mathbf{2 b}$ according to Procedure A. The product 3m was obtained as a yellow powder $(67.5 \mathrm{mg}, 0.20 \mathrm{mmol}$, $98 \%$ yield) after purification by silica-gel column chromatography $\left(\mathrm{SiO}_{2}, \mathrm{CH}_{2} \mathrm{Cl}_{2} /\right.$ hexane, 0:100-10:90).

${ }^{1} \mathrm{H}$ NMR (396 MHz, $\left.\mathrm{CDCl}_{3}, \delta\right): 7.46-7.55$ (m, 6H), 8.08-8.14 (m, 2H), 8.64 (s, 1H). ${ }^{13} \mathrm{C}$ NMR (99 $\left.\mathrm{MHz}, \mathrm{CDCl}_{3}, \delta\right): 112.5\left(\mathrm{td}, J_{C-\mathrm{F}}=2.5,20.5 \mathrm{~Hz}, C\right), 119.6(C), 124.6(\mathrm{CH}), 125.5(\mathrm{CH}), 127.0(\mathrm{CH})$, $128.9(C \mathrm{H}), 129.5(\mathrm{CH}), 130.6(C), 131.2(C), 137.9\left(\mathrm{dm}, J_{C-\mathrm{F}}=254.9 \mathrm{~Hz}, C\right), 141.3\left(\mathrm{dm}, J_{C-\mathrm{F}}=255.9\right.$ $\mathrm{Hz}, C), 144.9\left(\mathrm{dm}, J_{C-\mathrm{F}}=249.3 \mathrm{~Hz}, C\right) .{ }^{19} \mathrm{~F} \mathrm{NMR}\left(373 \mathrm{MHz}, \mathrm{CDCl}_{3}, \delta\right):-162.7-162.6(\mathrm{~m}, 2 \mathrm{~F}),-$ $154.9(\mathrm{t}, J=6.1 \mathrm{~Hz}, 1 \mathrm{~F}),-139.3(\mathrm{~d}, J=4.6 \mathrm{~Hz}, 2 \mathrm{~F})$. HRMS-EI $(\mathrm{m} / \mathrm{z}):[\mathrm{M}]^{+}$calcd for $\mathrm{C}_{20} \mathrm{H}_{9} \mathrm{~F}_{5}$, 344.0624; found, 344.0627 . 


\section{9-(Perfluorophenyl)phenanthrene (3n).}

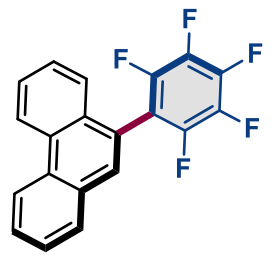

$3 n$

The reaction was carried out with $51.3 \mathrm{mg}(0.20 \mathrm{mmol})$ of $\mathbf{1 n}$ and $65.4 \mathrm{mg}(0.22 \mathrm{mmol})$ of $\mathbf{2 b}$ according to Procedure A. The product 3n was obtained as a white powder (65.3 mg, $0.19 \mathrm{mmol}, 95 \%$ yield) after purification by silica-gel column chromatography $\left(\mathrm{SiO}_{2}\right.$, hexane only).

${ }^{1} \mathrm{H} \mathrm{NMR}\left(392 \mathrm{MHz}, \mathrm{CDCl}_{3}, \delta\right): 7.50-7.54(\mathrm{~m}, 1 \mathrm{H}), 7.60$ (td, $\left.J=0.8,7.6 \mathrm{~Hz}, 1 \mathrm{H}\right), 7.64(\mathrm{td}, J=1.0$, $7.4 \mathrm{~Hz}, 1 \mathrm{H}), 7.70-7.79(\mathrm{~m}, 3 \mathrm{H}), 7.93(\mathrm{~d}, J=8.6 \mathrm{~Hz}, 1 \mathrm{H}), 8.78(\mathrm{dd}, J=8.2,17.2 \mathrm{~Hz}, 2 \mathrm{H}) .{ }^{13} \mathrm{C} \mathrm{NMR}$ $\left(100 \mathrm{MHz}, \mathrm{CDCl}_{3}, \delta\right): 114.4\left(\mathrm{td}, J_{C-\mathrm{F}}=3.5,19.6 \mathrm{~Hz}, C\right), 122.6(\mathrm{C}), 122.7(\mathrm{CH}), 123.2(\mathrm{CH}), 125.3$ $(\mathrm{CH}), 127.1(\mathrm{CH}), 127.2(\mathrm{CH}), 127.9(\mathrm{CH}), 129.0(\mathrm{CH}), 130.0(\mathrm{C}), 130.4(\mathrm{CH}), 130.6(\mathrm{C}), 130.8(\mathrm{C})$, $137.7\left(\mathrm{dm}, J_{C-\mathrm{F}}=254.8 \mathrm{~Hz}, C\right), 141.0\left(\mathrm{dm}, J_{C-\mathrm{F}}=255.8 \mathrm{~Hz}, C\right), 144.8\left(\mathrm{dm}, J_{C-\mathrm{F}}=249.1 \mathrm{~Hz}, C\right) .{ }^{19} \mathrm{~F}$ NMR (369 MHz, $\left.\mathrm{CDCl}_{3}, \delta\right):-163.0-162.9(\mathrm{~m}, 2 \mathrm{~F}),-155.8-155.6(\mathrm{~m}, 1 \mathrm{~F}),-140.4$ (dd, $J=11.4$, $22.9 \mathrm{~Hz}, 2 \mathrm{~F})$. HRMS-EI $(\mathrm{m} / \mathrm{z})$ : [M] ${ }^{+}$calcd for $\mathrm{C}_{20} \mathrm{H}_{9} \mathrm{~F}_{5}, 3344.0624$; found, 344.0630 .

\section{1-(Perfluorophenyl)pyrene (3o).}

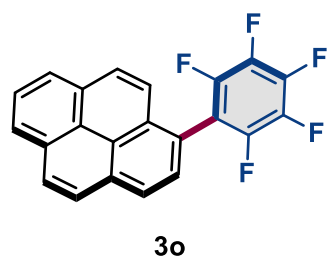

The reaction was carried out with $56.6 \mathrm{mg}(0.20 \mathrm{mmol})$ of $\mathbf{1 o}$ and $64.7 \mathrm{mg}(0.22 \mathrm{mmol})$ of $\mathbf{2} \mathbf{b}$ according to Procedure A. The product 30 was obtained as a white powder (53.2 mg, $0.14 \mathrm{mmol}, 73 \%$ yield) after purification by silica-gel column chromatography $\left(\mathrm{SiO}_{2}\right.$, hexane only).

${ }^{1} \mathrm{H}$ NMR (392 MHz, $\mathrm{CDCl}_{3}, \delta$ ): 7.74 (dt, $\left.J=1.9,9.1 \mathrm{~Hz}, 1 \mathrm{H}\right), 7.94$ (d, $\left.J=7.8 \mathrm{~Hz}, 1 \mathrm{H}\right), 8.05-8.21$ (m, $4 \mathrm{H}), 8.23-8.32(\mathrm{~m}, 3 \mathrm{H}) .{ }^{13} \mathrm{C} \mathrm{NMR}\left(151 \mathrm{MHz}, \mathrm{CDCl}_{3}, \delta\right): 114.9$ (td, $\left.J_{C-\mathrm{F}}=3.9,18.8 \mathrm{~Hz}, C\right), 120.5(C)$, $123.7(\mathrm{CH}), 124.4(\mathrm{C}), 124.6(\mathrm{CH}), 124.8(\mathrm{C}), 125.7(\mathrm{CH}), 126.0(\mathrm{CH}), 126.3(\mathrm{CH}), 127.1(\mathrm{CH}), 128.2$ $(C H), 128.6(C H), 128.8(C H), 129.7(C), 130.6(C), 131.1(C), 132.2(C), 137.8\left(\mathrm{dm}, J_{C-\mathrm{F}}=251.6\right.$ $\mathrm{Hz}, C), 141.0\left(\mathrm{dm}, J_{C-\mathrm{F}}=253.1 \mathrm{~Hz}, C\right), 144.7\left(\mathrm{dm}, J_{C-\mathrm{F}}=247.3 \mathrm{~Hz}, C\right) .{ }^{19} \mathrm{~F}$ NMR $\left(369 \mathrm{MHz}, \mathrm{CDCl}_{3}\right.$, $\delta):-163.0(\mathrm{t}, J=25.6 \mathrm{~Hz}, 2 \mathrm{~F}),-155.8-155.7(\mathrm{~m}, 1 \mathrm{~F}),-140.7(\mathrm{~d}, J=22.9 \mathrm{~Hz}, 2 \mathrm{~F})$. HRMS-EI $(\mathrm{m} / \mathrm{z})$ : $[\mathrm{M}]^{+}$calcd for $\mathrm{C}_{22} \mathrm{H}_{9} \mathrm{~F}_{5}, 368.0624$; found, 368.0617 . 


\section{2,3,4,5,6-Pentafluoro-1,1':4',1":4",1"''-quaterphenyl (3p).}

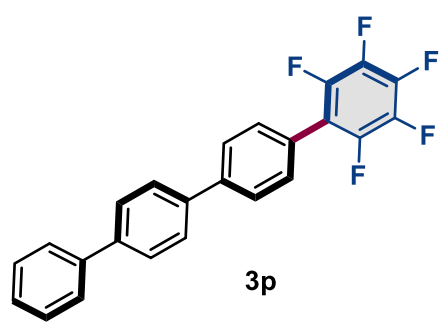

The reaction was carried out with $62.2 \mathrm{mg}(0.20 \mathrm{mmol})$ of $\mathbf{1 p}$ and $64.7 \mathrm{mg}(0.22 \mathrm{mmol})$ of $\mathbf{2 b}$ according to Procedure A. The product 3p was obtained as a white powder (73.2 $\mathrm{mg}, 0.18 \mathrm{mmol}, 92 \%$ yield) after purification by reprecipitation $\left(\mathrm{CH}_{2} \mathrm{Cl}_{2} / \mathrm{MeOH}\right) .{ }^{1} \mathrm{H}$ was in agreement with the literature. ${ }^{10}$ ${ }^{1} \mathrm{H}$ NMR (396 MHz, $\mathrm{CDCl}_{3}, \delta$ ): 7.33-7.39 (m, 1H), 7.46 (t, $\left.J=7.3 \mathrm{~Hz}, 2 \mathrm{H}\right), 7.51$ (d, $\left.J=7.9 \mathrm{~Hz}, 2 \mathrm{H}\right)$, 7.60-7.66 (m, 2H), 7.68-7.74 (m, 4H), 7.76 (d, $J=7.9 \mathrm{~Hz}, 2 \mathrm{H}) .{ }^{19} \mathrm{~F}$ NMR $\left(373 \mathrm{MHz}, \mathrm{CDCl}_{3}, \delta\right)$ :$163.4-163.3(\mathrm{~m}, 2 \mathrm{~F}),-156.7(\mathrm{t}, J=20.0 \mathrm{~Hz}, 1 \mathrm{~F}),-144.5(\mathrm{~d}, J=16.7 \mathrm{~Hz}, 2 \mathrm{~F}) .{ }^{13} \mathrm{C}$ NMR peaks were barely detected because of the low solubility of 3p. HRMS-EI $(\mathrm{m} / \mathrm{z})$ : $[\mathrm{M}]^{+}$calcd for $\mathrm{C}_{24} \mathrm{H}_{13} \mathrm{~F}_{5}$, 396.0937; found, 396.0925.

\section{2,3,4,5,6-Pentafluoro-4'-(phenylethynyl)-1,1'-biphenyl (3q).}

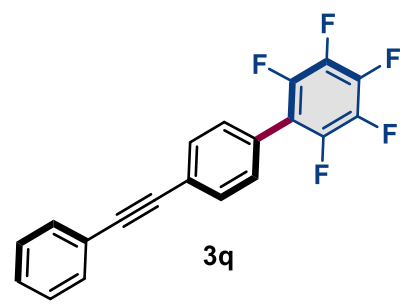

The reaction was carried out with $51.0 \mathrm{mg}(0.20 \mathrm{mmol})$ of $\mathbf{1 q}$ and $65.6 \mathrm{mg}(0.22 \mathrm{mmol})$ of $\mathbf{2} \mathbf{b}$ according to Procedure A. The product 3q was obtained as a white powder (43.6 mg, $0.13 \mathrm{mmol}, 64 \%$ yield) after purification by silica-gel column chromatography $\left(\mathrm{SiO}_{2}\right.$, hexane only).

${ }^{1} \mathrm{H}$ NMR $\left(396 \mathrm{MHz}, \mathrm{CDCl}_{3}, \delta\right): 7.35-7.40(\mathrm{~m}, 3 \mathrm{H}), 7.43(\mathrm{~d}, J=7.9 \mathrm{~Hz}, 2 \mathrm{H}), 7.55(\mathrm{~d}, J=2.4 \mathrm{~Hz}, 1 \mathrm{H})$, 7.56-7.59 (m, 1H), 7.65 (d, $J=8.3 \mathrm{~Hz}, 2 \mathrm{H}) .{ }^{13} \mathrm{C} \mathrm{NMR}\left(100 \mathrm{MHz}, \mathrm{CDCl}_{3}, \delta\right): 88.5(C), 90.9(C), 115.3$ $\left(\mathrm{td}, J_{C-\mathrm{F}}=1.2,16.8 \mathrm{~Hz}, C\right), 122.8(C), 124.4(C), 126.0(C), 128.4(\mathrm{CH}), 128.6(\mathrm{CH}), 130.1(\mathrm{CH})$, $131.7(C \mathrm{H}), 131.8(C \mathrm{H}), 137.8\left(\mathrm{dm}, J_{C-\mathrm{F}}=253.9 \mathrm{~Hz}, C\right), 140.4\left(\mathrm{dm}, J_{C-\mathrm{F}}=263.4 \mathrm{~Hz}, C\right), 144.0(\mathrm{dm}$, $\left.J_{C-\mathrm{F}}=245.3 \mathrm{~Hz}, C\right) .{ }^{19} \mathrm{~F}$ NMR $\left(373 \mathrm{MHz}, \mathrm{CDCl}_{3}, \delta\right):-163.2-163.1(\mathrm{~m}, 2 \mathrm{~F}),-156.2(\mathrm{t}, J=22.9 \mathrm{~Hz}$, $1 \mathrm{~F}),-144.3$ (dd, $J=11.6,22.8 \mathrm{~Hz}, 2 \mathrm{~F})$. HRMS-EI $(\mathrm{m} / \mathrm{z})$ : [M] ${ }^{+}$calcd for $\mathrm{C}_{20} \mathrm{H}_{9} \mathrm{~F}_{5}, 344.0624$; found, 344.0627. 
(E)-2,3,4,5,6-Pentafluoro-4'-styryl-1,1'-biphenyl (3r).

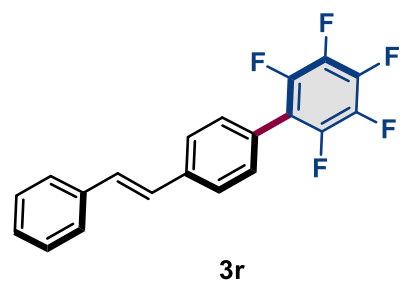

The reaction was carried out with $51.0 \mathrm{mg}(0.20 \mathrm{mmol})$ of $\mathbf{1 r}$ and $64.4 \mathrm{mg}(0.22 \mathrm{mmol})$ of $\mathbf{2 b}$ according to Procedure A. The product $3 \mathbf{r}$ was obtained as a white powder ( $42.0 \mathrm{mg}, 0.12 \mathrm{mmol}, 62 \%$ yield) after purification by silica-gel column chromatography $\left(\mathrm{SiO}_{2}\right.$, hexane only).

${ }^{1} \mathrm{H} \mathrm{NMR}\left(401 \mathrm{MHz}, \mathrm{CDCl}_{3}, \delta\right): 7.14(\mathrm{~d}, J=16.8 \mathrm{~Hz}, 1 \mathrm{H}), 7.21$ (d, $\left.J=16.0 \mathrm{~Hz}, 1 \mathrm{H}\right), 7.30$ (t, $J=7.4$ $\mathrm{Hz}, 1 \mathrm{H}), 7.39$ (t, $J=7.4 \mathrm{~Hz}, 2 \mathrm{H}), 7.43(\mathrm{~d}, J=8.4 \mathrm{~Hz}, 2 \mathrm{H}), 7.55$ (d, $J=7.2 \mathrm{~Hz}, 2 \mathrm{H}), 7.64$ (d, $J=8.4$ $\mathrm{Hz}, 2 \mathrm{H}) .{ }^{13} \mathrm{C} \mathrm{NMR}\left(100 \mathrm{MHz}, \mathrm{CDCl}_{3}, \delta\right): 115.8\left(\mathrm{td}, J_{C-\mathrm{F}}=3.8,17.2 \mathrm{~Hz}, C\right), 125.4(C), 126.7(\mathrm{CH})$, $127.7(\mathrm{CH}), 128.0(\mathrm{CH}), 128.8(\mathrm{CH}), 130.4(\mathrm{CH}), 130.5(\mathrm{CH}), 137.1(\mathrm{C}), 138.0\left(\mathrm{dm}, J_{C-\mathrm{F}}=253.8 \mathrm{~Hz}\right.$, C), $138.5(C), 140.5\left(\mathrm{dm}, J_{C-\mathrm{F}}=246.2 \mathrm{~Hz}, C\right), 144.3\left(\mathrm{dm}, J_{C-\mathrm{F}}=249.1 \mathrm{~Hz}, C\right) .{ }^{19} \mathrm{~F}$ NMR $(373 \mathrm{MHz}$, $\left.\mathrm{CDCl}_{3}, \delta\right):-163.4(\mathrm{t}, J=25.7 \mathrm{~Hz}, 2 \mathrm{~F}),-156.8(\mathrm{t}, J=20.0 \mathrm{~Hz}, 1 \mathrm{~F}),-144.5(\mathrm{~d}, J=17.2 \mathrm{~Hz}, 2 \mathrm{~F})$. HRMSEI $(m / z):[\mathrm{M}]^{+}$calcd for $\mathrm{C}_{20} \mathrm{H}_{11} \mathrm{~F}_{5}, 346.0781$; found, 346.0785 .

\section{2,3,4,5,6-Pentafluoro-4'-(1,2,2-triphenylvinyl)-1,1'-biphenyl (3s).}

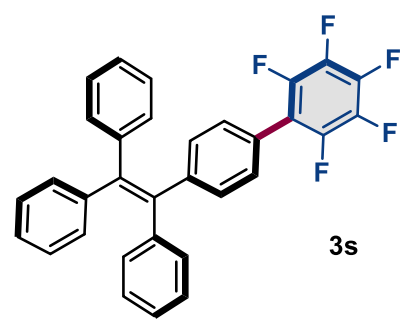

The reaction was carried out with $82.1 \mathrm{mg}(0.20 \mathrm{mmol})$ of $\mathbf{1 s}$ and $65.3 \mathrm{mg}(0.22 \mathrm{mmol})$ of $\mathbf{2 b}$ according to Procedure A. The product 3s was obtained as a white powder ( $40.5 \mathrm{mg}, 0.08 \mathrm{mmol}, 41 \%$ yield) after purification by silica-gel column chromatography $\left(\mathrm{SiO}_{2}\right.$, hexane only) and GPC.

${ }^{1} \mathrm{H}$ NMR (392 MHz, $\mathrm{CDCl}_{3}, \delta$ ): 7.02-7.09 (m, 6H), 7.10-7.19 (m, 13H). ${ }^{13} \mathrm{C}$ NMR (100 MHz, $\mathrm{CDCl}_{3}$,

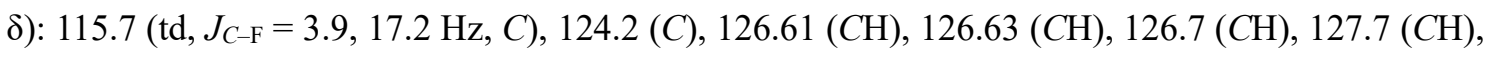
$127.8(\mathrm{CH}), 129.4(\mathrm{CH}), 131.26(\mathrm{CH}), 131.30(\mathrm{CH}), 131.35(\mathrm{CH}), 131.5(\mathrm{CH}), 137.8\left(\mathrm{dm}, J_{C-\mathrm{F}}=252.9\right.$ $\mathrm{Hz}, C), 140.0(C), 140.2\left(\mathrm{dm}, J_{C-\mathrm{F}}=231.9 \mathrm{~Hz}, C\right), 142.0$ (C), 143.2 (C), 143.3 (C), $143.4(C), 144.1$ $\left(\mathrm{dm}, J_{C-\mathrm{F}}=248.1 \mathrm{~Hz}, C\right), 144.8(C) .{ }^{19} \mathrm{~F} \mathrm{NMR}\left(369 \mathrm{MHz}, \mathrm{CDCl}_{3}, \delta\right):-163.7-163.6(\mathrm{~m}, 2 \mathrm{~F}),-157.2-$ $-157.1(\mathrm{~m}, 1 \mathrm{~F}),-144.4(\mathrm{dd}, J=9.2,22.5 \mathrm{~Hz}, 2 \mathrm{~F})$. HRMS-EI $(\mathrm{m} / \mathrm{z})$ : [M] ${ }^{+}$calcd for $\mathrm{C}_{32} \mathrm{H}_{1}{ }_{9} \mathrm{~F}_{5}, 498.1407$; found, 498.1390 . 


\section{3-(Perfluorophenyl)-9-phenyl-9H-carbazole (3t).}

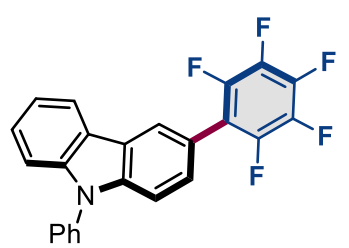

$3 t$

The reaction was carried out with $64.5 \mathrm{mg}(0.20 \mathrm{mmol})$ of $\mathbf{1 t}$ and $88.0 \mathrm{mg}(0.30 \mathrm{mmol})$ of $\mathbf{2 b}$ according to Procedure A. The product $3 \mathrm{t}$ was obtained as a orange powder ( $66.5 \mathrm{mg}, 0.16 \mathrm{mmol}, 81 \%$ yield) after purification by silica-gel column chromatography $\left(\mathrm{SiO}_{2}, \mathrm{CH}_{2} \mathrm{Cl}_{2} /\right.$ hexane, 0:100-25:75).

${ }^{1} \mathrm{H}$ NMR $\left(396 \mathrm{MHz}, \mathrm{CDCl}_{3}, \delta\right): 7.33$ (ddd, $\left.J=1.7,6.2,7.8 \mathrm{~Hz}, 1 \mathrm{H}\right), 7.41-7.47$ (m, 3H), 7.48-7.54 (m, 2H), 7.57-7.67 (m, 4H), $8.16(\mathrm{~d}, J=7.9 \mathrm{~Hz}, 1 \mathrm{H}), 8.21(\mathrm{~s}, 1 \mathrm{H}) .{ }^{13} \mathrm{C} \mathrm{NMR}\left(99 \mathrm{MHz}, \mathrm{CDCl}_{3}, \delta\right): 110.0$ $(C H), 116.7\left(\mathrm{td}, J_{C-\mathrm{F}}=4.1,17.2 \mathrm{~Hz}, C\right), 117.4(C), 120.37(\mathrm{CH}), 120.41(\mathrm{CH}), 122.3(\mathrm{CH}), 122.9(C)$, $123.6(C), 126.5(C H), 127.0(C H), 127.7(C H), 127.8(C H), 130.0(C H), 137.2(C), 137.8\left(\mathrm{dm}, J_{C-\mathrm{F}}\right.$ $=254.0 \mathrm{~Hz}, C), 138.7(\mathrm{~m}, \mathrm{C}), 140.9(C), 141.3(C), 144.3\left(\mathrm{dm}, J_{C-\mathrm{F}}=252.2 \mathrm{~Hz}, C\right) .{ }^{19} \mathrm{~F}$ NMR $(373$ $\left.\mathrm{MHz}, \mathrm{CDCl}_{3}, \delta\right):-163.8(\mathrm{t}, J=23.1 \mathrm{~Hz}, 2 \mathrm{~F}),-157.9(\mathrm{t}, J=20.0 \mathrm{~Hz}, 1 \mathrm{~F}),-144.7(\mathrm{dd}, J=11.6,22.8$ $\mathrm{Hz}, 2 \mathrm{~F})$. HRMS-EI $(\mathrm{m} / \mathrm{z})$ : [M] $]^{+}$calcd for $\mathrm{C}_{24} \mathrm{H}_{12} \mathrm{~F}_{5} \mathrm{~N}_{1}, 409.0890$; found, 409.0871 .

\section{5-(Perfluorophenyl)-2,2'-bithiophene (3u).}

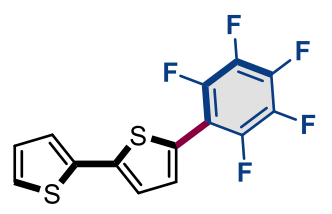

$3 \mathbf{u}$

The reaction was carried out with $49.3 \mathrm{mg}(0.20 \mathrm{mmol})$ of $\mathbf{1 u}$ and $65.6 \mathrm{mg}(0.22 \mathrm{mmol})$ of $\mathbf{2 b}$ according to Procedure A. The product $3 \mathbf{u}$ was obtained as a yellow-green powder $(48.7 \mathrm{mg}, 0.15 \mathrm{mmol}, 73 \%$ yield) after purification by silica-gel column chromatography $\left(\mathrm{SiO}_{2}\right.$, hexane only).

${ }^{1} \mathrm{H}$ NMR (396 MHz, $\left.\mathrm{CDCl}_{3}, \delta\right): 7.06(\mathrm{dd}, J=3.8,5.0 \mathrm{~Hz}, 1 \mathrm{H}), 7.23$ (d, $\left.J=4.0 \mathrm{~Hz}, 4 \mathrm{H}\right), 7.25-7.27$ (m, $2 \mathrm{H}), 7.29(\mathrm{dd}, J=1.0,5.3 \mathrm{~Hz} 1 \mathrm{H}), 7.46(\mathrm{~d}, J=3.6 \mathrm{~Hz}, 1 \mathrm{H}) .{ }^{13} \mathrm{C} \mathrm{NMR}\left(100 \mathrm{MHz}, \mathrm{CDCl}_{3}, \delta\right): 109.8$ $\left(\operatorname{td}, J_{C-\mathrm{F}}=3.8,15.3 \mathrm{~Hz}, C\right), 123.7(\mathrm{CH}), 124.5(\mathrm{CH}), 124.9(\mathrm{C}), 125.4(\mathrm{CH}), 128.0(\mathrm{CH}), 130.9\left(\mathrm{t}, J_{C-}\right.$ $\mathrm{F}=5.8 \mathrm{~Hz}, C \mathrm{H}), 136.2(C), 137.7\left(\mathrm{dm}, J_{C-\mathrm{F}}=155.2 \mathrm{~Hz}, C\right), 140.18\left(\mathrm{t}, J_{C-\mathrm{F}}=3.8 \mathrm{~Hz}, C\right), 140.21(\mathrm{dm}$, $\left.J_{C-\mathrm{F}}=158.1 \mathrm{~Hz}, C\right), 143.9\left(\mathrm{dm}, J_{C-\mathrm{F}}=252.0 \mathrm{~Hz}, C\right) .{ }^{19} \mathrm{~F} \mathrm{NMR}\left(373 \mathrm{MHz}, \mathrm{CDCl}_{3}, \delta\right):-163.2(\mathrm{t}, J=$ $22.9 \mathrm{~Hz}, 2 \mathrm{~F}),-157.2-157.1(\mathrm{~m}, 1 \mathrm{~F}),-141.0(\mathrm{~d}, J=17.2 \mathrm{~Hz}, 2 \mathrm{~F})$. HRMS-EI $(\mathrm{m} / \mathrm{z})$ : $[\mathrm{M}]^{+}$calcd for $\mathrm{C}_{14} \mathrm{H}_{5} \mathrm{~F}_{5} \mathrm{~S}_{2}, 331.9753$; found, 331.9753 . 
9,10-Bis(perfluorophenyl)anthracene (3v).<smiles>Fc1c(F)c(F)c(-c2c3ccccc3c(-c3c(F)c(F)c(F)c(F)c3F)c3ccccc23)c(F)c1F</smiles>

3v

The reaction was carried out with $50.5 \mathrm{mg}(0.15 \mathrm{mmol})$ of $\mathbf{1 v}$ and $110.3 \mathrm{mg}(0.375 \mathrm{mmol})$ of $\mathbf{2 b}$ according to Procedure B. The product $3 \mathbf{v}$ was obtained as a yellow-green powder $(46.7 \mathrm{mg}, 0.09$ mmol, $61 \%$ yield) after purification by silica-gel column chromatography $\left(\mathrm{SiO}_{2}, \mathrm{CH}_{2} \mathrm{Cl}_{2} /\right.$ hexane, 0:100-10:90).

${ }^{1} \mathrm{H}$ NMR (396 MHz, $\left.\mathrm{CDCl}_{3}, \delta\right): 7.51-7.56$ (m, 4H), 7.60-7.65 (m, 4H). ${ }^{13} \mathrm{C}$ NMR (99 MHz, $\mathrm{CDCl}_{3}$,

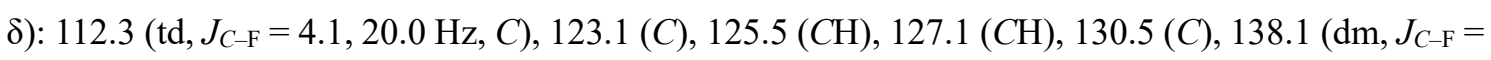
$255.8 \mathrm{~Hz}, C), 141.8\left(\mathrm{dm}, J_{C-\mathrm{F}}=252.1 \mathrm{~Hz}, C\right), 145.1\left(\mathrm{dm}, J_{C-\mathrm{F}}=250.2 \mathrm{~Hz}, C\right) .{ }^{19} \mathrm{~F}$ NMR $(369 \mathrm{MHz}$, $\left.\mathrm{CDCl}_{3}, \delta\right):-162.2-162.1(\mathrm{~m}, 4 \mathrm{~F}),-154.0(\mathrm{t}, J=19.9 \mathrm{~Hz}, 2 \mathrm{~F}),-138.9(\mathrm{dd}, J=9.6,21.0 \mathrm{~Hz}, 4 \mathrm{~F})$. HRMS-EI $(\mathrm{m} / \mathrm{z})$ : [M] $]^{+}$calcd for $\mathrm{C}_{26} \mathrm{H}_{8} \mathrm{~F}_{10}, 510.0466$; found, 510.0470 .

\section{2,7-bis(perfluorophenyl)-9,9'-spirobi[fluorene] (3w).}

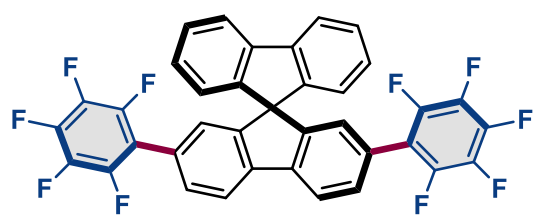

$3 w$

The reaction was carried out with $71.4 \mathrm{mg}(0.15 \mathrm{mmol})$ of $\mathbf{1 w}$ and $111.2 \mathrm{mg}(0.375 \mathrm{mmol})$ of $\mathbf{2 b}$ according to Procedure B. The product $\mathbf{3 w}$ was obtained as a white powder $(51.0 \mathrm{mg}, 0.08 \mathrm{mmol}$, $52 \%$ yield) after purification by silica-gel column chromatography $\left(\mathrm{SiO}_{2}, \mathrm{CH}_{2} \mathrm{Cl}_{2} /\right.$ hexane, 0:100-10:90) and GPC.

${ }^{1} \mathrm{H}$ NMR $\left(392 \mathrm{MHz}, \mathrm{CDCl}_{3}, \delta\right): 6.79-6.83(\mathrm{~m}, 4 \mathrm{H}), 7.15(\mathrm{td}, J=1.0,7.4 \mathrm{~Hz}, 2 \mathrm{H}), 7.39$ (td, $J=0.9$, $7.6 \mathrm{~Hz}, 2 \mathrm{H}), 7.47(\mathrm{dd}, J=1.4,7.6 \mathrm{~Hz}, 2 \mathrm{H}), 7.84(\mathrm{~d}, J=7.8 \mathrm{~Hz}, 2 \mathrm{H}), 8.00(\mathrm{~d}, J=8.2 \mathrm{~Hz}, 2 \mathrm{H}) .{ }^{13} \mathrm{C}$ NMR (99 MHz, $\left.\mathrm{CDCl}_{3}, \delta\right): 65.9(C), 115.7\left(\mathrm{td}, J_{C-\mathrm{F}}=3.8,17.0 \mathrm{~Hz}, C\right), 120.2(\mathrm{CH}), 120.6(C \mathrm{H}), 124.0$ $(C \mathrm{H}), 126.0(\mathrm{CH}), 126.3(\mathrm{C}), 128.0(\mathrm{CH}), 128.1(\mathrm{CH}), 130.0(\mathrm{CH}), 137.7\left(\mathrm{dm}, J_{C-\mathrm{F}}=253.0 \mathrm{~Hz}, C\right)$, $140.2\left(\mathrm{dm}, J_{C-\mathrm{F}}=249.3 \mathrm{~Hz}, C\right), 141.7(C), 141.8(C), 143.4(C), 144.0\left(\mathrm{dm}, J_{C-\mathrm{F}}=249.2 \mathrm{~Hz}, C\right), 147.4$ (C), $149.8(C) .{ }^{19} \mathrm{~F}$ NMR (369 MHz, $\left.\mathrm{CDCl}_{3}, \delta\right):-163.6-163.5(\mathrm{~m}, 4 \mathrm{~F}),-156.8(\mathrm{t}, J=19.9 \mathrm{~Hz}, 2 \mathrm{~F}),-$ 144.1 (d, $J=22.5 \mathrm{~Hz}, 4 \mathrm{~F})$. HRMS-EI $(\mathrm{m} / \mathrm{z})$ : [M] $]^{+}$calcd for $\mathrm{C}_{37} \mathrm{H}_{14} \mathrm{~F}_{10}, 648.0936$; found, 648.0912. 
2,2"'",3,3"'",4,4"'",5,5"',,6,6"'"-Decafluoro-2",3",5",6"-tetraphenyl-1,1':4',1":4",1"':4"',1"'"quinquephenyl (3x).

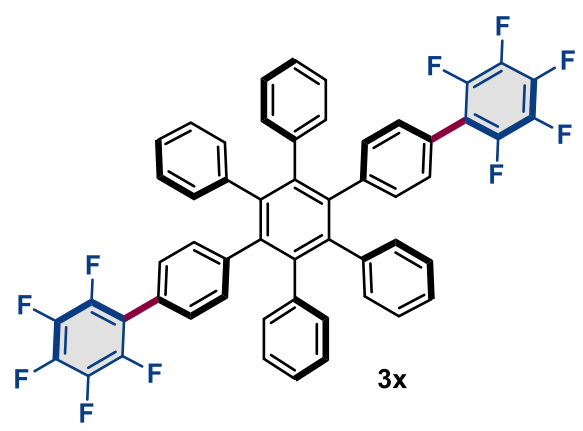

The reaction was carried out with $69.2 \mathrm{mg}(0.10 \mathrm{mmol})$ of $\mathbf{1 x}$ and $88.5 \mathrm{mg}(0.30 \mathrm{mmol})$ of $\mathbf{2} \mathbf{b}$ according to Procedure C. The product 3x was obtained as a yellow powder (55.6 mg, $0.06 \mathrm{mmol}, 64 \%$ yield) after purification by silica-gel column chromatography $\left(\mathrm{SiO}_{2}, \mathrm{CH}_{2} \mathrm{Cl}_{2} /\right.$ hexane, 0:100-30:70) and reprecipitation $\left(\mathrm{CH}_{2} \mathrm{Cl}_{2} / \mathrm{MeOH}\right)$.

${ }^{1} \mathrm{H}$ NMR (401 MHz, $\left.\mathrm{CDCl}_{3}, \delta\right): 6.80-6.96(\mathrm{~m}, 28 \mathrm{H}) .{ }^{13} \mathrm{C} \mathrm{NMR}\left(99 \mathrm{MHz}, \mathrm{CDCl}_{3}, \delta\right): 115.9\left(\mathrm{td}, J_{C-\mathrm{F}}=\right.$ 3.5, $17.3 \mathrm{~Hz}, C), 123.1(C), 125.5(C \mathrm{H}), 126.8(C \mathrm{H}), 128.4(C \mathrm{H}), 131.4(\mathrm{CH}), 131.6(\mathrm{CH}), 137.6(\mathrm{dm}$, $\left.J_{C-\mathrm{F}}=252.1 \mathrm{~Hz}, C\right), 139.9(C), 140.08(C), 140.09\left(\mathrm{dm}, J_{C-\mathrm{F}}=235.1 \mathrm{~Hz}, C\right), 140.4(C), 141.9(C)$, $144.0\left(\mathrm{dm}, J_{C-\mathrm{F}}=248.3 \mathrm{~Hz}, C\right) .{ }^{19} \mathrm{~F}$ NMR $\left(373 \mathrm{MHz}, \mathrm{CDCl}_{3}, \delta\right):-164.0(\mathrm{td}, J=7.3,22.8 \mathrm{~Hz}, 4 \mathrm{~F}),-$ $157.6(\mathrm{t}, J=20.3 \mathrm{~Hz}, 2 \mathrm{~F}),-144.5(\mathrm{dd}, J=7.8,23.5 \mathrm{~Hz}, 4 \mathrm{~F})$. HRMS-ESI $(m / z):[\mathrm{M}+\mathrm{Na}]^{+}$calcd for $\mathrm{C}_{54} \mathrm{H}_{28} \mathrm{~F}_{10} \mathrm{Na}, 889.1924$; found, 889.1931.

\section{5,5"-Bis(perfluorophenyl)-2,2':5',2"'-terthiophene (3y).}

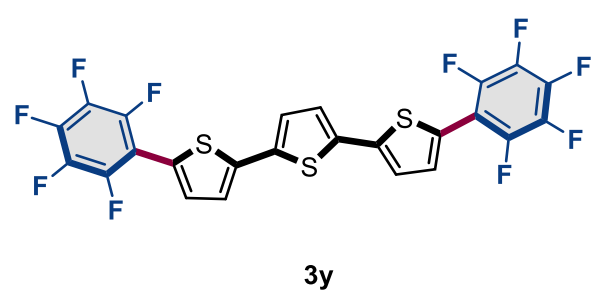

The reaction was carried out with $60.7 \mathrm{mg}(0.15 \mathrm{mmol})$ of $\mathbf{1 y}$ and $110.2 \mathrm{mg}(0.375 \mathrm{mmol})$ of $\mathbf{2 b}$ according to Procedure B. The product 3y was obtained as a yellow powder $(69.2 \mathrm{mg}, 0.12 \mathrm{mmol}$, $80 \%$ yield) after purification by silica-gel column chromatography $\left(\mathrm{SiO}_{2}, \mathrm{CH}_{2} \mathrm{Cl}_{2} /\right.$ hexane, 0:100-100:0) and reprecipitation $\left(\mathrm{CH}_{2} \mathrm{Cl}_{2} / \mathrm{MeOH}\right) .{ }^{1} \mathrm{H}$ and ${ }^{13} \mathrm{C}$ NMR were in agreement with the literature. ${ }^{11}$

${ }^{1} \mathrm{H}$ NMR (396 MHz, $\mathrm{CDCl}_{3}, \delta$ ): 7.19 (s, 2H), 7.24 (s, 2H), 7.47 (d, $\left.J=3.6 \mathrm{~Hz}, 2 \mathrm{H}\right) .{ }^{19} \mathrm{~F}$ NMR (369 $\left.\mathrm{MHz}, \mathrm{CDCl}_{3}, \delta\right):-163.1(\mathrm{td}, J=6.3,21.8 \mathrm{~Hz}, 4 \mathrm{~F}),-156.8(\mathrm{t}, J=21.8 \mathrm{~Hz}, 2 \mathrm{~F}),-140.8(\mathrm{dd}, J=5.9$, $21.8 \mathrm{~Hz}, 4 \mathrm{~F}) .{ }^{13} \mathrm{C}$ NMR peaks were barely detected because of the low solubility of $\mathbf{3 y}$. HRMS-EI $(\mathrm{m} / \mathrm{z}):[\mathrm{M}]^{+}$calcd for $\mathrm{C}_{24} \mathrm{H}_{6} \mathrm{~F}_{10} \mathrm{~S}_{3}, 579.9472$; found, 579.9470 . 


\section{1,3,6,8-Tetrakis(perfluorophenyl)pyrene (3z).}

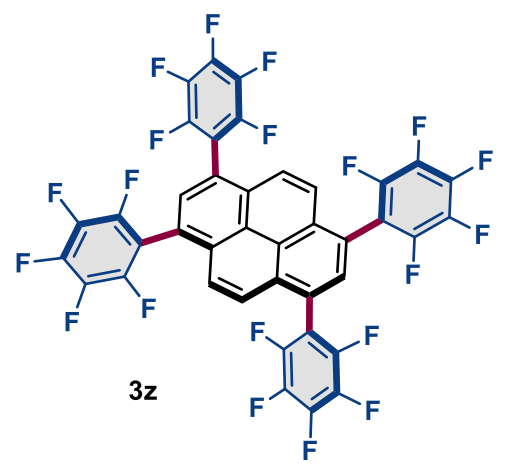

The reaction was carried out with $26.3 \mathrm{mg}(0.05 \mathrm{mmol})$ of $\mathbf{1 z}$ and $89.0 \mathrm{mg}(0.30 \mathrm{mmol})$ of $\mathbf{2} \mathbf{b}$ according to Procedure D. The product $\mathbf{3 z}$ was obtained as a white powder (11.2 $\mathrm{mg}, 0.01 \mathrm{mmol}, 25 \%$ yield) after purification by silica-gel column chromatography $\left(\mathrm{SiO}_{2}, \mathrm{CH}_{2} \mathrm{Cl}_{2} /\right.$ hexane, 0:100-100:0) and GPC. ${ }^{1} \mathrm{H} \mathrm{NMR}\left(392 \mathrm{MHz}, \mathrm{CDCl}_{3}, \delta\right.$ ): 7.96 (s, 4H), 8.01 (s, 2H). ${ }^{19} \mathrm{~F} \mathrm{NMR} \mathrm{(369} \mathrm{MHz,} \mathrm{CDCl}_{3}, \delta$ ): - 162.0 (td, $J=9.2,21.7 \mathrm{~Hz}, 8 \mathrm{~F}),-153.8(\mathrm{t}, J=20.2 \mathrm{~Hz}, 4 \mathrm{~F}),-140.1(\mathrm{dd}, J=7.9,23.3 \mathrm{~Hz}, 8 \mathrm{~F}) .{ }^{13} \mathrm{C}$ NMR peaks were barely detected because of the low solubility of $\mathbf{3 z}$. HRMS-EI $(\mathrm{m} / \mathrm{z})$ : $[\mathrm{M}]^{+}$calcd for $\mathrm{C}_{40} \mathrm{H}_{6} \mathrm{~F}_{20}$, 866.0150; found, 866.0125. 3z was also characterized by single X-ray structure analysis.

\section{2-(Perfluorophenyl)naphthalene (3aa).}

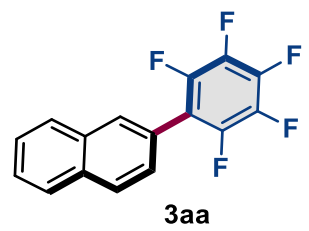

The reaction was carried out with $41.2 \mathrm{mg}(0.20 \mathrm{mmol})$ of $\mathbf{1}$ aa and $47.1 \mathrm{mg}(0.22 \mathrm{mmol})$ of $\mathbf{2 a}$ according to Procedure E. The product 3aa was obtained as a white powder (44.9 mg, $0.15 \mathrm{mmol}$, $77 \%$ yield) after purification by silica-gel column chromatography $\left(\mathrm{SiO}_{2}\right.$, hexane only). ${ }^{1} \mathrm{H}$ and ${ }^{13} \mathrm{C}$ NMR were in agreement with the literature. ${ }^{12}$

${ }^{1} \mathrm{H}$ NMR (392 MHz, $\mathrm{CDCl}_{3}, \delta$ ): 7.50 (dq, $\left.J=1.7,7.8 \mathrm{~Hz}, 1 \mathrm{H}\right), 7.53-7.60$ (m, 2H), 7.87-7.99 (m, 4H). ${ }^{13} \mathrm{C}$ NMR (99 MHz, $\left.\mathrm{CDCl}_{3}, \delta\right): 116.1\left(\mathrm{td}, J_{C-\mathrm{F}}=3.5,17.7 \mathrm{~Hz}, C\right), 123.8(\mathrm{C}), 126.7(\mathrm{CH}), 127.0(\mathrm{CH})$, 127.2 (CH), $127.8(\mathrm{CH}), 128.3(\mathrm{CH}), 128.4(\mathrm{CH}), 130.1(\mathrm{CH}), 133.1(\mathrm{C}), 133.3(C), 138.0\left(\mathrm{dm}, J_{C-\mathrm{F}}\right.$ $=251.2 \mathrm{~Hz}, C), 140.5\left(\mathrm{dm}, J_{C-\mathrm{F}}=254.9 \mathrm{~Hz}, C\right), 144.4\left(\mathrm{dm}, J_{C-\mathrm{F}}=249.3 \mathrm{~Hz}, C\right) .{ }^{19} \mathrm{~F}$ NMR $(369 \mathrm{MHz}$, $\left.\mathrm{CDCl}_{3}, \delta\right):-163.4(\mathrm{td}, J=6.3,21.8 \mathrm{~Hz}, 2 \mathrm{~F}),-156.7(\mathrm{t}, J=21.8 \mathrm{~Hz}, 1 \mathrm{~F}),-144.3(\mathrm{dd}, J=7.7,23.2 \mathrm{~Hz}$, 2F). HRMS-EI $(m / z):[M]^{+}$calcd for $\mathrm{C}_{16} \mathrm{H}_{7} \mathrm{~F}_{5}, 294.0468$; found, 294.0459 . 


\section{2-(2,3,5,6-Tetrafluoro-4-isopropoxyphenyl)naphthalene (3ab).}

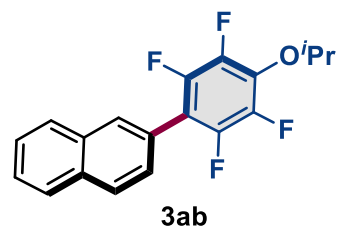

The reaction was carried out with $42.3 \mathrm{mg}(0.20 \mathrm{mmol})$ of $1 \mathbf{a a}$ and $75.8 \mathrm{mg}(0.30 \mathrm{mmol})$ of $\mathbf{2 c}$ according to Procedure E. The product 3ab was obtained as a yellow powder $(65.2 \mathrm{mg}, 0.20 \mathrm{mmol}$, $95 \%$ yield) after purification by silica-gel column chromatography $\left(\mathrm{SiO}_{2}\right.$, EtOAc/hexane, 0:100-4:96). ${ }^{1} \mathrm{H}$ NMR (400 MHz, $\mathrm{CDCl}_{3}, \delta$ ): 1.43 (d, $\left.J=6.0 \mathrm{~Hz}, 6 \mathrm{H}\right), 4.62$ (s, $\left.J=6.3 \mathrm{~Hz}, 1 \mathrm{H}\right), 7.51-7.58$ (m, 3H), 7.88-7.99 (m, 4H). $\left.{ }^{13} \mathrm{C} \mathrm{NMR} \mathrm{(101} \mathrm{MHz,} \mathrm{CDCl}_{3}, \delta\right): 22.4\left(\mathrm{CH}_{3}\right), 78.3(\mathrm{CH}), 114.6\left(\mathrm{t}, J_{C-\mathrm{F}}=17.0 \mathrm{~Hz}\right.$, C), $124.7(C), 126.5(\mathrm{CH}), 126.9(\mathrm{CH}), 127.3(\mathrm{CH}), 127.7(\mathrm{CH}), 128.15(\mathrm{CH}), 128.25(\mathrm{CH}), 130.0$ $(C \mathrm{H}), 135.7\left(\mathrm{t}, J_{C-\mathrm{F}}=13.1 \mathrm{~Hz}, C\right), 142.1\left(\mathrm{dm}, J_{C-\mathrm{F}}=242.9 \mathrm{~Hz}, C\right), 144.4\left(\mathrm{dm}, J_{C-\mathrm{F}}=247.9 \mathrm{~Hz}, C\right)$. ${ }^{19} \mathrm{~F}$ NMR (376 MHz, $\left.\mathrm{CDCl}_{3}, \delta\right)$ : -157.7-157.6 (m, 2F), -146.53-146.48 (m, 2F). HRMS-EI $(\mathrm{m} / \mathrm{z})$ : $[\mathrm{M}]^{+}$calcd for $\mathrm{C}_{19} \mathrm{H}_{14} \mathrm{~F}_{4} \mathrm{O}_{1}, 334.0981$; found, 334.0980.

\section{2-(2,4,6-Trifluorophenyl)naphthalene (3ac).}

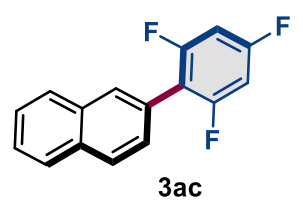

The reaction was carried out with $41.5 \mathrm{mg}(0.20 \mathrm{mmol})$ of $\mathbf{1}$ aa and $53.7 \mathrm{mg}(0.30 \mathrm{mmol})$ of $\mathbf{2 d}$ according to Procedure F. The product 3ac was obtained as a white powder $(38.1 \mathrm{mg}, 0.15 \mathrm{mmol}$, $74 \%$ yield) after purification by silica-gel column chromatography $\left(\mathrm{SiO}_{2}\right.$, hexane only). ${ }^{1} \mathrm{H}$ and ${ }^{13} \mathrm{C}$ NMR were in agreement with the literature. ${ }^{13}$

${ }^{1} \mathrm{H}$ NMR (400 MHz, $\left.\mathrm{CDCl}_{3}, \delta\right): 6.76-6.83(\mathrm{~m}, 2 \mathrm{H}), 7.49-7.56$ (m, 3H), $7.88(\mathrm{dt}, J=2.3,6.9 \mathrm{~Hz}, 2 \mathrm{H})$, 7.90-7.93 (m, 2H). ${ }^{13} \mathrm{C}$ NMR $\left(101 \mathrm{MHz}, \mathrm{CDCl}_{3}, \delta\right): 100.2-100.8(\mathrm{~m}, C \mathrm{H}), 115.0\left(\mathrm{td}, J_{C-\mathrm{F}}=4.8,18.9\right.$ Hz, C), $125.7(C), 126.3(C H), 126.6(C H), 127.6(C H), 127.7(C H), 127.9(C H), 128.2(C H), 129.8$ $(C \mathrm{H}), 132.9(C), 133.1(C), 160.4\left(\mathrm{ddd}, J_{C-\mathrm{F}}=9.7,14.5,250.7 \mathrm{~Hz}, C\right), 161.8\left(\mathrm{dt}, J_{C-\mathrm{F}}=15.5,250.7\right.$ $\mathrm{Hz}, C) .{ }^{19} \mathrm{~F}$ NMR $\left(376 \mathrm{MHz}, \mathrm{CDCl}_{3}, \delta\right):-112.4$ (t, $\left.J=6.8 \mathrm{~Hz}, 2 \mathrm{~F}\right),-110.2-110.1$ (m, 1F). HRMS-EI $(\mathrm{m} / \mathrm{z})$ : $[\mathrm{M}]^{+}$calcd for $\mathrm{C}_{16} \mathrm{H}_{9} \mathrm{~F}_{3}, 258.0656$; found, 258.0650 . 


\section{2-(2,3,5-Trifluorophenyl)naphthalene (3ad).}

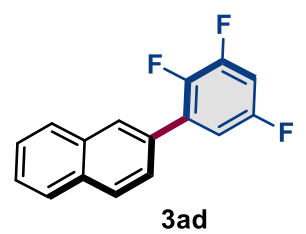

The reaction was carried out with $41.3 \mathrm{mg}(0.20 \mathrm{mmol})$ of $1 \mathbf{a a}$ and $53.0 \mathrm{mg}(0.50 \mathrm{mmol})$ of $\mathbf{2 e}$ according to Procedure E. The product 3ad was obtained as a white powder $(42.3 \mathrm{mg}, 0.16 \mathrm{mmol}$, $82 \%$ yield) after purification by silica-gel column chromatography $\left(\mathrm{SiO}_{2}\right.$, hexane only).

${ }^{1} \mathrm{H}$ NMR (400 MHz, $\mathrm{CDCl}_{3}, \delta$ ): 6.91-7.00 (m, 1H), 7.04-7.09 (m, 1H), 7.52-7.57 (m, 2H), 7.63 (dt, $J=1.9,8.7 \mathrm{~Hz}, 1 \mathrm{H}), 7.86-7.96(\mathrm{~m}, 3 \mathrm{H}), 8.01(\mathrm{~s}, 1 \mathrm{H}) .{ }^{13} \mathrm{C} \mathrm{NMR}\left(101 \mathrm{MHz}, \mathrm{CDCl}_{3}, \delta\right): 104.3\left(\mathrm{dd}, J_{\mathrm{C}-}\right.$ $\mathrm{F}=21.8,27.5 \mathrm{~Hz}, C \mathrm{H}), 111.8\left(\mathrm{~d}, J_{C-\mathrm{F}}=24.1 \mathrm{~Hz}, C \mathrm{H}\right), 126.19(\mathrm{CH}), 126.22(C \mathrm{H}), 126.6(C \mathrm{H}), 126.8$ $(\mathrm{CH}), 127.7(\mathrm{CH}), 128.3(\mathrm{CH}), 128.4(\mathrm{CH}), 131.2(\mathrm{C}), 131.7$ (t, $\left.J_{C-\mathrm{F}}=10.7 \mathrm{~Hz}, C\right), 133.0(C), 133.2$ (C), $144.8\left(\mathrm{ddd}, J_{C-\mathrm{F}}=3.9,13.6,246.8 \mathrm{~Hz}, C\right), 150.9\left(\mathrm{ddd}, J_{C-\mathrm{F}}=13.5,15.5,251.6 \mathrm{~Hz}, C\right), 157.6$ (ddd, $\left.J_{C-\mathrm{F}}=2.9,11.1,246.3 \mathrm{~Hz}, C\right) .{ }^{19} \mathrm{~F} \mathrm{NMR}\left(376 \mathrm{MHz}, \mathrm{CDCl}_{3}, \delta\right):-149.6-149.5(\mathrm{~m}, 1 \mathrm{~F}),-134.3$ (dd, $J$ $=9.8,20.7 \mathrm{~Hz}, 1 \mathrm{~F}),-116.8-116.7(\mathrm{~m}, 1 \mathrm{~F})$. HRMS-EI $(\mathrm{m} / \mathrm{z})$ : $[\mathrm{M}]^{+}$calcd for $\mathrm{C}_{16} \mathrm{H}_{9} \mathrm{~F}_{3}, 258.0656$; found, 258.0646 .

\section{2-(2,6-Difluorophenyl)naphthalene (3ae).}

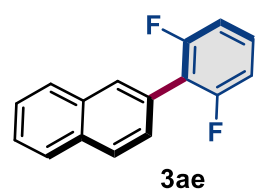

The reaction was carried out with $41.6 \mathrm{mg}(0.20 \mathrm{mmol})$ of 1 aa and $47.5 \mathrm{mg}(0.30 \mathrm{mmol})$ of $\mathbf{2 f}$ according to Procedure E. The product 3ae was obtained as a white powder (46.3 mg, $0.19 \mathrm{mmol}$, $96 \%$ yield) after purification by silica-gel column chromatography $\left(\mathrm{SiO}_{2}\right.$, hexane only). ${ }^{1} \mathrm{H}$ and ${ }^{13} \mathrm{C}$ NMR were in agreement with the literature. ${ }^{12}$

${ }^{1} \mathrm{H}$ NMR (400 MHz, $\left.\mathrm{CDCl}_{3}, \delta\right): 7.03$ (t, $\left.J=8.0 \mathrm{~Hz}, 2 \mathrm{H}\right), 7.28-7.37$ (m, 1H), 7.49-7.55 (m, 2H), 7.57 (dq, $J=1.6,8.8 \mathrm{~Hz}, 1 \mathrm{H}), 7.86-7.91(\mathrm{~m}, 2 \mathrm{H}), 7.93(\mathrm{~d}, J=8.4 \mathrm{~Hz}, 1 \mathrm{H}), 7.97(\mathrm{~s}, 1 \mathrm{H}) .{ }^{13} \mathrm{C}$ NMR $(101$ $\left.\mathrm{MHz}, \mathrm{CDCl}_{3}, \delta\right): 111.5-111.8(\mathrm{~m}, \mathrm{CH}), 118.5(C), 126.2(\mathrm{CH}), 126.5(\mathrm{CH}), 126.6(C), 127.66(\mathrm{CH})$, $127.75(\mathrm{CH}), 127.79(\mathrm{CH}), 128.2(\mathrm{CH}), 128.9\left(\mathrm{t}, J_{C-\mathrm{F}}=10.2 \mathrm{~Hz}, \mathrm{C}\right), 129.8(\mathrm{CH}), 132.9(\mathrm{C}), 133.1(\mathrm{C})$, $160.2\left(\mathrm{dd}, J_{C-\mathrm{F}}=6.8,249.7 \mathrm{~Hz}, C\right) .{ }^{19} \mathrm{~F} \mathrm{NMR}\left(376 \mathrm{MHz}, \mathrm{CDCl}_{3}, \delta\right):-115.7(\mathrm{t}, J=1.7 \mathrm{~Hz}, 2 \mathrm{~F})$. HRMSEI $(m / z)$ : [M] $]^{+}$calcd for $\mathrm{C}_{16} \mathrm{H}_{10} \mathrm{~F}_{2}, 240.0751$; found, 240.0743 . 


\section{2-(2,6-Difluoro-4-methoxyphenyl)naphthalene (3af).}

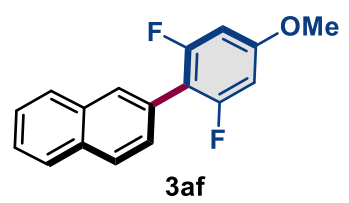

The reaction was carried out with $41.1 \mathrm{mg}(0.20 \mathrm{mmol})$ of $\mathbf{1}$ aa and $56.9 \mathrm{mg}(0.30 \mathrm{mmol})$ of $\mathbf{2 g}$ according to Procedure E. The product 3af was obtained as a yellow powder $(52.1 \mathrm{mg}, 0.19 \mathrm{mmol}$, 97\% yield) after purification by silica-gel column chromatography $\left(\mathrm{SiO}_{2}\right.$, EtOAc/hexane, 0:100-6:94). ${ }^{1} \mathrm{H}$ NMR (392 MHz, $\left.\mathrm{CDCl}_{3}, \delta\right): 3.84$ (s, 3H), 6.54-6.63 (m, 2H), 7.47-7.52 (m, 2H), 7.54 (dd, $J=1.2$, $8.6 \mathrm{~Hz}, 1 \mathrm{H}), 7.84-7.94(\mathrm{~m}, 4 \mathrm{H}) .{ }^{13} \mathrm{C}$ NMR $\left(101 \mathrm{MHz}, \mathrm{CDCl}_{3}, \delta\right): 55.8\left(\mathrm{CH}_{3}\right), 98.0-98.3(\mathrm{~m}, \mathrm{CH})$, $110.9\left(\mathrm{t}, J_{C-\mathrm{F}}=19.4 \mathrm{~Hz}, C\right), 126.1(\mathrm{CH}), 126.3(\mathrm{CH}), 126.7(\mathrm{C}), 127.6(\mathrm{CH}), 127.7(\mathrm{CH}), 128.0(\mathrm{CH})$, $128.1(\mathrm{CH}), 129.6(\mathrm{CH}), 132.6(C), 133.1(C), 160.1\left(\mathrm{t}, J_{C-\mathrm{F}}=14.0 \mathrm{~Hz}, C\right), 160.8\left(\mathrm{dd}, J_{C-\mathrm{F}}=10.4\right.$, $247.5 \mathrm{~Hz}, C) .{ }^{19} \mathrm{~F}$ NMR $\left(376 \mathrm{MHz}, \mathrm{CDCl}_{3}, \delta\right):-114.7$ (d, $\left.J=9.0 \mathrm{~Hz}, 2 \mathrm{~F}\right)$. HRMS-EI $(\mathrm{m} / \mathrm{z}):[\mathrm{M}]^{+}$calcd for $\mathrm{C}_{17} \mathrm{H}_{12} \mathrm{~F}_{2} \mathrm{O}_{1}, 270.0856$; found, 270.0858 .

\section{2-(2,6-Difluoro-3-methoxyphenyl)naphthalene (3ag).}

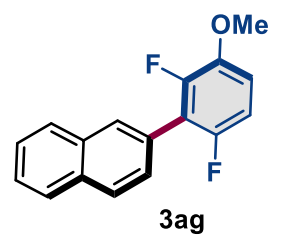

The reaction was carried out with $41.2 \mathrm{mg}(0.20 \mathrm{mmol})$ of $\mathbf{1 a a}$ and $56.6 \mathrm{mg}(0.30 \mathrm{mmol})$ of $\mathbf{2 h}$ according to Procedure E. The product 3ag was obtained as a yellow oil (34.3 mg, $0.13 \mathrm{mmol}, 64 \%$ yield) after purification by silica-gel column chromatography $\left(\mathrm{SiO}_{2}\right.$, EtOAc/hexane, 0:100-6:94). ${ }^{1} \mathrm{H}$ and ${ }^{13} \mathrm{C}$ NMR were in agreement with the literature. ${ }^{14}$

${ }^{1} \mathrm{H}$ NMR (400 MHz, $\mathrm{CDCl}_{3}, \delta$ ): 3.93 (s, 3H), 6.90-7.00 (m, 2H), 7.49-7.54 (m, 2H), 7.57 (dq, $J=1.6$, $8.4 \mathrm{~Hz}, 1 \mathrm{H}), 7.85-7.91(\mathrm{~m}, 2 \mathrm{H}), 7.93(\mathrm{~d}, J=8.4 \mathrm{~Hz}, 1 \mathrm{H}), 7.97$ (s, 1H). ${ }^{13} \mathrm{C} \mathrm{NMR}\left(101 \mathrm{MHz}, \mathrm{CDCl}_{3}\right.$,

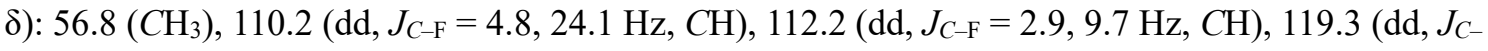
$\mathrm{F}=16.4,20.4 \mathrm{~Hz}, \mathrm{C}), 126.2(\mathrm{CH}), 126.5(\mathrm{CH}), 126.6(\mathrm{C}), 127.7(\mathrm{CH}), 127.8(\mathrm{CH}), 128.2(\mathrm{CH}), 129.8$ $(C \mathrm{H}), 132.9(C), 133.1(C), 144.6\left(\mathrm{dd}, J_{C-\mathrm{F}}=3.4,12.1 \mathrm{~Hz}, C\right), 149.6\left(\mathrm{dd}, J_{C-\mathrm{F}}=6.8,249.7 \mathrm{~Hz}, C\right)$, $153.8\left(\mathrm{dd}, J_{C-\mathrm{F}}=5.3,242.5 \mathrm{~Hz}, C\right) .{ }^{19} \mathrm{~F}$ NMR $\left(376 \mathrm{MHz}, \mathrm{CDCl}_{3}, \delta\right):-136.5(\mathrm{~d}, J=6.8 \mathrm{~Hz}, 1 \mathrm{~F}),-126.2-$ -126.1 (m, 1F). HRMS-EI $(\mathrm{m} / \mathrm{z})$ : [M] $]^{+}$calcd for $\mathrm{C}_{17} \mathrm{H}_{12} \mathrm{~F}_{2} \mathrm{O}_{1}, 270.0856$; found, 270.0854 . 


\section{2-(2-Ethoxy-6-fluorophenyl)naphthalene (3ah).}

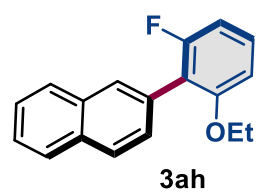

The reaction was carried out with $41.8 \mathrm{mg}(0.20 \mathrm{mmol})$ of $\mathbf{1 a a}$ and $55.2 \mathrm{mg}(0.30 \mathrm{mmol})$ of $\mathbf{2} \mathbf{i}$ according to Procedure E. The product 3ah was obtained as a yellow oil (44.8 $\mathrm{mg}, 0.17 \mathrm{mmol}, 83 \%$ yield) after purification by silica-gel column chromatography $\left(\mathrm{SiO}_{2}\right.$, EtOAc/hexane, 0:100-6:94).

${ }^{1} \mathrm{H}$ NMR (400 MHz, $\left.\mathrm{CDCl}_{3}, \delta\right): 1.28(\mathrm{t}, J=7.1 \mathrm{~Hz}, 3 \mathrm{H}), 4.03$ (q, $\left.J=7.1 \mathrm{~Hz}, 2 \mathrm{H}\right), 6.77-6.85(\mathrm{~m}, 2 \mathrm{H})$, 7.24-7.31 (m, 1H), 7.46-7.52 (m, 2H), 7.48-7.50 (m, 2H), 7.55 (dt, $J=1.4,8.7 \mathrm{~Hz}, 1 \mathrm{H}), 7.83-7.90$ $(\mathrm{m}, 3 \mathrm{H}), 7.91(\mathrm{~s}, 1 \mathrm{H}) .{ }^{13} \mathrm{C} \mathrm{NMR}\left(101 \mathrm{MHz}, \mathrm{CDCl}_{3}, \delta\right): 14.6\left(\mathrm{CH}_{3}\right), 64.5\left(\mathrm{CH}_{2}\right), 108.0\left(\mathrm{~d}, J_{C-\mathrm{F}}=2.9\right.$ $\mathrm{Hz}, C \mathrm{H}), 108.2\left(\mathrm{~d}, J_{C-\mathrm{F}}=24.2 \mathrm{~Hz}, C \mathrm{H}\right), 118.9\left(\mathrm{~d}, J_{C-\mathrm{F}}=17.5 \mathrm{~Hz}, C\right), 125.8(\mathrm{CH}), 125.9(C \mathrm{H}), 127.0$ $(\mathrm{CH}), 127.6(\mathrm{CH}), 128.1(\mathrm{CH}), 128.8(\mathrm{CH}), 128.9(\mathrm{CH}), 129.2(\mathrm{C}), 129.8(\mathrm{CH}), 132.6(\mathrm{C}), 133.1(\mathrm{C})$, $157.4\left(\mathrm{~d}, J_{C-\mathrm{F}}=6.8 \mathrm{~Hz}, C\right), 160.5\left(\mathrm{~d}, J_{C-\mathrm{F}}=245.8 \mathrm{~Hz}, C\right) .{ }^{19} \mathrm{~F} \mathrm{NMR}\left(376 \mathrm{MHz}, \mathrm{CDCl}_{3}, \delta\right): 116.5(\mathrm{t}, J$ $=7.5 \mathrm{~Hz}, 1 \mathrm{~F})$. HRMS-EI $(\mathrm{m} / \mathrm{z}):[\mathrm{M}]^{+}$calcd for $\mathrm{C}_{18} \mathrm{H}_{15} \mathrm{~F}_{1} \mathrm{O}_{1}, 266.1107$; found, 266.1104.

\section{2-(2-Chloro-6-fluorophenyl)naphthalene (3ai).}

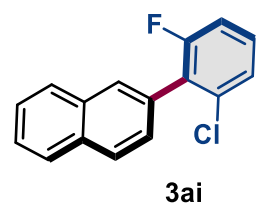

The reaction was carried out with $41.6 \mathrm{mg}(0.20 \mathrm{mmol})$ of $\mathbf{1 a a}$ and $52.7 \mathrm{mg}(0.30 \mathrm{mmol})$ of $\mathbf{2} \mathbf{j}$ according to Procedure E. The product 3ai was obtained as a white powder $(42.4 \mathrm{mg}, 0.17 \mathrm{mmol}$, $82 \%$ yield) after purification by silica-gel column chromatography $\left(\mathrm{SiO}_{2}\right.$, hexane only).

${ }^{1} \mathrm{H}$ NMR (400 MHz, $\left.\mathrm{CDCl}_{3}, \delta\right): 7.09-7.15(\mathrm{~m}, 1 \mathrm{H}), 7.27-7.35$ (m, 2H), $7.51(\mathrm{~d}, J=27.6 \mathrm{~Hz}, 1 \mathrm{H})$, 7.49-7.56 (m, 3H), 7.84-7.92 (m, 3H), 7.93 (d, $\left.J=8.8 \mathrm{~Hz}, 1 \mathrm{H}) .{ }^{13} \mathrm{C} \mathrm{NMR} \mathrm{(101} \mathrm{MHz,} \mathrm{CDCl}_{3}, \delta\right)$ : $114.3\left(\mathrm{~d}, J_{C-\mathrm{F}}=23.2 \mathrm{~Hz}, \mathrm{CH}\right), 125.5\left(\mathrm{~d}, J_{C-\mathrm{F}}=3.8 \mathrm{~Hz}, \mathrm{CH}\right), 126.2(\mathrm{CH}), 126.5(\mathrm{CH}), 127.7(\mathrm{CH})$, $127.8(C \mathrm{H}), 128.2(\mathrm{CH}), 129.0\left(\mathrm{~d}, J_{C-\mathrm{F}}=19.3 \mathrm{~Hz}, C\right), 129.2\left(\mathrm{~d}, J_{C-\mathrm{F}}=9.7 \mathrm{~Hz}, C\right), 129.5(C \mathrm{H}), 130.0$ (C), $132.9(C), 133.0(C), 134.6\left(\mathrm{~d}, J_{C-\mathrm{F}}=3.9 \mathrm{~Hz}, C\right), 160.4\left(\mathrm{~d}, J_{C-\mathrm{F}}=249.7 \mathrm{~Hz}, C\right) .{ }^{19} \mathrm{~F}$ NMR $(376$ $\left.\mathrm{MHz}, \mathrm{CDCl}_{3}, \delta\right):-112.4(\mathrm{t}, J=7.1 \mathrm{~Hz}, 1 \mathrm{~F})$. HRMS-EI $(\mathrm{m} / \mathrm{z})$ : $[\mathrm{M}]^{+}$calcd for $\mathrm{C}_{16} \mathrm{H}_{10} \mathrm{Cl}_{1} \mathrm{~F}_{1}, 256.0455$; found, 256.0451 . 


\section{2-(2,6-Dichlorophenyl)naphthalene (3aj).}

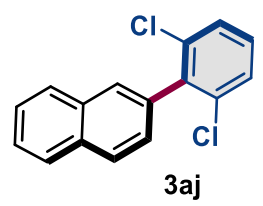

The reaction was carried out with $41.9 \mathrm{mg}(0.20 \mathrm{mmol})$ of $\mathbf{1}$ aa and $57.9 \mathrm{mg}(0.30 \mathrm{mmol})$ of $\mathbf{2 k}$ according to Procedure E. The product 3aj was obtained as a colorless oil (51.8 mg, $0.19 \mathrm{mmol}, 94 \%$ yield) after purification by silica-gel column chromatography $\left(\mathrm{SiO}_{2}\right.$, hexane only).

${ }^{1} \mathrm{H}$ NMR $\left(400 \mathrm{MHz}, \mathrm{CDCl}_{3}, \delta\right): 7.23-7.29(\mathrm{~m}, 1 \mathrm{H}), 7.38(\mathrm{dd}, J=1.6,8.4 \mathrm{~Hz}, 1 \mathrm{H}), 7.44(\mathrm{~d}, J=8.0 \mathrm{~Hz}$, 2H), 7.50-7.56 (m, 2H), $7.76(\mathrm{~s}, 1 \mathrm{H}), 7.85-7.97(\mathrm{~m}, 3 \mathrm{H}) .{ }^{13} \mathrm{C} \mathrm{NMR}\left(101 \mathrm{MHz}, \mathrm{CDCl}_{3}, \delta\right): 126.2(\mathrm{CH})$, $126.4(\mathrm{CH}), 127.3(\mathrm{CH}), 127.8(\mathrm{CH}), 128.1(\mathrm{CH}), 128.2(\mathrm{CH}), 128.8(\mathrm{CH}), 129.1(\mathrm{CH}), 132.8(\mathrm{C})$, 133.0 (C), $134.4(C), 135.1(C), 139.4(C)$. HRMS-EI $(\mathrm{m} / \mathrm{z})$ : [M] $]^{+}$calcd for $\mathrm{C}_{16} \mathrm{H}_{10} \mathrm{Cl}_{2}, 272.0160$; found, 272.0157

\section{2-(Naphthalen-2-yl)thiophene (3ak).}

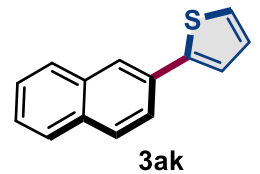

The reaction was carried out with $41.9 \mathrm{mg}(0.20 \mathrm{mmol})$ of $\mathbf{1 a a}$ and $39.0 \mathrm{mg}(0.30 \mathrm{mmol})$ of $\mathbf{2 l}$ according to Procedure E. The product 3ak was obtained as a white powder $(41.0 \mathrm{mg}, 0.19 \mathrm{mmol}$, $96 \%$ yield) after purification by silica-gel column chromatography $\left(\mathrm{SiO}_{2}\right.$, hexane only). ${ }^{1} \mathrm{H}$ and ${ }^{13} \mathrm{C}$ NMR were in agreement with the literature. ${ }^{15}$

${ }^{1} \mathrm{H}$ NMR (400 MHz, $\left.\mathrm{CDCl}_{3}, \delta\right): 7.13$ (dd, $\left.J=3.4,5.0 \mathrm{~Hz}, 1 \mathrm{H}\right), 7.33$ (dd, $\left.J=1.2,5.2 \mathrm{~Hz}, 1 \mathrm{H}\right), 7.43-$ $7.52(\mathrm{~m}, 3 \mathrm{H}), 7.76(\mathrm{dd}, J=1.6,8.4 \mathrm{~Hz}, 1 \mathrm{H}), 7.80-7.88(\mathrm{~m}, 3 \mathrm{H}), 8.06(\mathrm{~s}, 1 \mathrm{H}) .{ }^{13} \mathrm{C}$ NMR $(101 \mathrm{MHz}$, $\left.\mathrm{CDCl}_{3}, \delta\right): 123.5(\mathrm{CH}), 124.3(\mathrm{CH}), 124.5(\mathrm{CH}), 125.0(\mathrm{CH}), 125.9(\mathrm{CH}), 126.5(\mathrm{CH}), 127.7(\mathrm{CH})$, $128.0(\mathrm{CH}), 128.1(\mathrm{CH}), 128.5(\mathrm{CH}), 131.9(\mathrm{C}), 132.8(\mathrm{C}), 133.7(\mathrm{C}), 144.5(\mathrm{C})$. HRMS-EI $(\mathrm{m} / \mathrm{z})$ : $[\mathrm{M}]^{+}$calcd for $\mathrm{C}_{14} \mathrm{H}_{10} \mathrm{~S}_{1}, 210.0503$; found, 210.0499 .

\section{2-(Naphthalen-2-yl)furan (3al).}

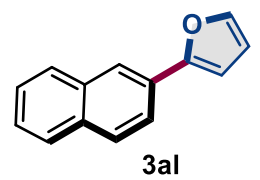

The reaction was carried out with $41.6 \mathrm{mg}(0.20 \mathrm{mmol})$ of $\mathbf{1}$ aa and $34.0 \mathrm{mg}(0.30 \mathrm{mmol})$ of $\mathbf{2 m}$ according to Procedure E. The product 3al was obtained as a white powder $(36.0 \mathrm{mg}, 0.19 \mathrm{mmol}$, $92 \%$ yield) after purification by silica-gel column chromatography $\left(\mathrm{SiO}_{2}\right.$, hexane only). ${ }^{1} \mathrm{H}$ and ${ }^{13} \mathrm{C}$ NMR were in agreement with the literature. ${ }^{15}$ 
${ }^{1} \mathrm{H} \mathrm{NMR}\left(396 \mathrm{MHz}, \mathrm{CDCl}_{3}, \delta\right): 6.53(\mathrm{dd}, J=1.6,3.2 \mathrm{~Hz}, 1 \mathrm{H}), 6.78(\mathrm{~d}, J=3.2 \mathrm{~Hz}, 1 \mathrm{H}), 7.47$ (quintd, $J=1.4,7.4 \mathrm{~Hz}, 2 \mathrm{H}), 7.53(\mathrm{~d}, J=2.0 \mathrm{~Hz}, 1 \mathrm{H}), 7.75-7.89(\mathrm{~m}, 4 \mathrm{H}), 8.15(\mathrm{~s}, 1 \mathrm{H}) .{ }^{13} \mathrm{C} \mathrm{NMR}(100 \mathrm{MHz}$, $\left.\mathrm{CDCl}_{3}, \delta\right): 105.6(\mathrm{CH}), 111.8(\mathrm{CH}), 122.1(\mathrm{CH}), 122.3(\mathrm{CH}), 125.9(\mathrm{CH}), 126.4(\mathrm{CH}), 127.7(\mathrm{CH})$, $128.1(\mathrm{CH}), 128.3(\mathrm{CH}), 132.6(\mathrm{C}), 133.5(\mathrm{C}), 142.3(\mathrm{CH}), 154.0(\mathrm{C})$. HRMS-EI $(\mathrm{m} / \mathrm{z})$ : $[\mathrm{M}]^{+}$calcd for $\mathrm{C}_{14} \mathrm{H}_{10} \mathrm{O}_{1}, 194.0732$; found, 194.0729 .

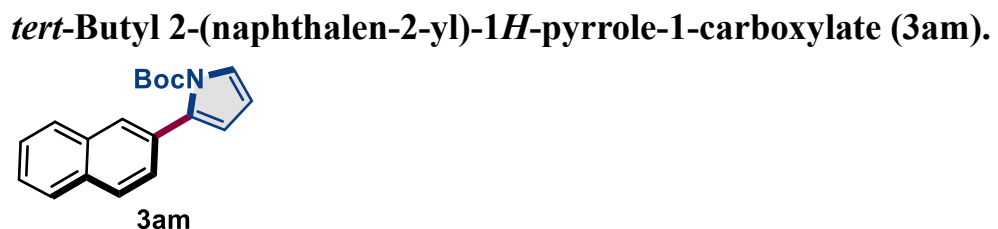

The reaction was carried out with $41.3 \mathrm{mg}(0.20 \mathrm{mmol})$ of 1 aa and $63.9 \mathrm{mg}(0.30 \mathrm{mmol})$ of $\mathbf{2 n}$ according to Procedure E. The product 3am was obtained as a white powder $(50.2 \mathrm{mg}, 0.17 \mathrm{mmol}$, $86 \%$ yield) after purification by silica-gel column chromatography $\left(\mathrm{SiO}_{2}, \mathrm{Et}_{2} \mathrm{O} /\right.$ hexane, 0:100-4:96) and GPC.

${ }^{1} \mathrm{H}$ NMR (396 MHz, $\left.\mathrm{CDCl}_{3}, \delta\right): 1.32$ (s, 9H), 6.25-6.31 (m, 2H), 7.40 (dd, $\left.J=1.6,3.2 \mathrm{~Hz}, 1 \mathrm{H}\right), 7.44-$ $7.50(\mathrm{~m}, 3 \mathrm{H}), 7.78-7.86(\mathrm{~m}, 4 \mathrm{H}) .{ }^{13} \mathrm{C} \mathrm{NMR}\left(100 \mathrm{MHz}, \mathrm{CDCl}_{3}, \delta\right): 27.5\left(\mathrm{CH}_{3}\right), 83.6(\mathrm{C}), 110.7(\mathrm{CH})$, 114.8 $(\mathrm{CH}), 122.7(\mathrm{CH}), 125.8(\mathrm{CH}), 126.0(\mathrm{CH}), 126.7(\mathrm{CH}), 127.2(\mathrm{CH}), 127.6(\mathrm{CH}), 127.8(\mathrm{CH})$, $127.9(\mathrm{CH}), 131.8(\mathrm{C}), 132.4(\mathrm{C}), 132.9(\mathrm{C}), 135.0(\mathrm{C}), 149.3(\mathrm{C})$. HRMS-EI $(\mathrm{m} / \mathrm{z})$ : [M] $]^{+}$calcd for $\mathrm{C}_{19} \mathrm{H}_{1}{ }_{9} \mathrm{~N}_{1} \mathrm{O}_{2}, 293.1416$; found, 293.1423 .

\section{2-(Naphthalen-2-yl)benzo[b]thiophene (3an).}

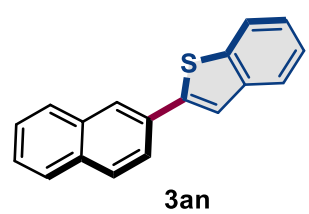

The reaction was carried out with $41.1 \mathrm{mg}(0.20 \mathrm{mmol})$ of 1 aa and $54.3 \mathrm{mg}(0.30 \mathrm{mmol})$ of $2 \mathrm{o}$ according to Procedure E. The product 3an was obtained as a white powder $(53.5 \mathrm{mg}, 0.20 \mathrm{mmol}$, $99 \%$ yield) after purification by silica-gel column chromatography $\left(\mathrm{SiO}_{2}, \mathrm{CH}_{2} \mathrm{Cl}_{2} /\right.$ hexane, 0:100-25:75). ${ }^{1} \mathrm{H}$ and ${ }^{13} \mathrm{C}$ NMR were in agreement with the literature. ${ }^{16}$

${ }^{1} \mathrm{H}$ NMR (396 MHz, $\mathrm{CDCl}_{3}, \delta$ ): 7.31-7.40 (m, 2H), 7.47-7.56 (m, 2H), 7.69 (s, 1H), 7.76-7.92 (m, $6 \mathrm{H}), 8.16(\mathrm{~s}, 1 \mathrm{H}) .{ }^{13} \mathrm{C} \mathrm{NMR}\left(100 \mathrm{MHz}, \mathrm{CDCl}_{3}, \delta\right): 120.0(\mathrm{CH}), 122.3(\mathrm{CH}), 123.6(\mathrm{CH}), 124.5(\mathrm{CH})$, 124.6 (CH), $125.4(\mathrm{CH}), 126.4(\mathrm{CH}), 126.7(\mathrm{CH}), 127.8(\mathrm{CH}), 128.2(\mathrm{CH}), 128.6(\mathrm{CH}), 131.8(\mathrm{C})$, $133.2(C), 133.7(C), 139.7(C), 140.9(C), 144.4(C)$. HRMS-EI $(m / z)$ : $[\mathrm{M}]^{+}$calcd for $\mathrm{C}_{18} \mathrm{H}_{12} \mathrm{~S}_{1}$, 260.0660; found, 260.0658 . 


\section{2-(Naphthalen-2-yl)benzofuran (3ao).}

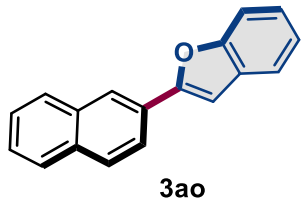

The reaction was carried out with $41.3 \mathrm{mg}(0.20 \mathrm{mmol})$ of $\mathbf{1} \mathbf{a a}$ and $48.5 \mathrm{mg}(0.30 \mathrm{mmol})$ of $\mathbf{2 p}$ according to Procedure E. The product 3ao was obtained as a white powder $(44.4 \mathrm{mg}, 0.18 \mathrm{mmol}$, $91 \%$ yield) after purification by silica-gel column chromatography $\left(\mathrm{SiO}_{2}, \mathrm{CH}_{2} \mathrm{Cl}_{2} /\right.$ hexane, 0:100-20:80). ${ }^{1} \mathrm{H}$ and ${ }^{13} \mathrm{C}$ NMR were in agreement with the literature. ${ }^{15}$

${ }^{1} \mathrm{H}$ NMR (396 MHz, $\left.\mathrm{CDCl}_{3}, \delta\right): 7.16$ (s, 1H), 7.23-7.34 (m, 1H), 7.32 (td, $\left.J=1.3,7.7 \mathrm{~Hz}, 1 \mathrm{H}\right), 7.47-$ $7.55(\mathrm{~m}, 2 \mathrm{H}), 7.57$ (d, $J=8.3 \mathrm{~Hz}, 1 \mathrm{H}), 7.62$ (d, $J=6.7 \mathrm{~Hz}, 1 \mathrm{H}), 7.82-7.88(\mathrm{~m}, 1 \mathrm{H}), 7.89-7.96(\mathrm{~m}$, 3H), 8.39 (s, $1 \mathrm{H}) .{ }^{13} \mathrm{C}$ NMR (100 MHz, $\left.\mathrm{CDCl}_{3}, \delta\right): 101.9(\mathrm{CH}), 111.1(\mathrm{CH}), 120.9(\mathrm{CH}), 122.8(\mathrm{CH})$, $123.0(\mathrm{CH}), 123.8(\mathrm{CH}), 124.4(\mathrm{CH}), 126.4(\mathrm{CH}), 126.6(\mathrm{CH}), 127.7(\mathrm{C}), 127.8(\mathrm{CH}), 128.4(\mathrm{CH})$, $128.5(\mathrm{CH}), 129.3(\mathrm{C}), 133.2(\mathrm{C}), 133.4(\mathrm{C}), 155.0(\mathrm{C}), 155.9(\mathrm{C})$. HRMS-EI $(\mathrm{m} / \mathrm{z})$ : $[\mathrm{M}]^{+}$calcd for $\mathrm{C}_{18} \mathrm{H}_{12} \mathrm{O}_{1}, 244.0888$; found, 244.0886. 


\section{References}

(1) Chen, L.; Francis, H.; Carrow, B. P. An "on-Cycle" Precatalyst Enables Room-Temperature Polyfluoroarylation Using Sensitive Boronic Acids. ACS Catal. 2018, 8, 2989-2994.

(2) Bruno, N. C.; Tudge, M. T.; Buchwald, S. L. Design and Preparation of New Palladium Precatalysts for C-C and C-N Cross-Coupling Reactions. Chem. Sci. 2013, 4, 916-920.

(3) Sheldrick, G. M. Crystal Structure Refinement with SHELXL. Acta Cryst. Sect. C, 2015, 71, 38.

(4) Lafrance, M.; Rowley, C. N.; Woo, T. K.; Fagnou, K. Catalytic Intermolecular Direct Arylation of Perfluorobenzenes. J. Am. Chem. Soc. 2006, 128, 8754-8756.

(5) Sheldon, R. A. The E Factor: Fifteen Years On. Green Chem. 2007, 9, 1273-1283.

(6) Sheldon, R. A. E Factors, Green Chemistry and Catalysis: An Odyssey. Chem. Commun. 2008, 3352-3365.

(7) Shang, R.; Xu, Q.; Jiang, Y. Y.; Wang, Y.; Liu, L. Pd-Catalyzed Decarboxylative Cross Coupling of Potassium Polyfluorobenzoates with Aryl Bromides, Chlorides, and Triflates. Org. Lett. 2010, $12,1000-1003$.

(8) Wei, Y.; Kan, J.; Wang, M.; Su, W.; Hong, M. Palladium-Catalyzed Direct Arylation of ElectronDeficient Polyfluoroarenes with Arylboronic Acids. Org. Lett. 2009, 11, 3346-3349.

(9) Yuen, O. Y.; Charoensak, M.; So, C. M.; Kuhakarn, C.; Kwong, F. Y. A General Direct Arylation of Polyfluoroarenes with Heteroaryl and Aryl Chlorides Catalyzed by Palladium Indolylphosphine Complexes. Chem. Asian J. 2015, 10, 857-861.

(10)Ma, X.; Gu, N.; Liu, Y.; Liu, P.; Xie, J.; Dai, B.; Liu, Z. One-Pot Synthesis of Polyfluoroterphenyls via Palladium-Catalyzed Suzuki-Miyaura Coupling of Chlorobromobenzene and $\mathrm{C}-\mathrm{H}$ Bond Functionalization of Perfluoroarenes. Appl. Organometal. Chem. 2015, 29, 50-56.

(11)He, C. Y.; Fan, S.; Zhang, X. Pd-Catalyzed Oxidative Cross-Coupling of Perfluoroarenes with Aromatic Heterocycles. J. Am. Chem. Soc. 2010, 132, 12850-12852.

(12)Chen, Q.; Wu, A.; Qin, S.; Zeng, M.; Le, Z.; Yan, Z.; Zhang, H. Ni-Catalyzed Decarboxylative Cross-Coupling of Potassium Polyfluorobenzoates with Unactivated Phenol and Phenylmethanol Derivatives. Adv. Synth. Catal. 2018, 360, 3239-3244.

(13)Daley, R. A.; Liu, E. C.; Topczewski, J. J. Additive-Free Palladium-Catalyzed Decarboxylative Cross-Coupling of Aryl Chlorides. Org. Lett. 2019, 21, 4734-4738.

(14)Yan, T.; Bheeter, C. B.; Doucet, H. Influence of 1,3-Difluorobenzene Substituents for PalladiumCatalyzed Direct Arylations. Eur. J. Org. Chem. 2013, 7152-7163.

(15)Vila, C.; Hornillos, V.; Giannerini, M.; Fañanás-Mastral, M.; Feringa, B. L. Palladium-Catalysed Direct Cross-Coupling of Organolithium Reagents with Aryl and Vinyl Triflates. Chem. Eur. J. 2014, 20, 13078-13083. 
(16)Cai, T.; Feng, C.; Shen, F.; Bian, K.; Wu, C.; Shen, R.; Gao, Y. Synthesis of 2-Substituted Benzothio(seleno)phenes and Indoles via Ag-Catalyzed Cyclization/Demethylation of 2Alkynylthio(seleno)anisoles and 2-Alkynyldimethylanilines. Eur. J. Org. Chem. 2021, 653-656. 


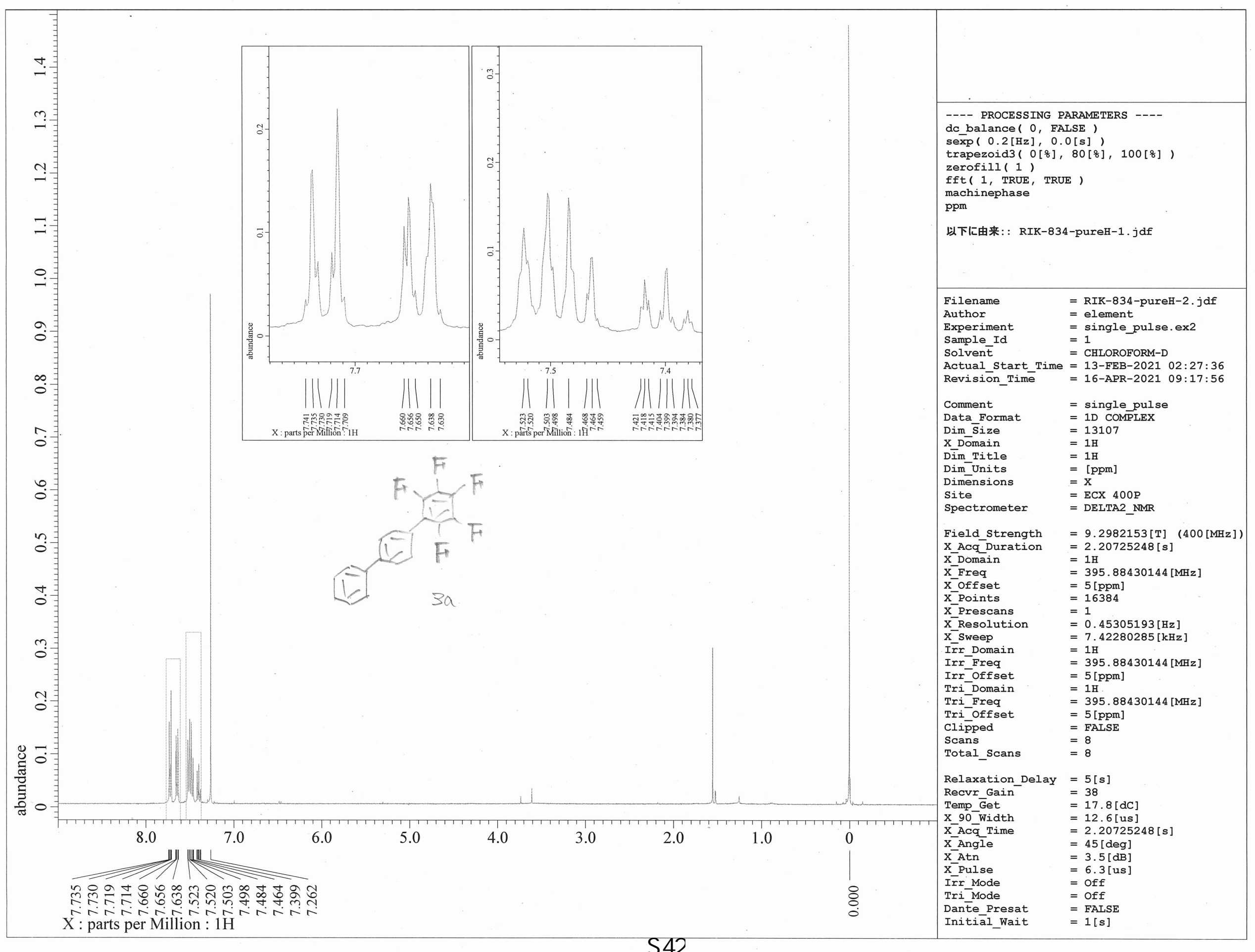




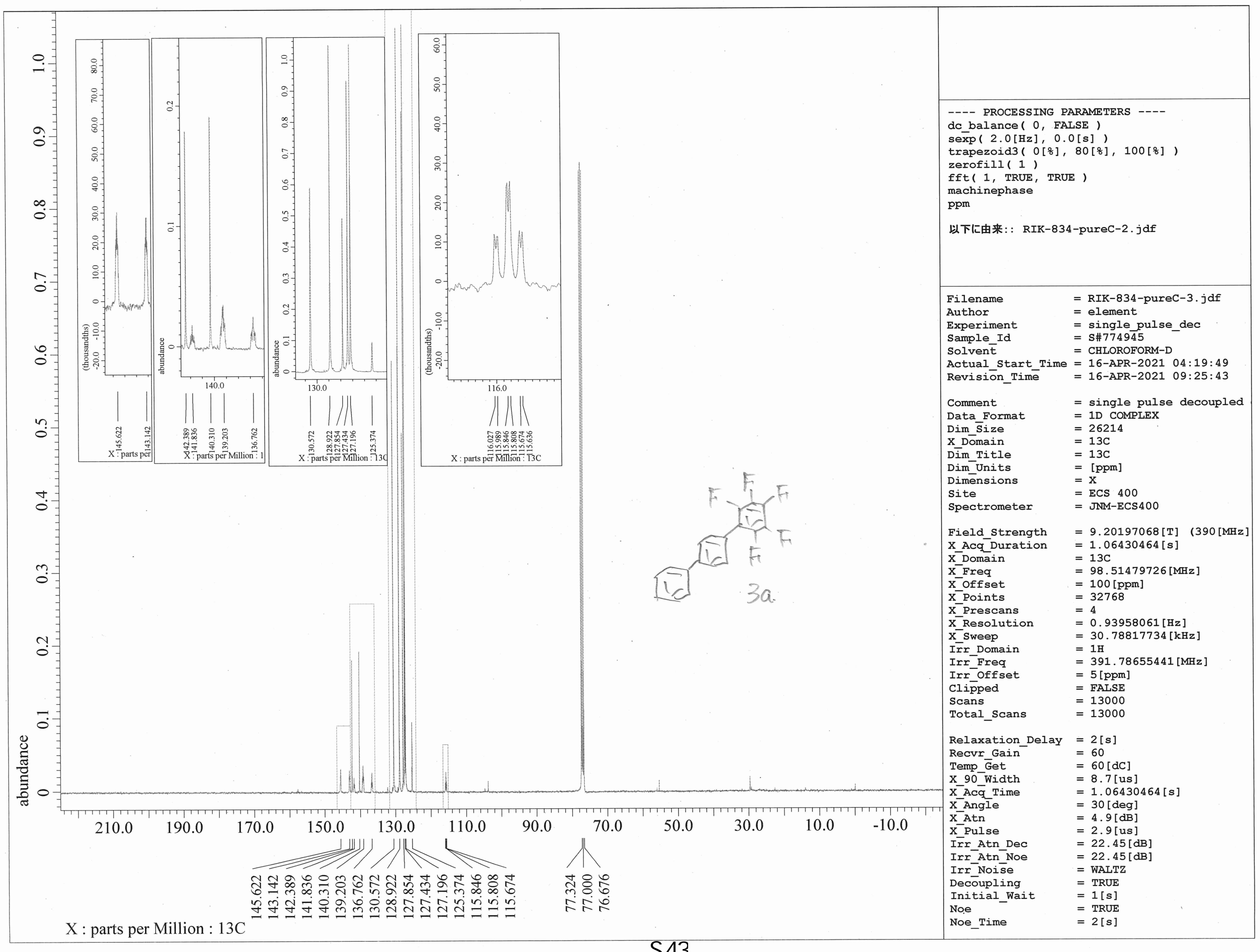




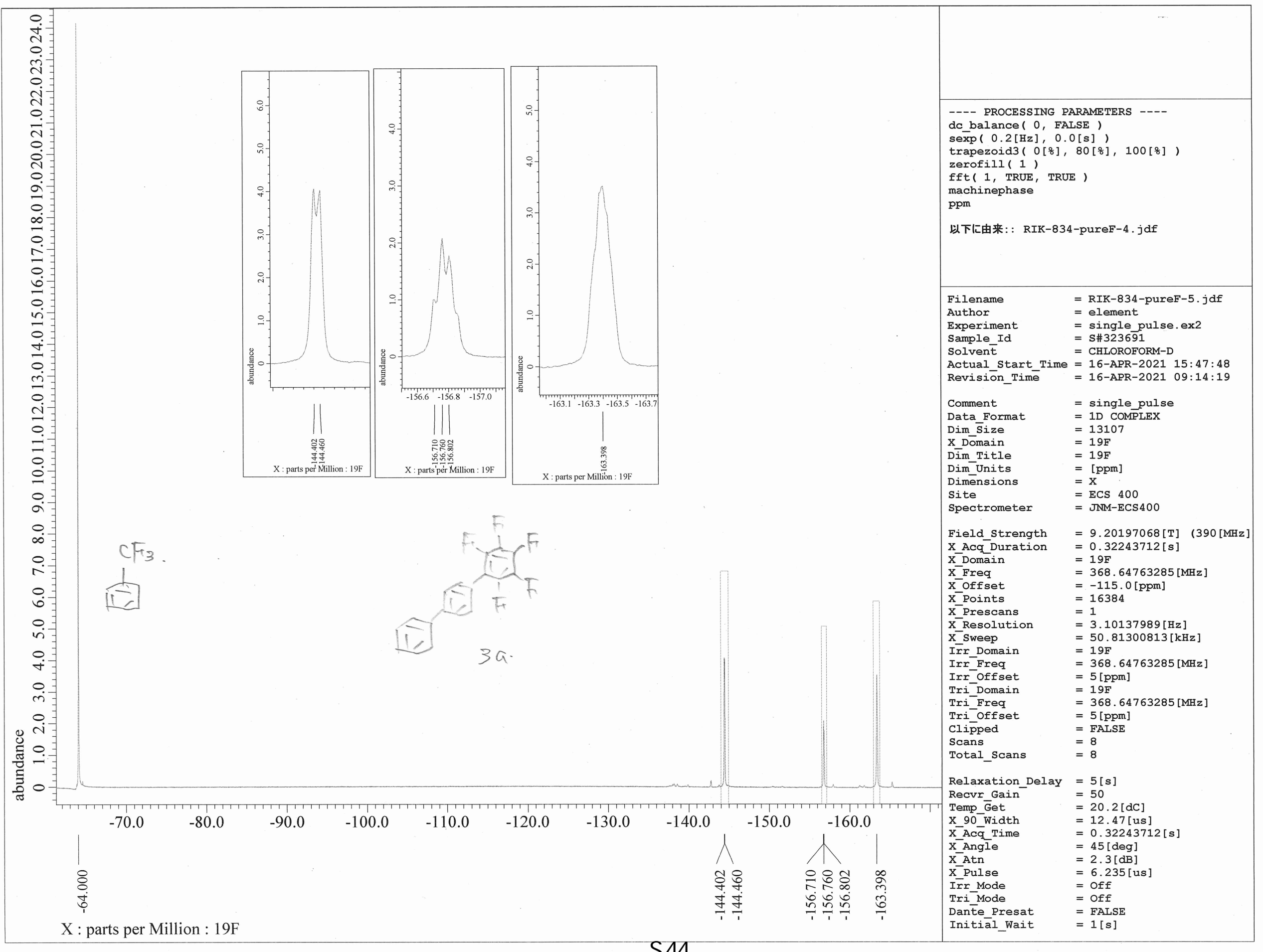




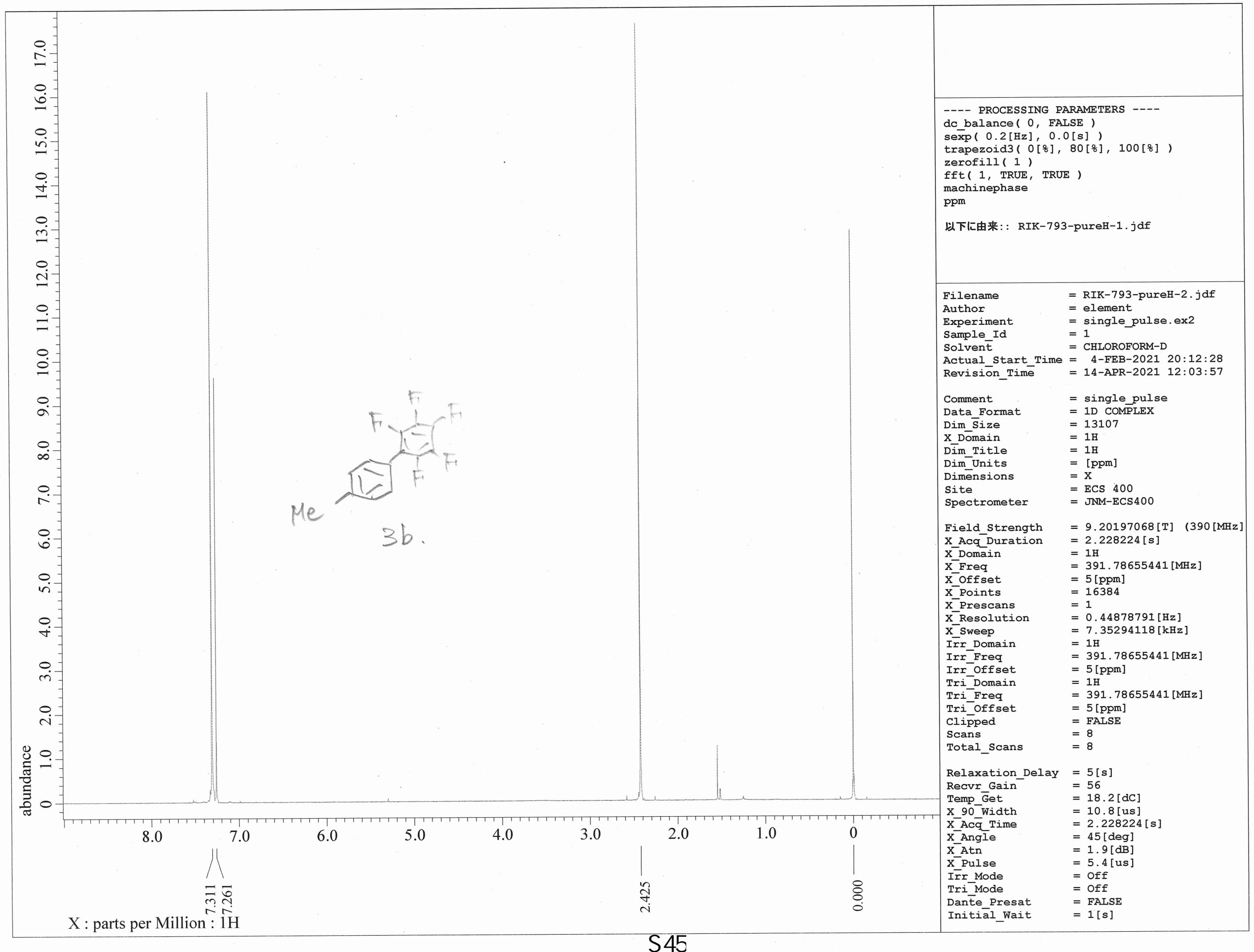




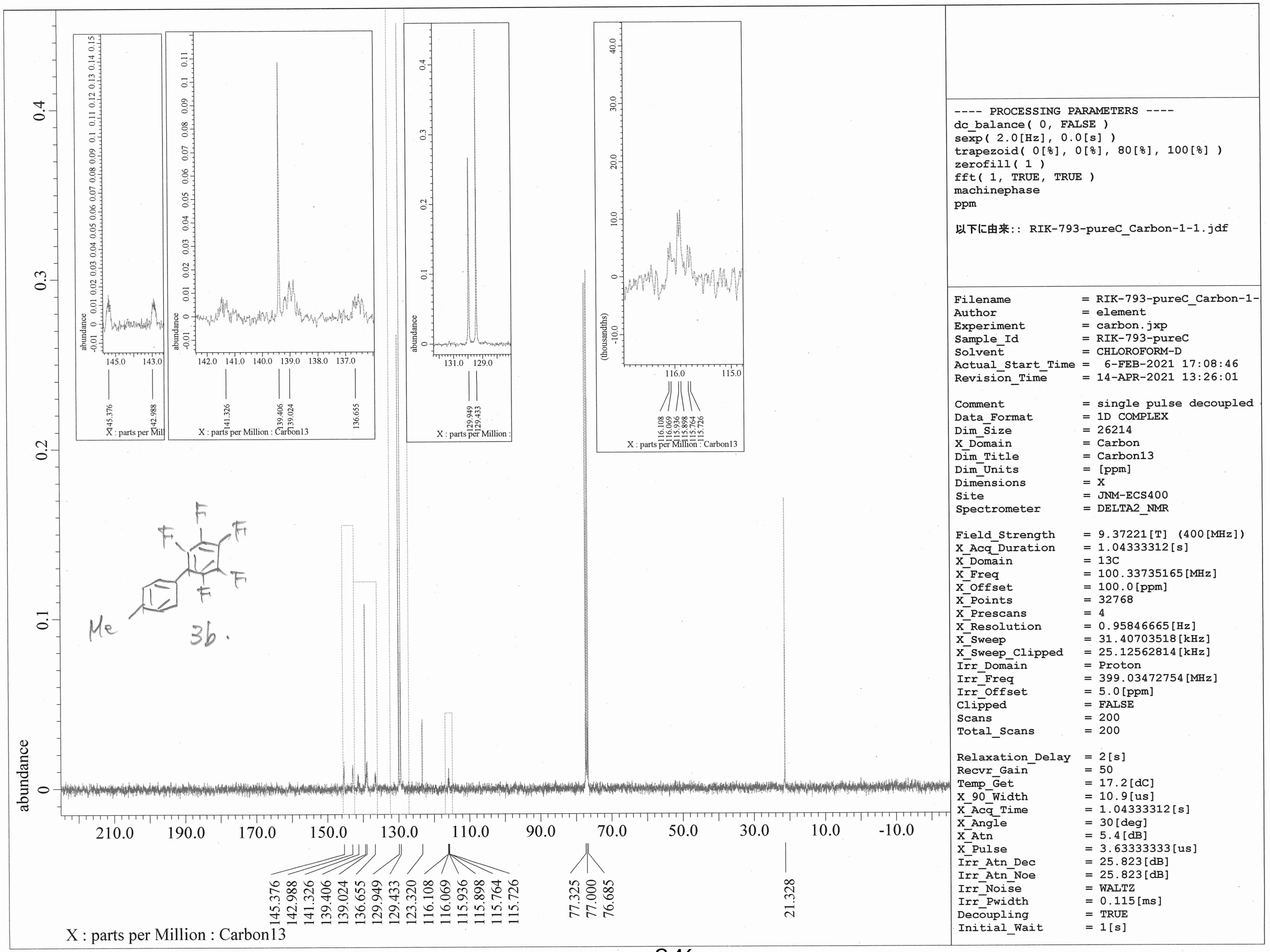




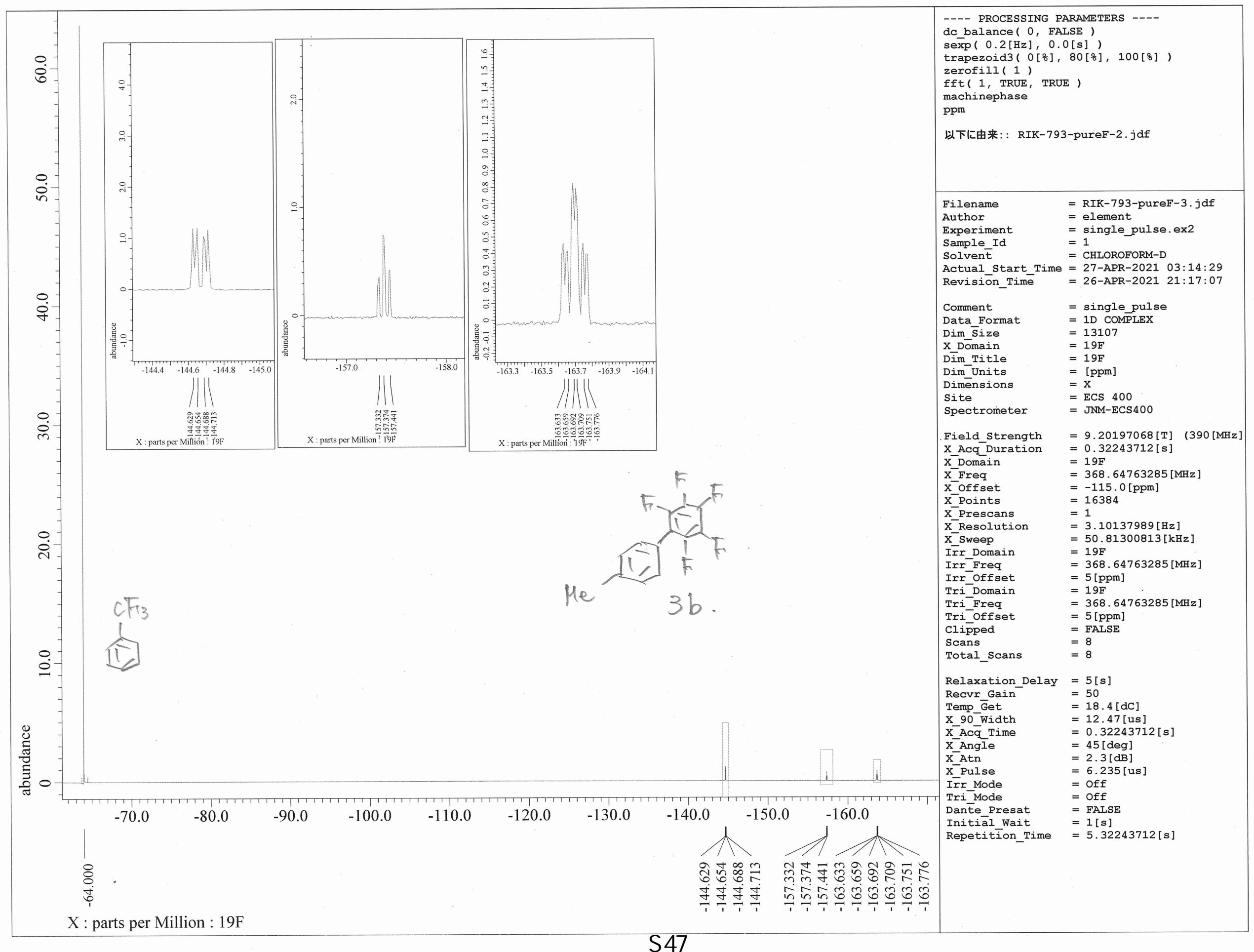




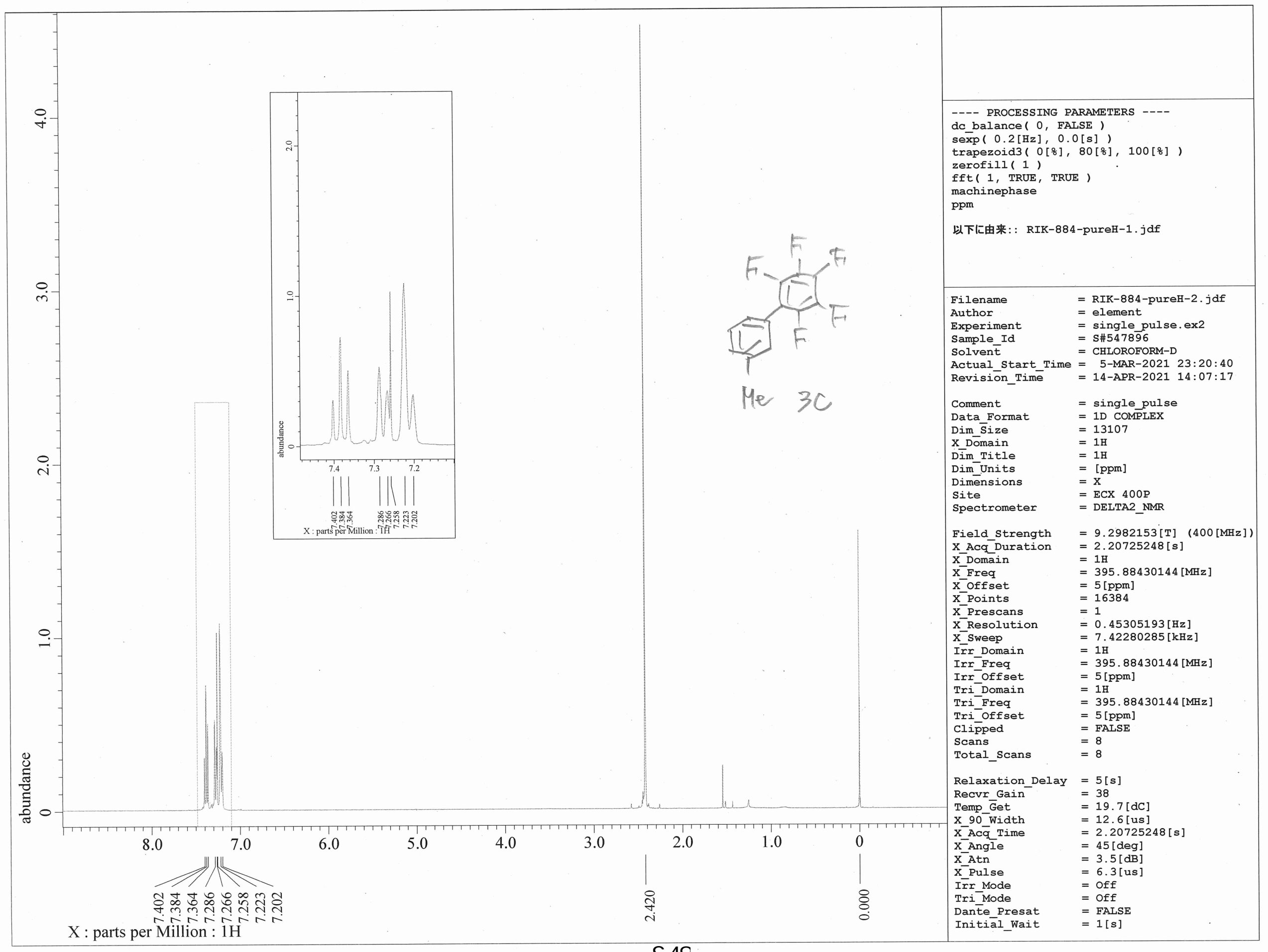




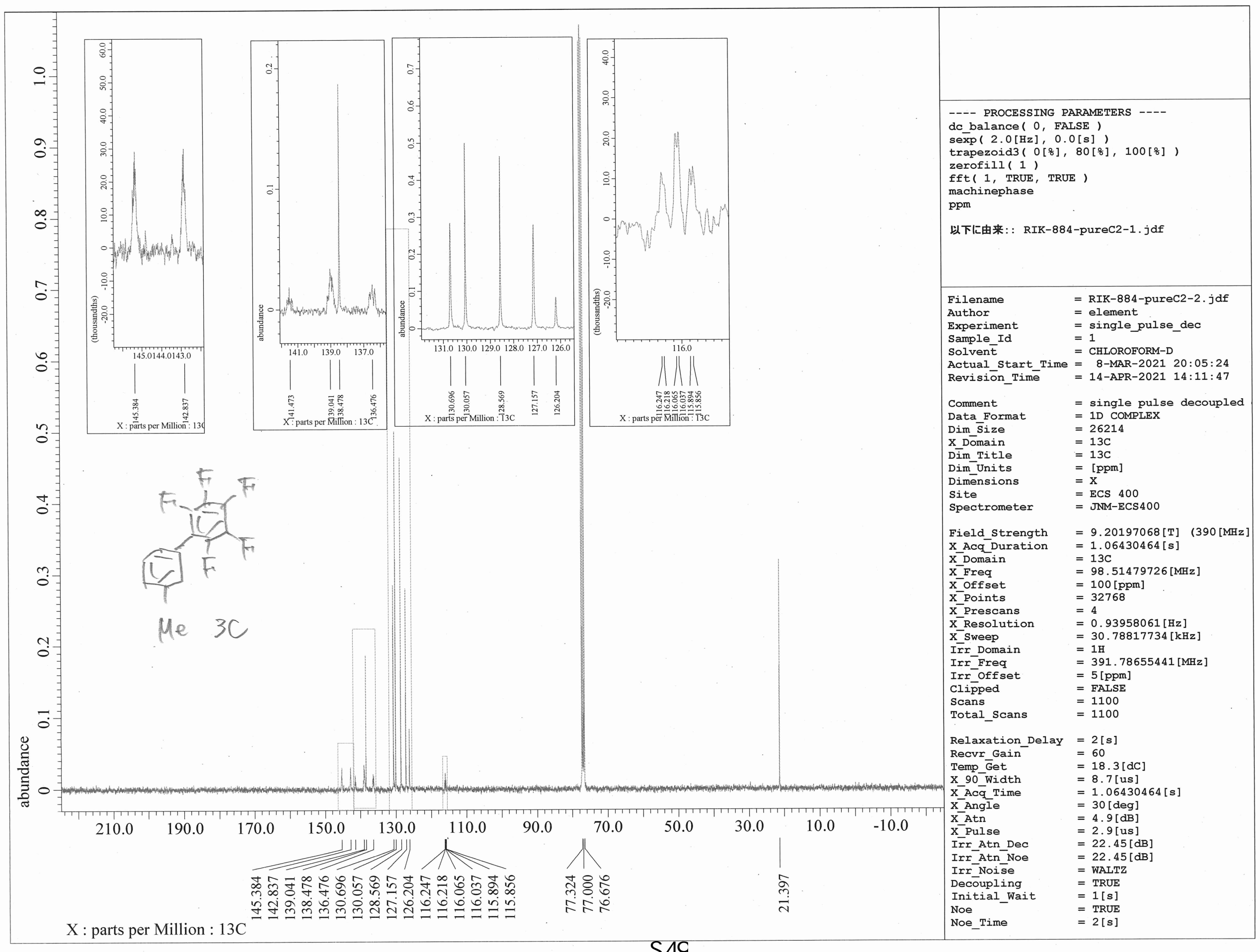




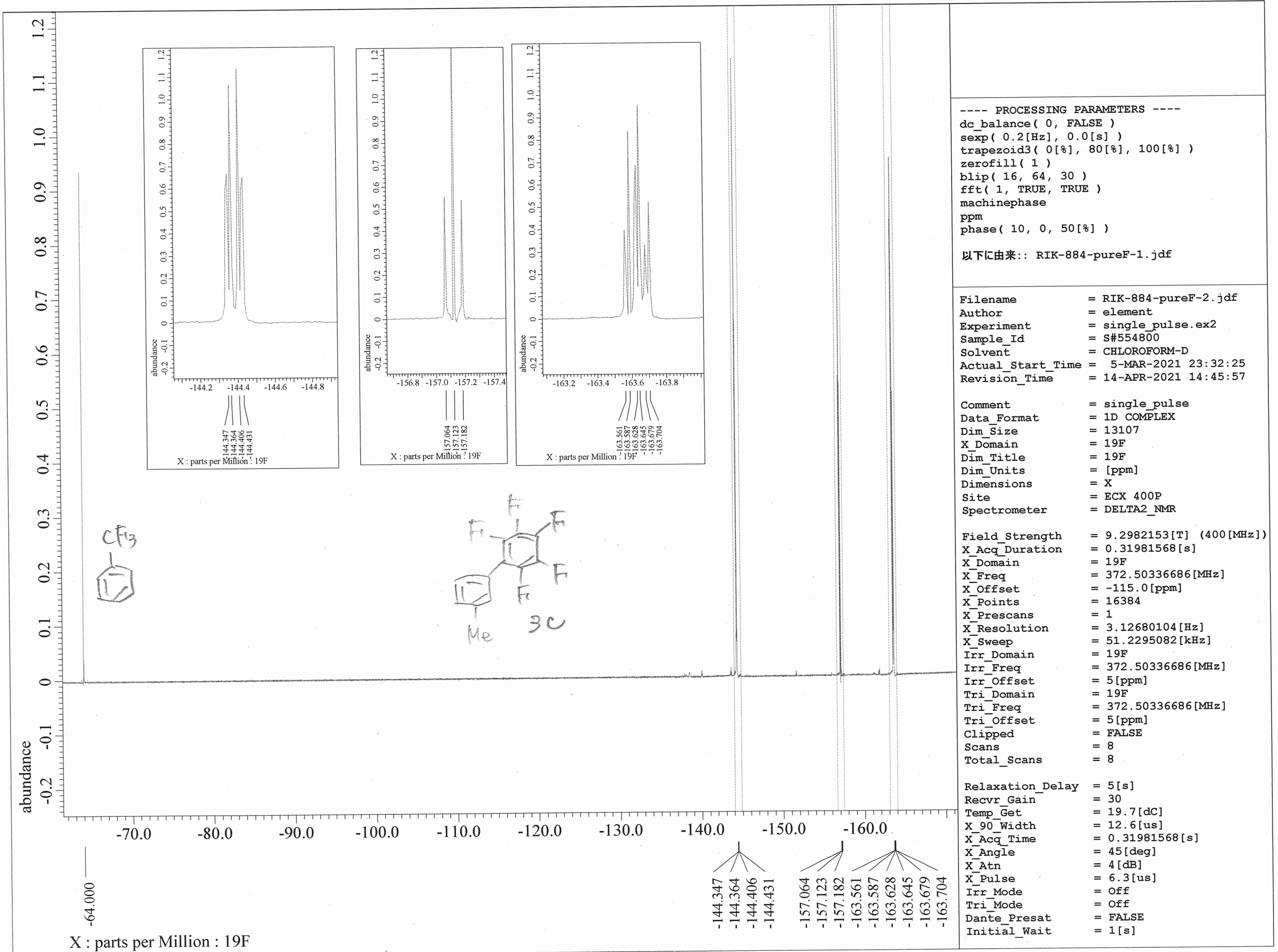




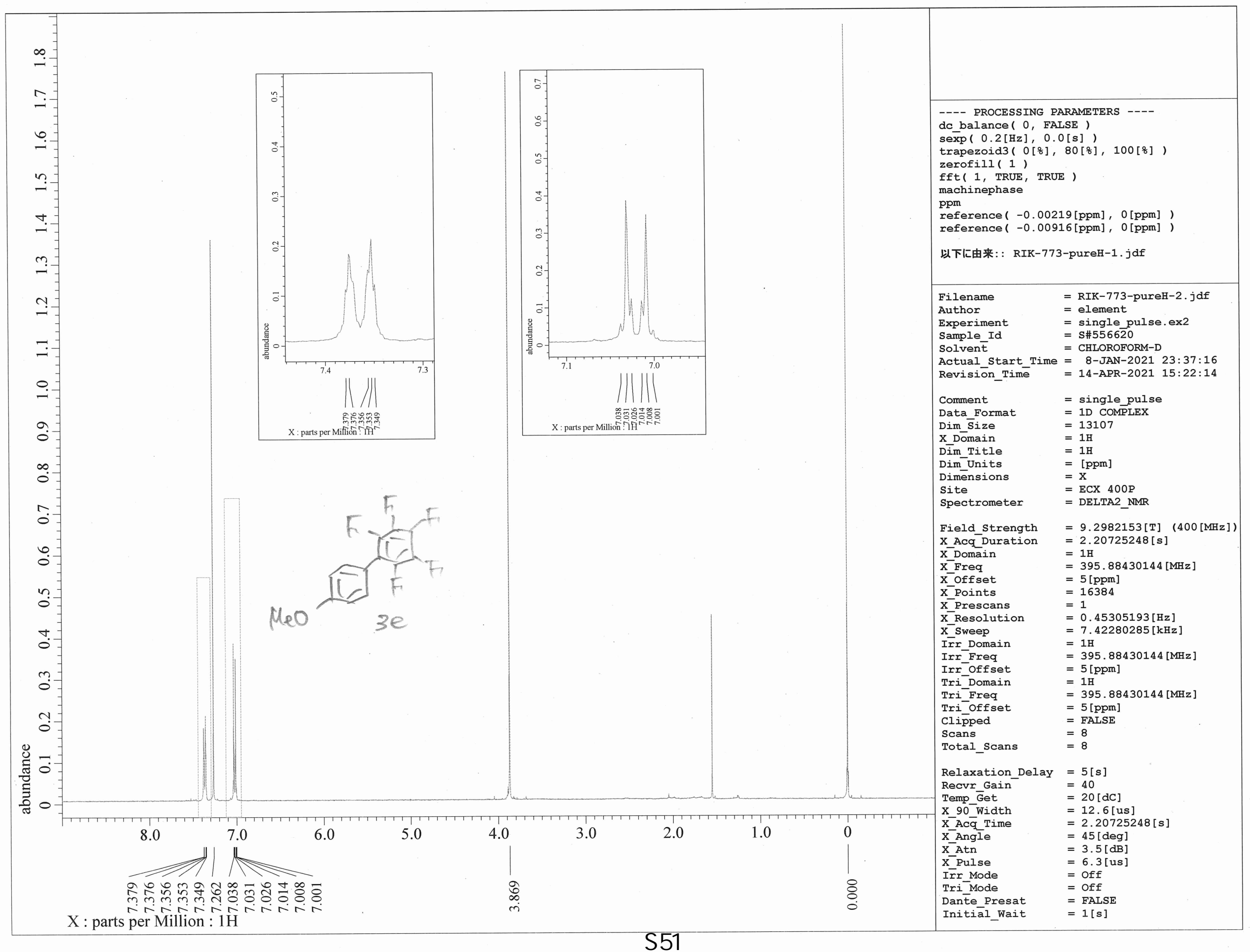




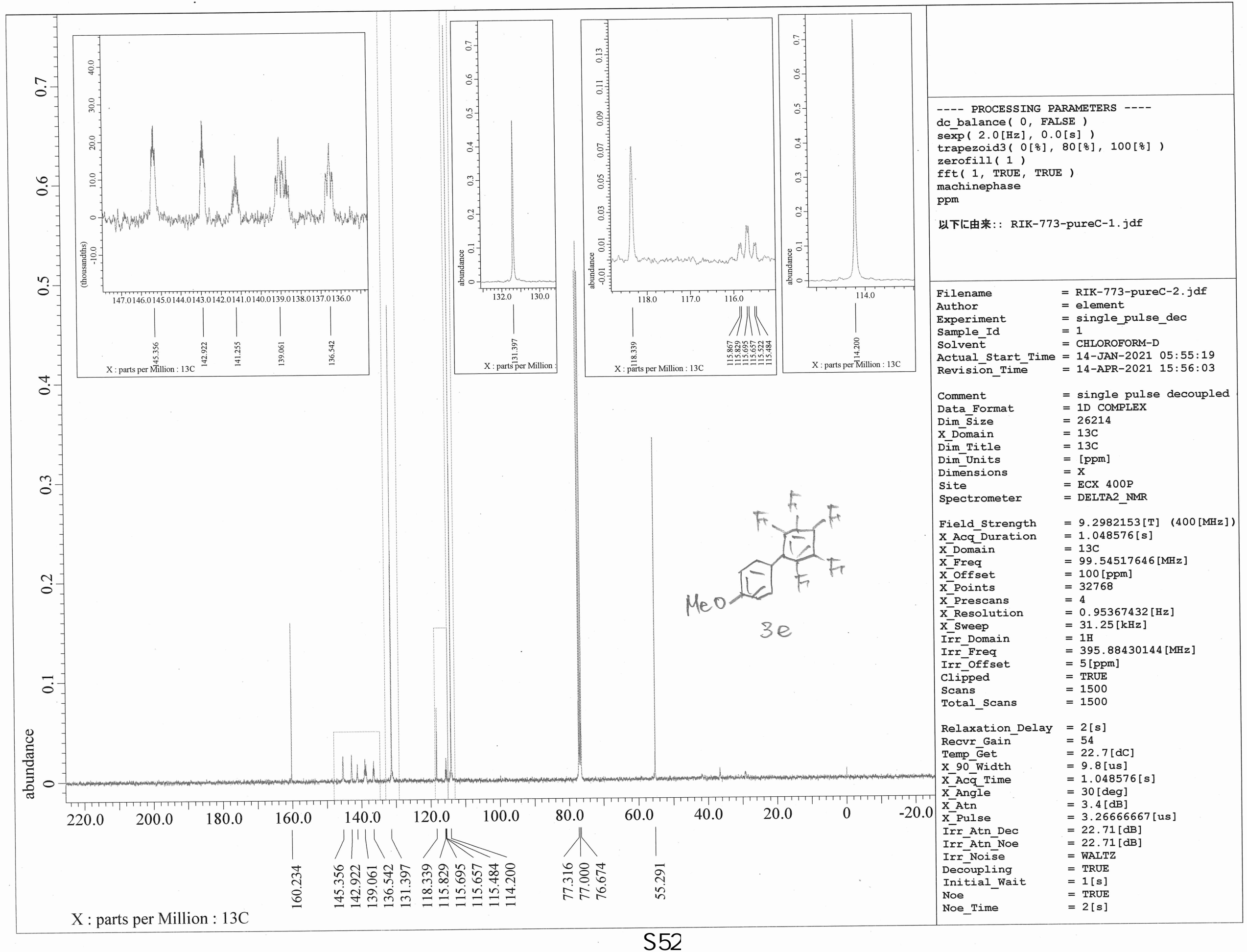




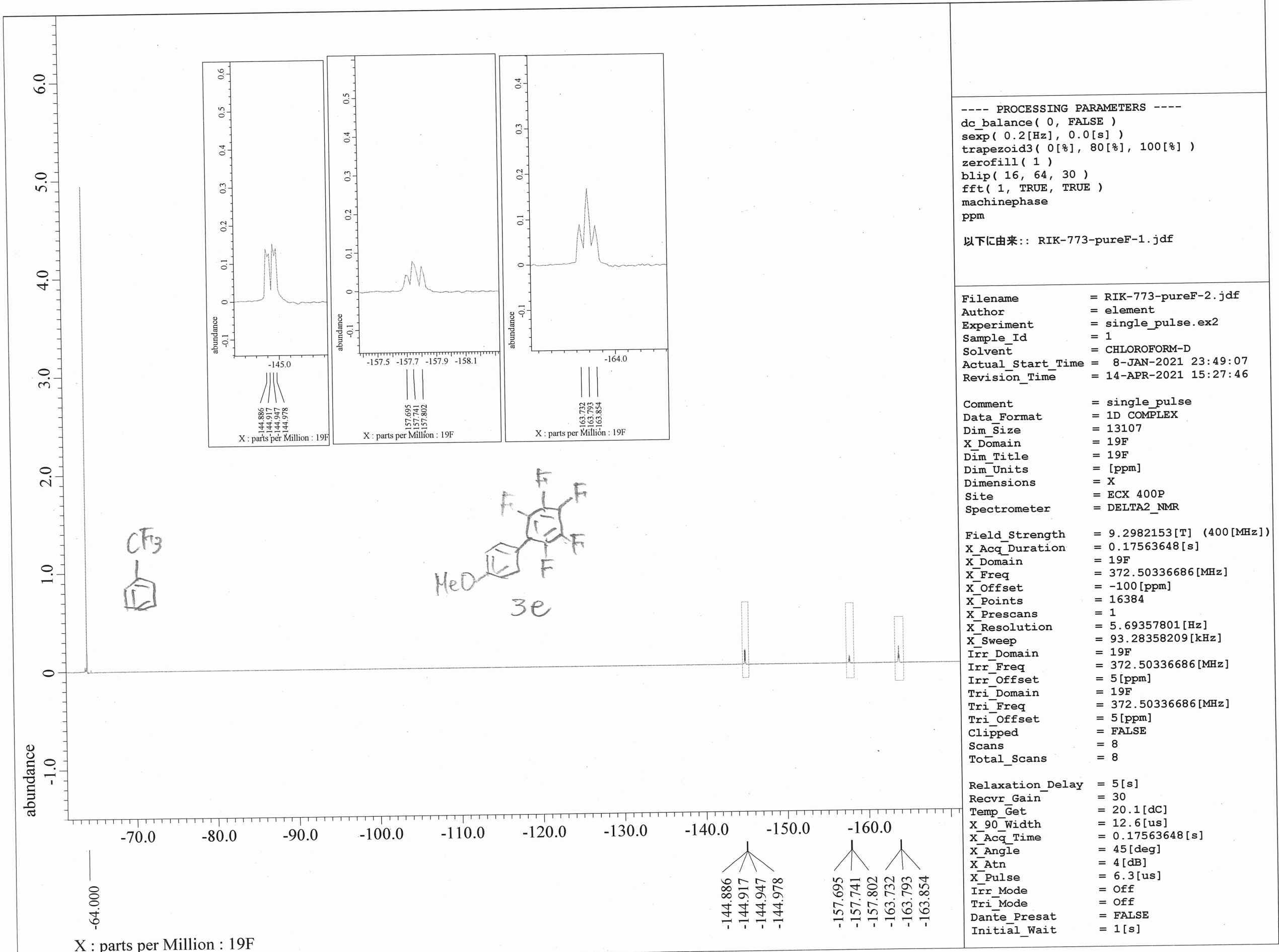




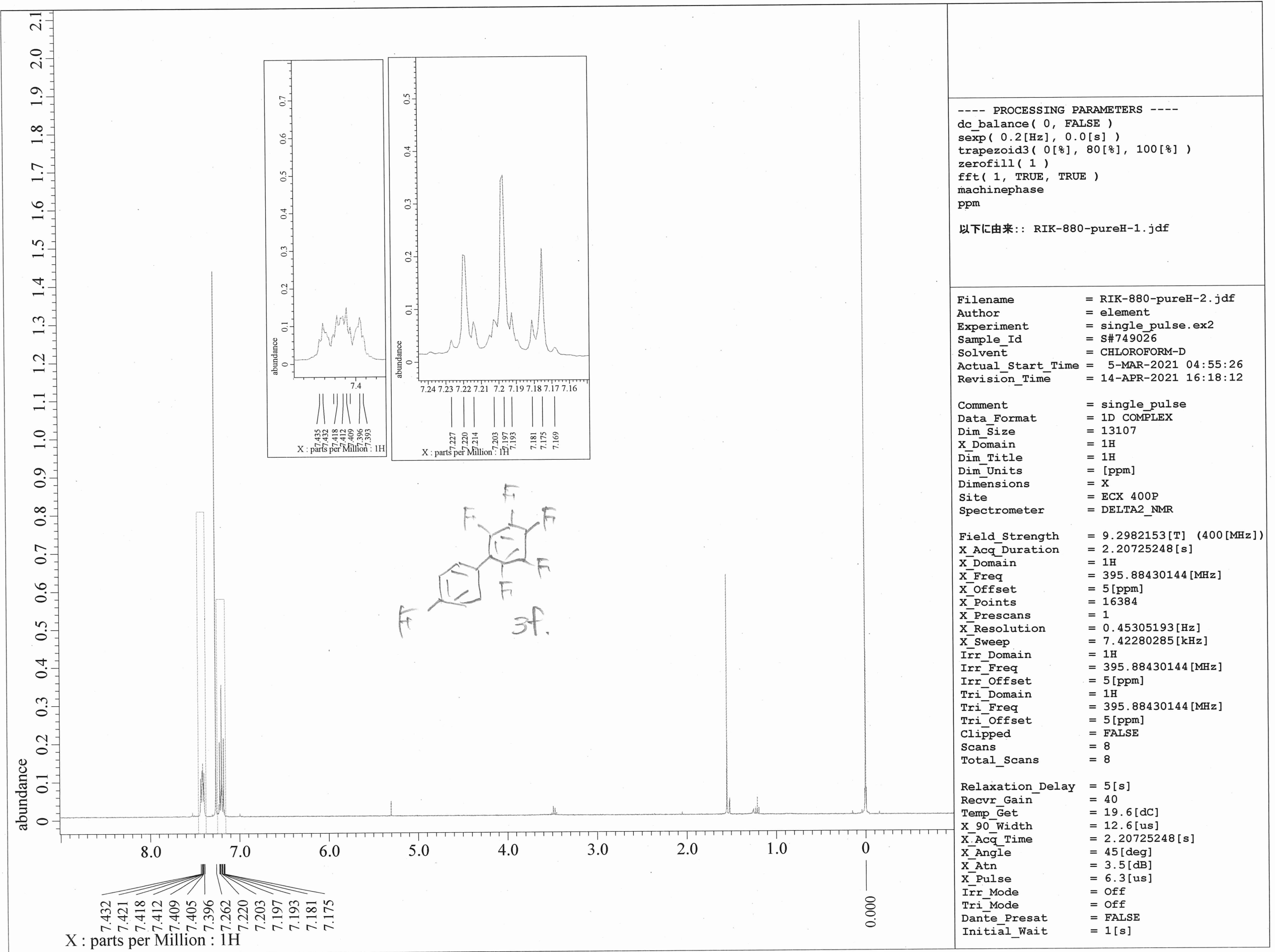




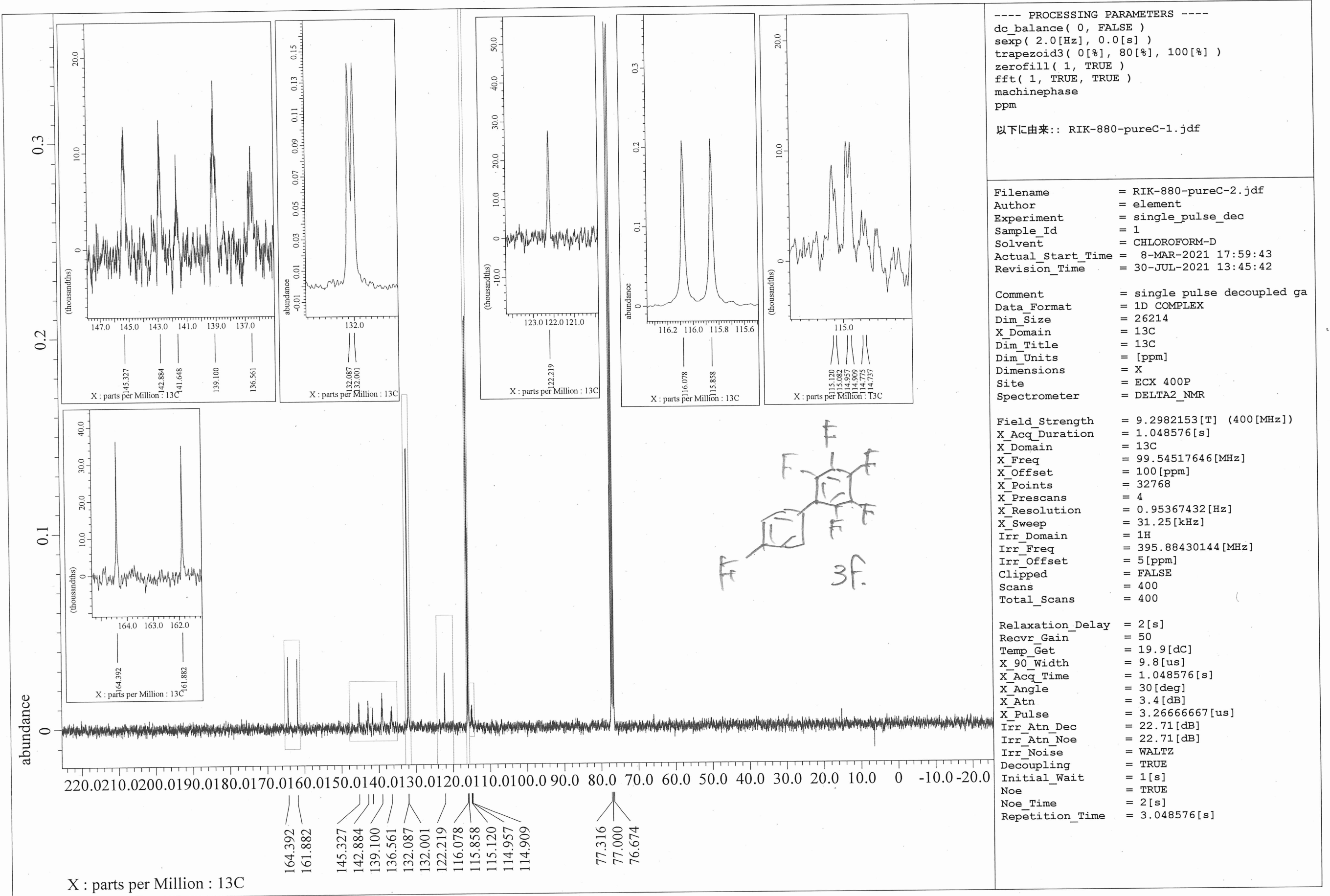




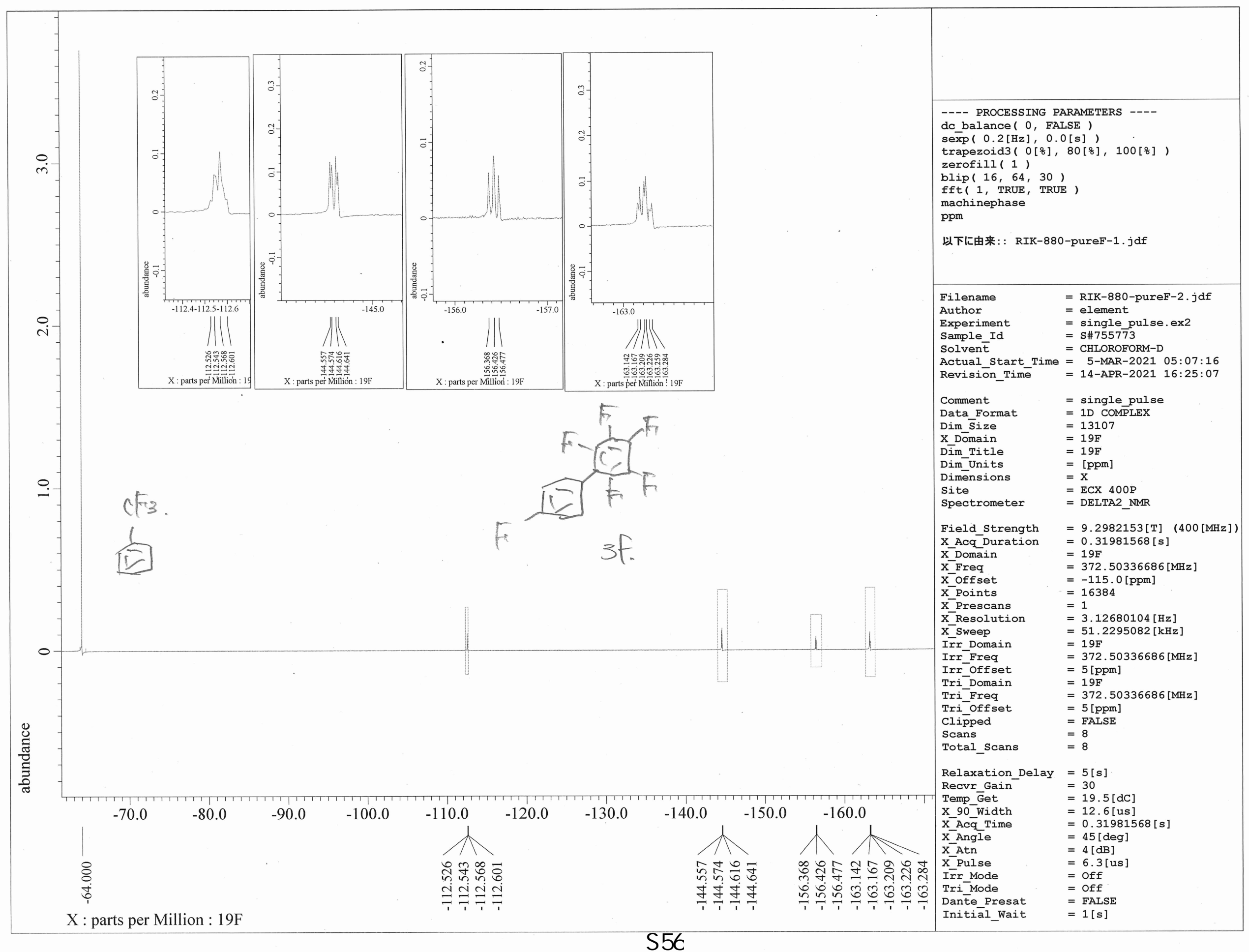




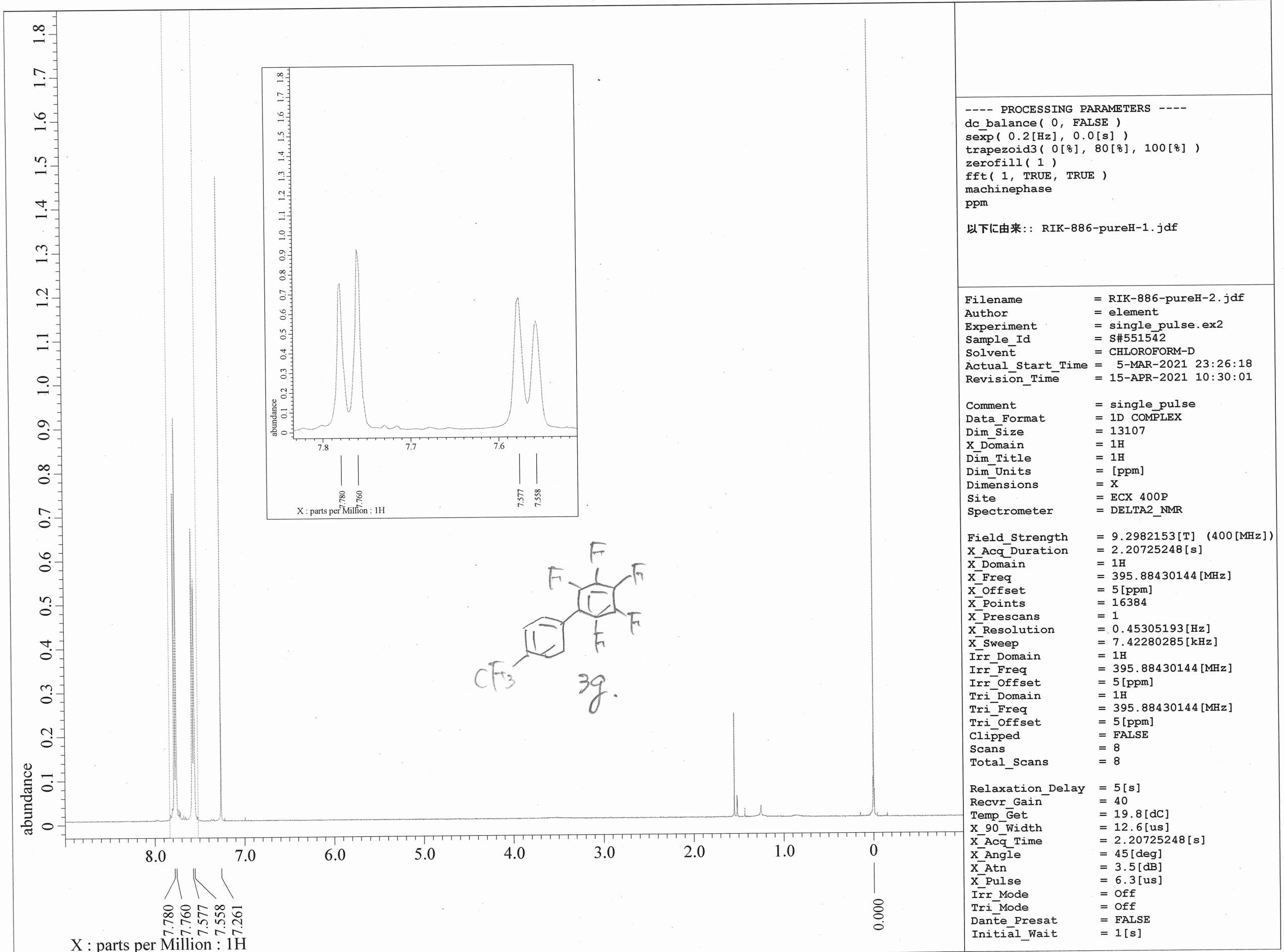




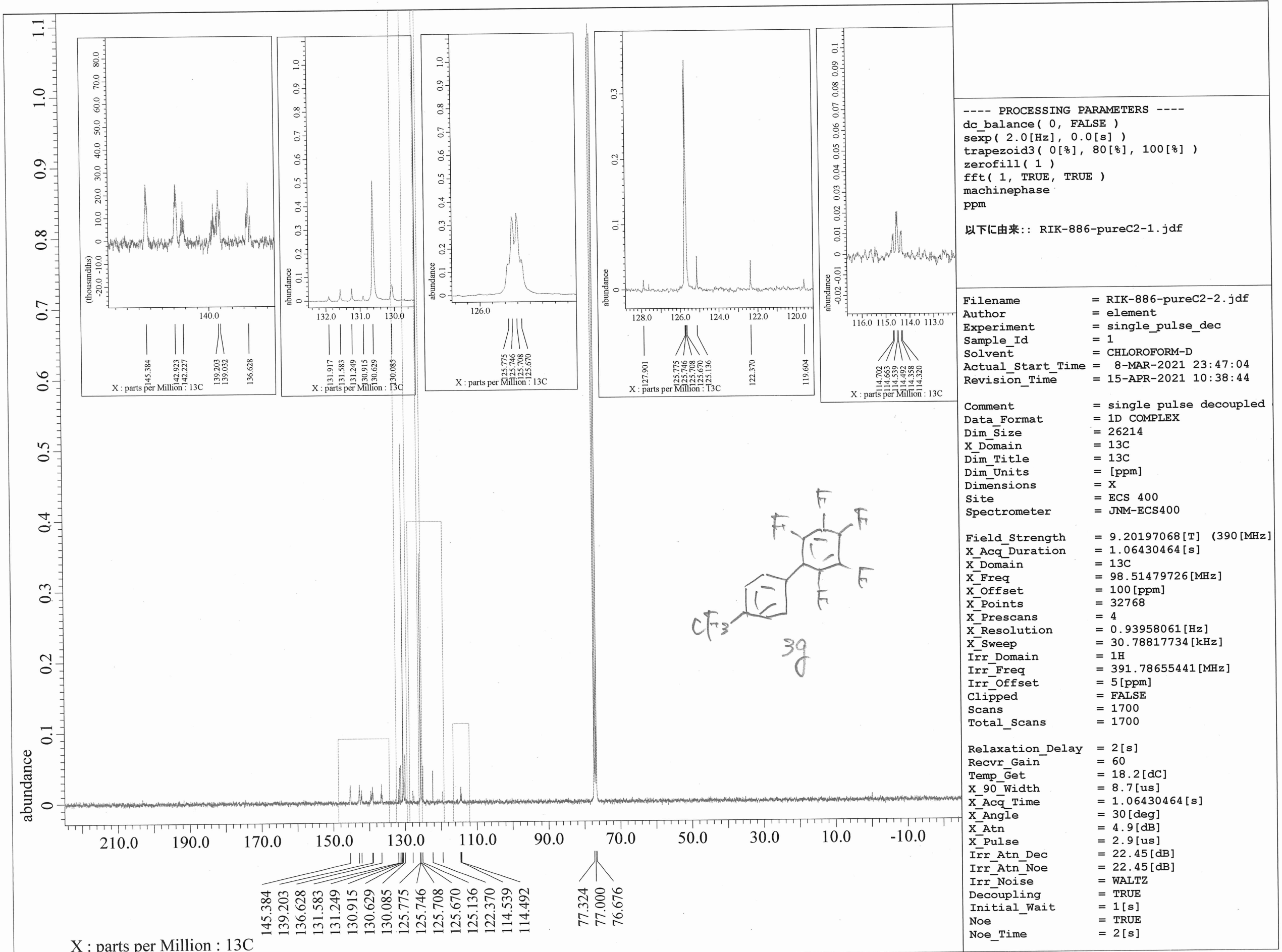




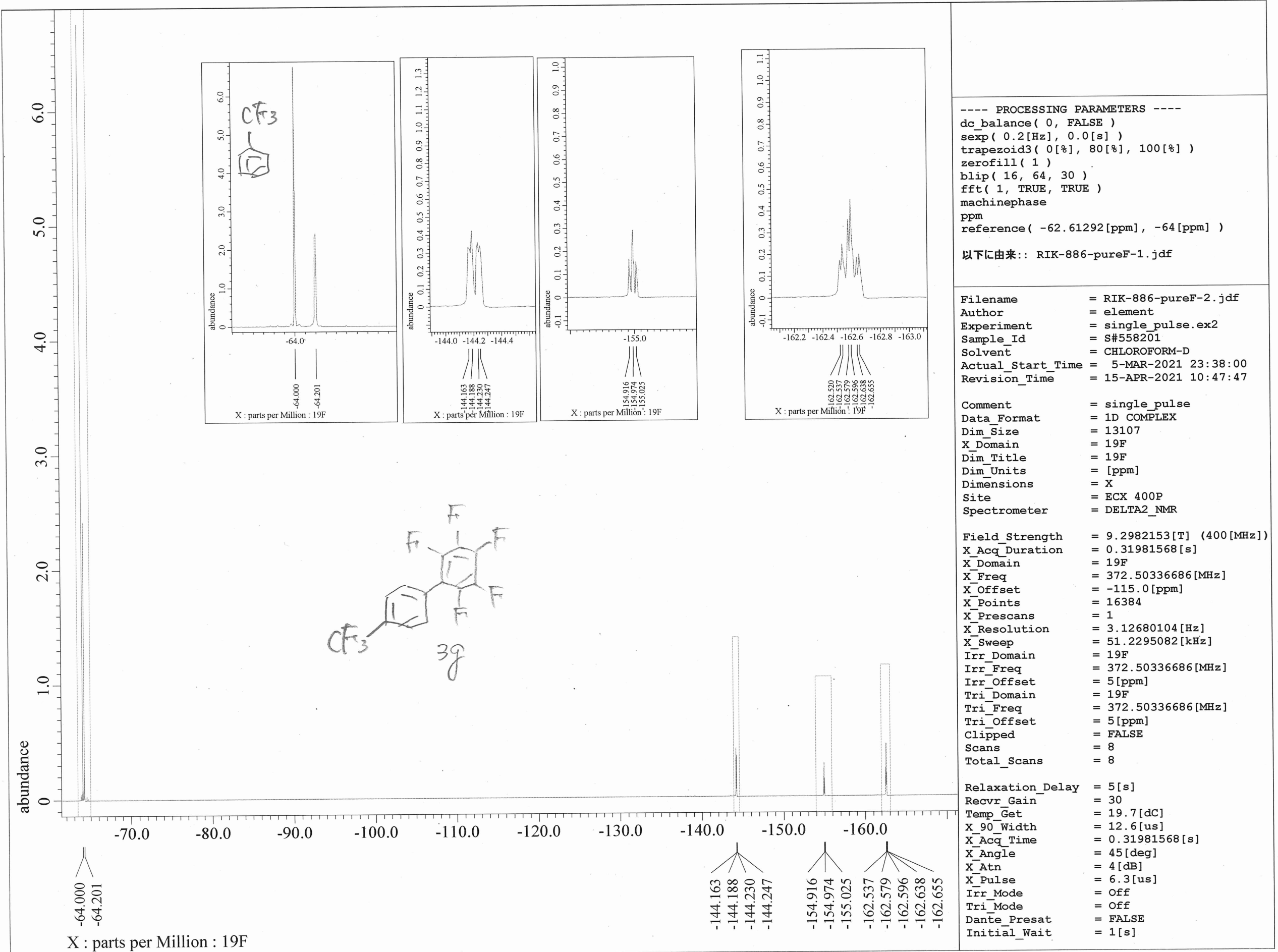




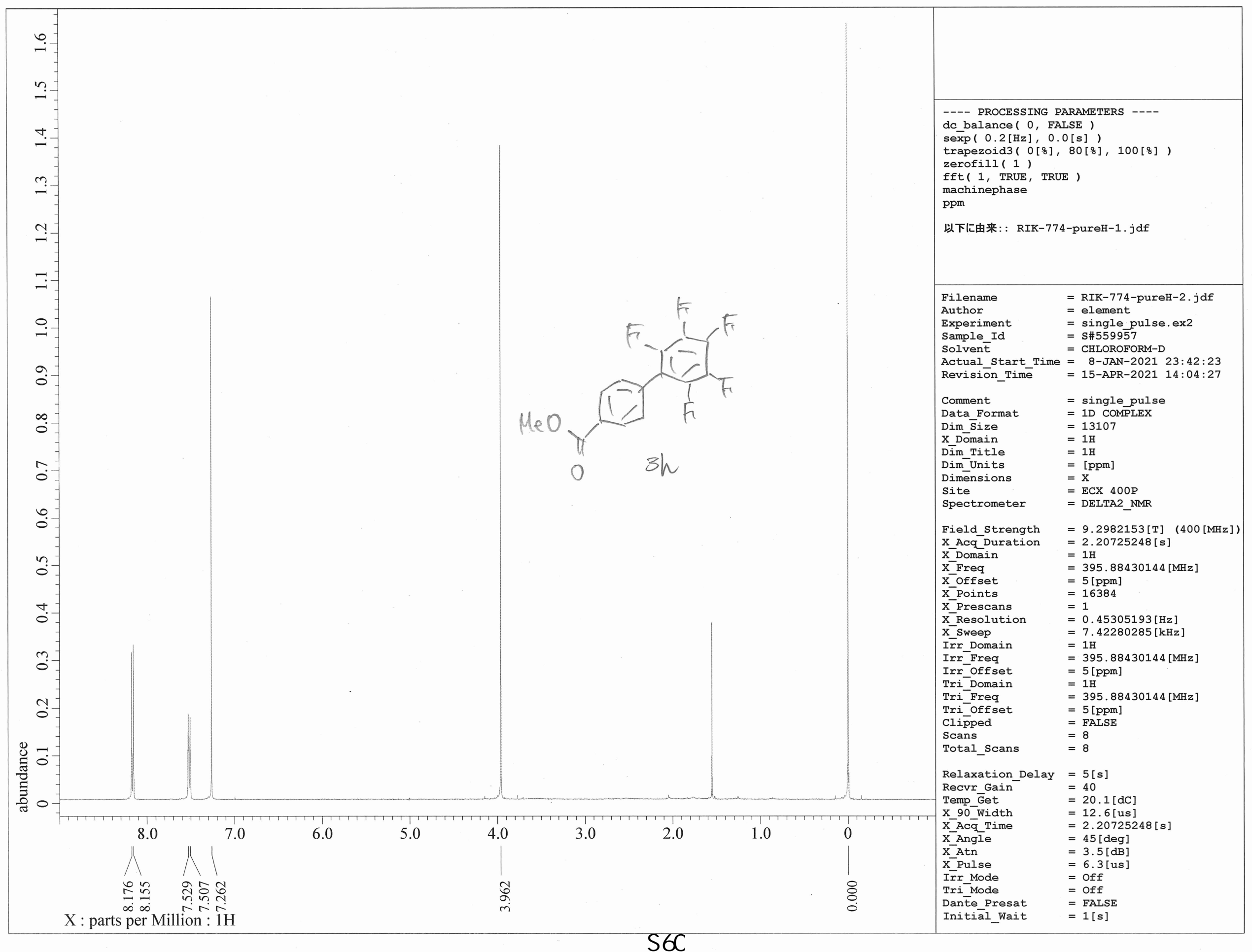




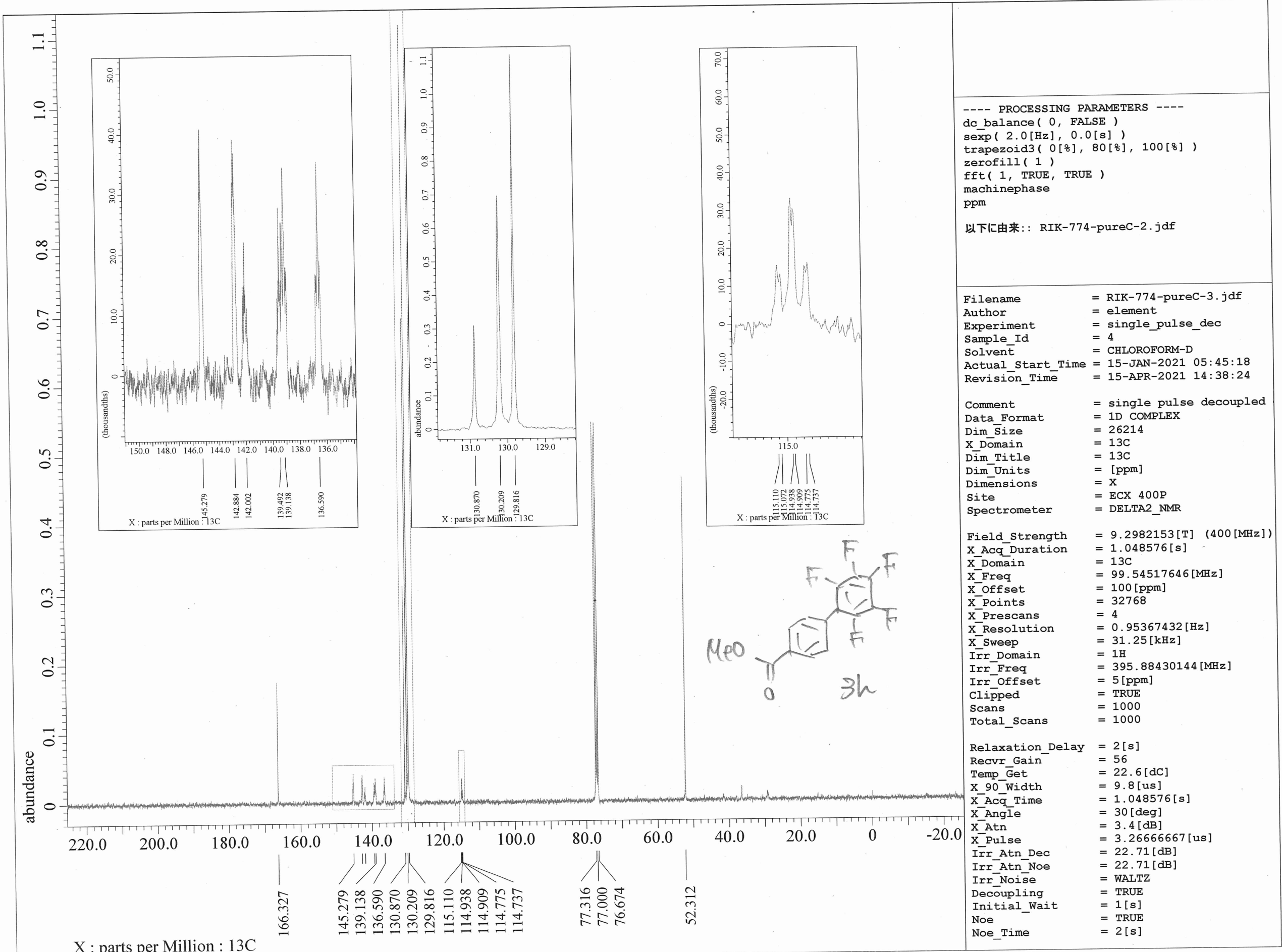




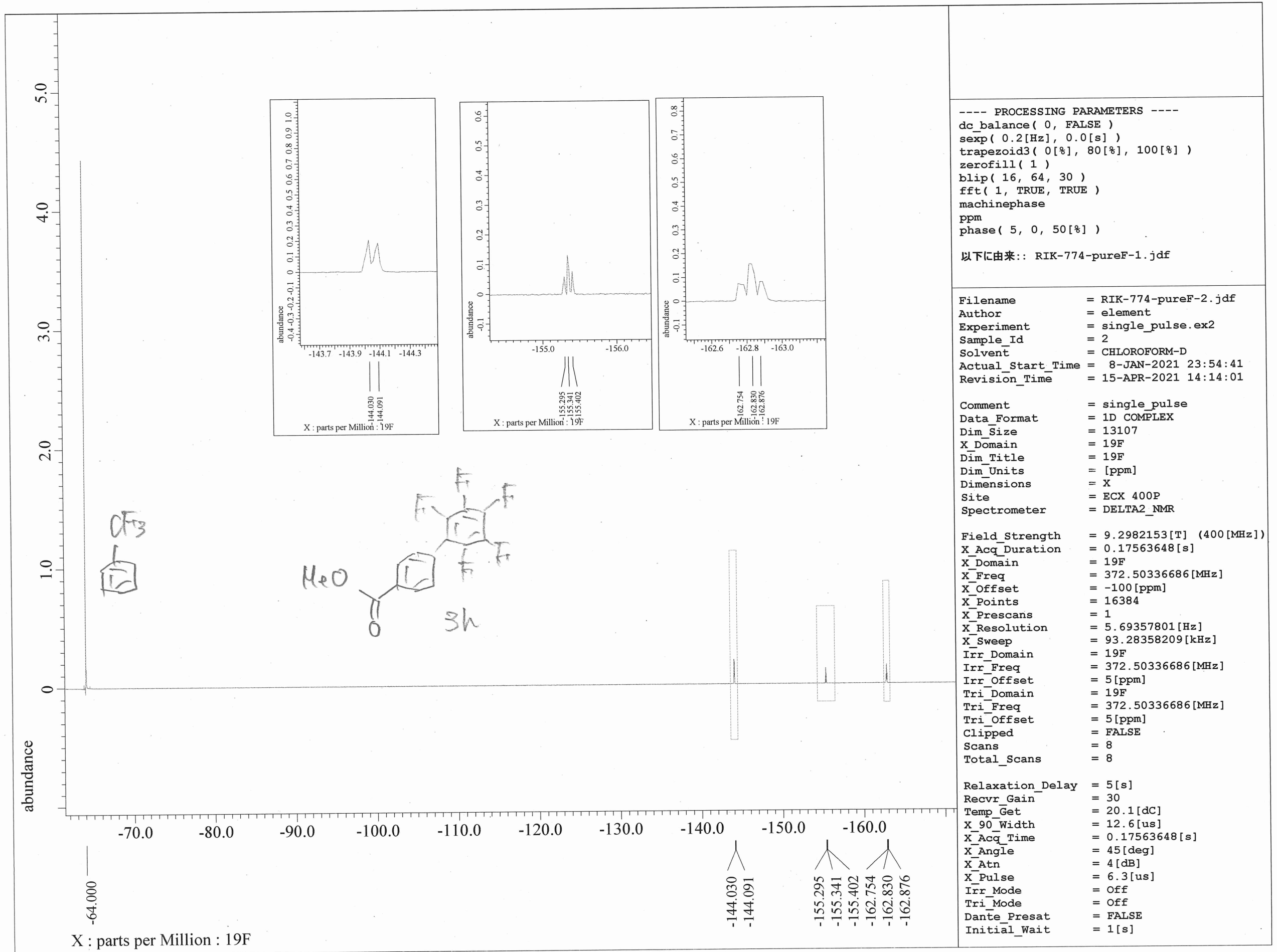




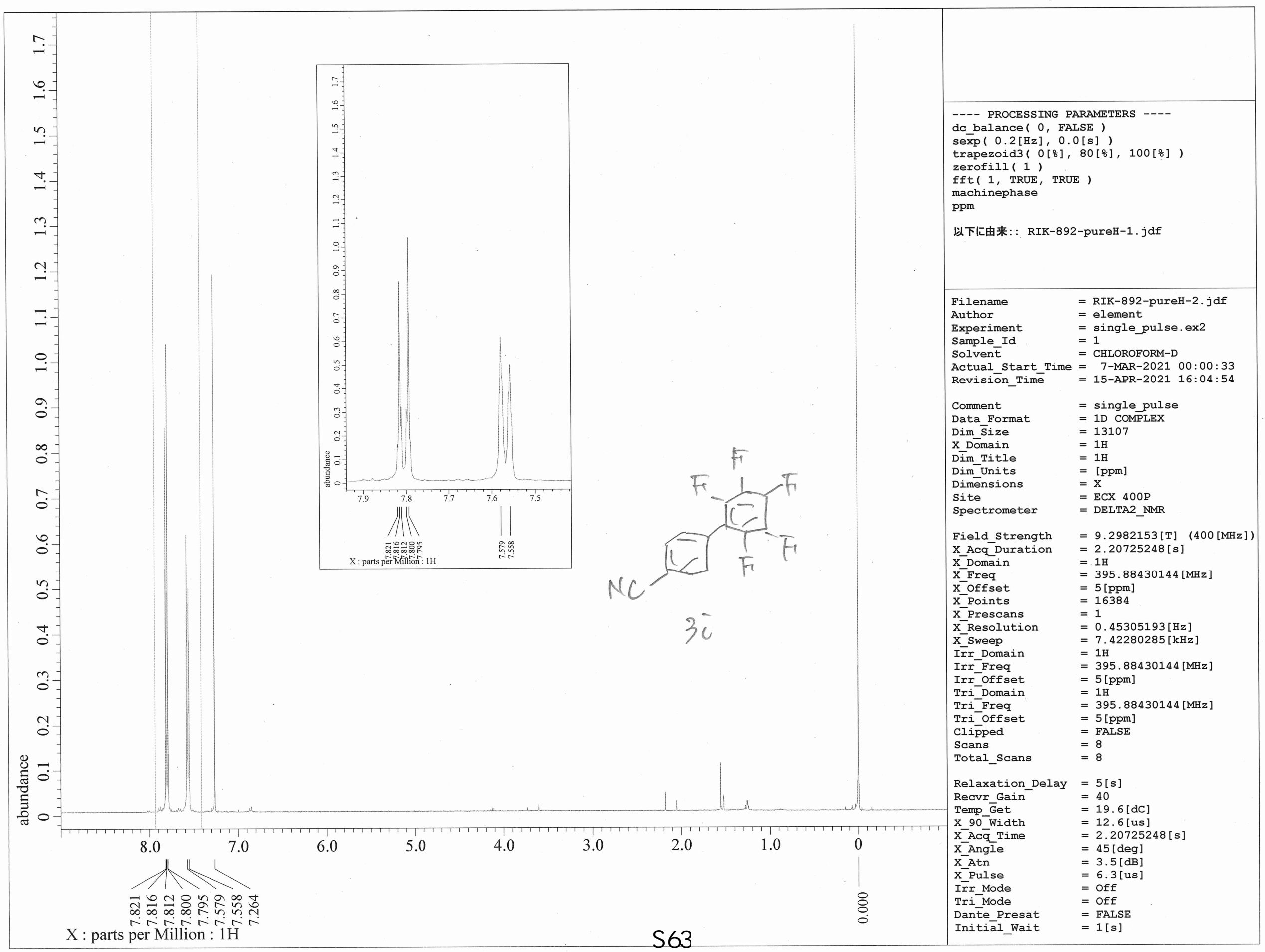




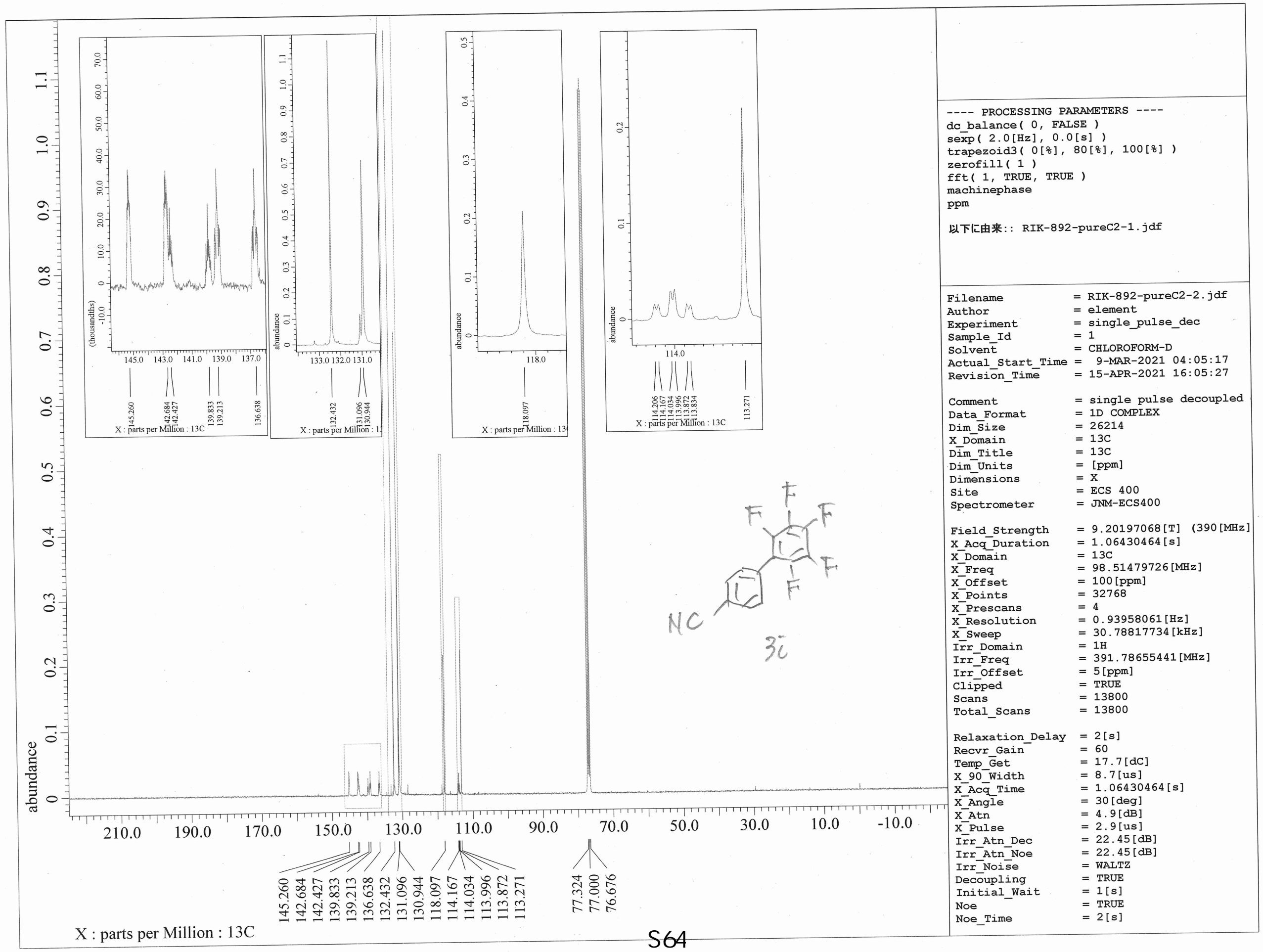




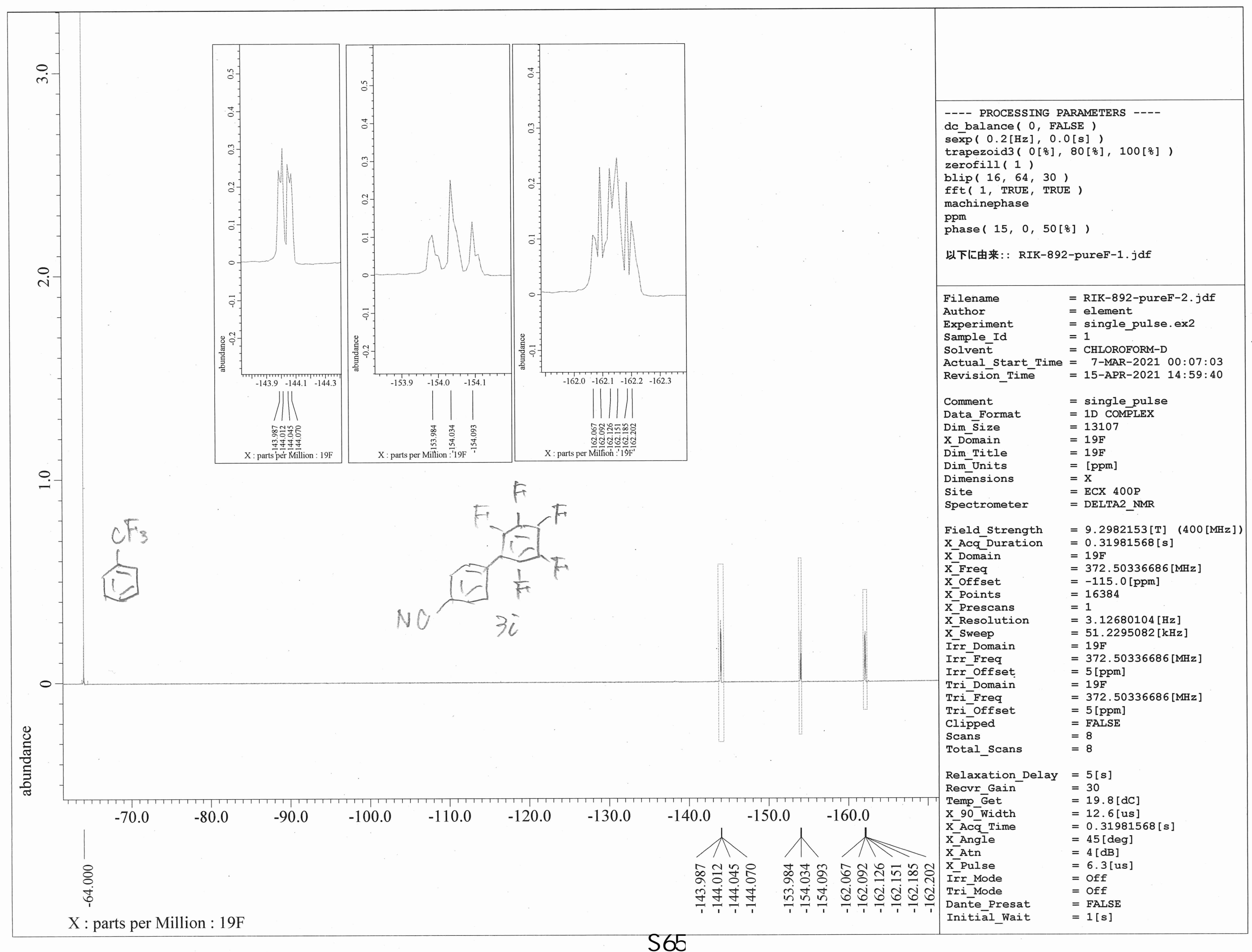




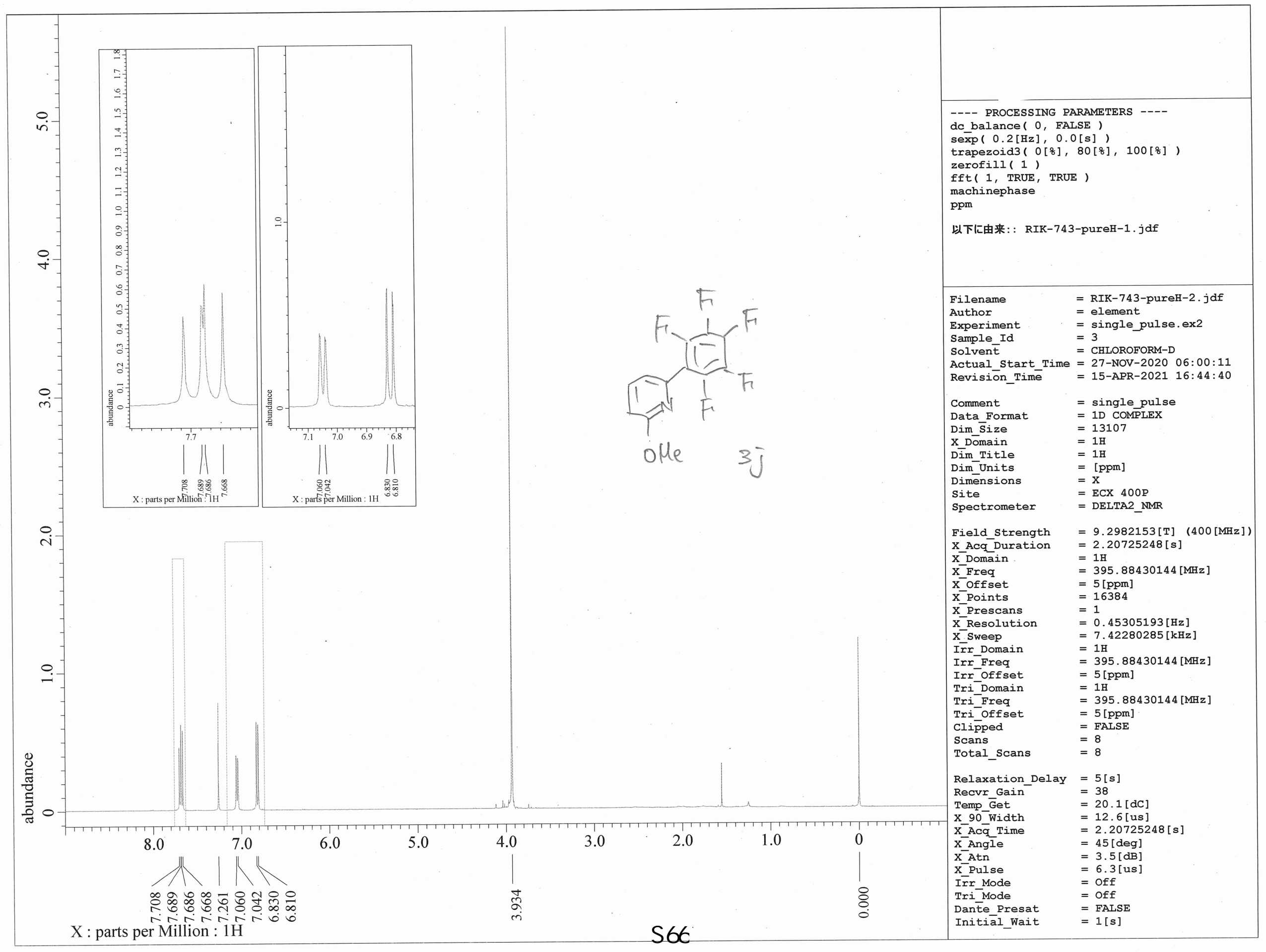




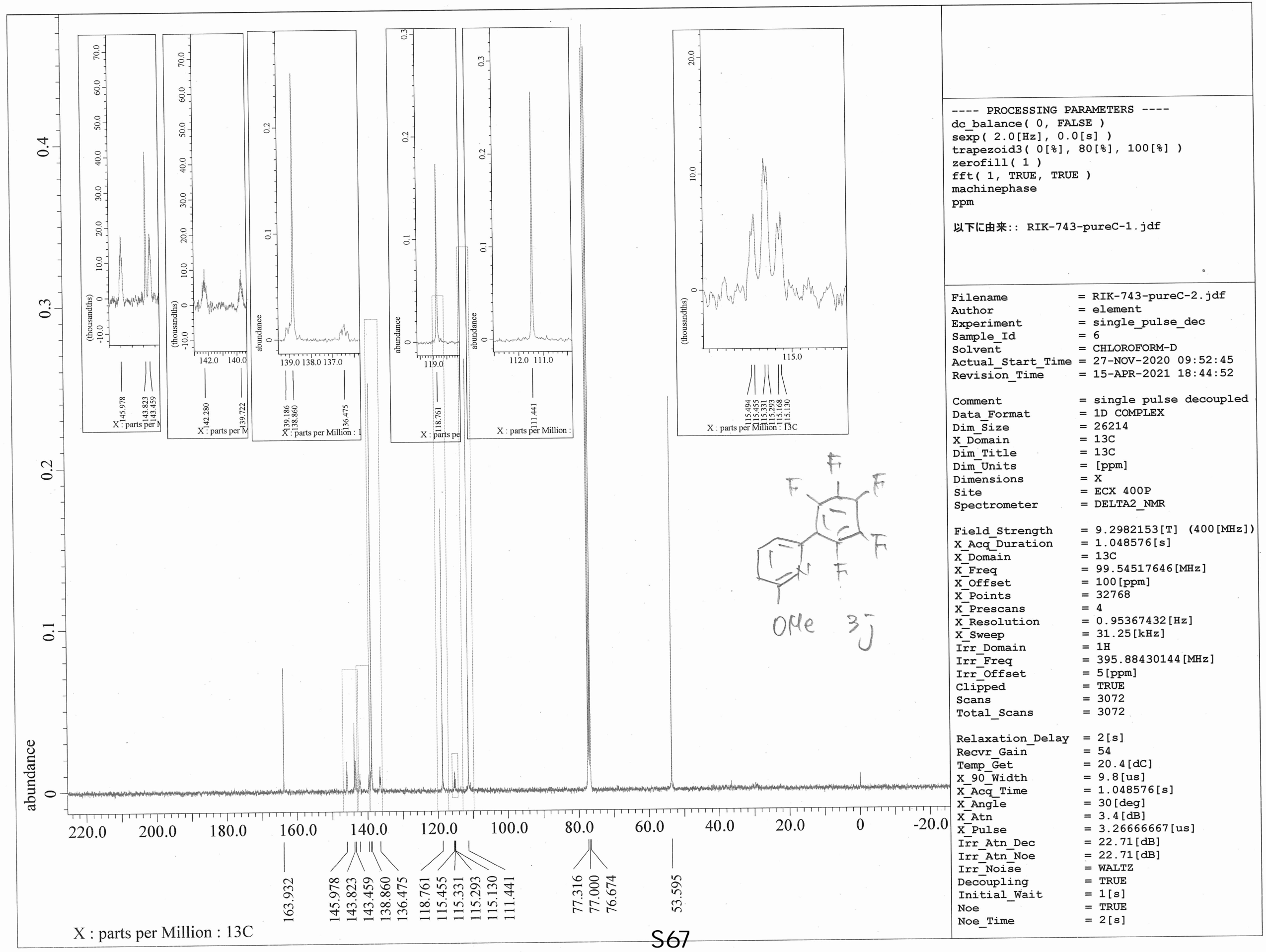




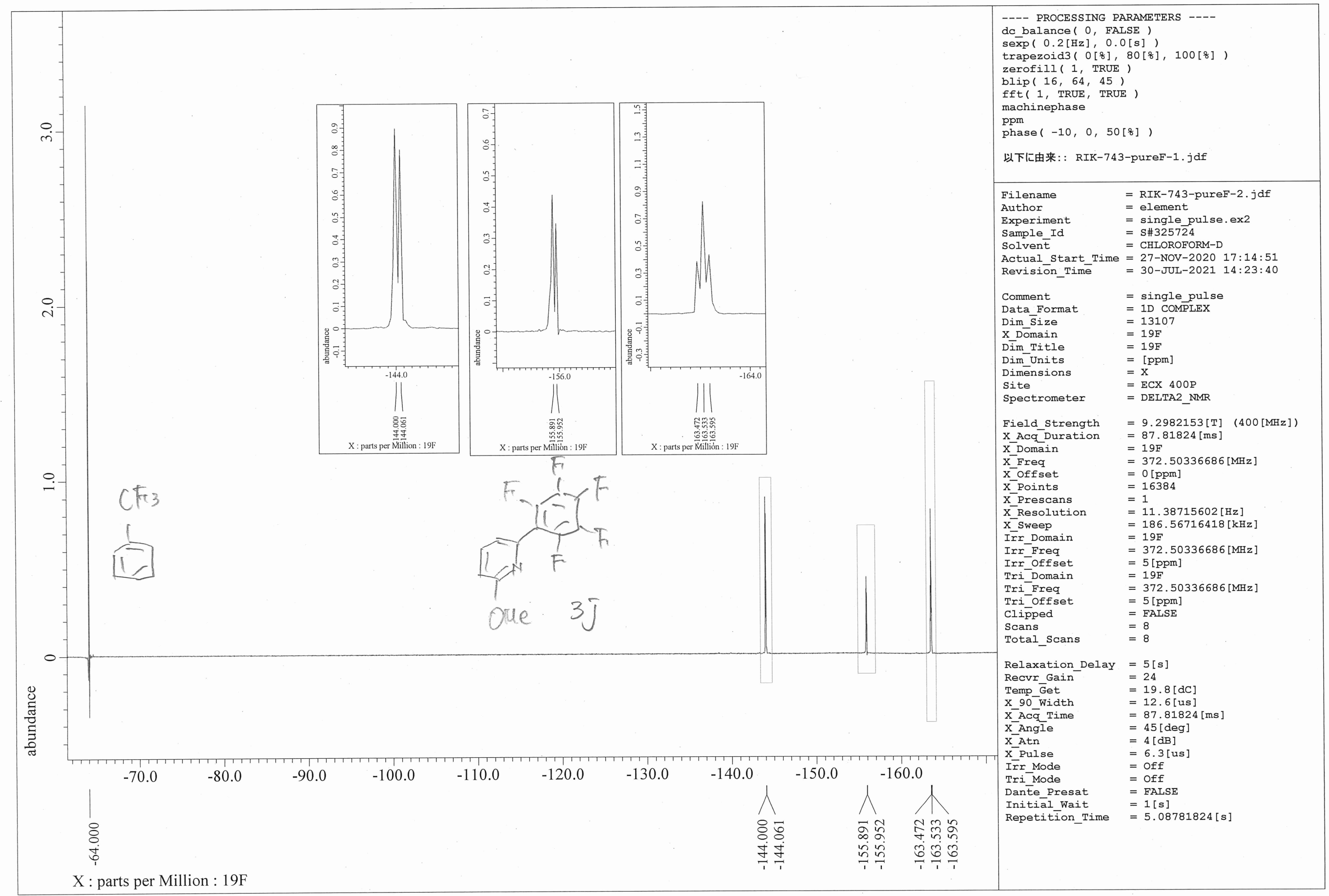




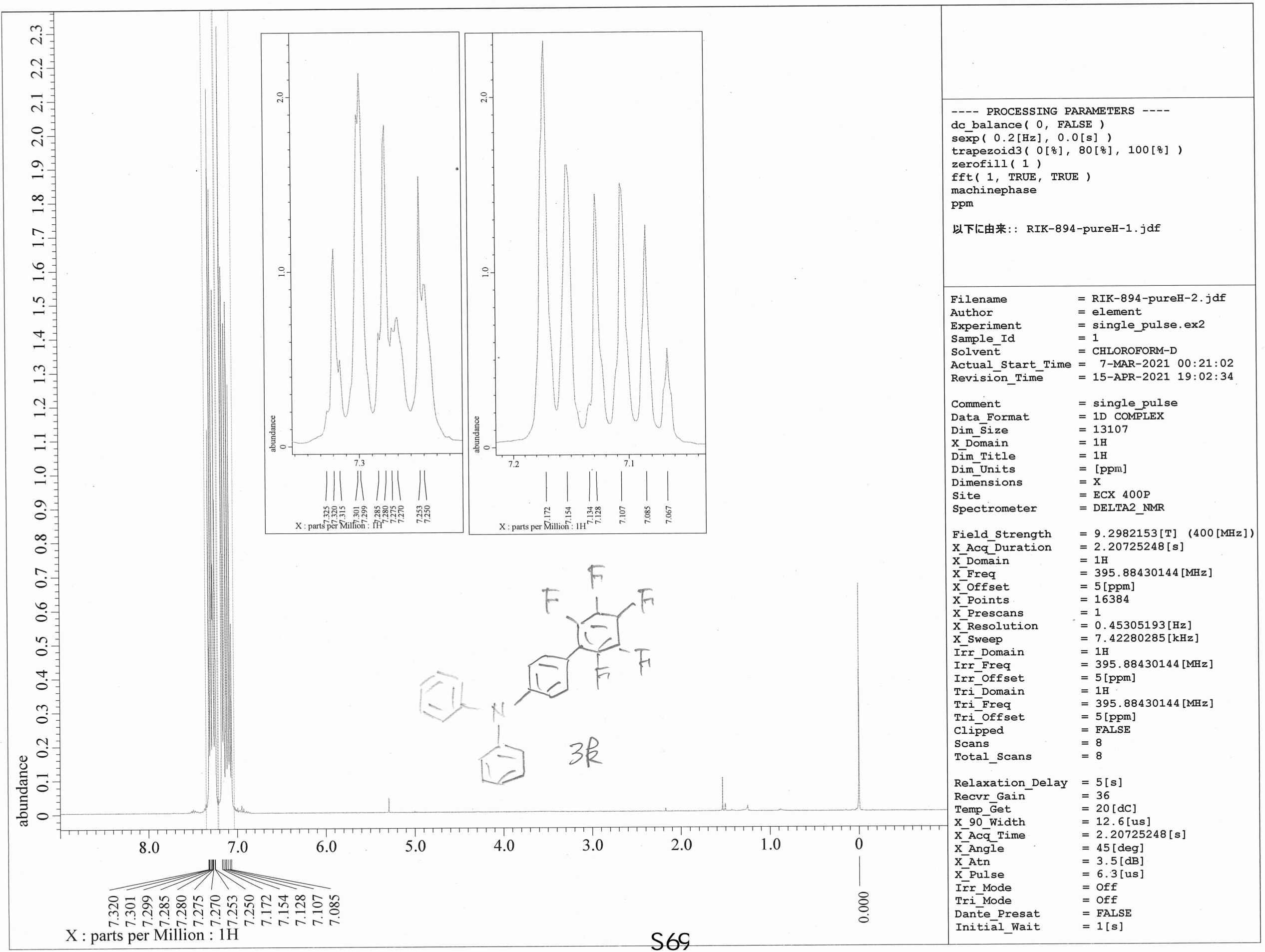




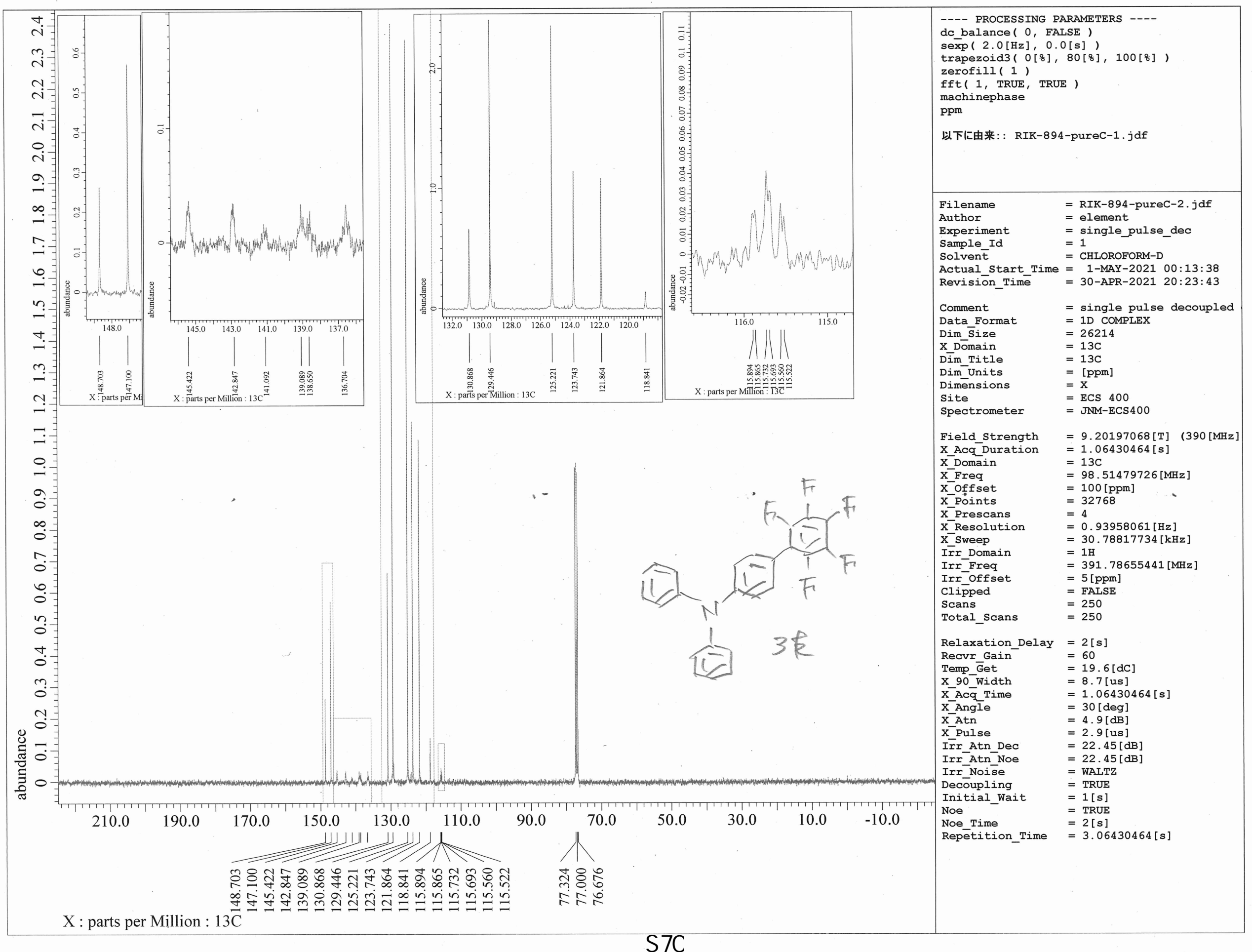




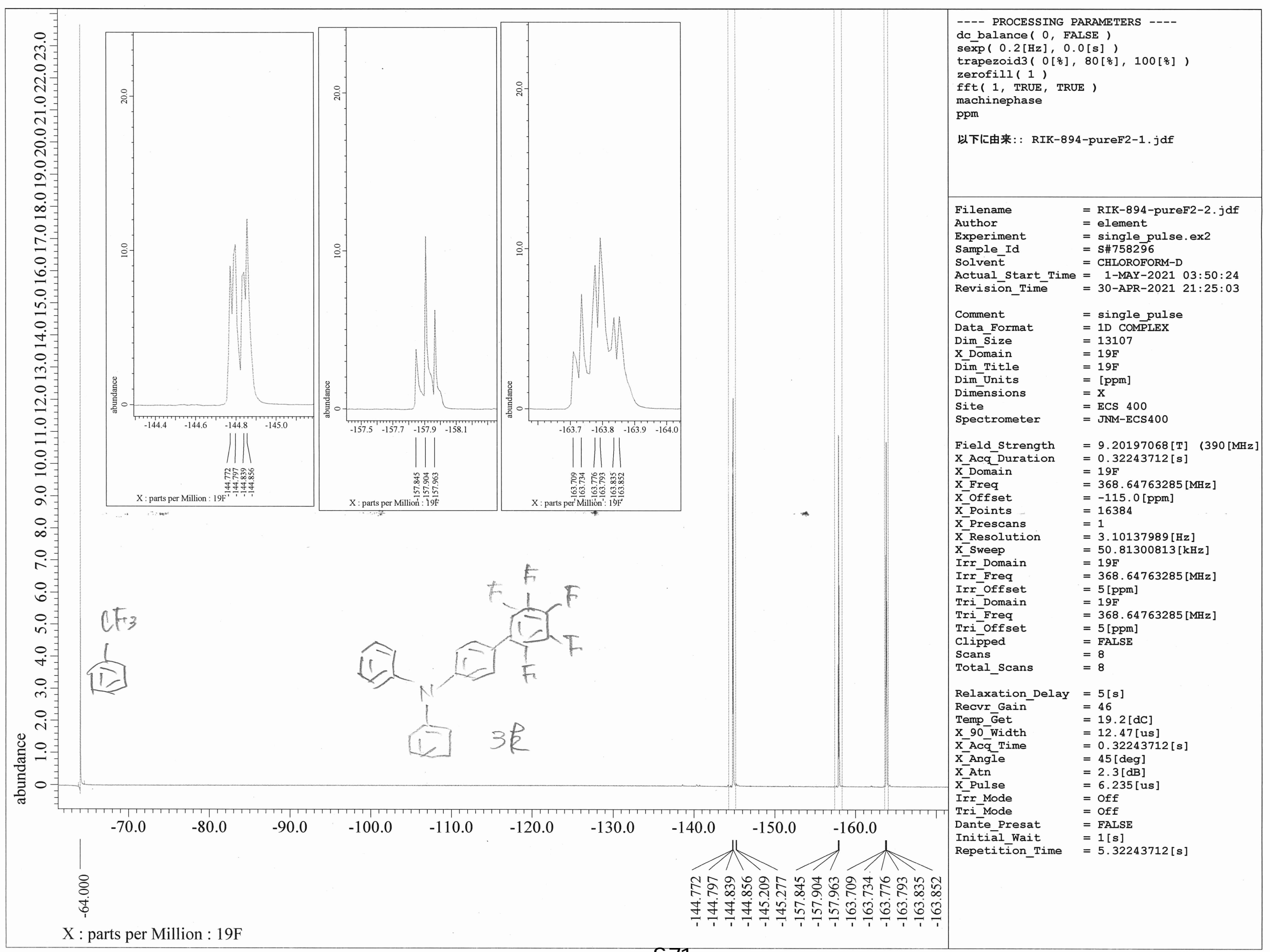




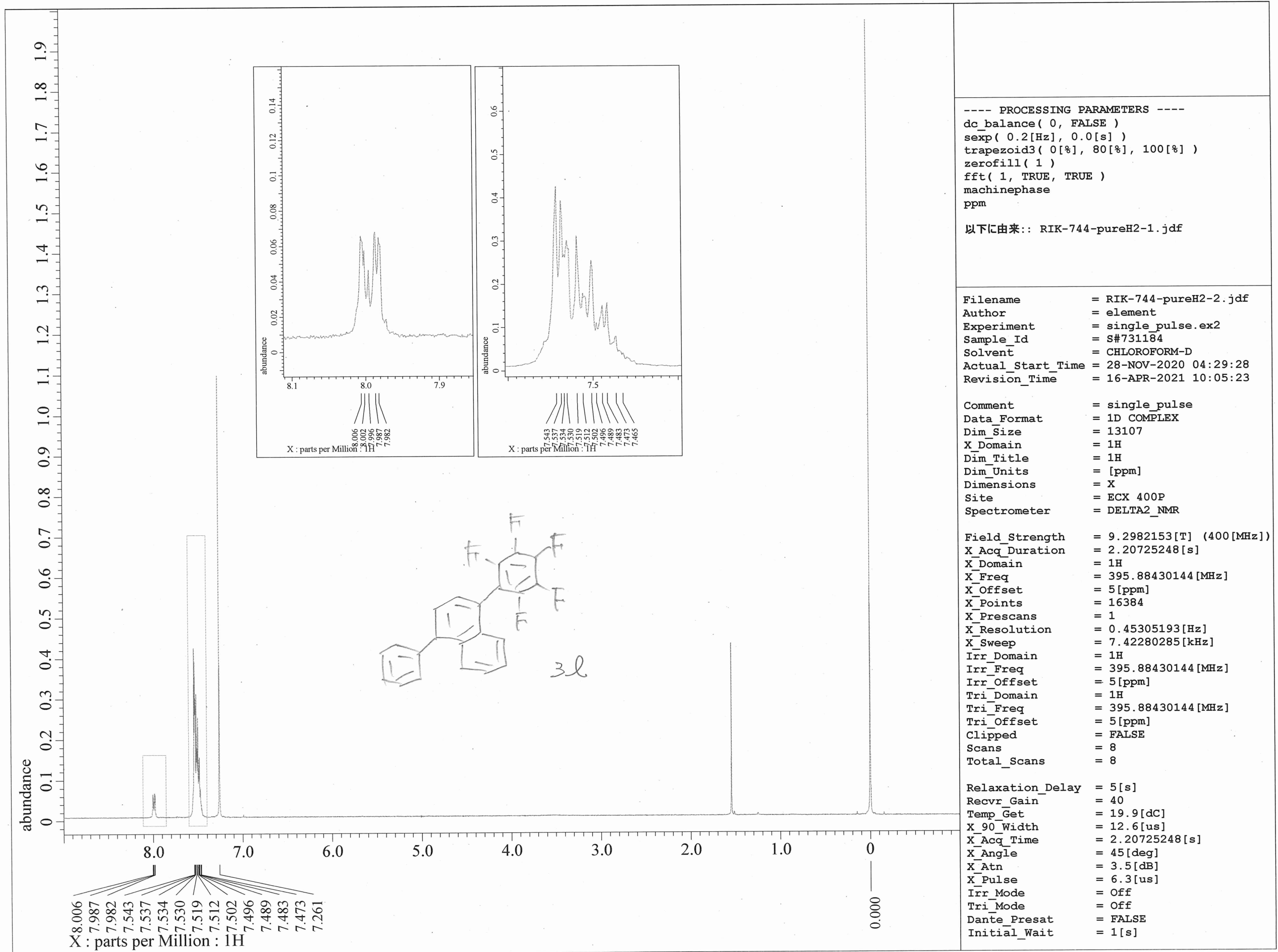




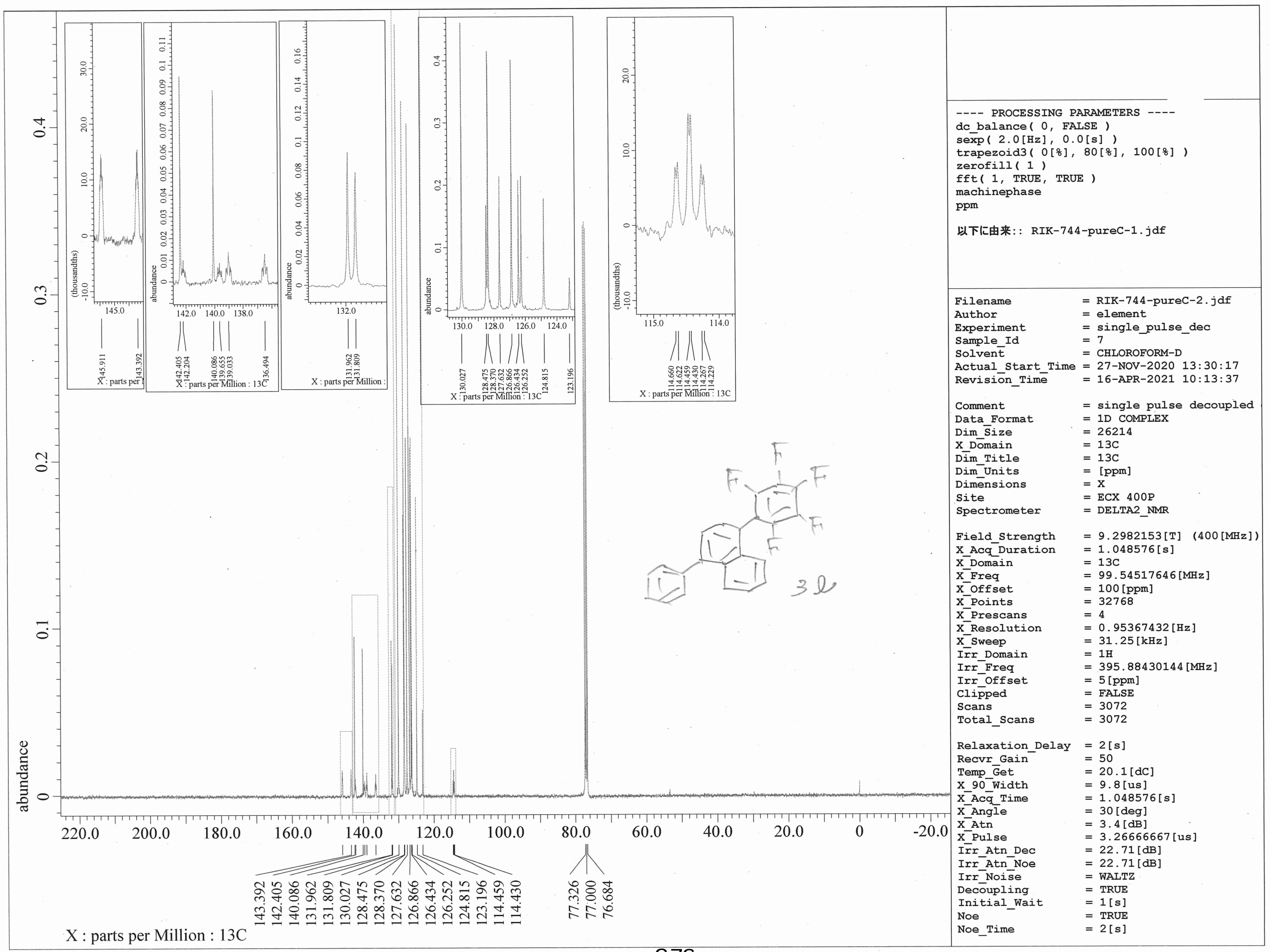




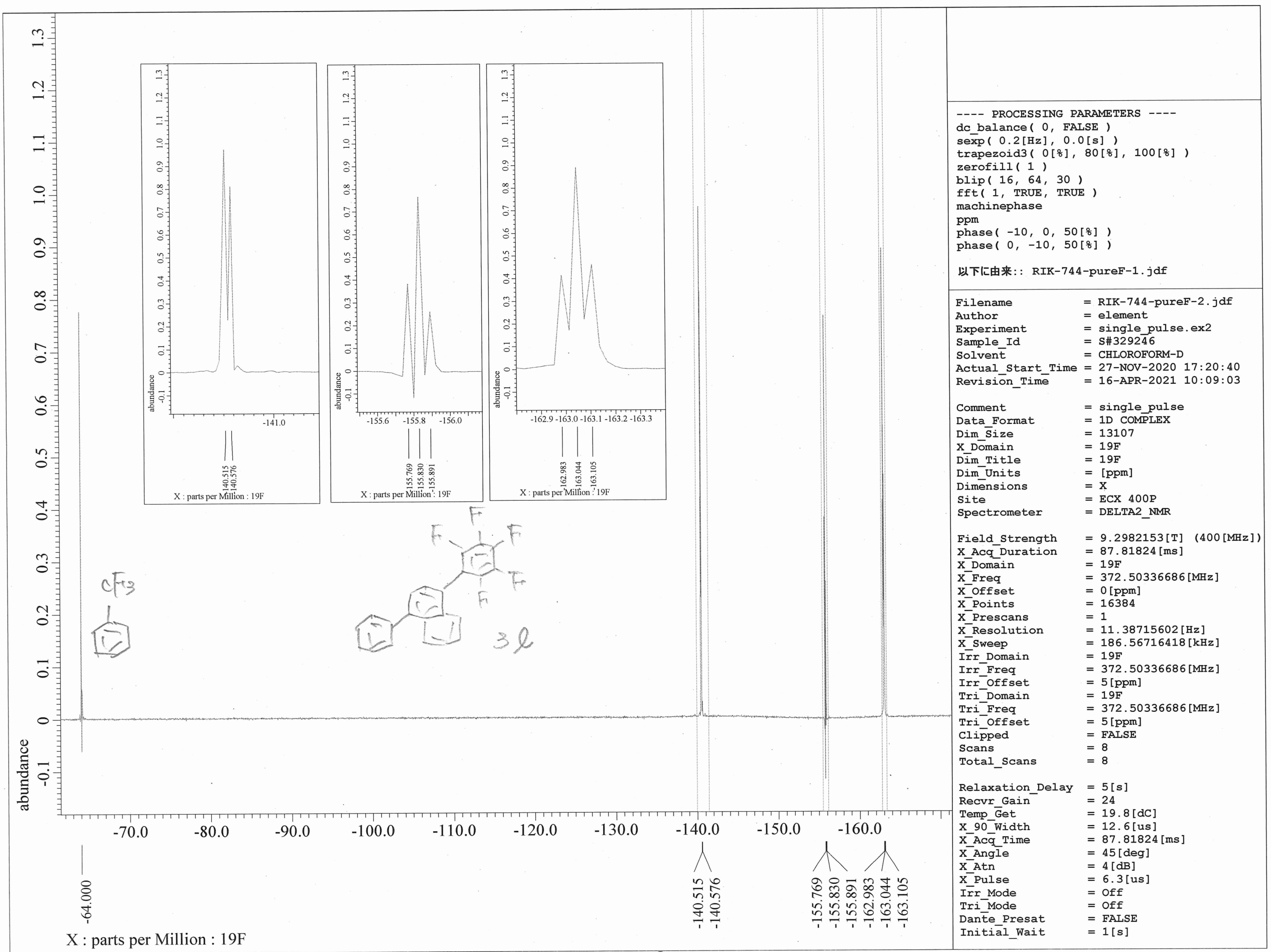




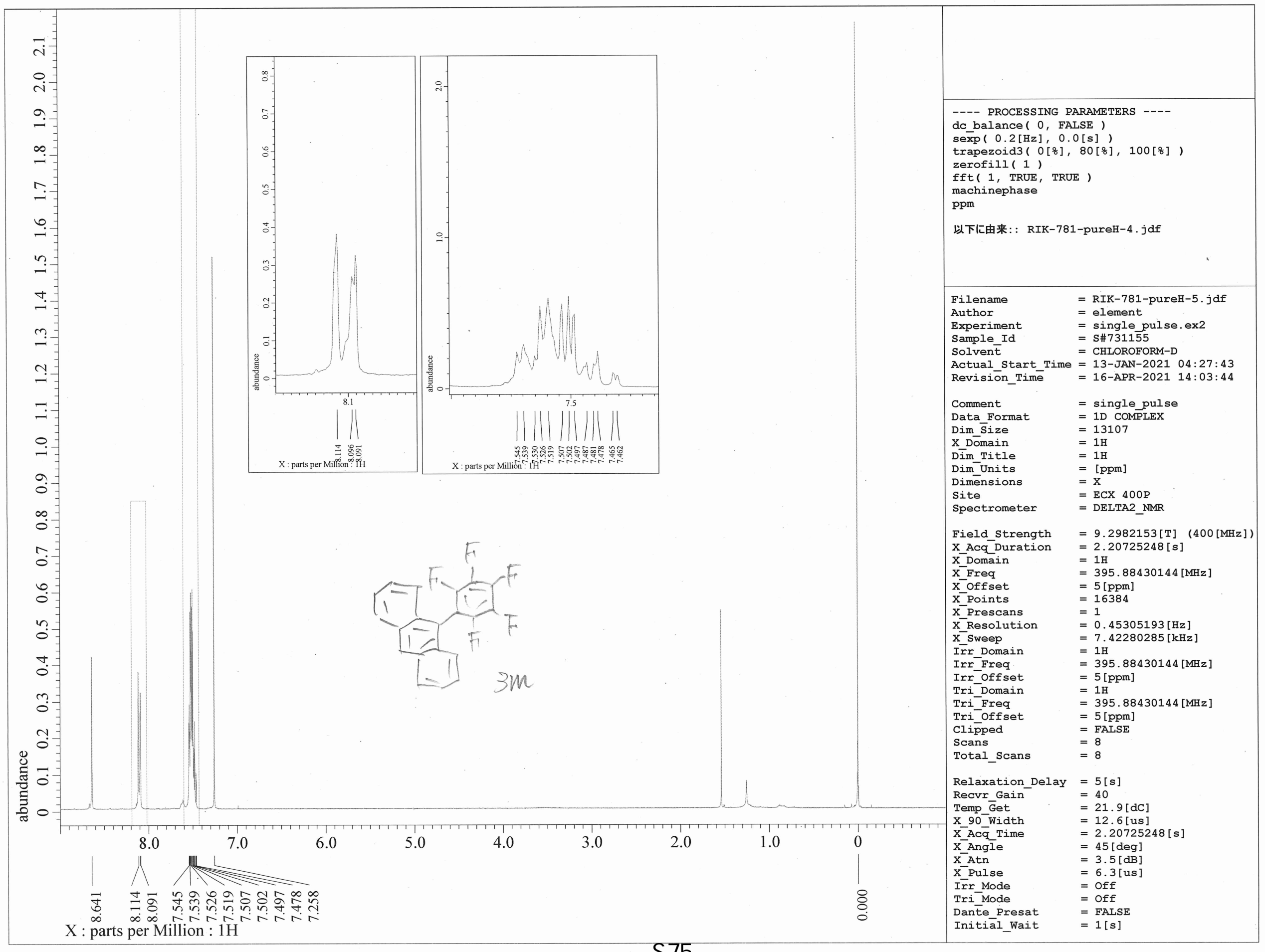




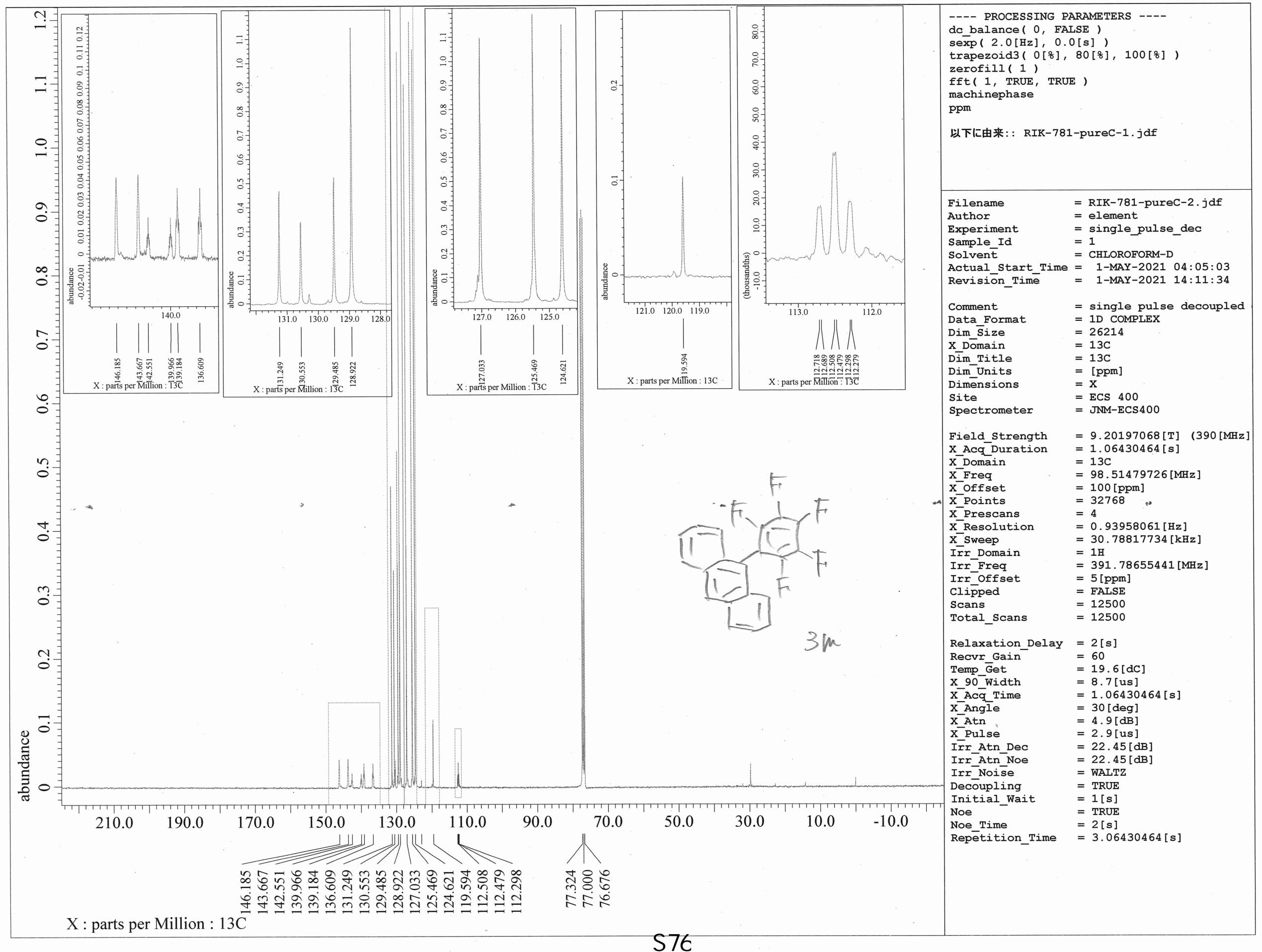




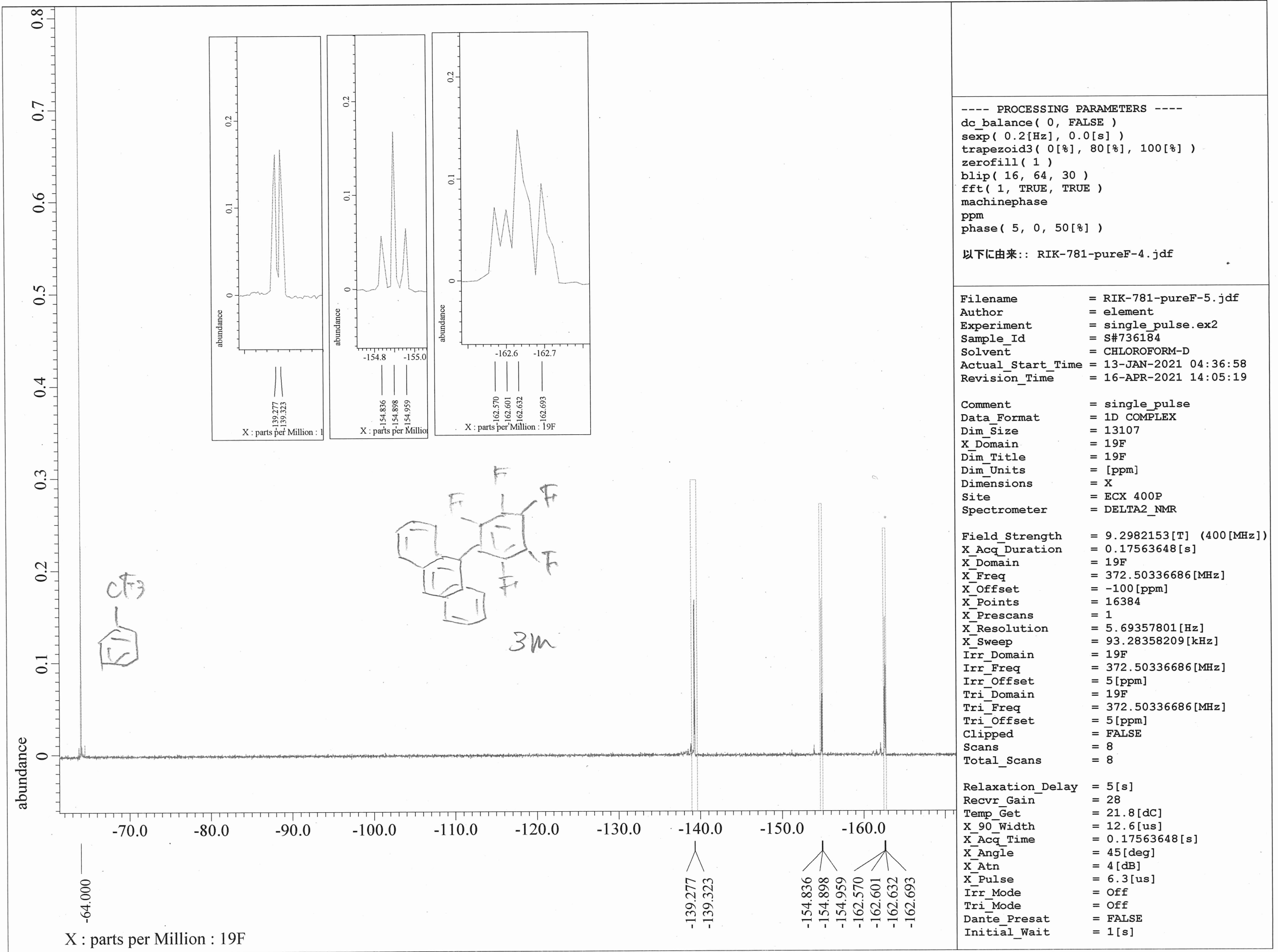




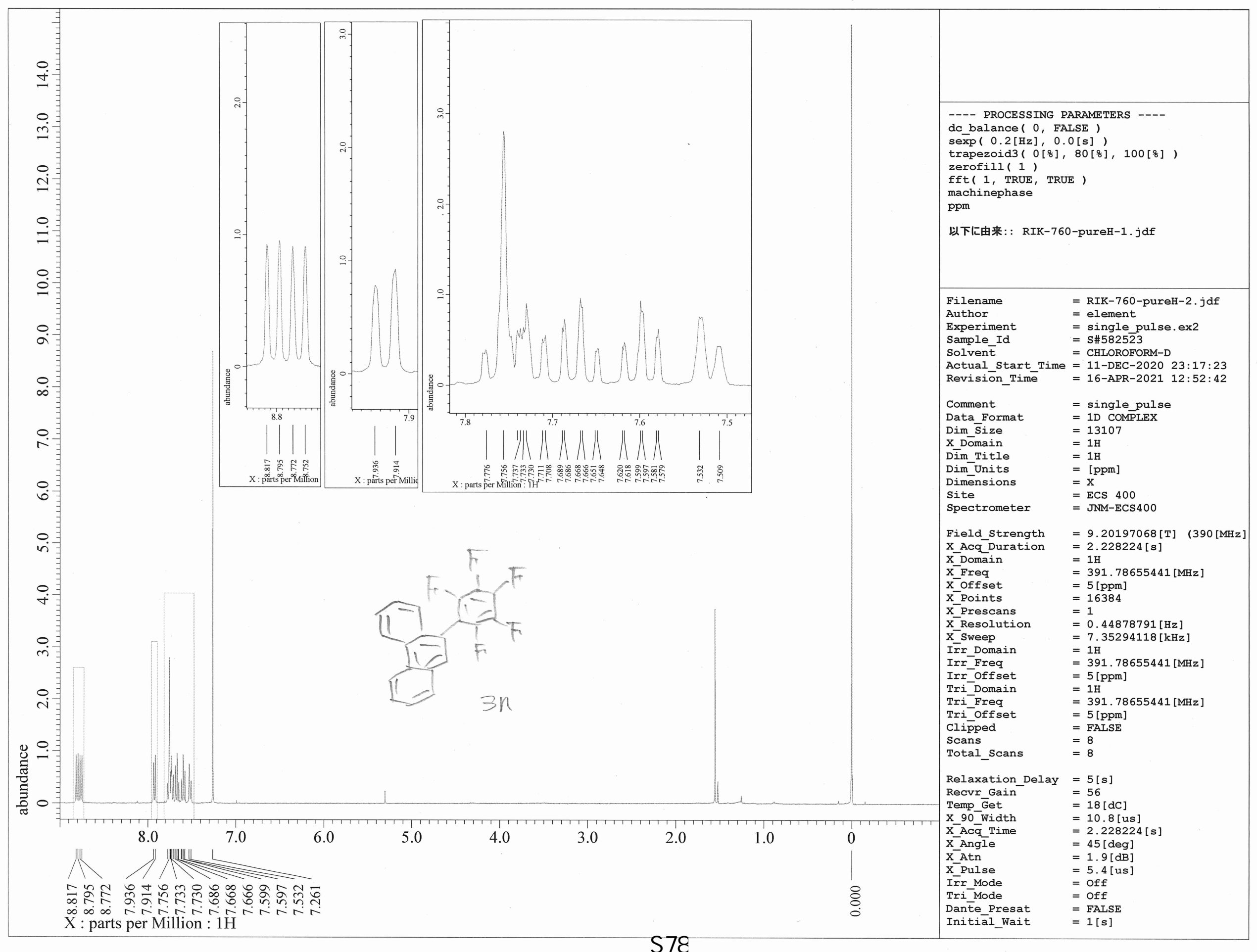




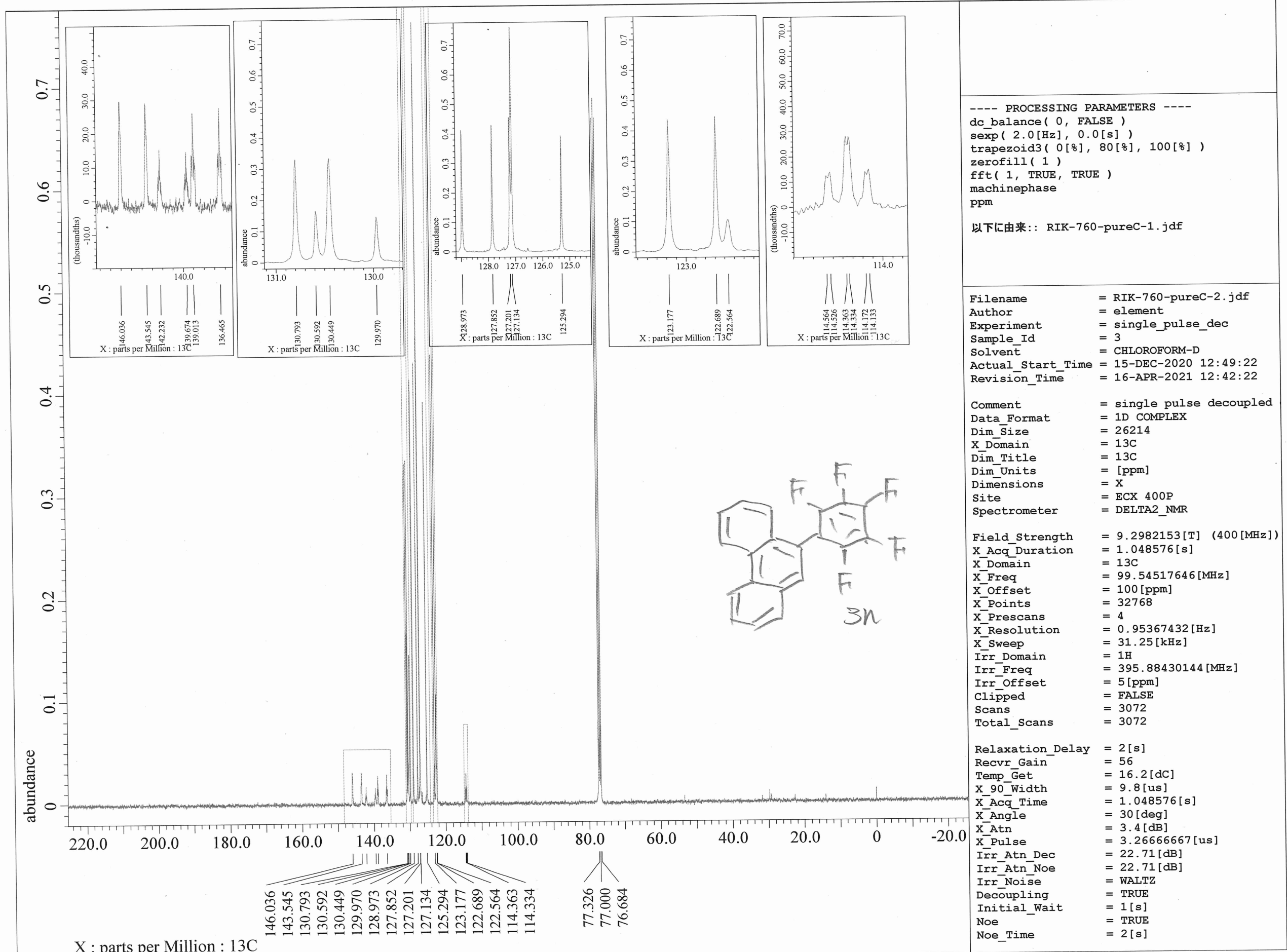




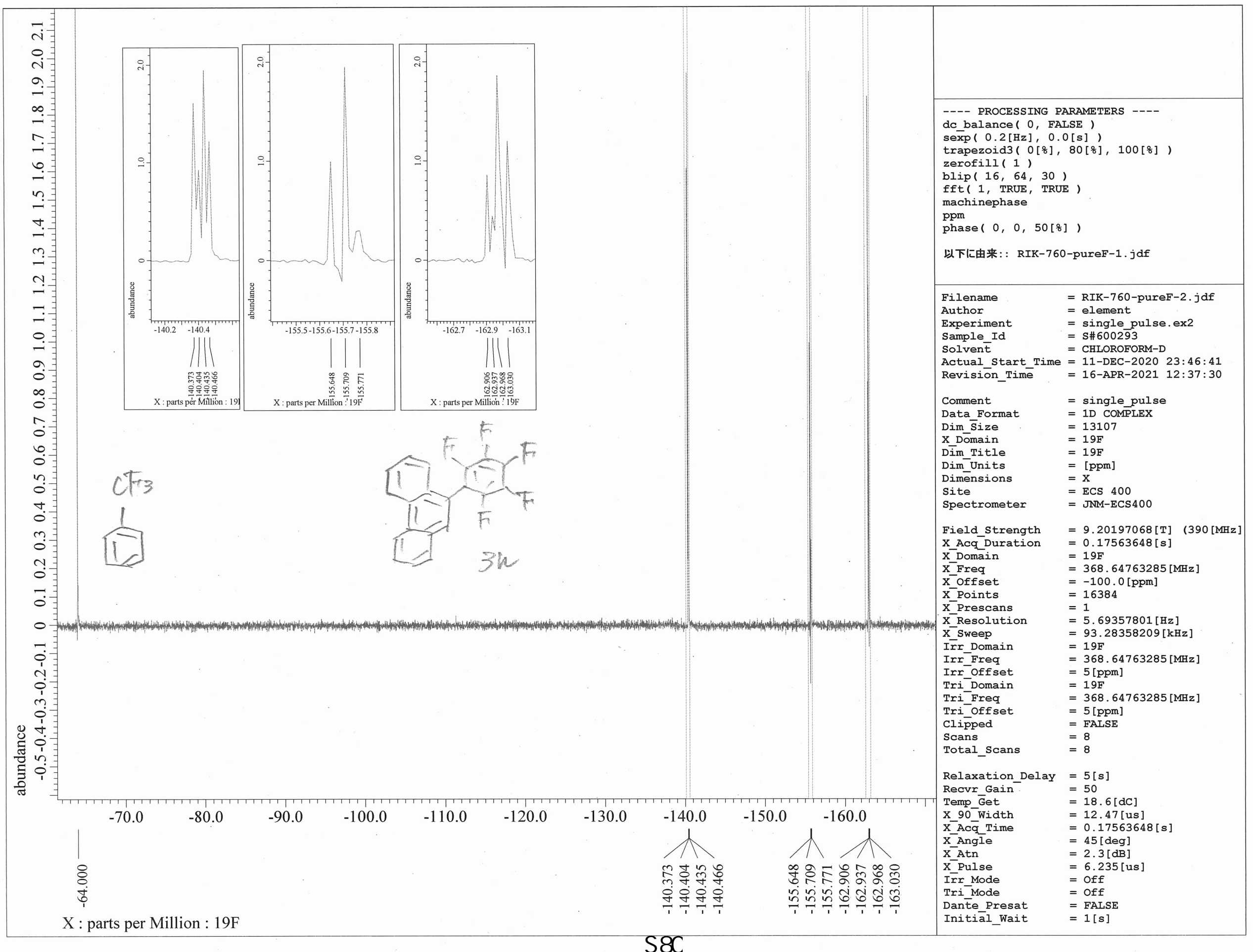




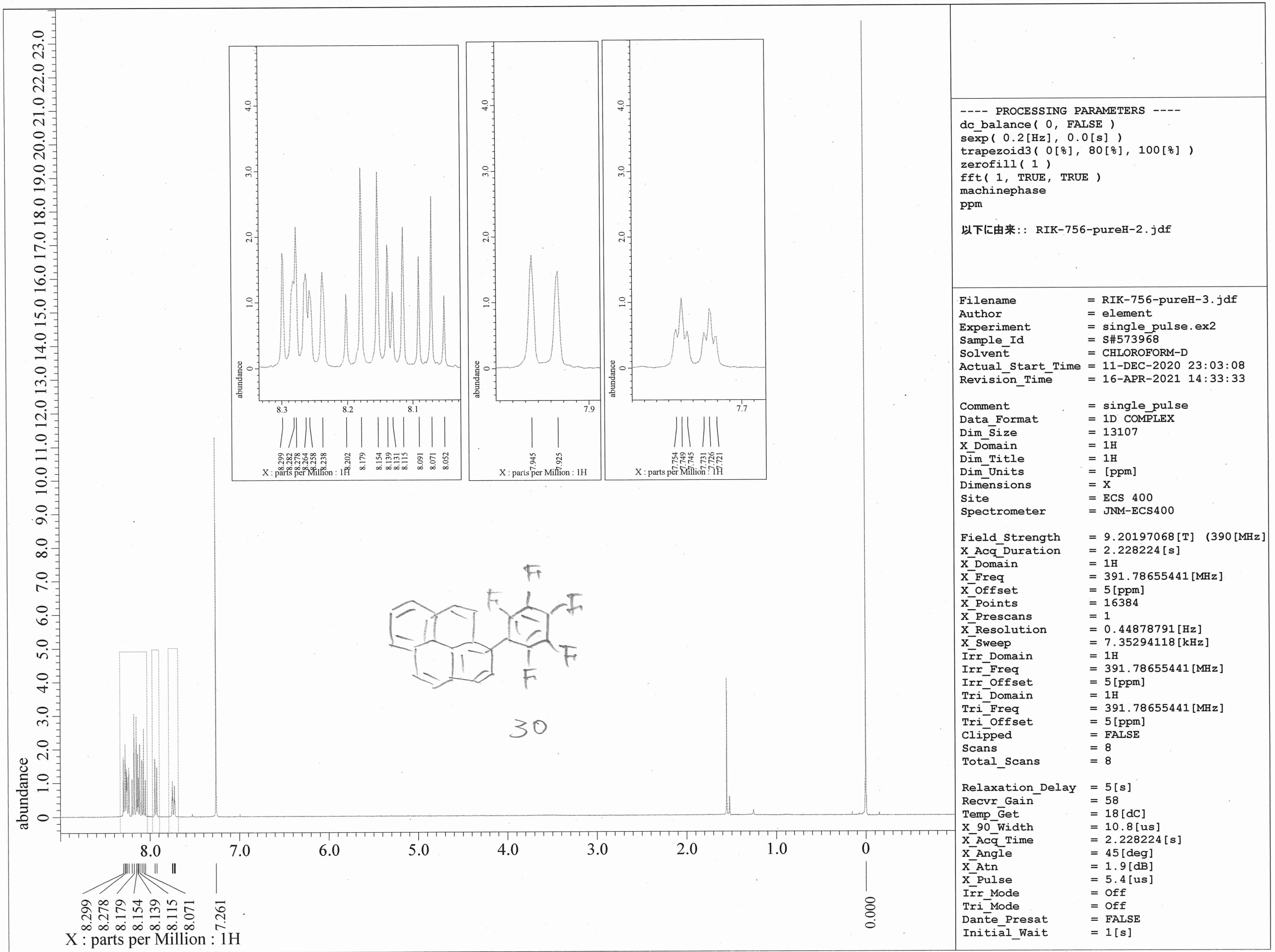




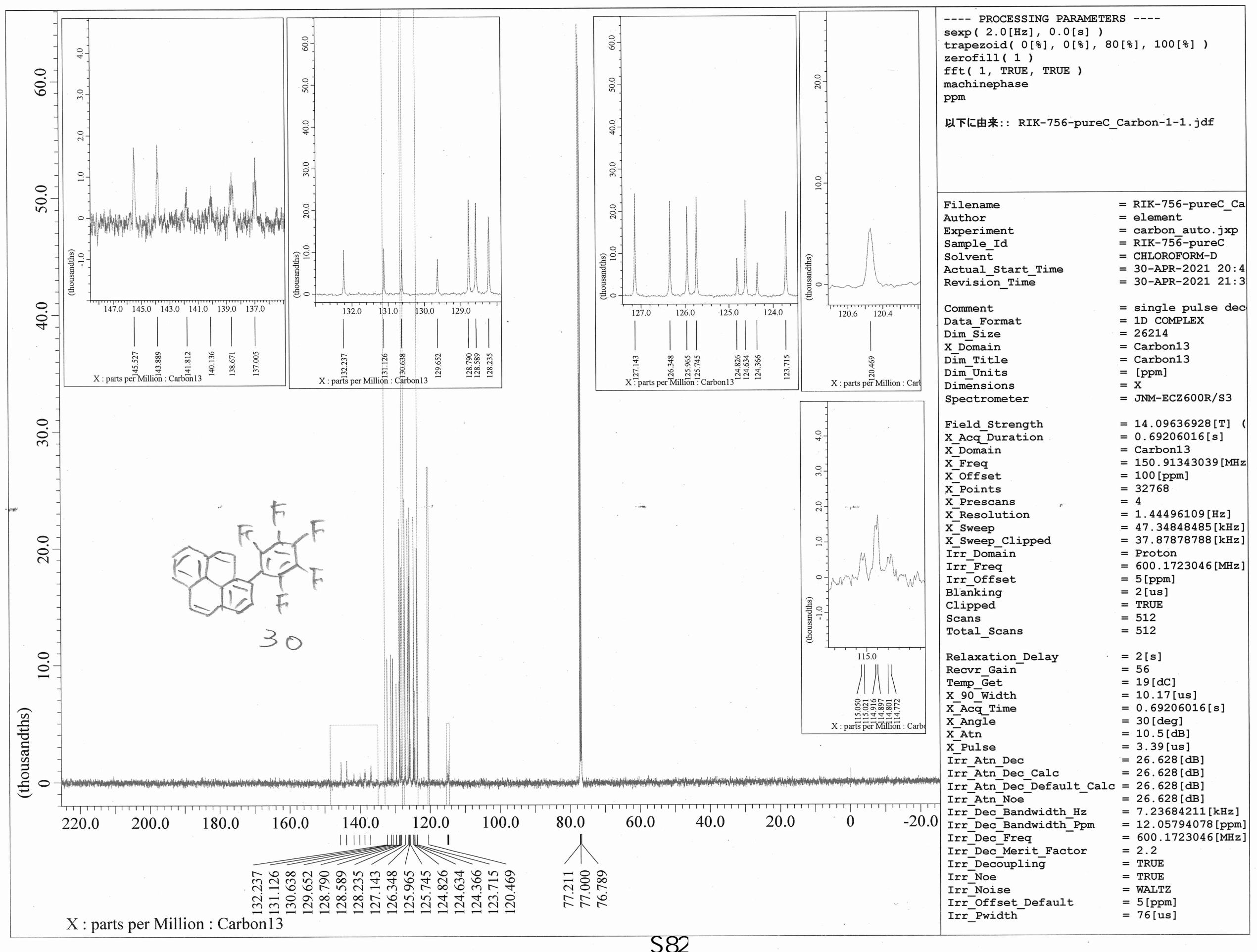




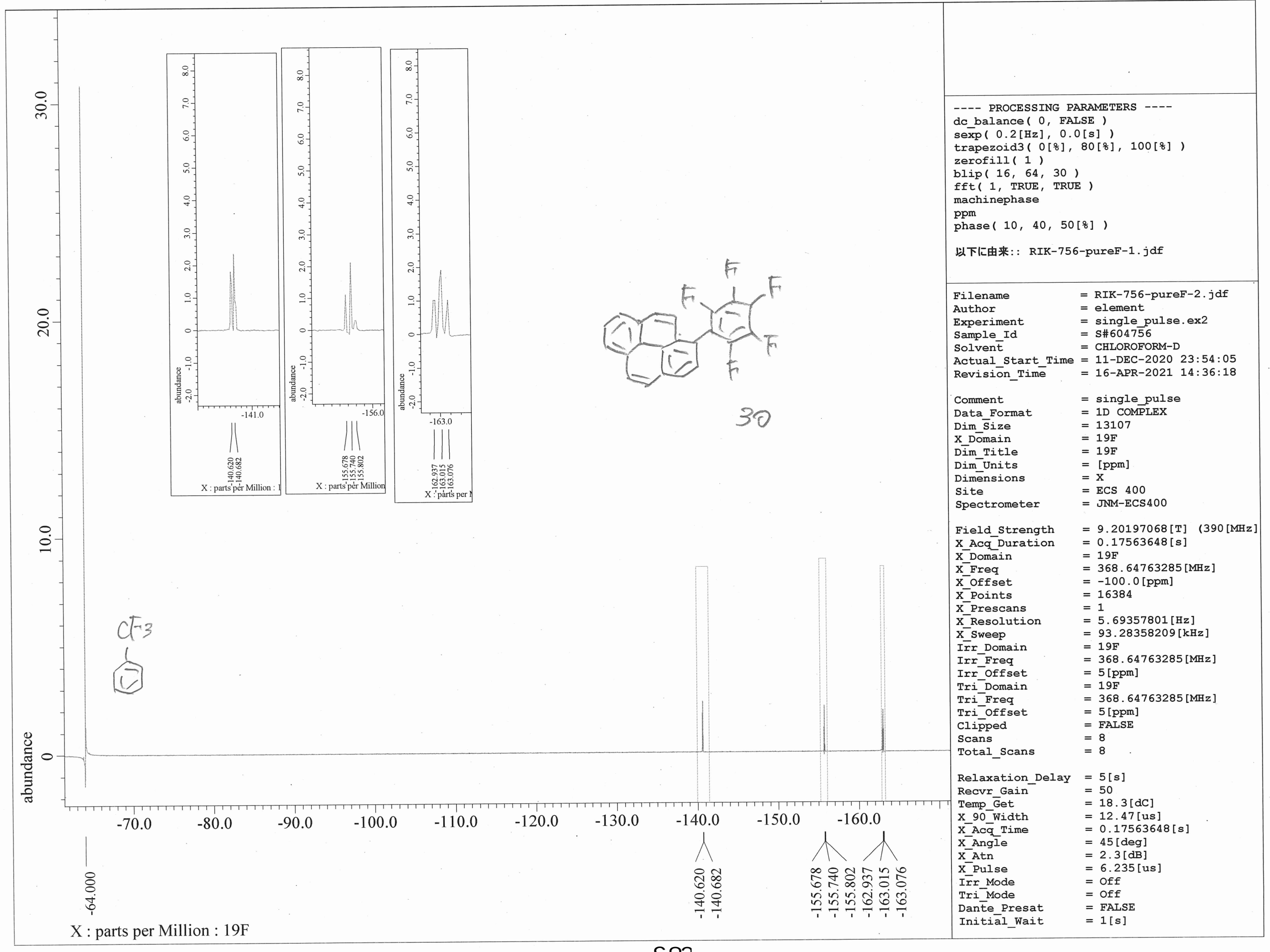




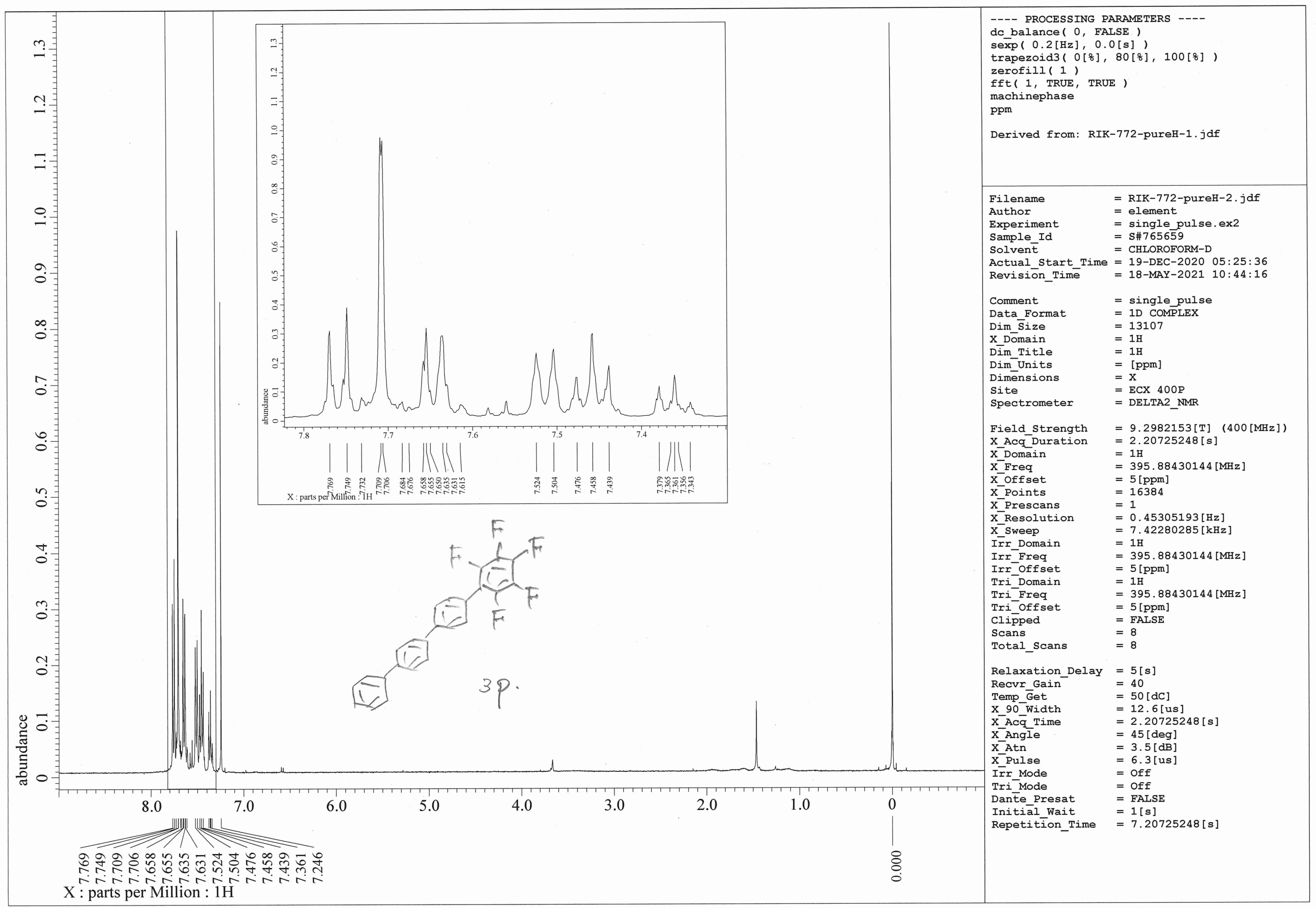




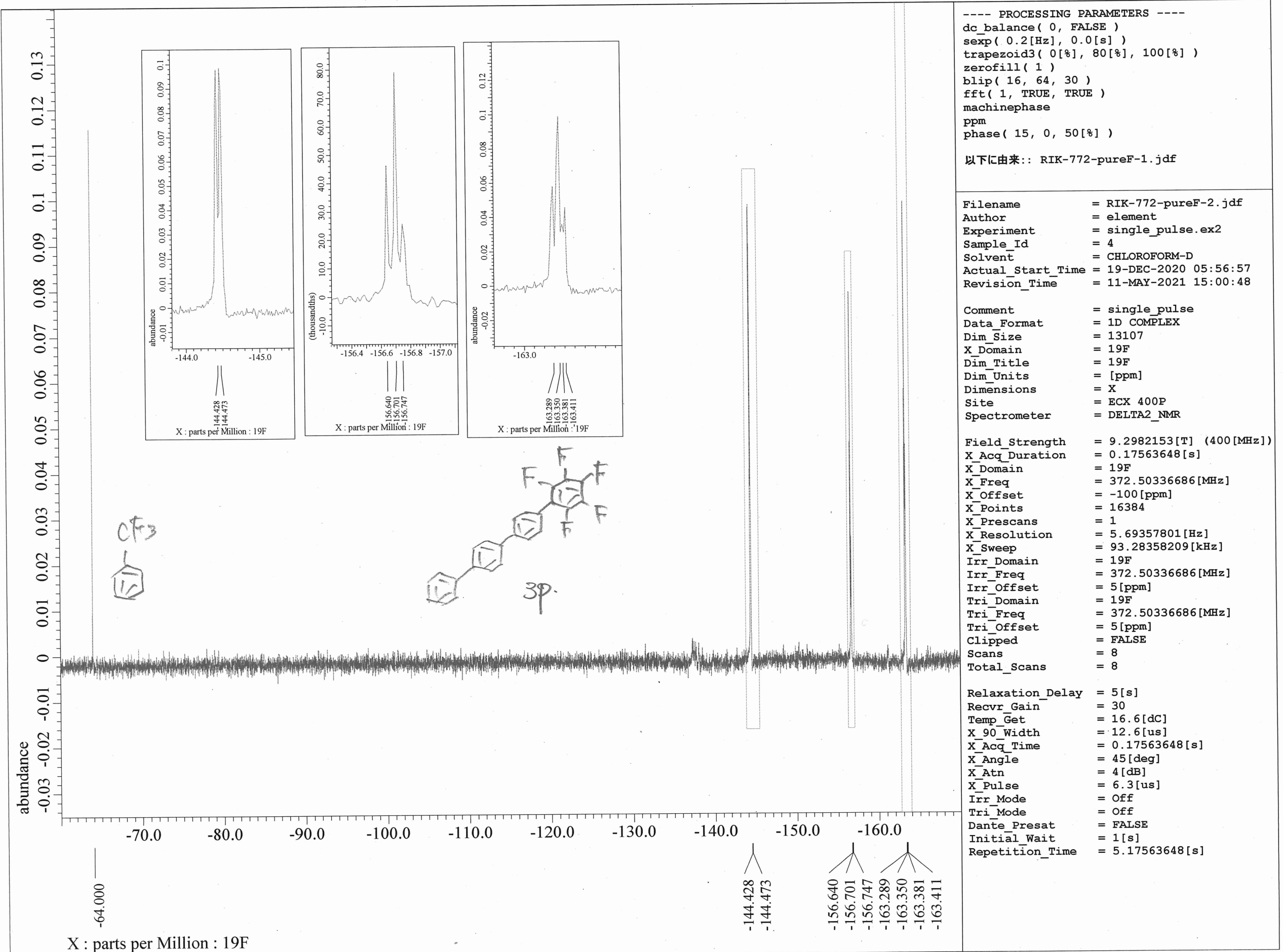




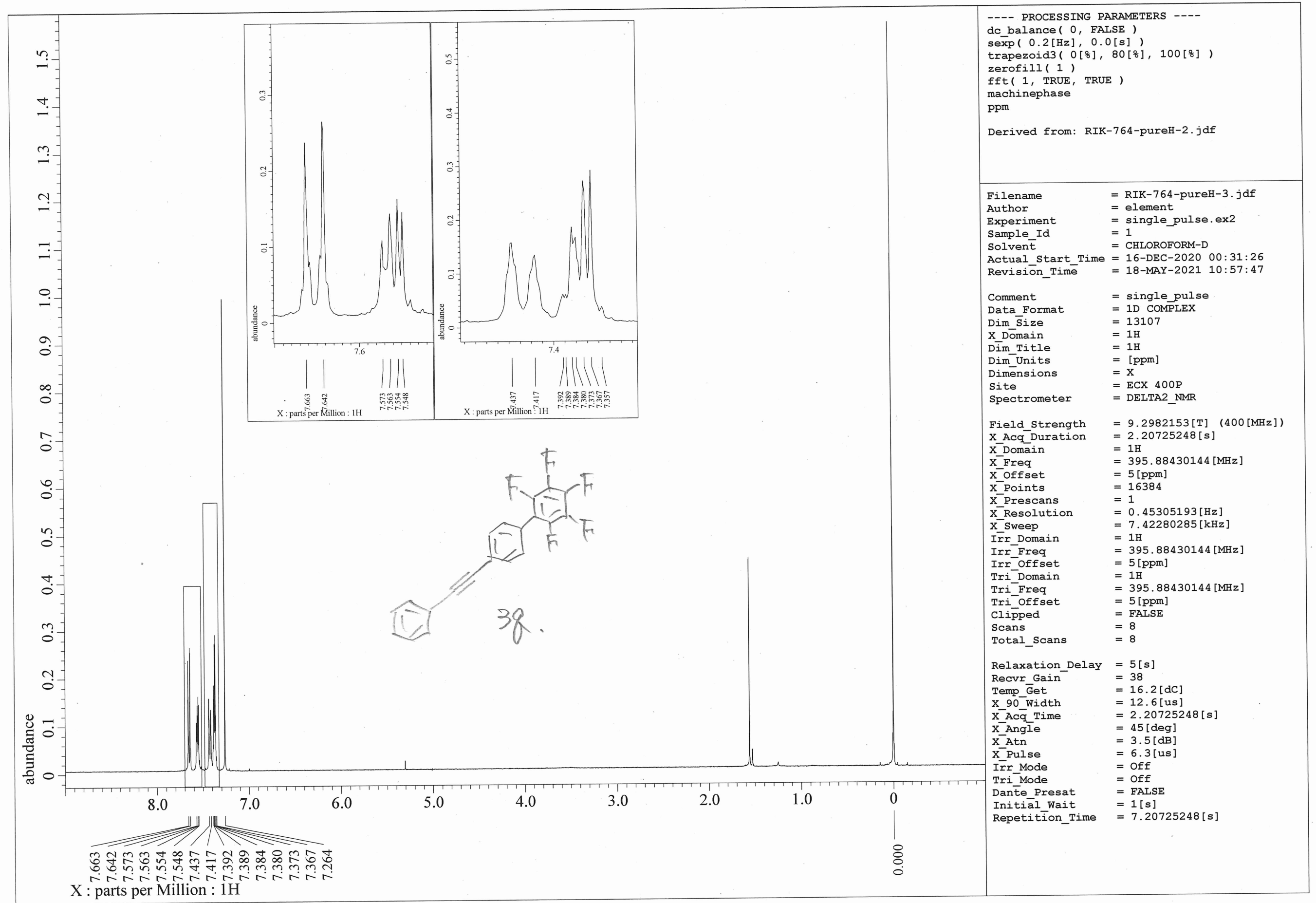




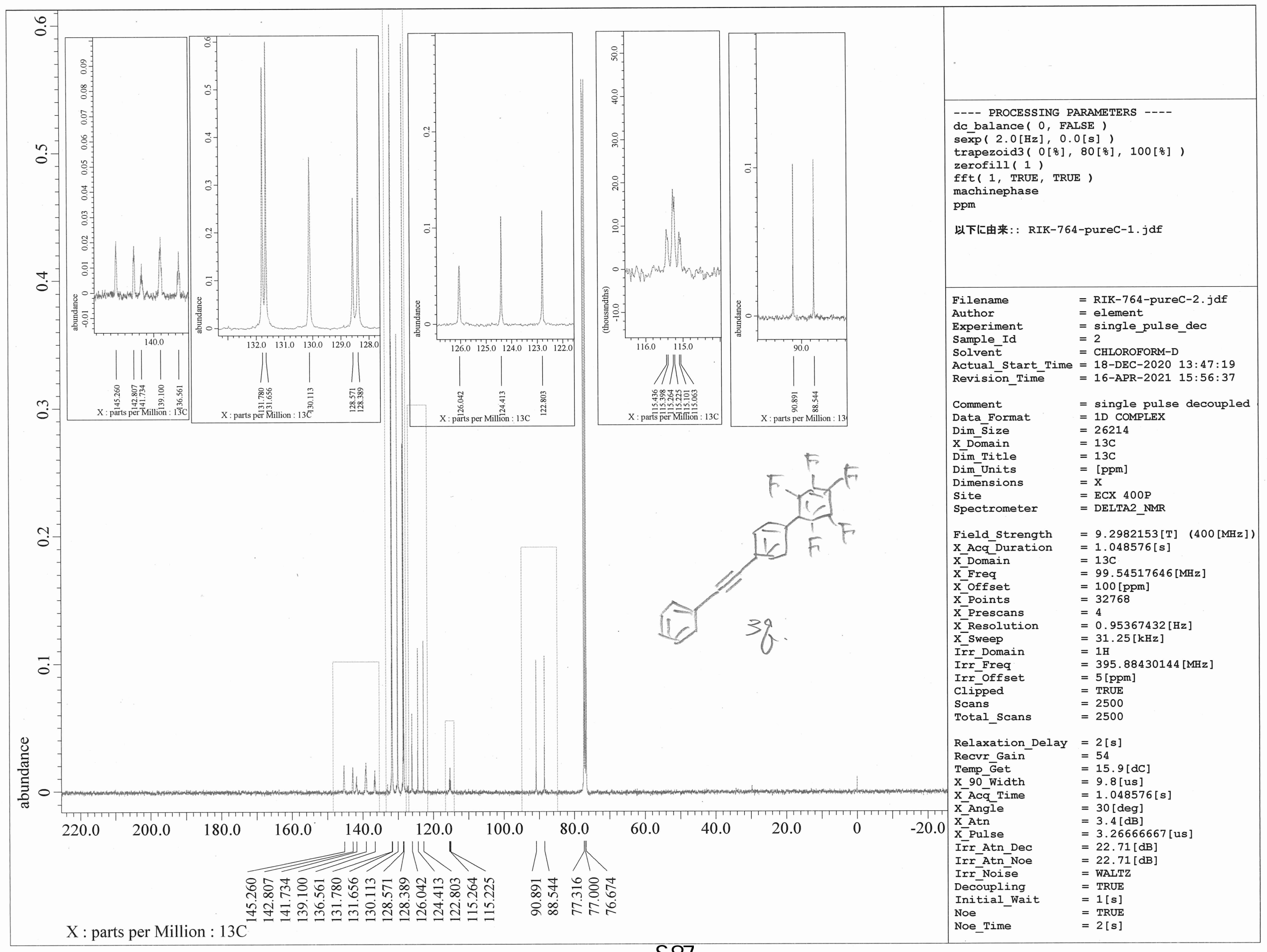




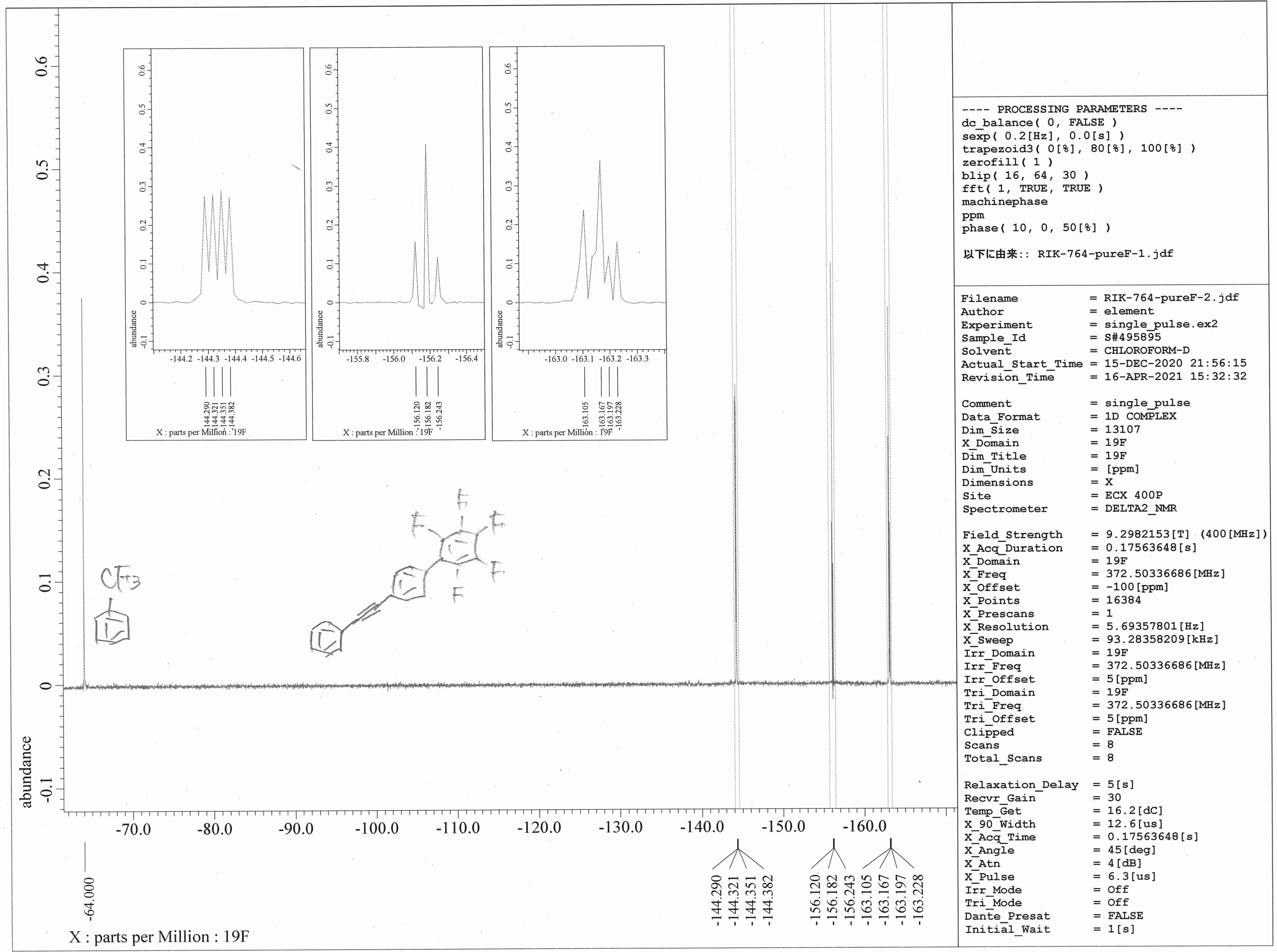




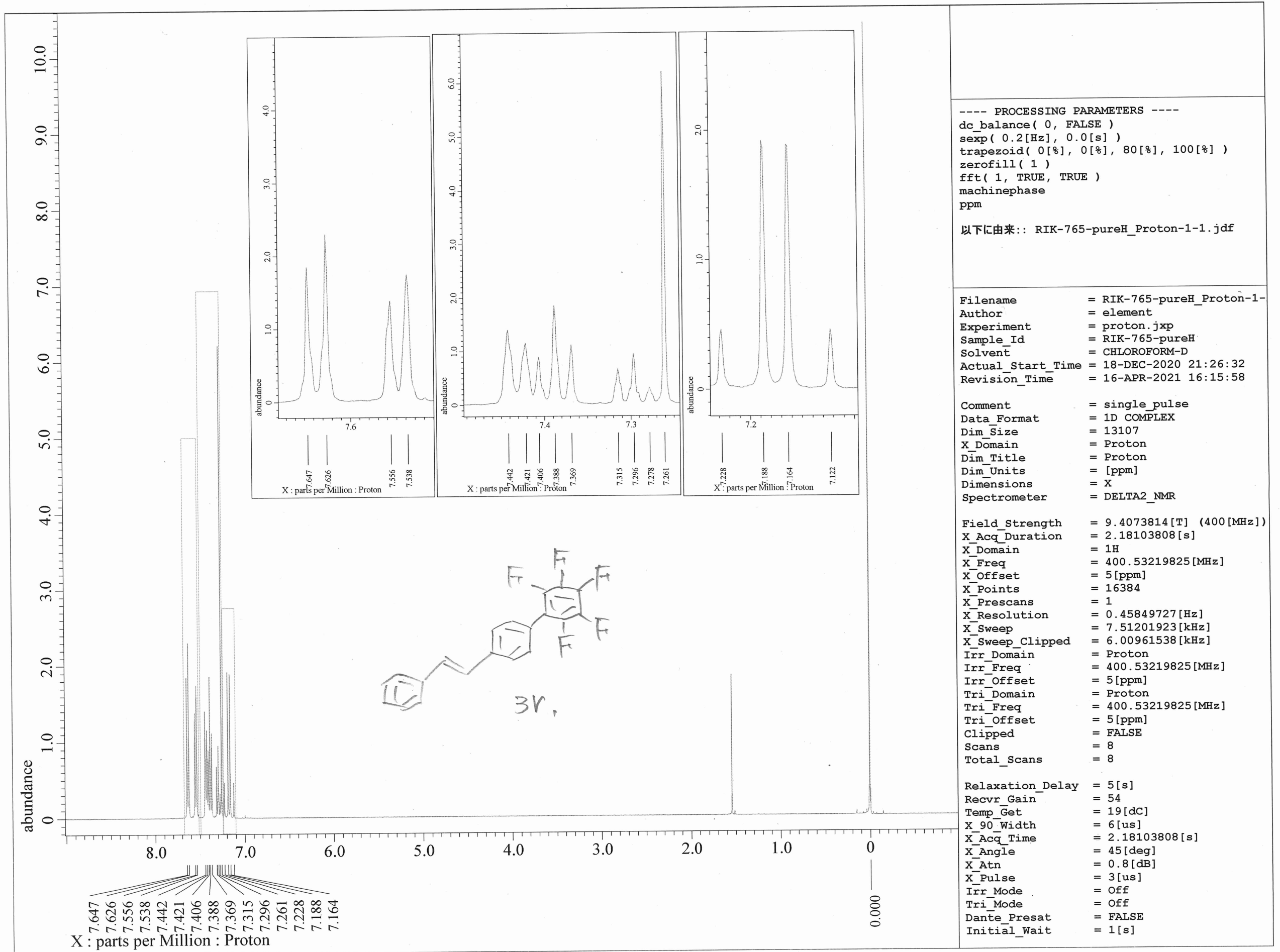




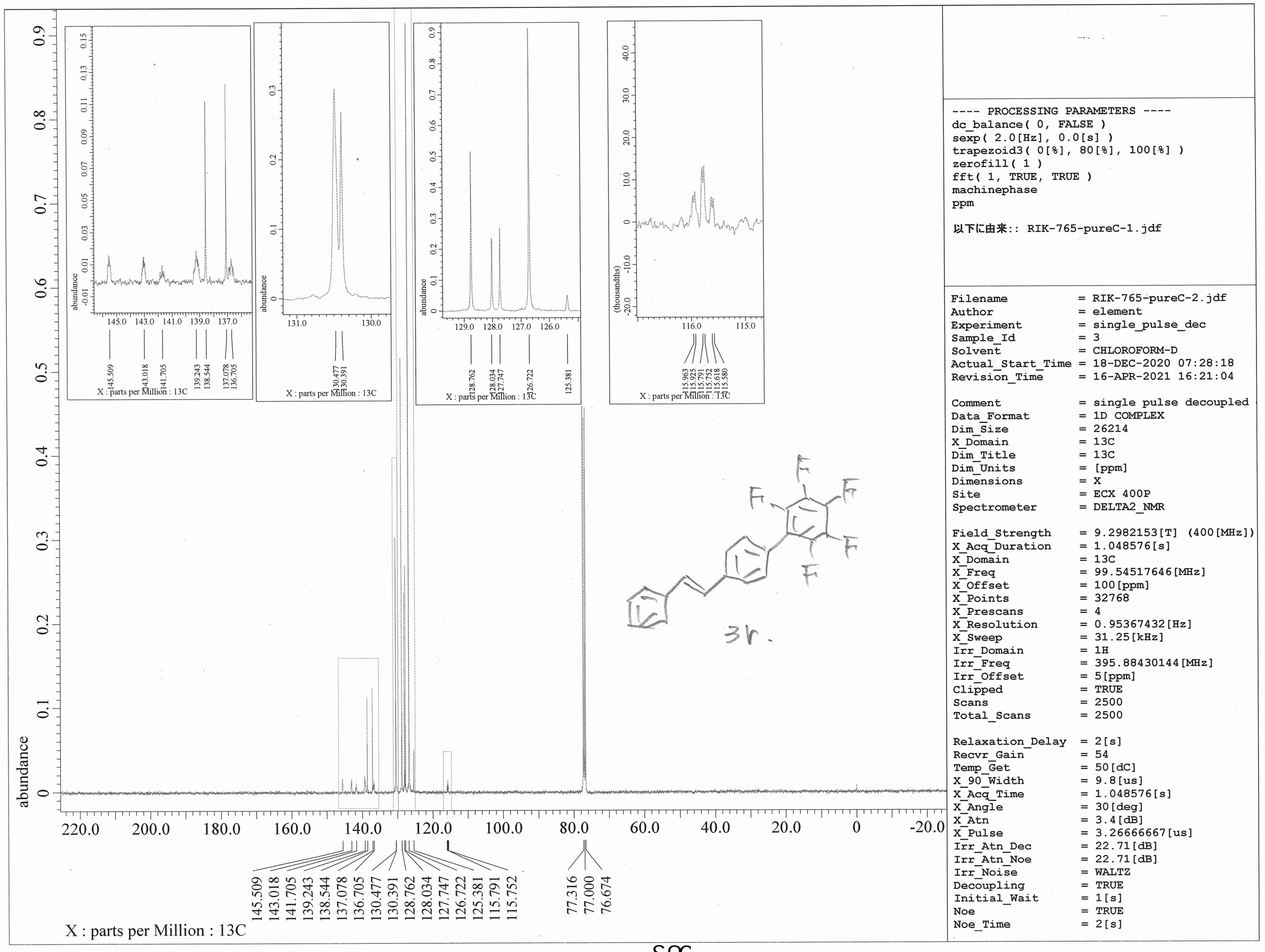




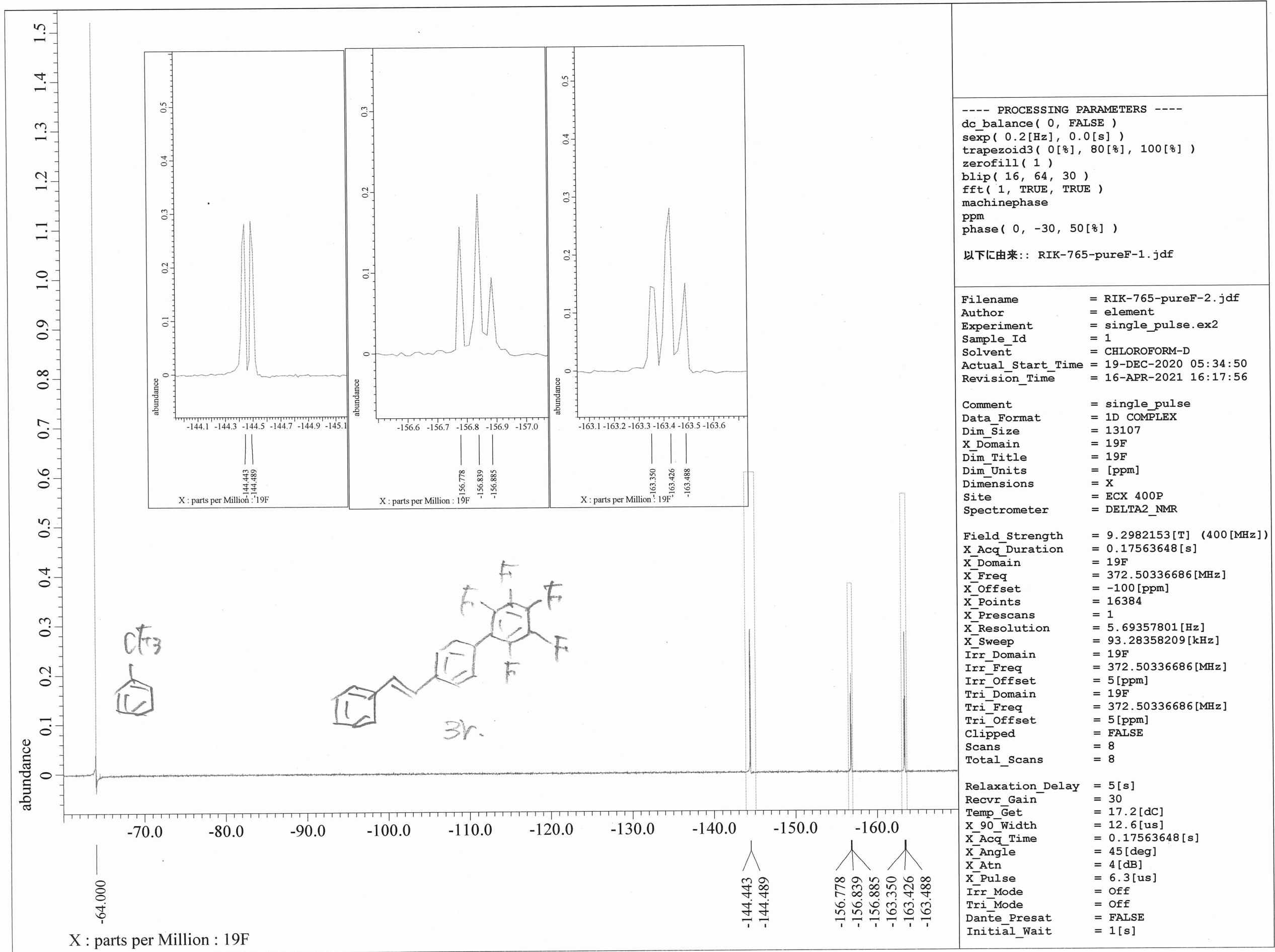




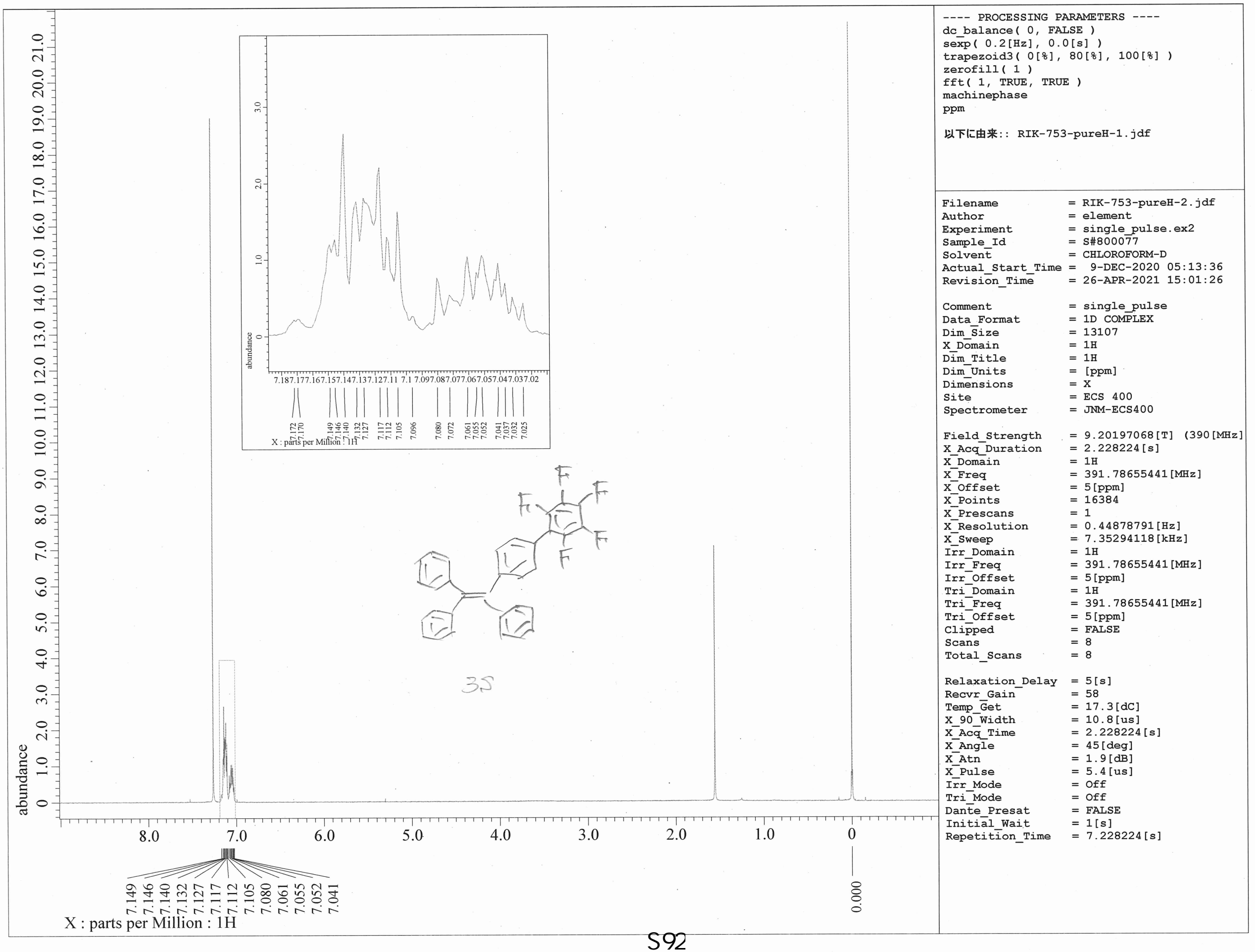




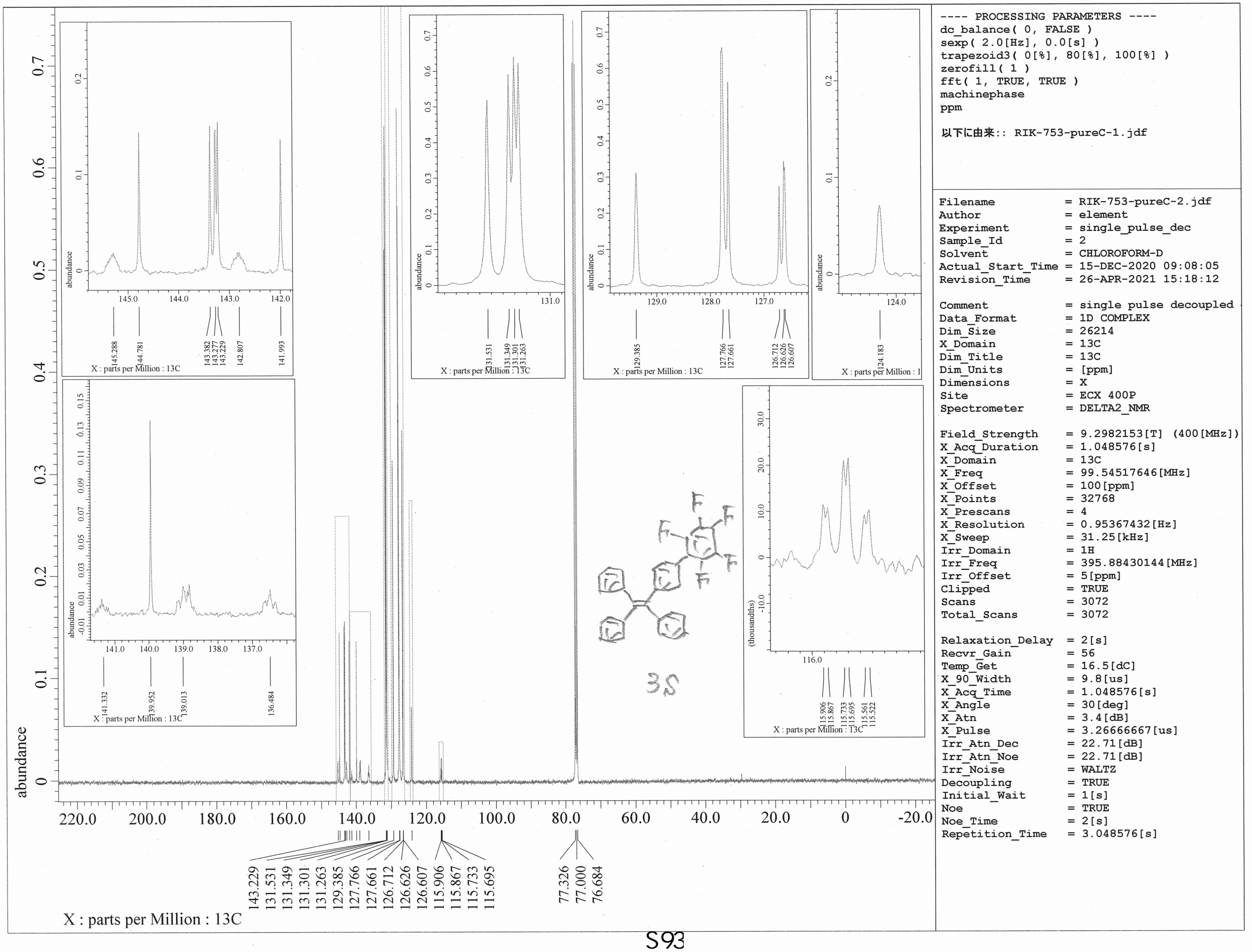




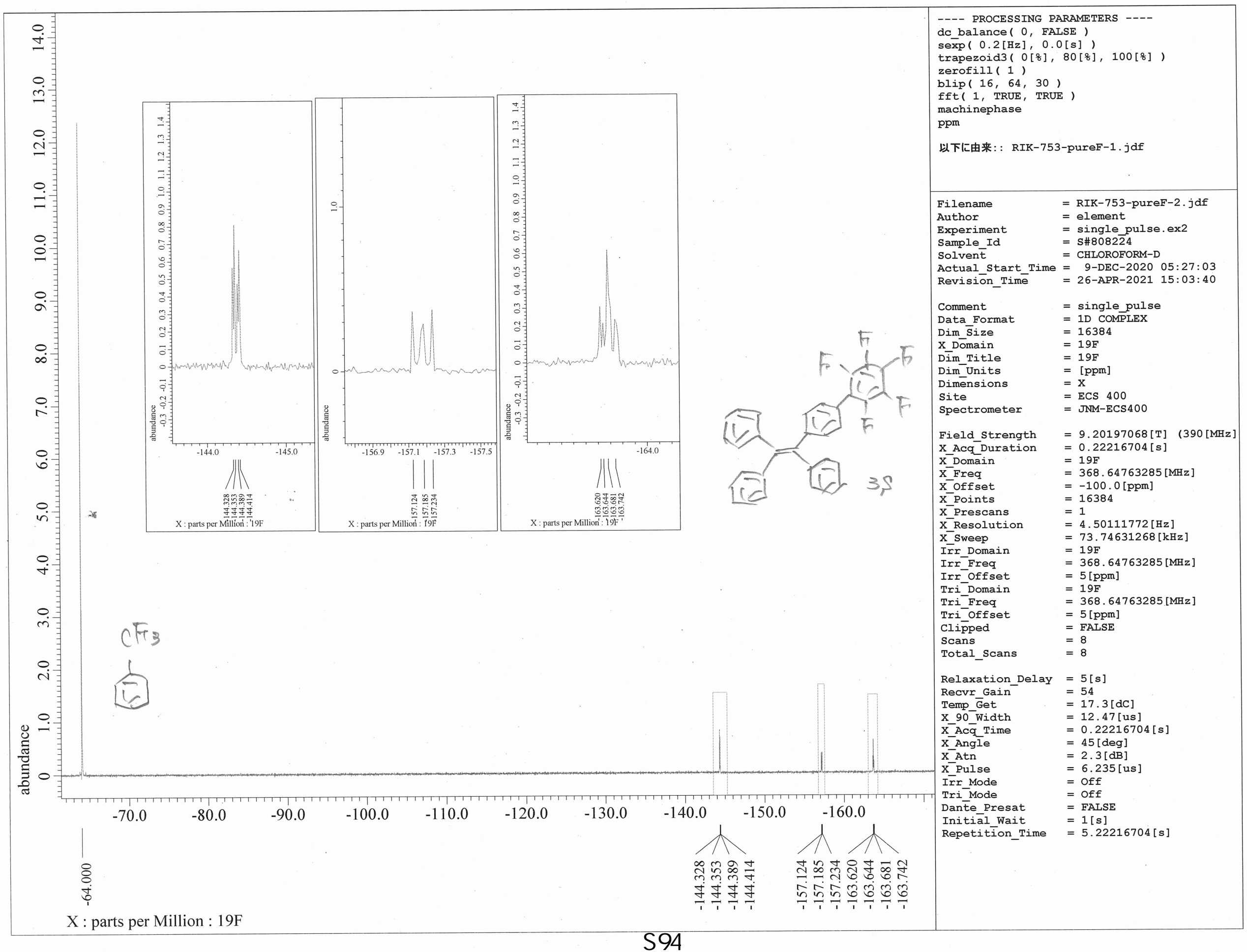




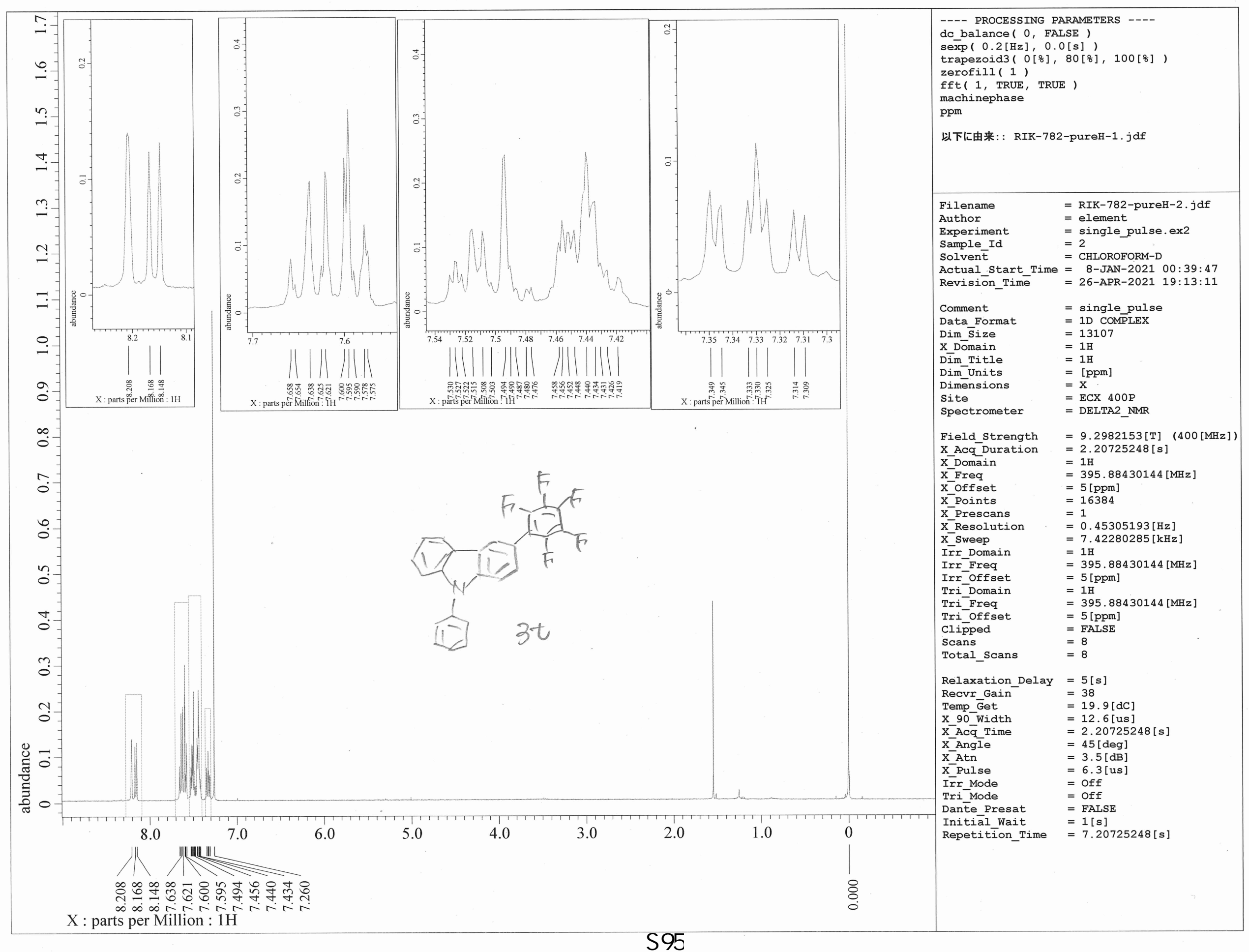




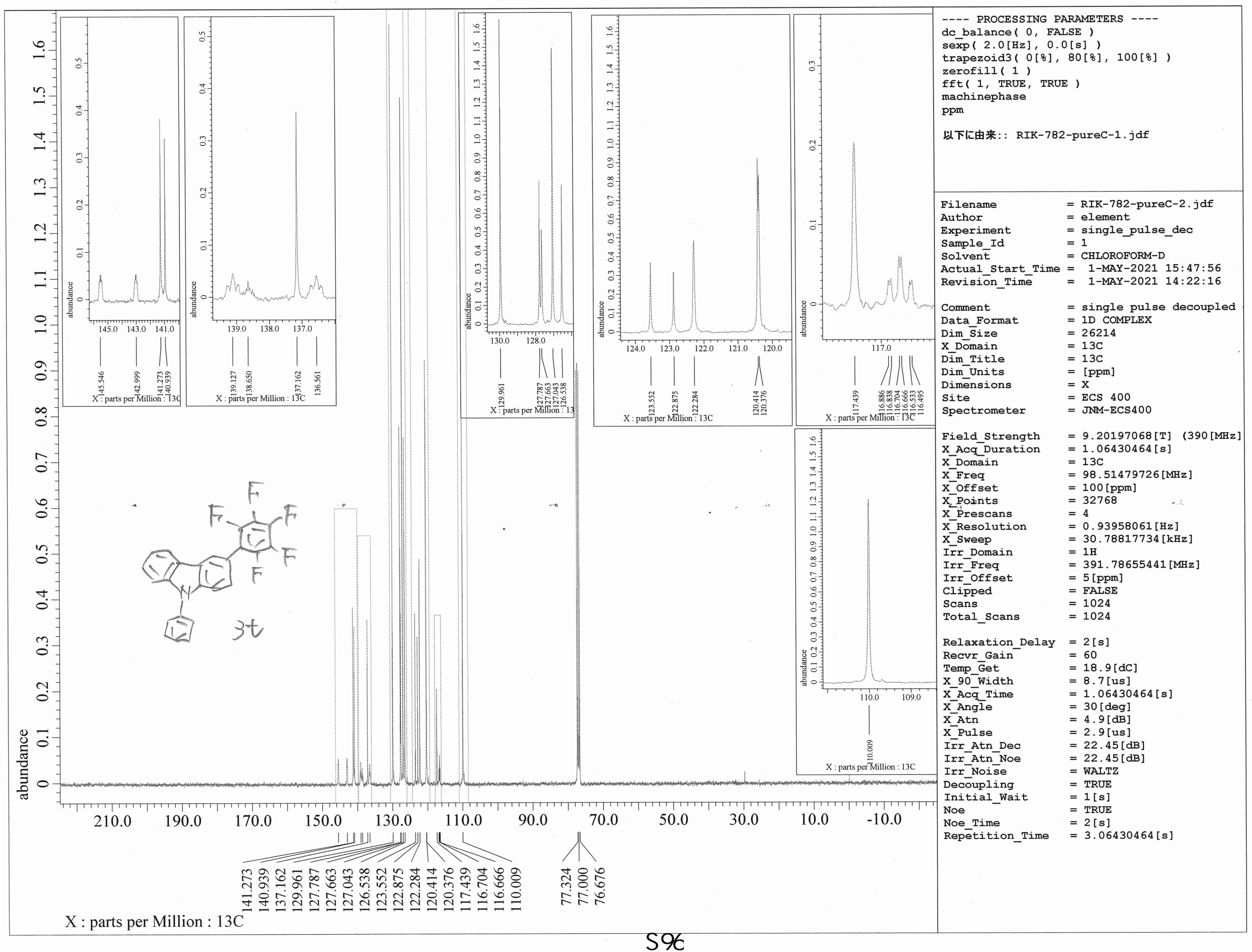




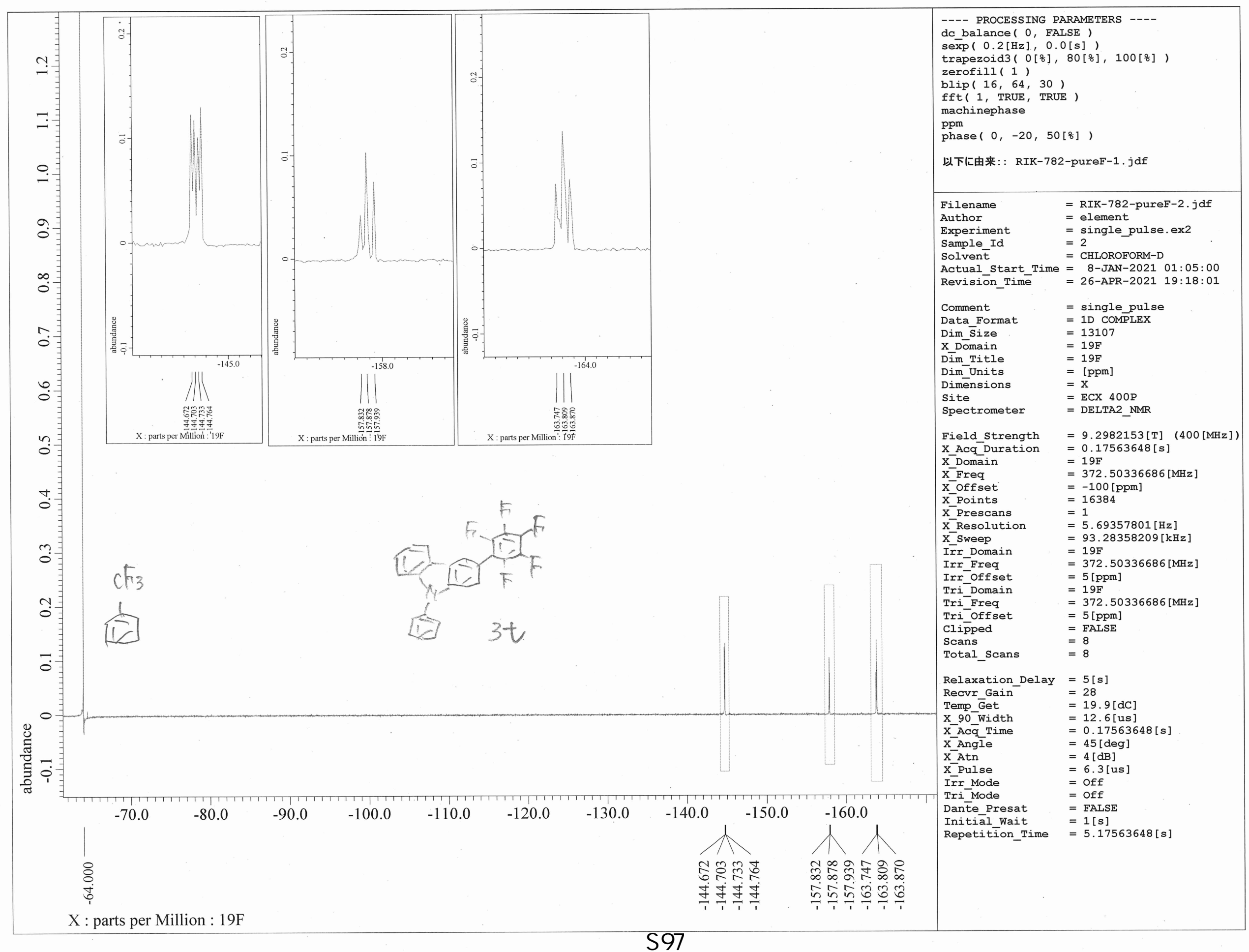




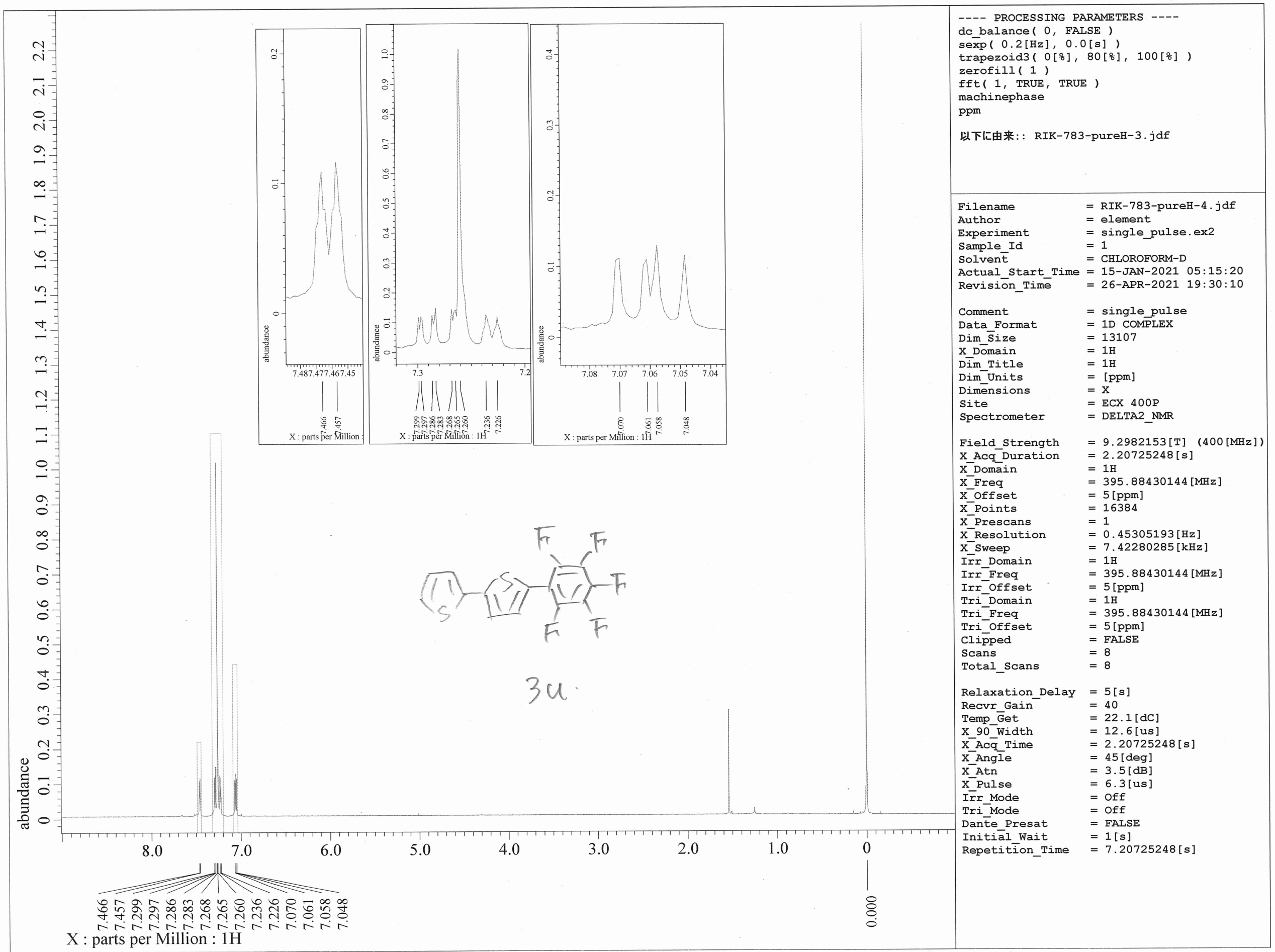




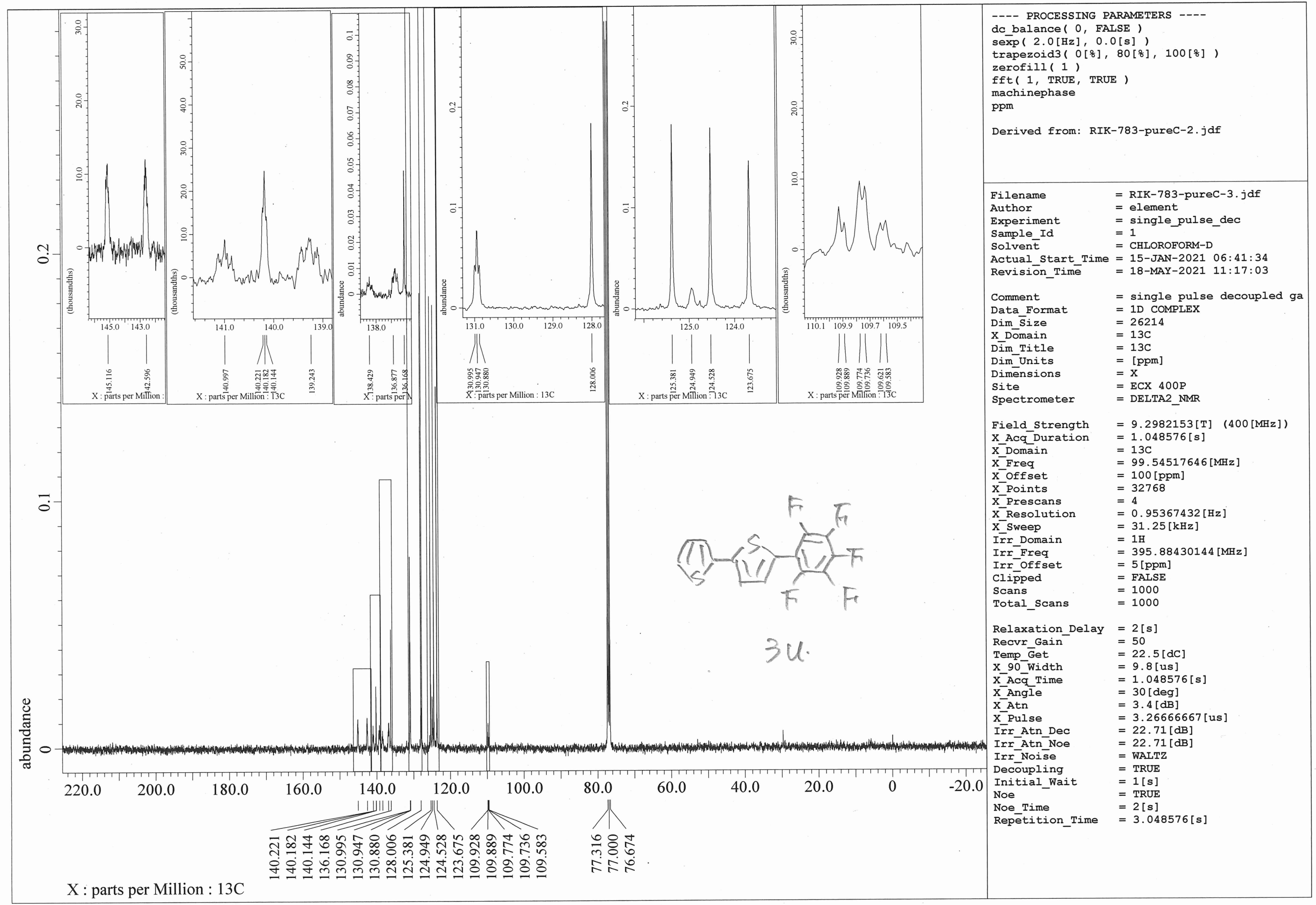




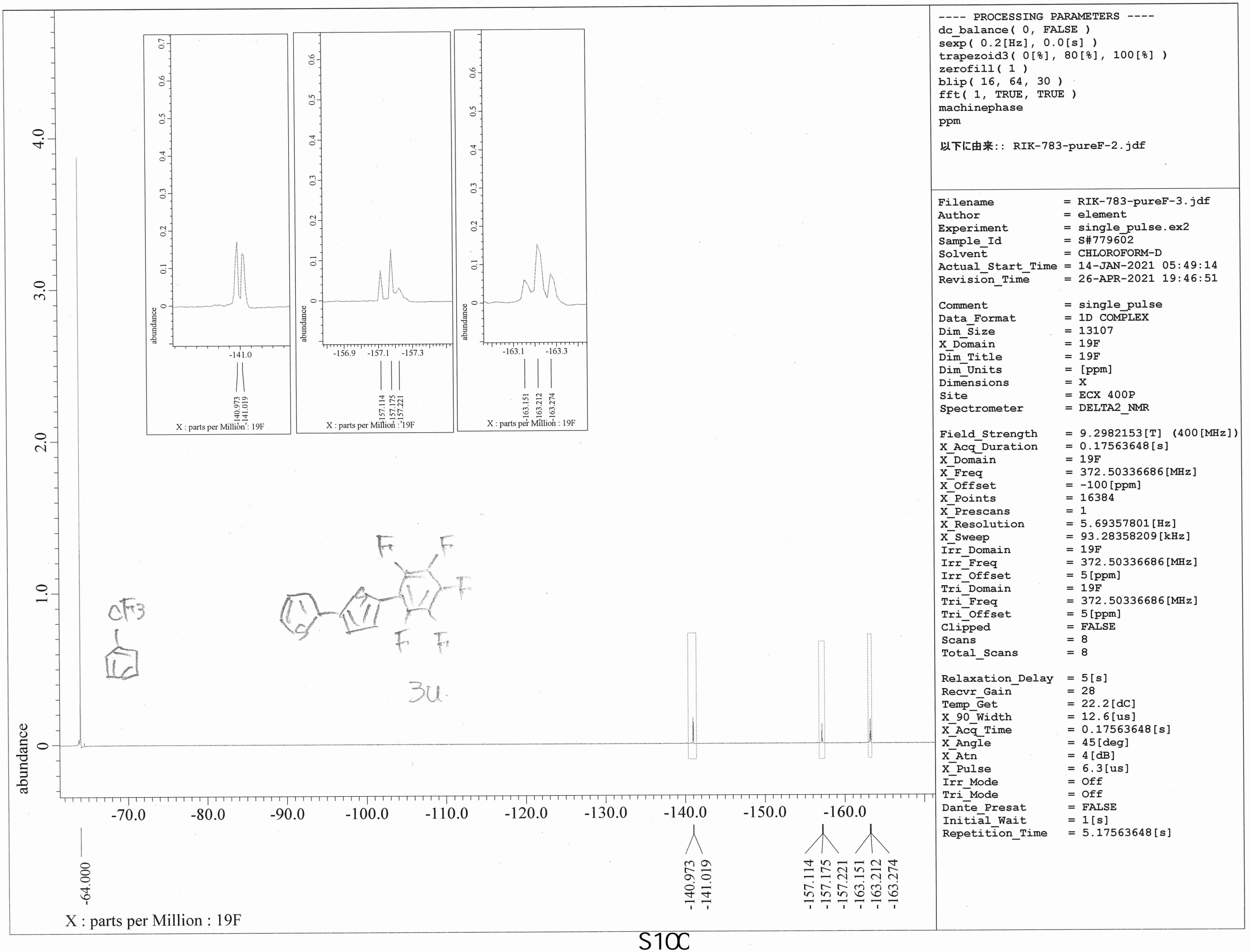




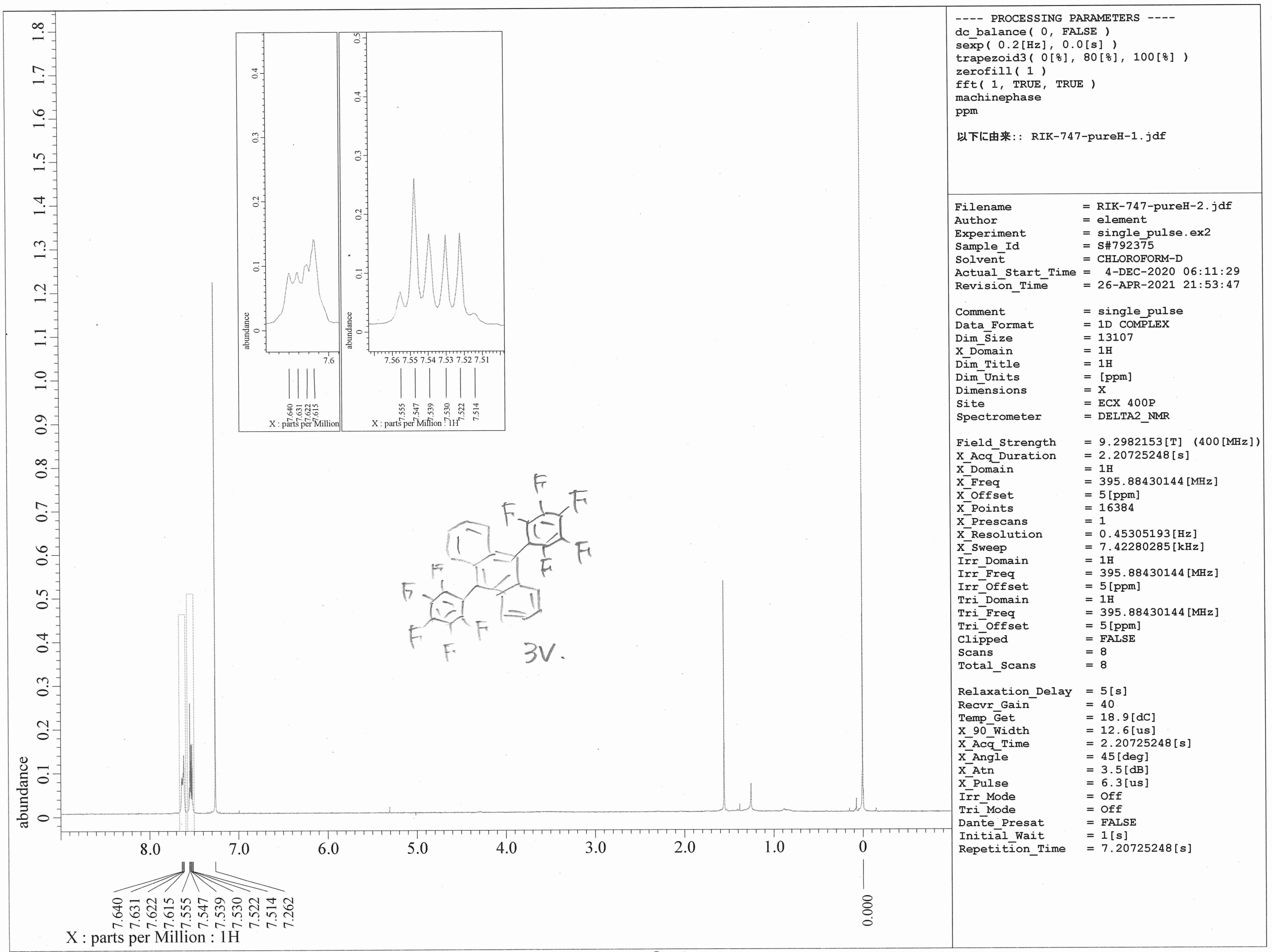




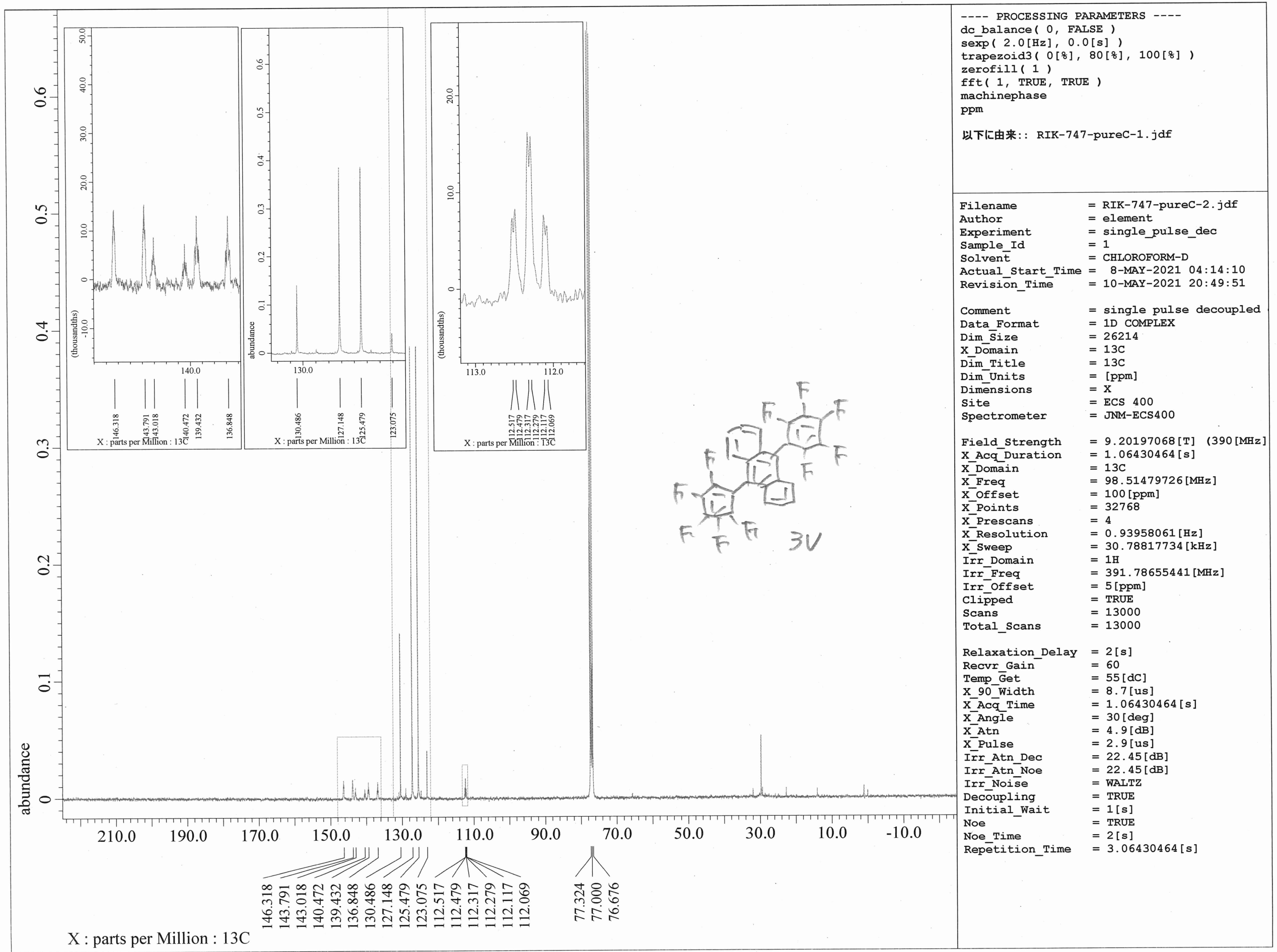




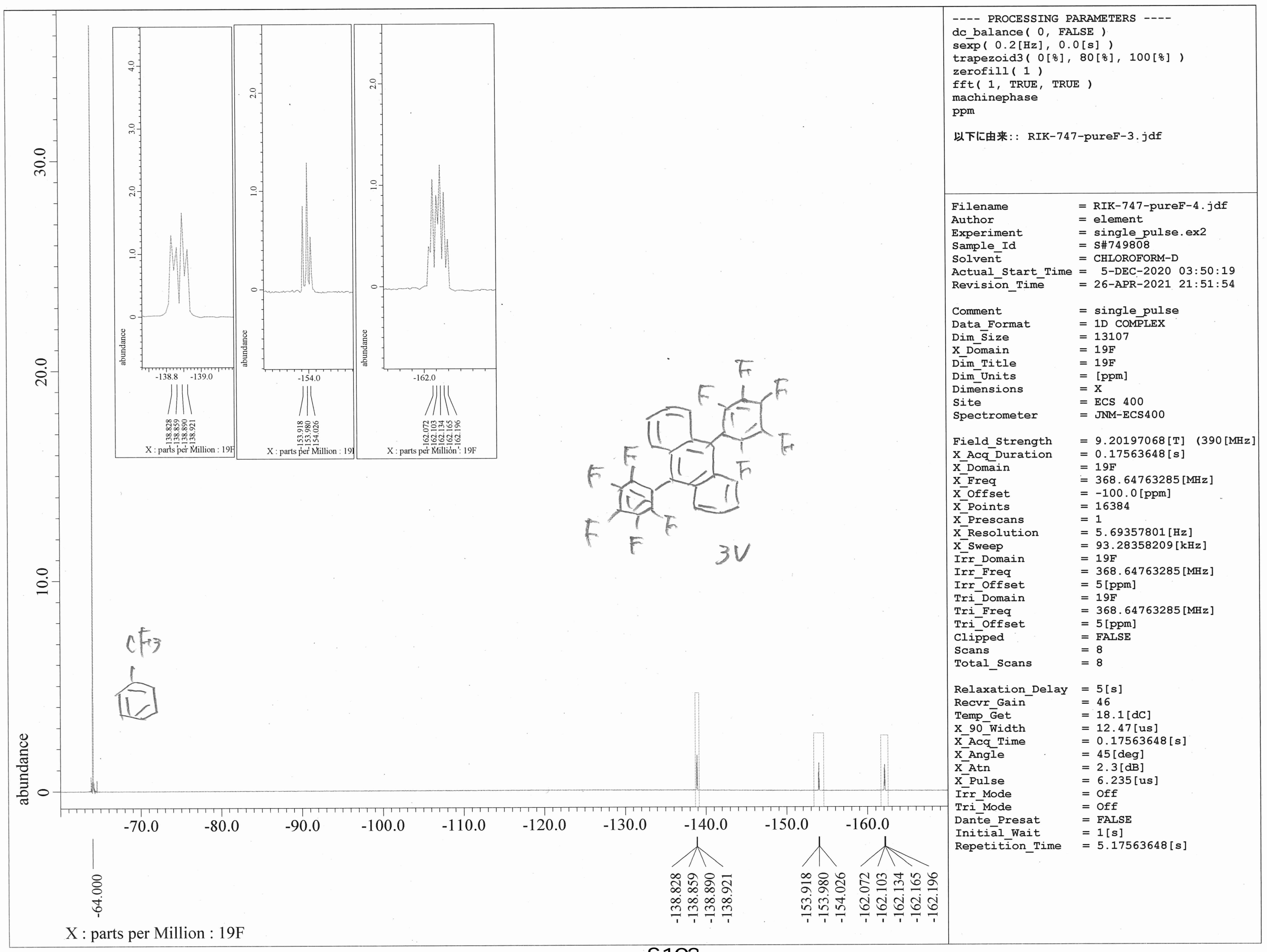




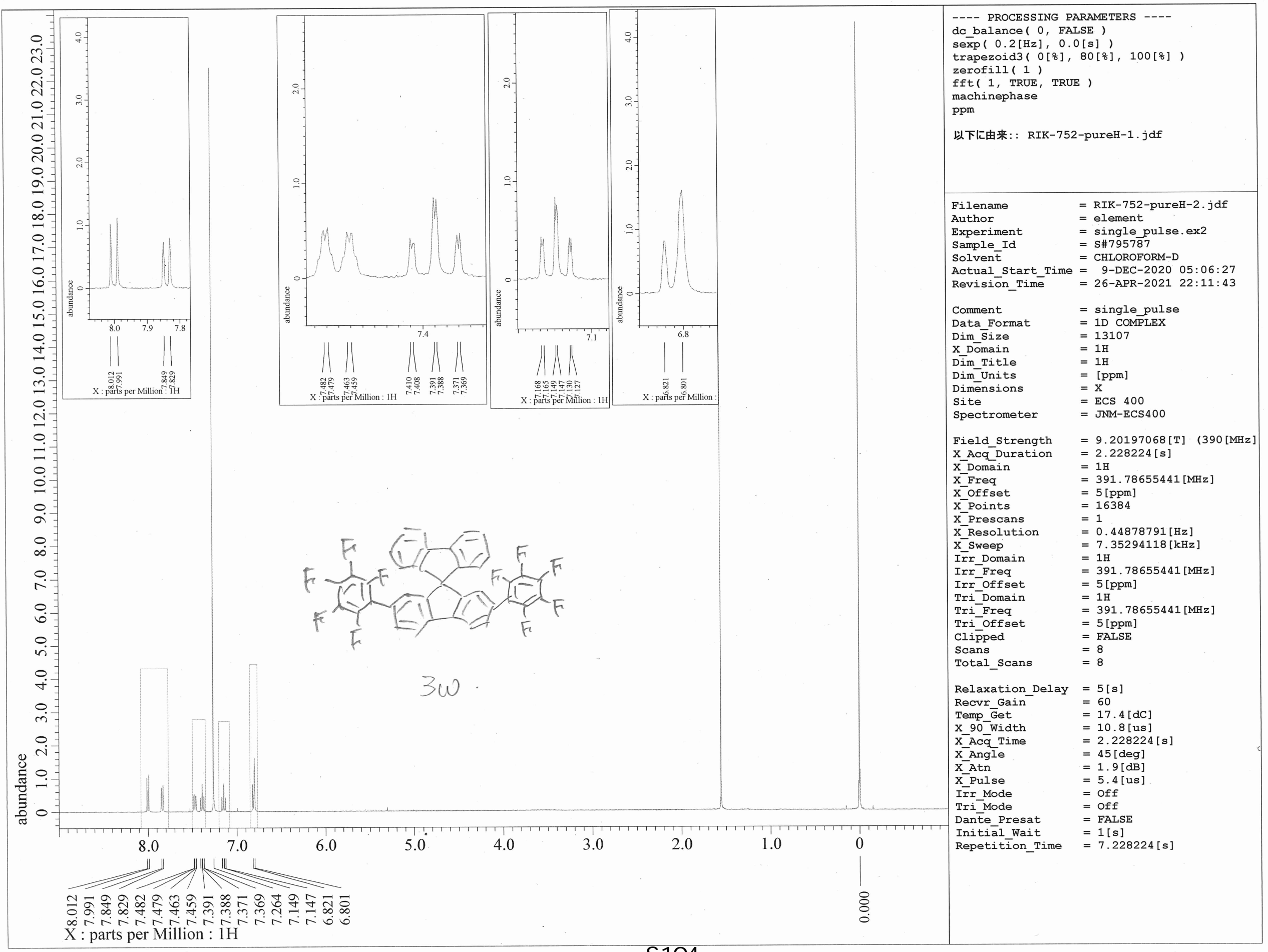




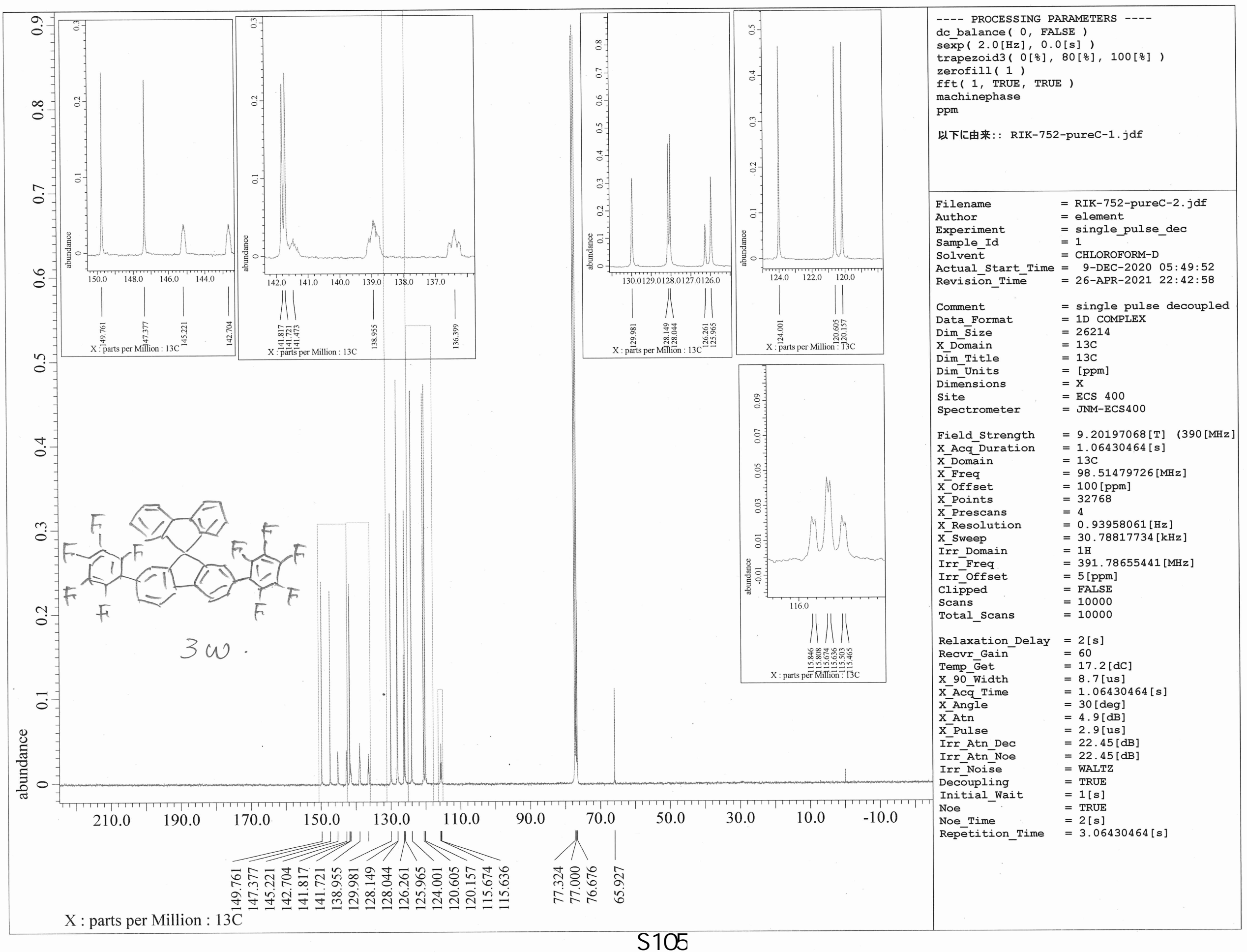




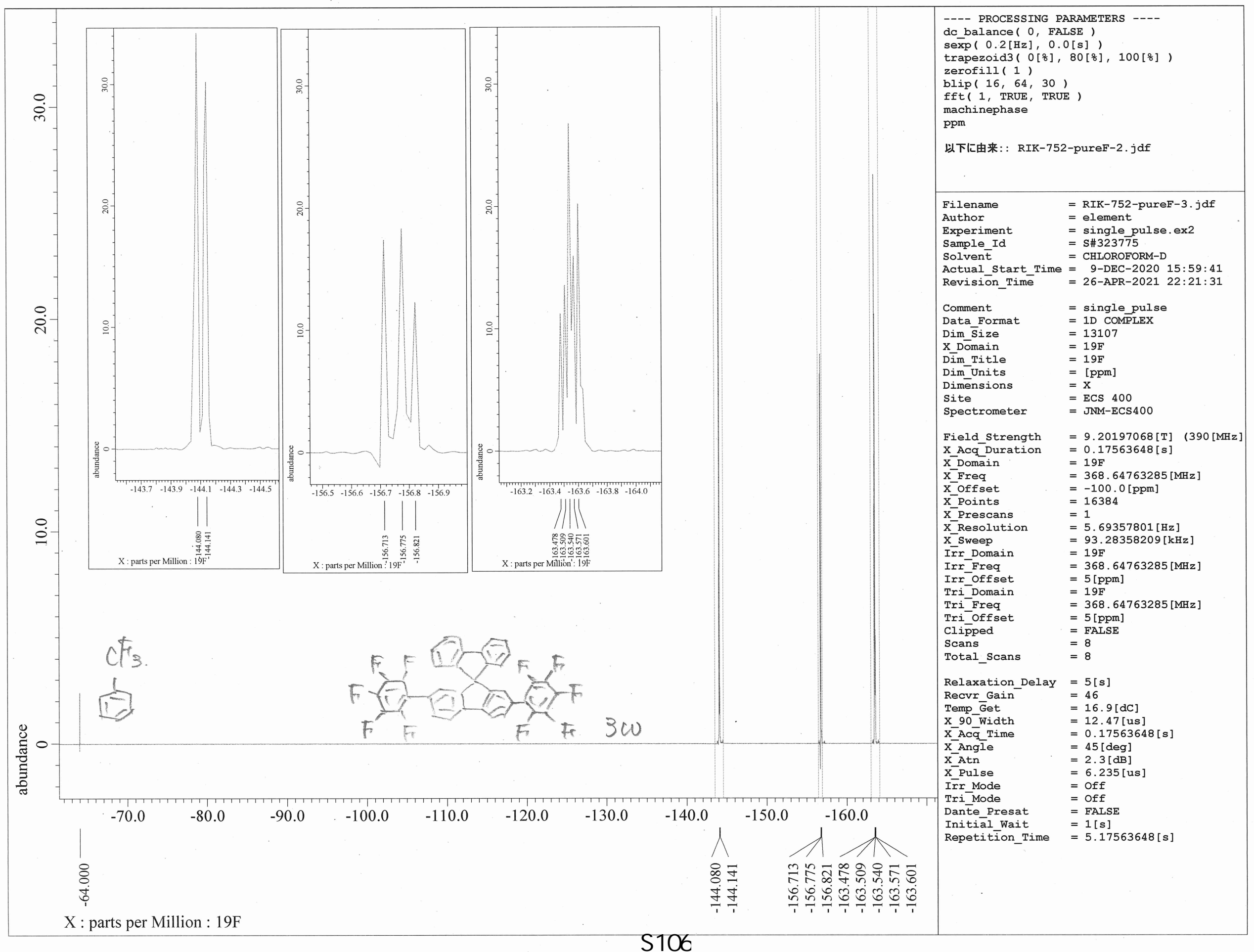




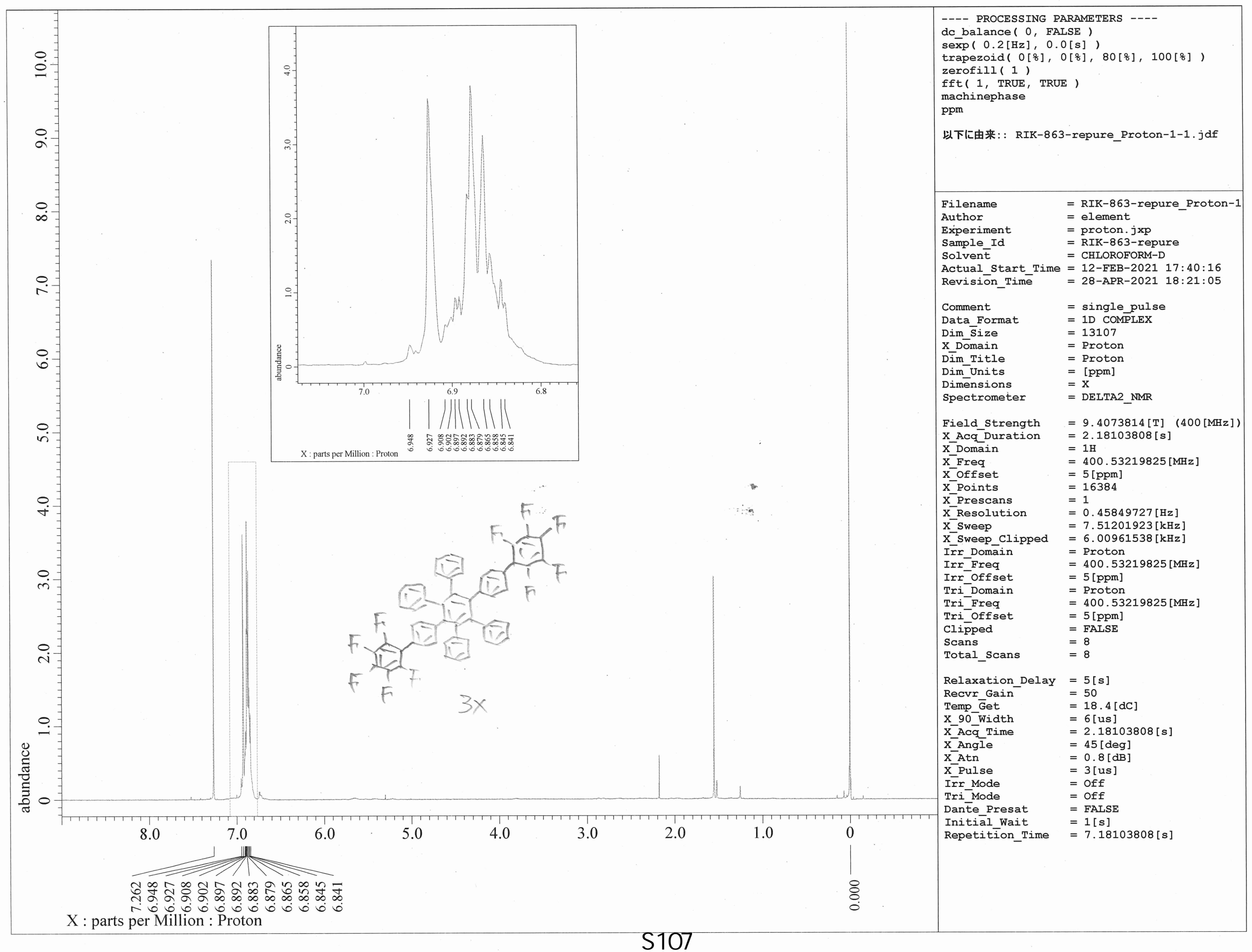




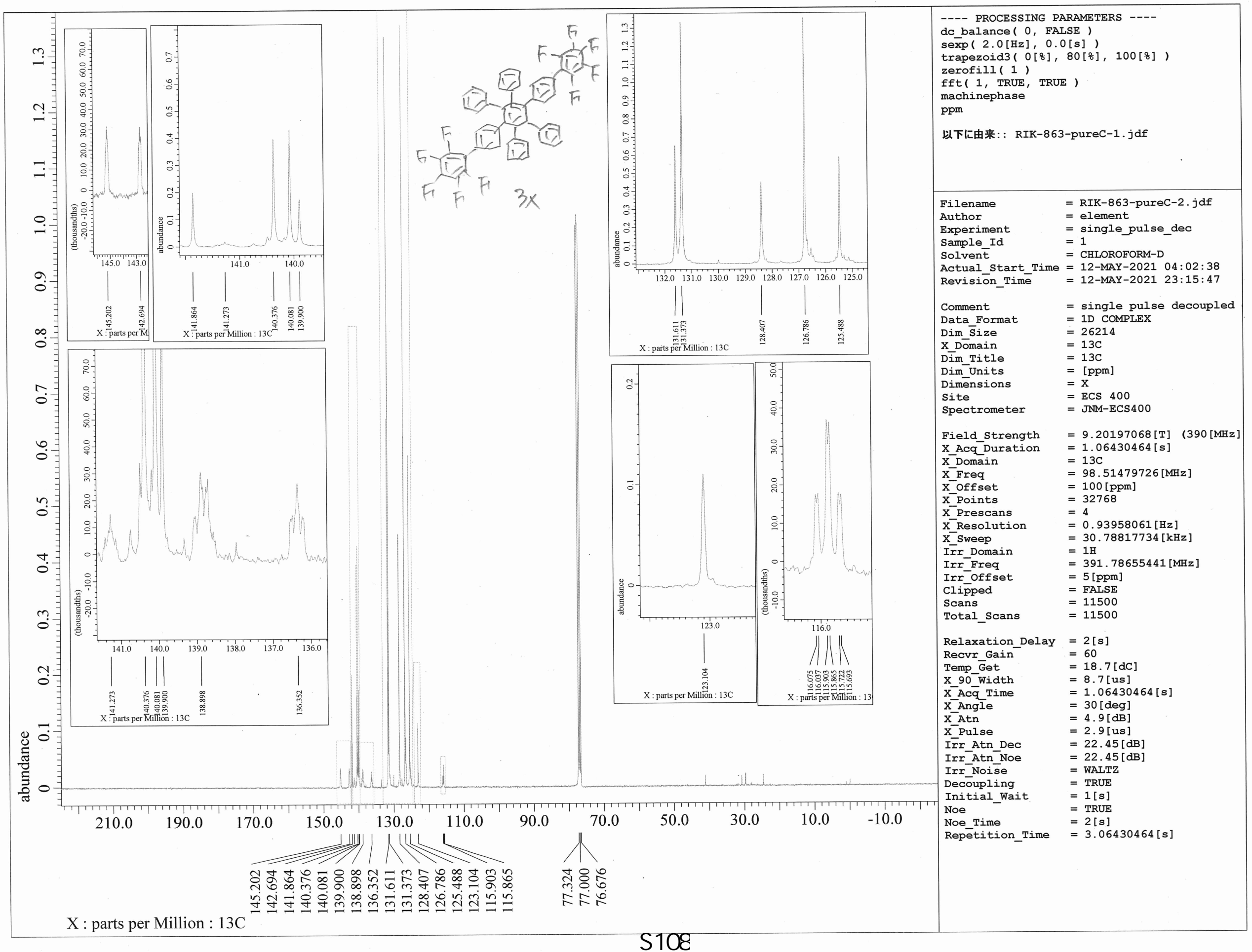




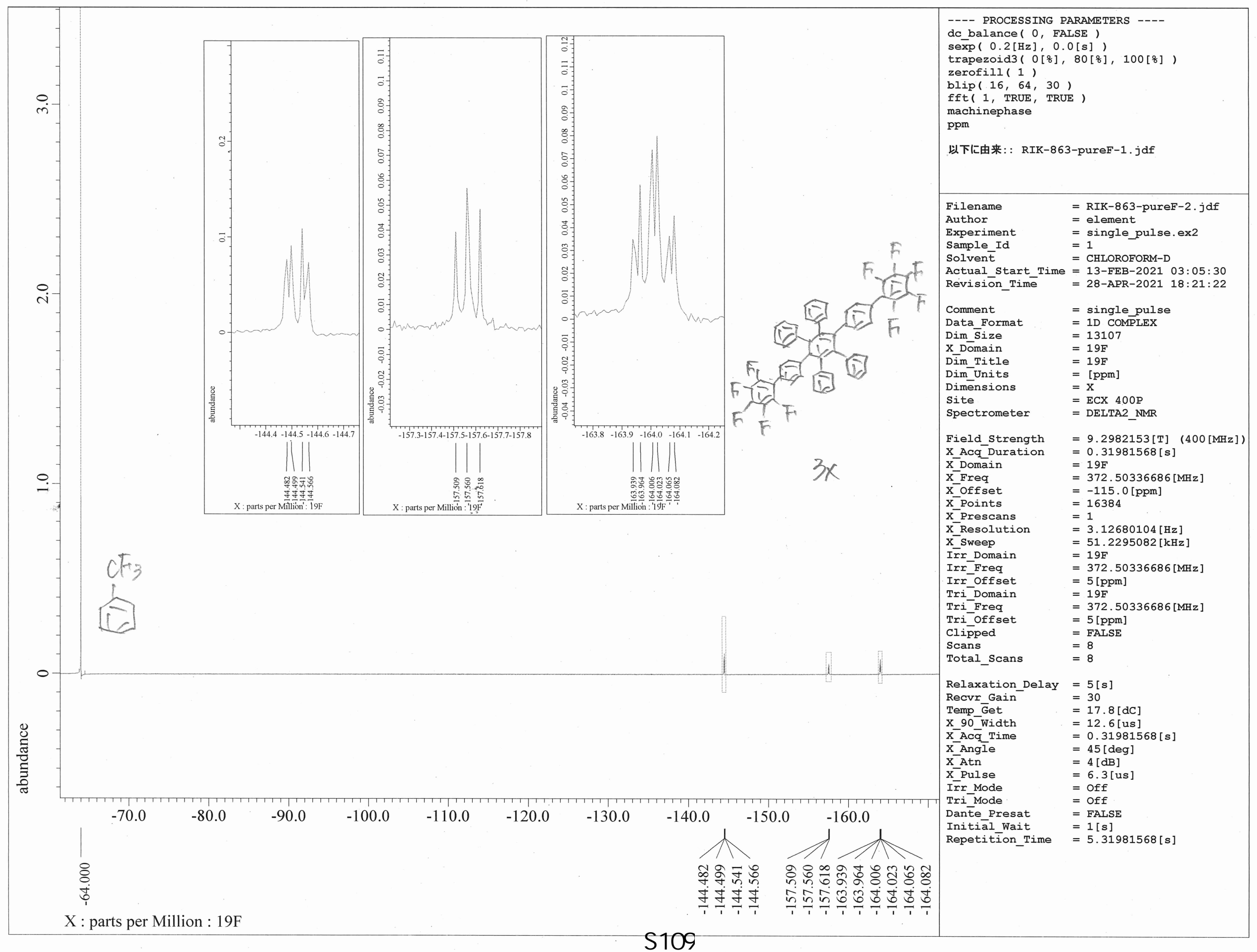




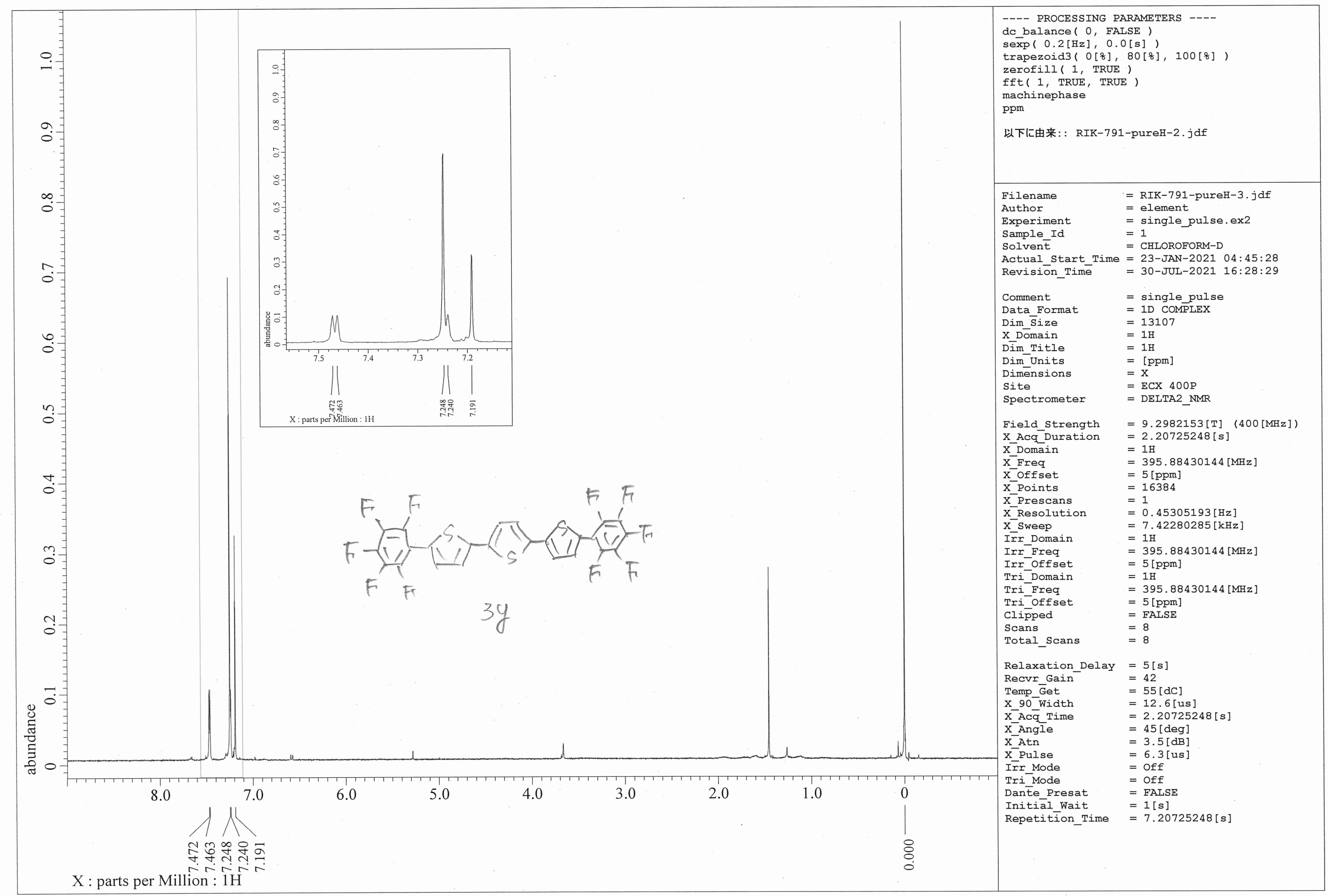




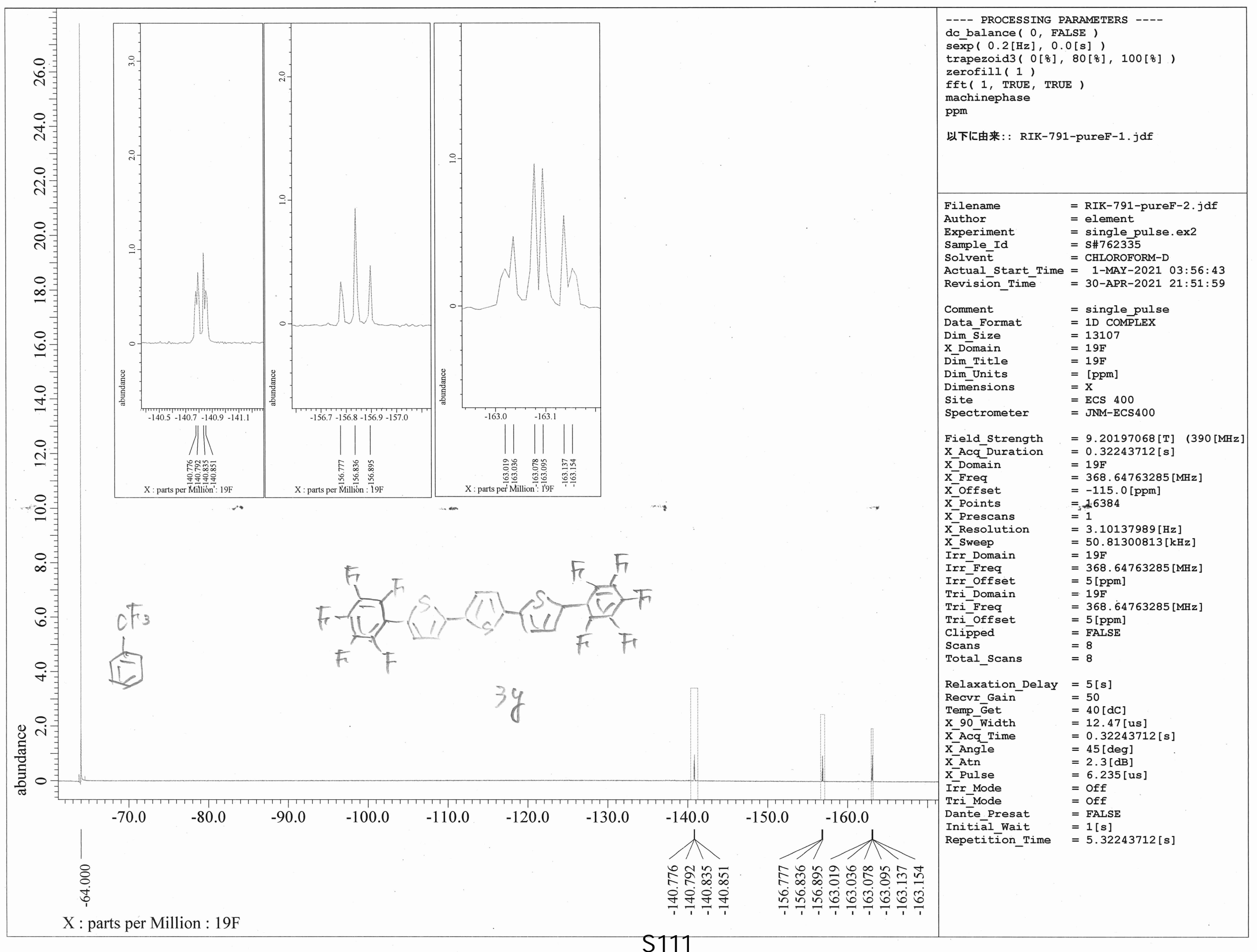




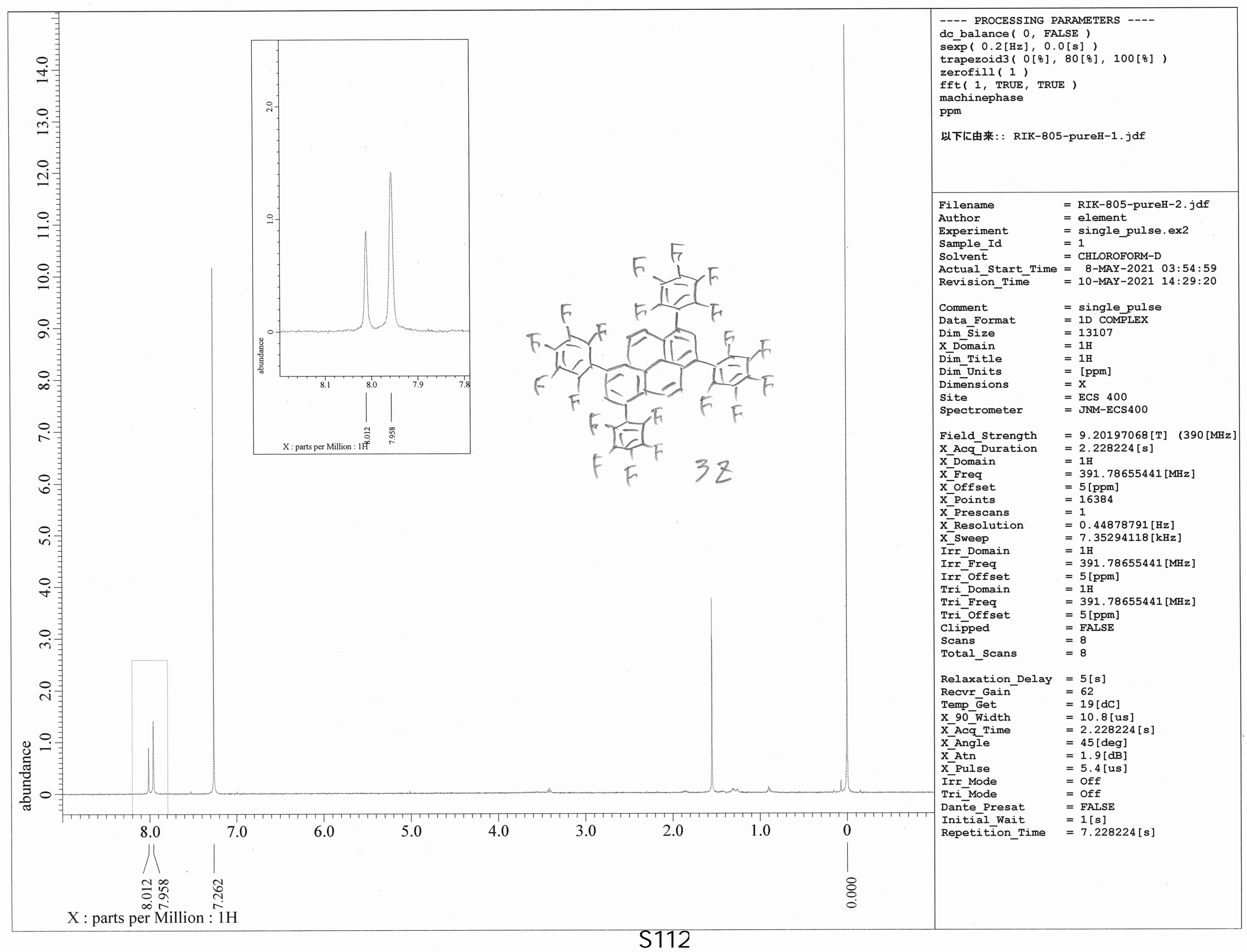




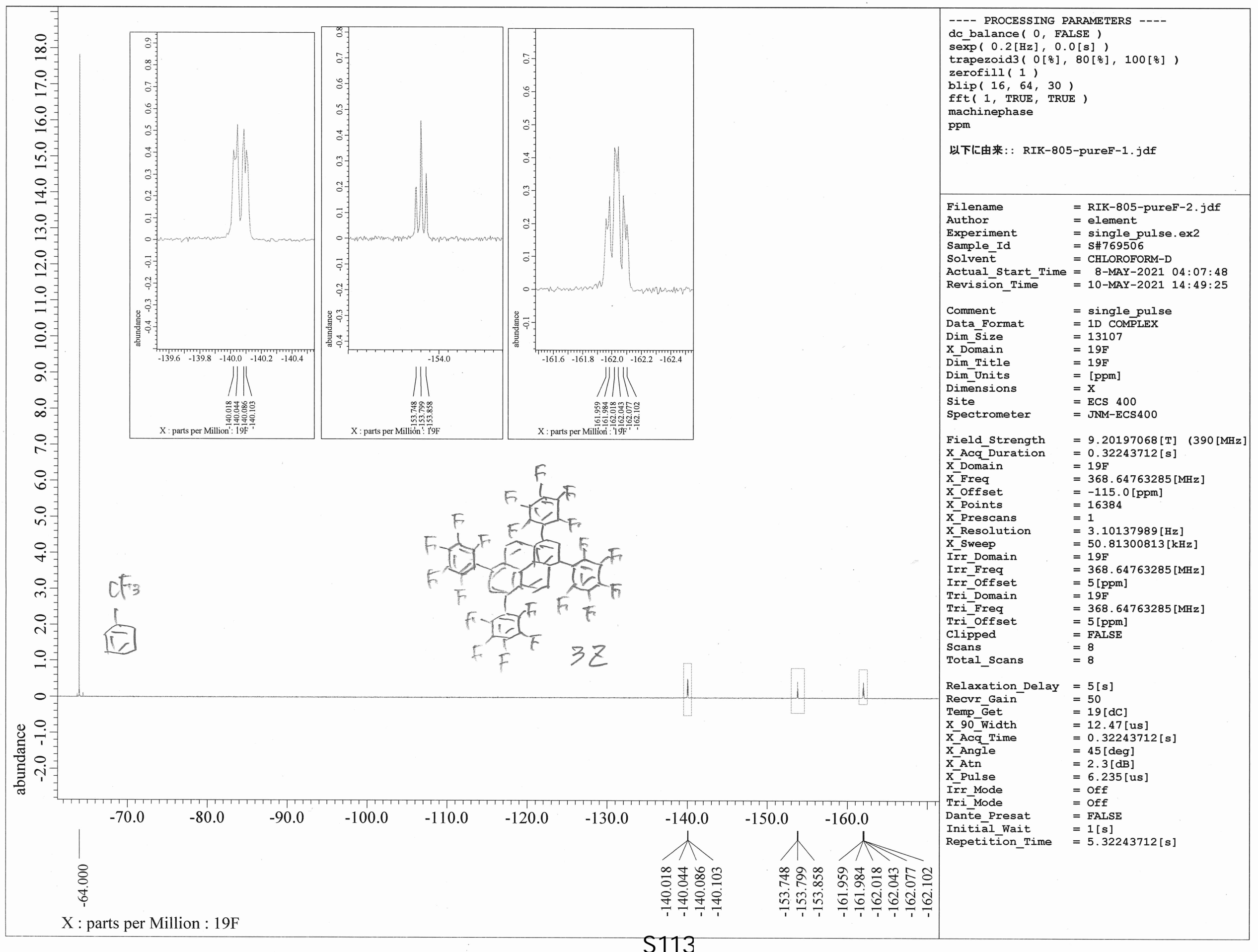




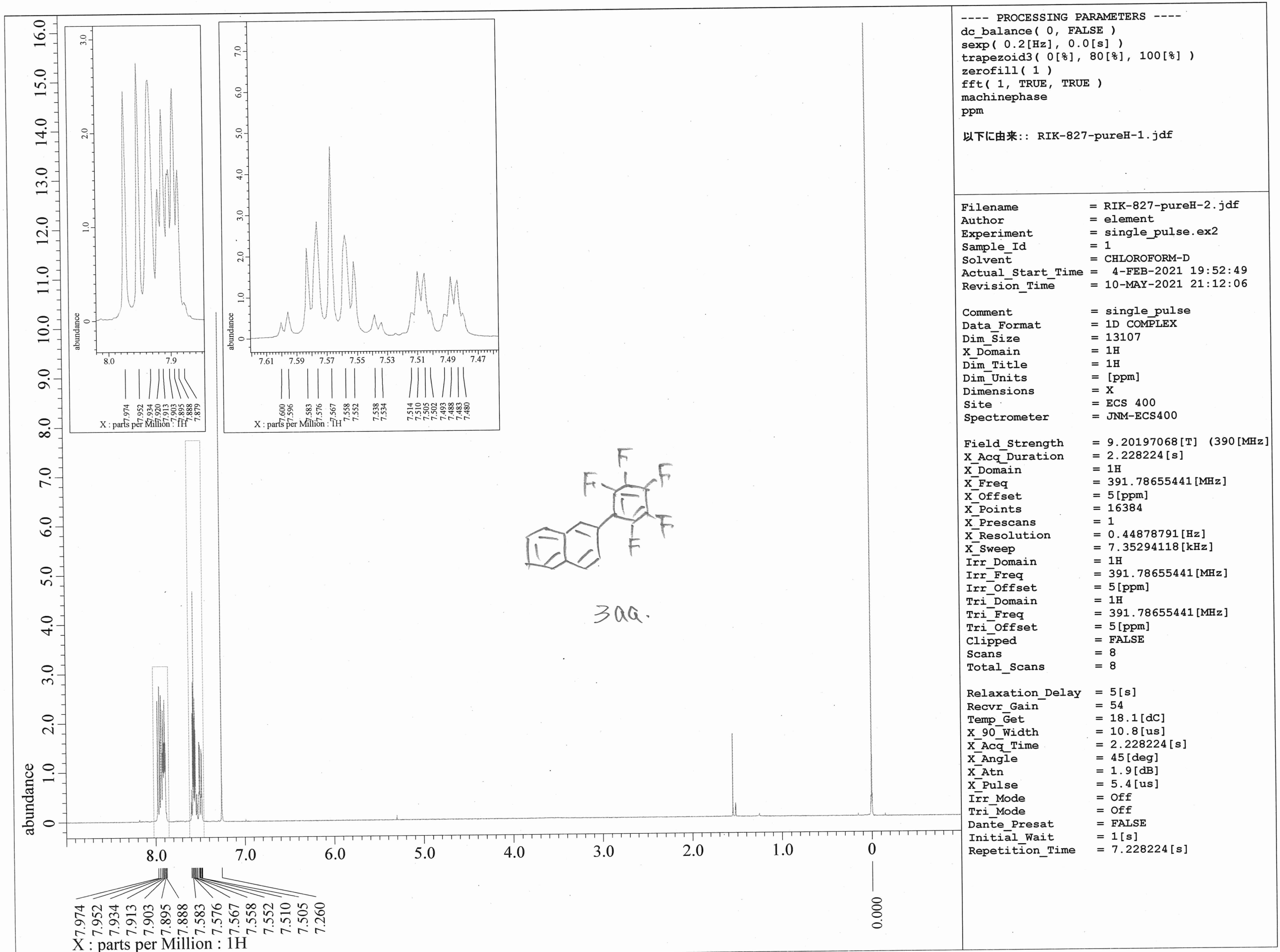

S114 


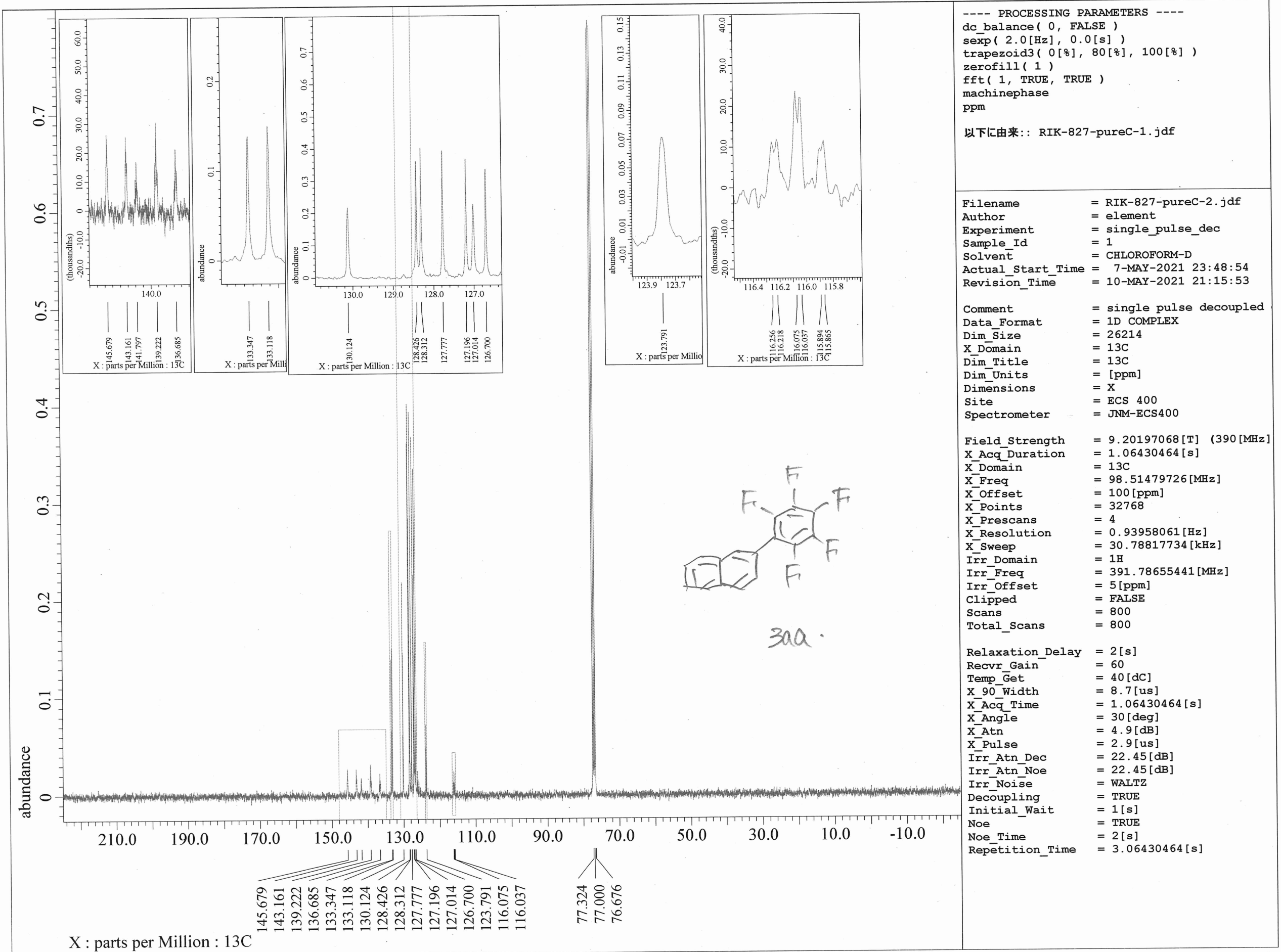




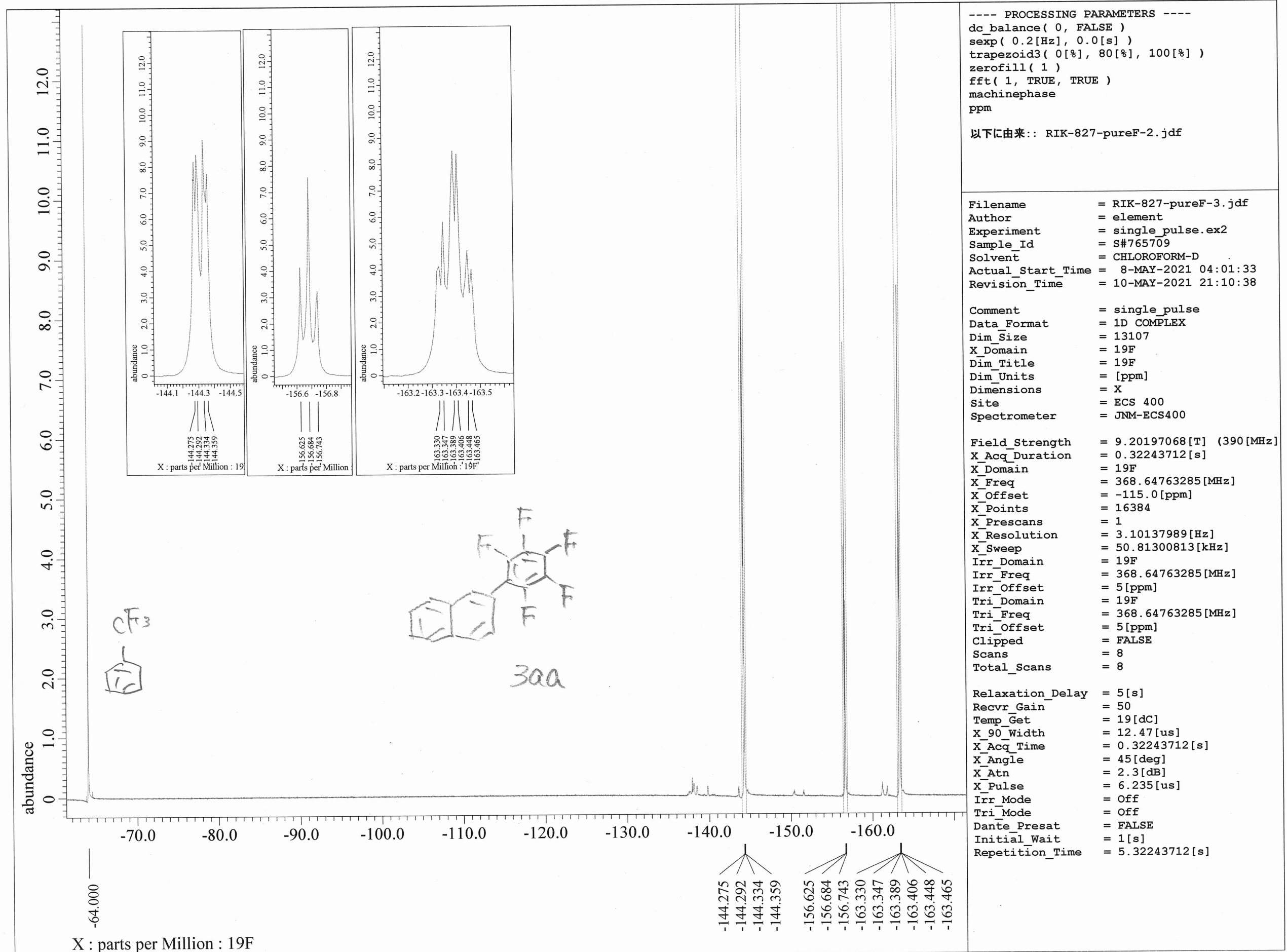




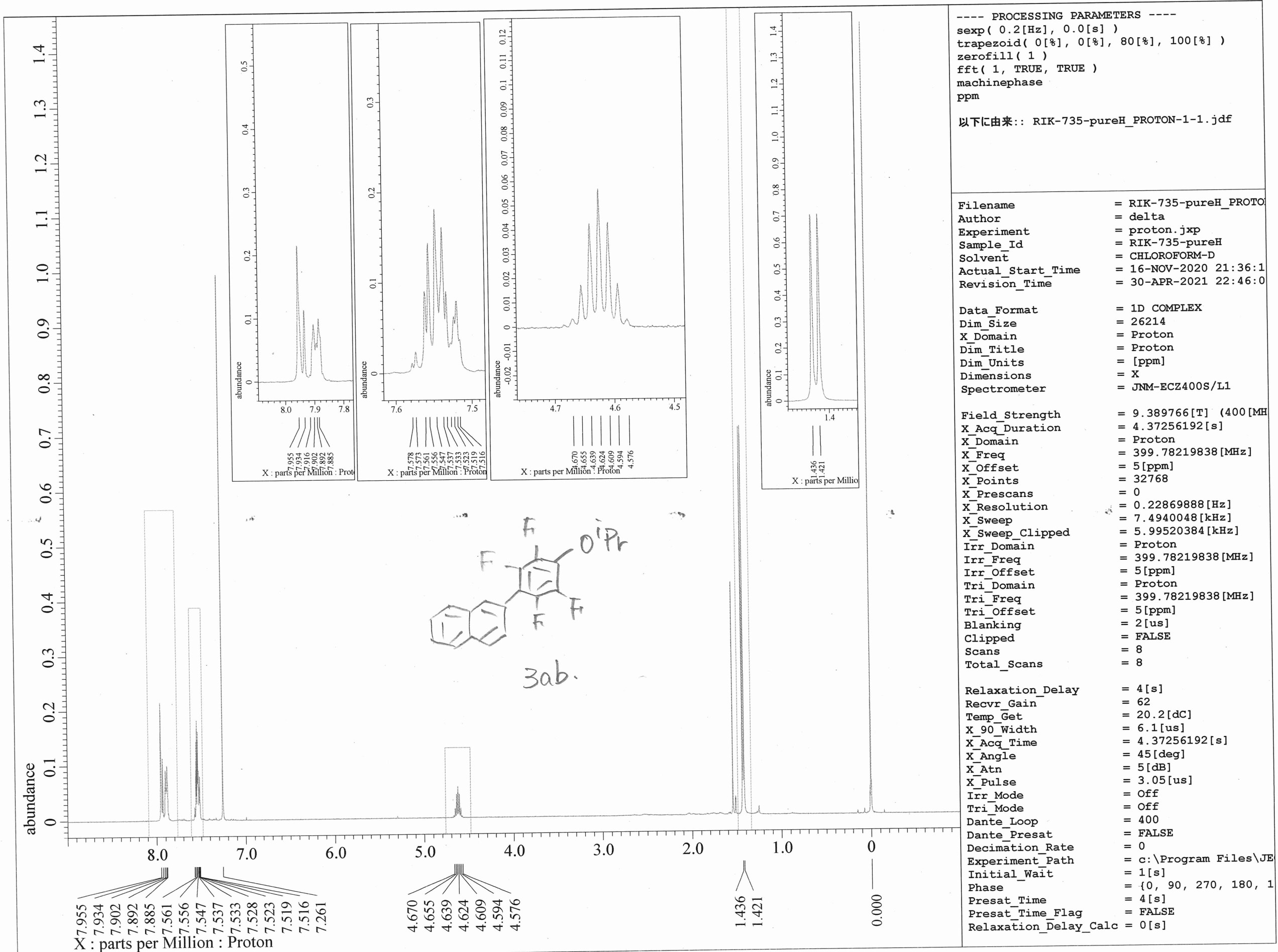




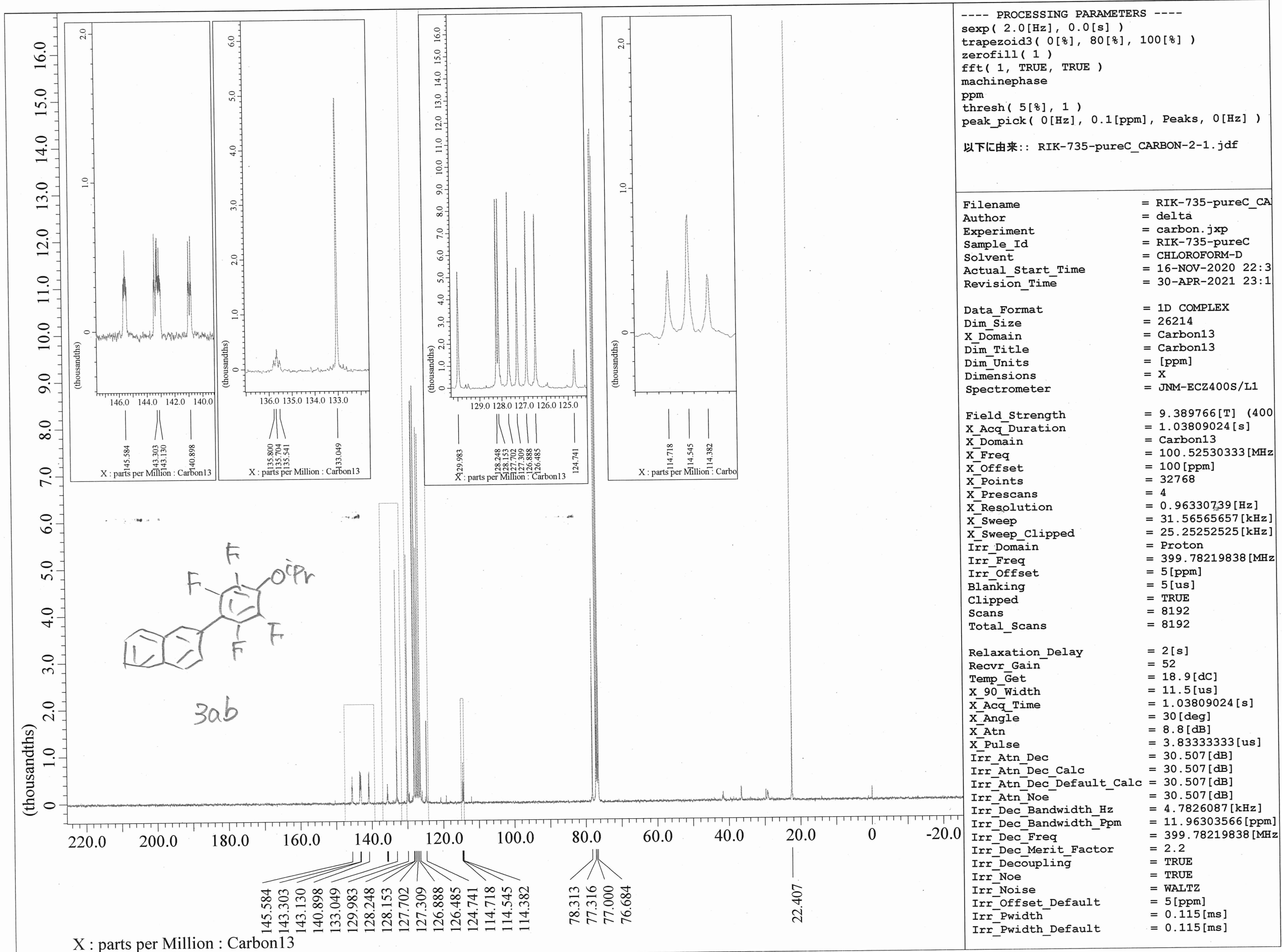




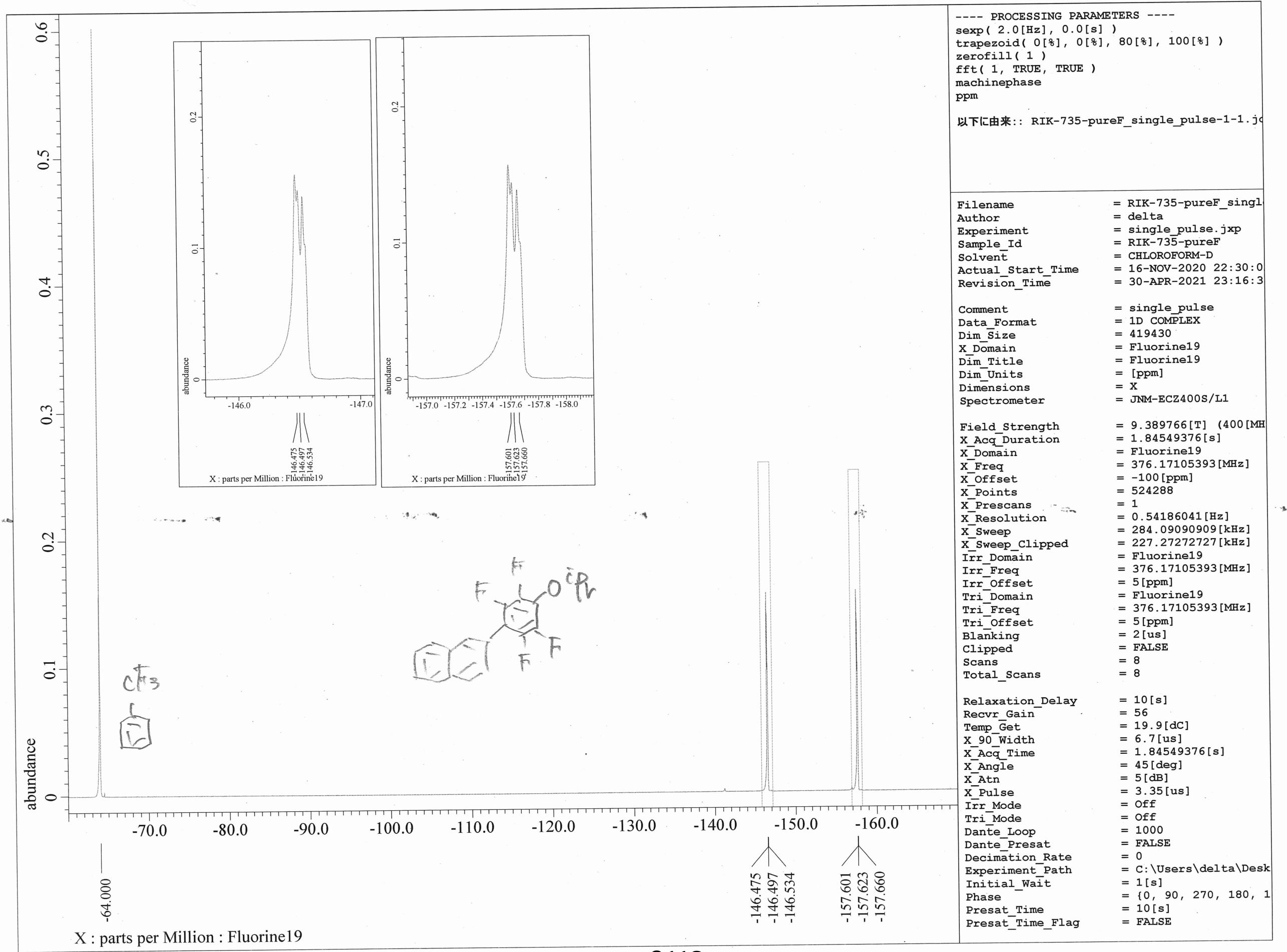




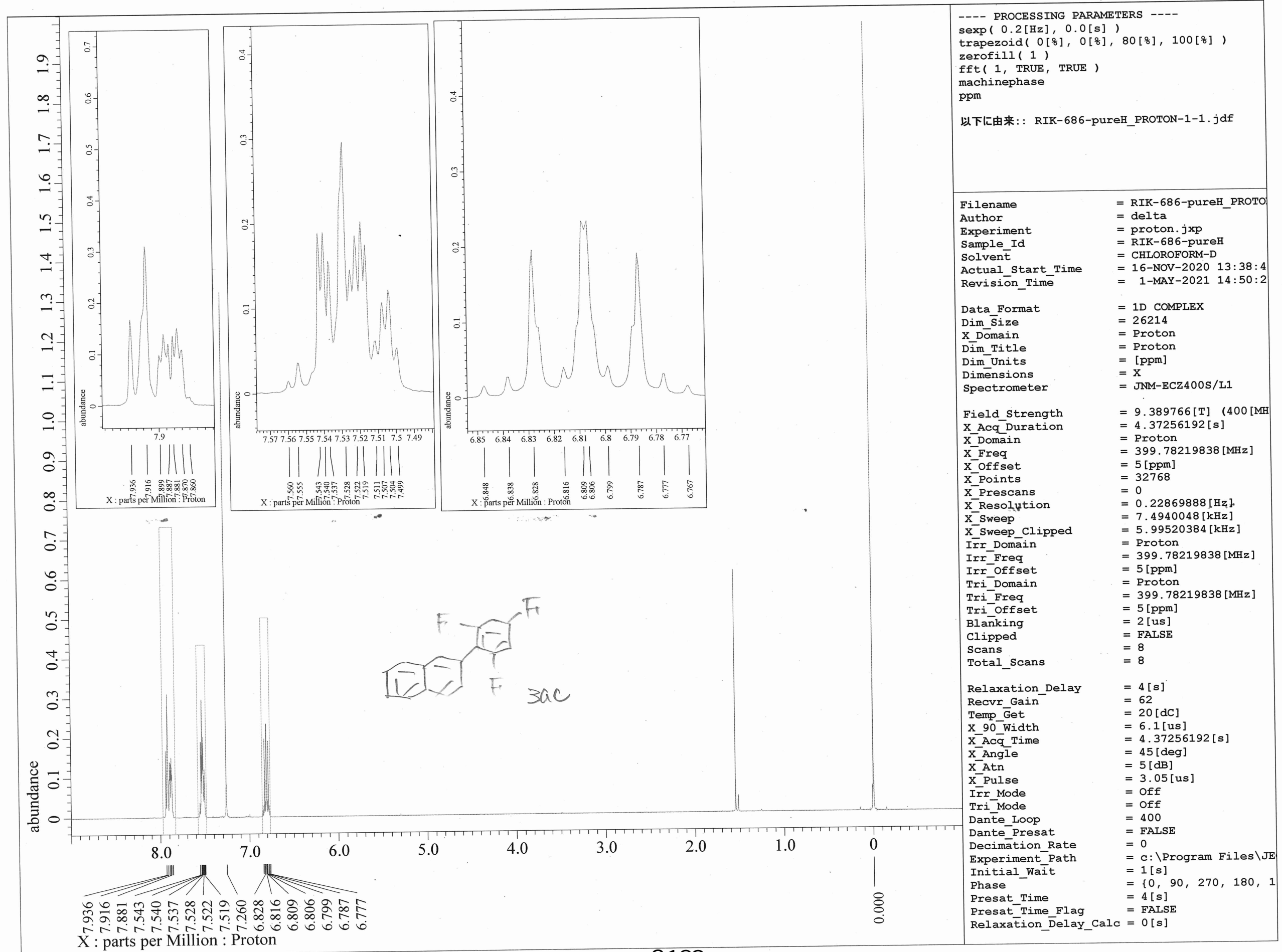




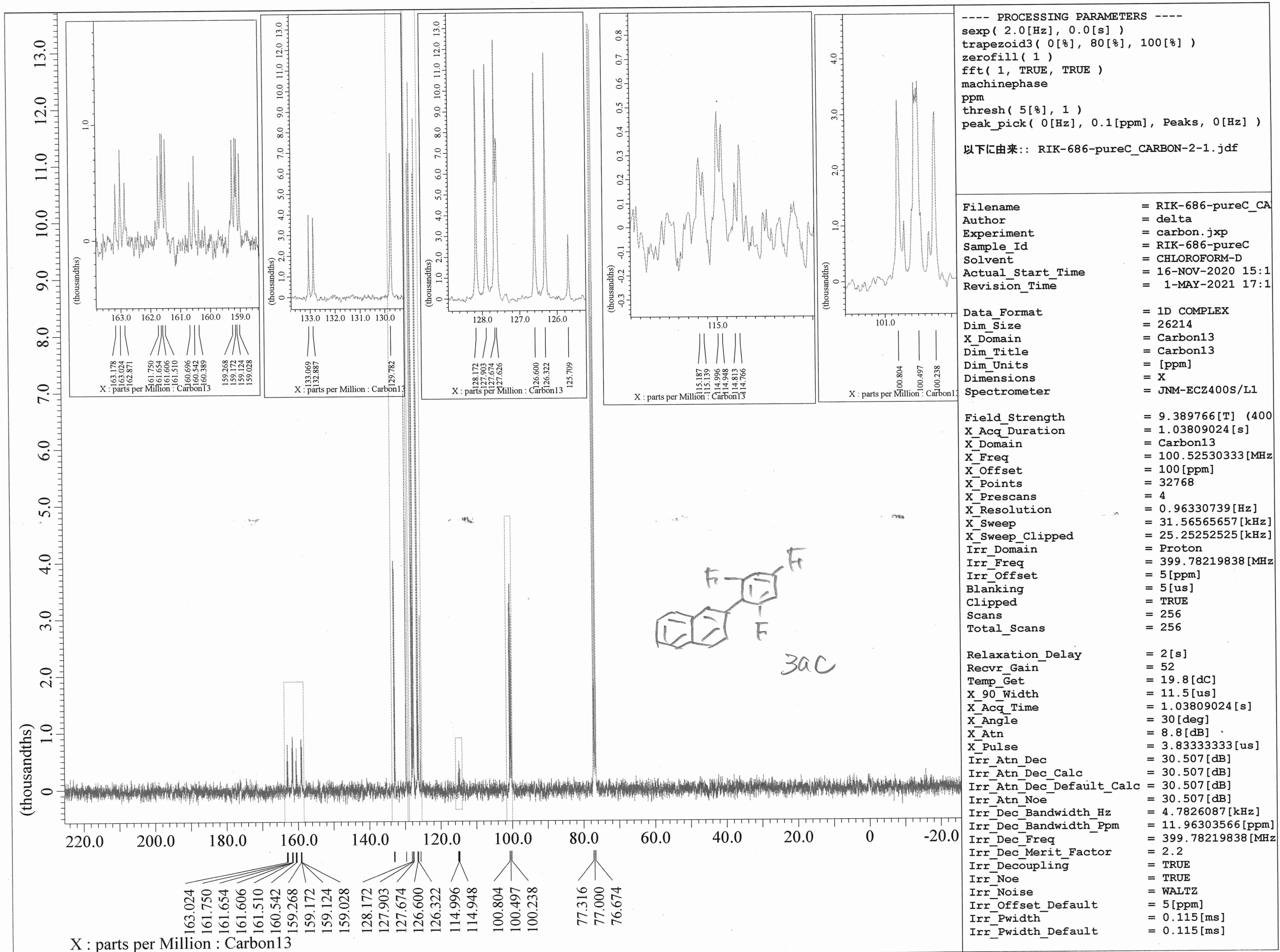




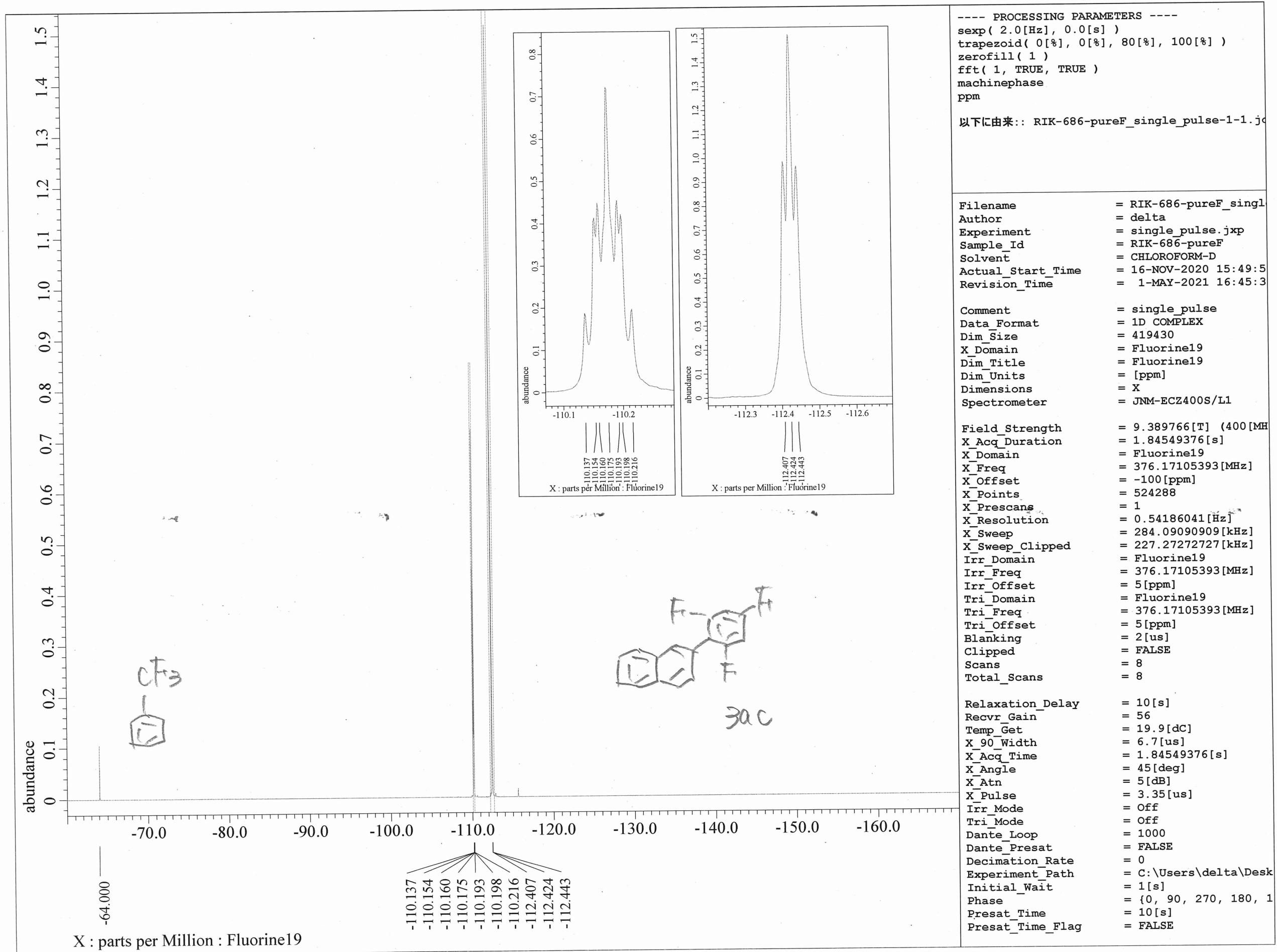




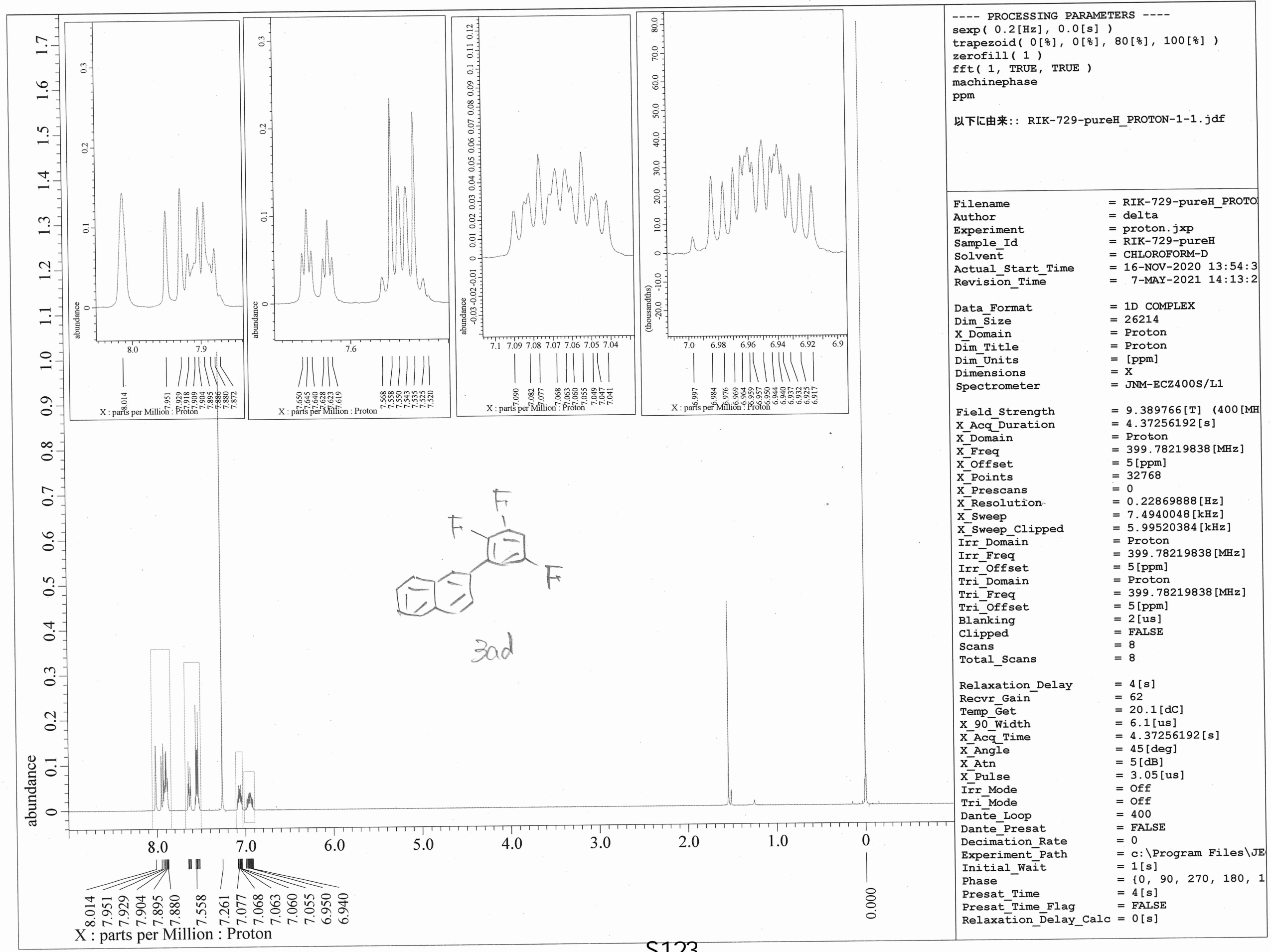




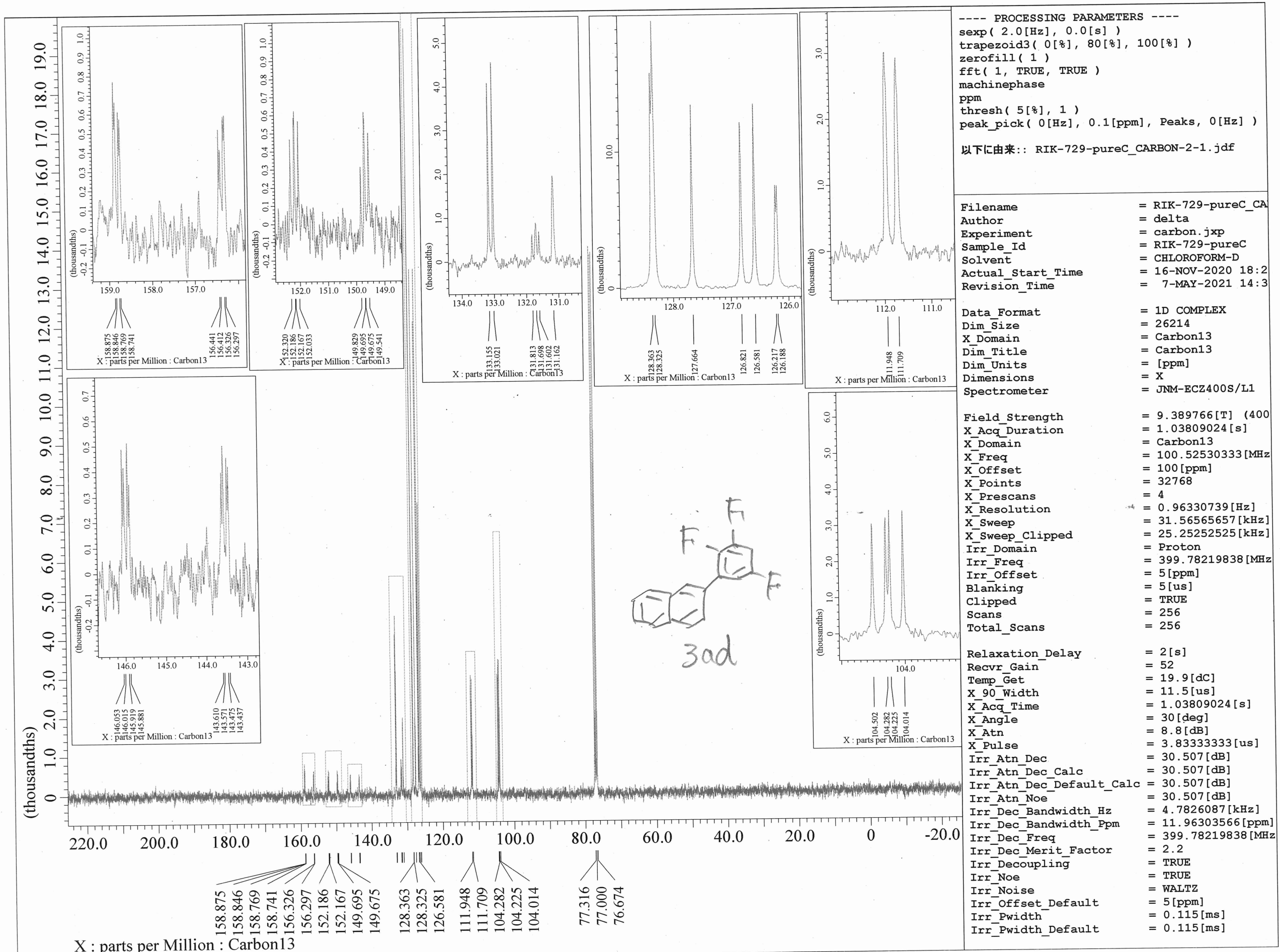




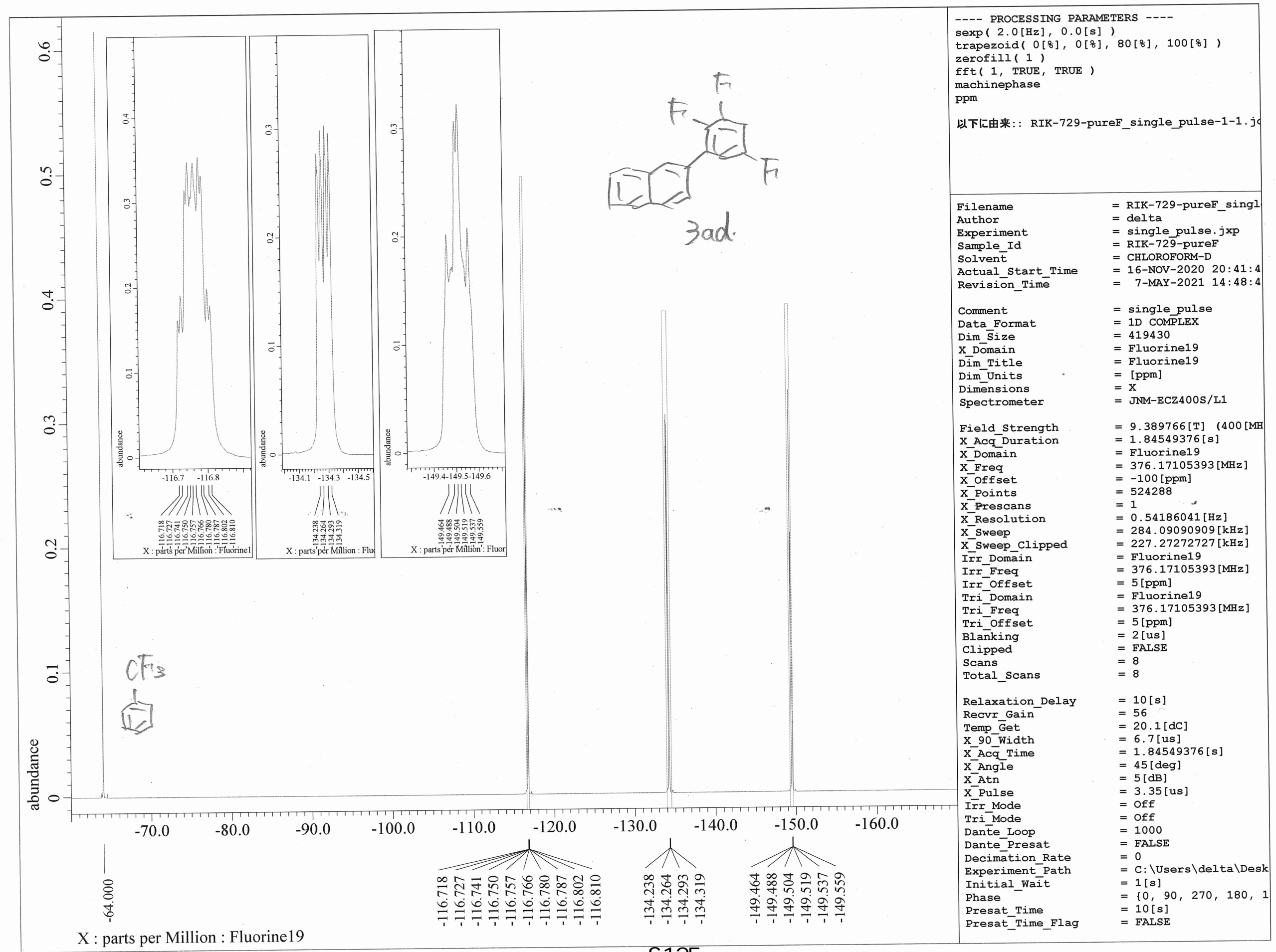

S125 


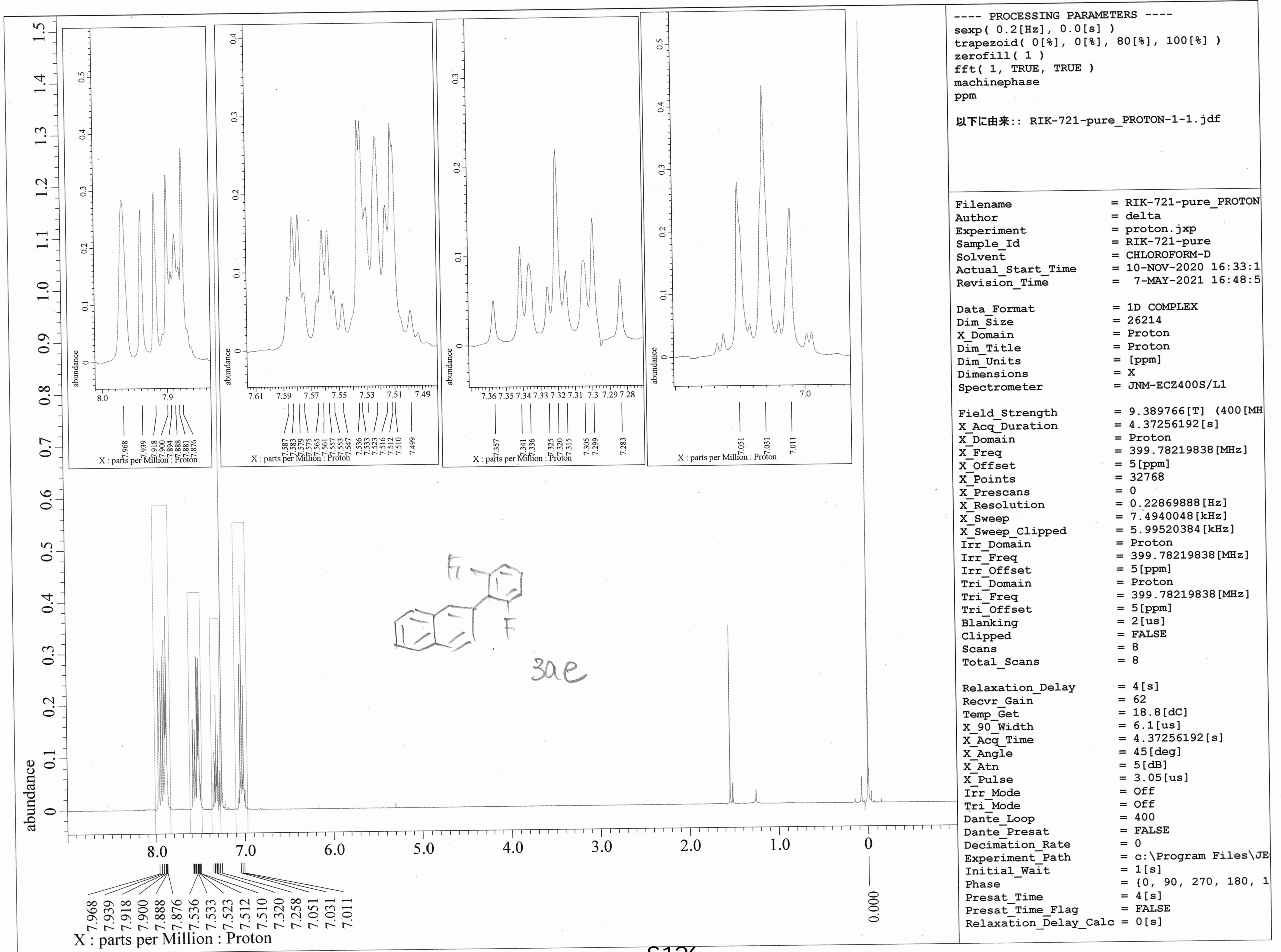




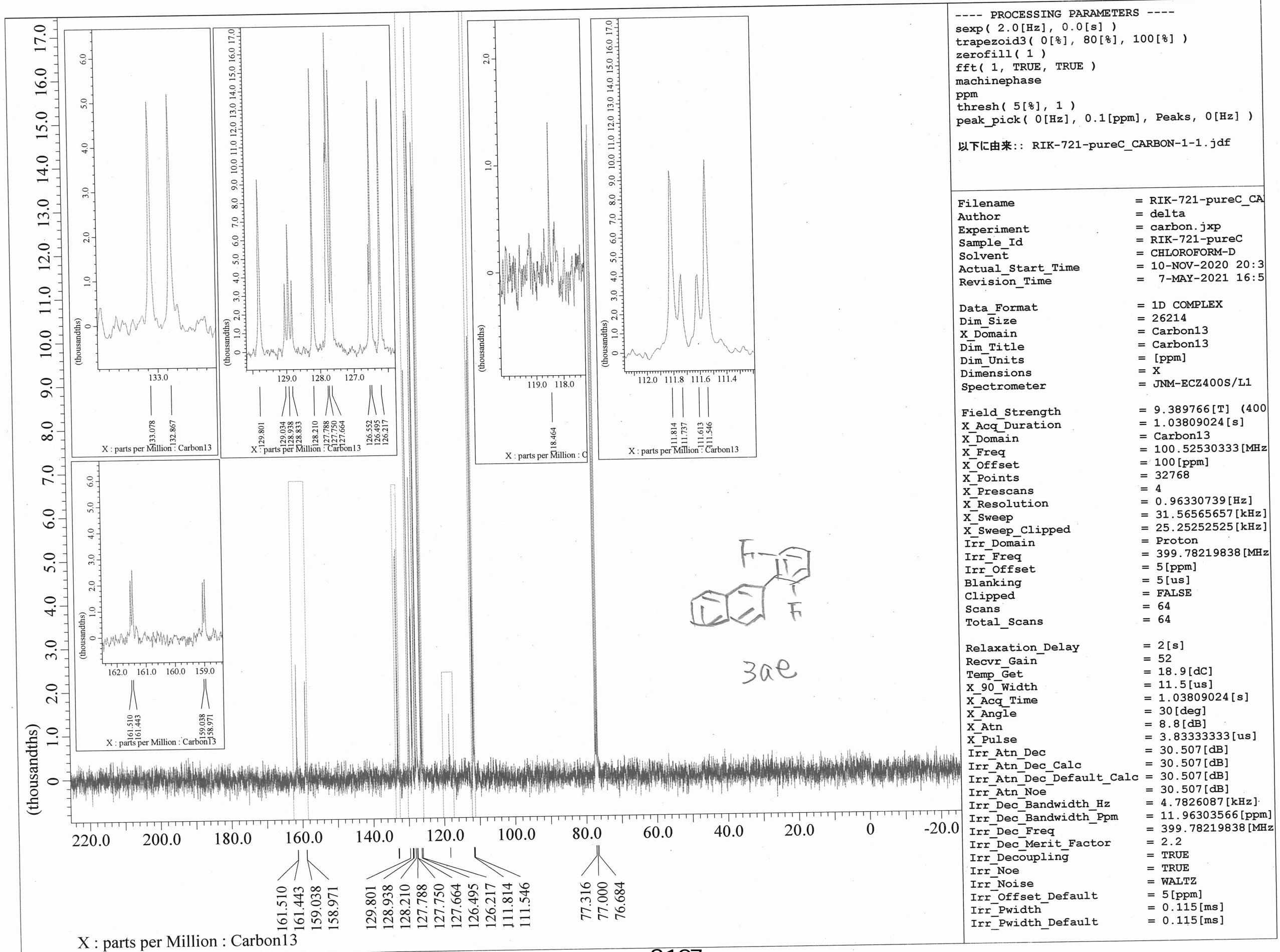




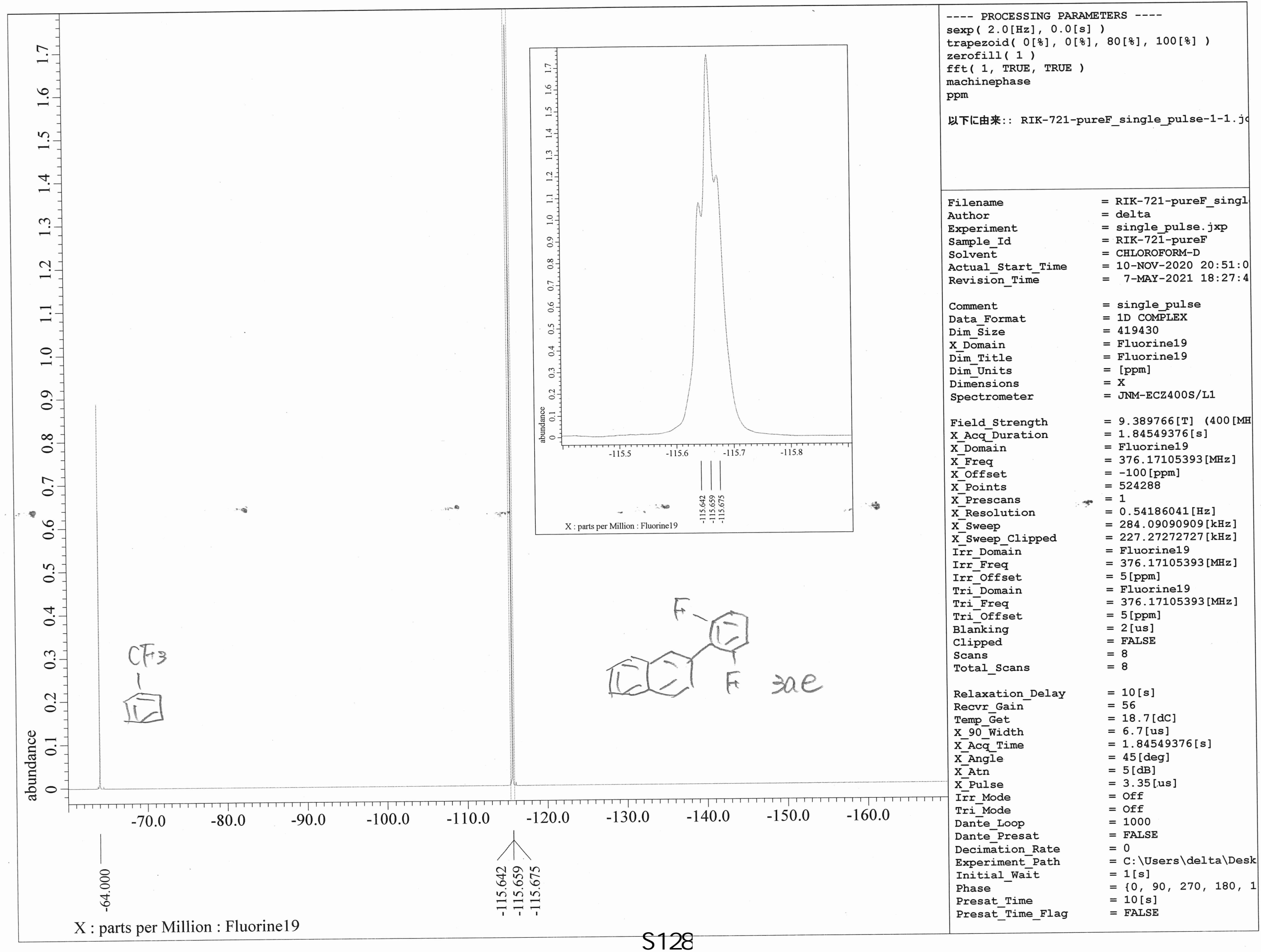




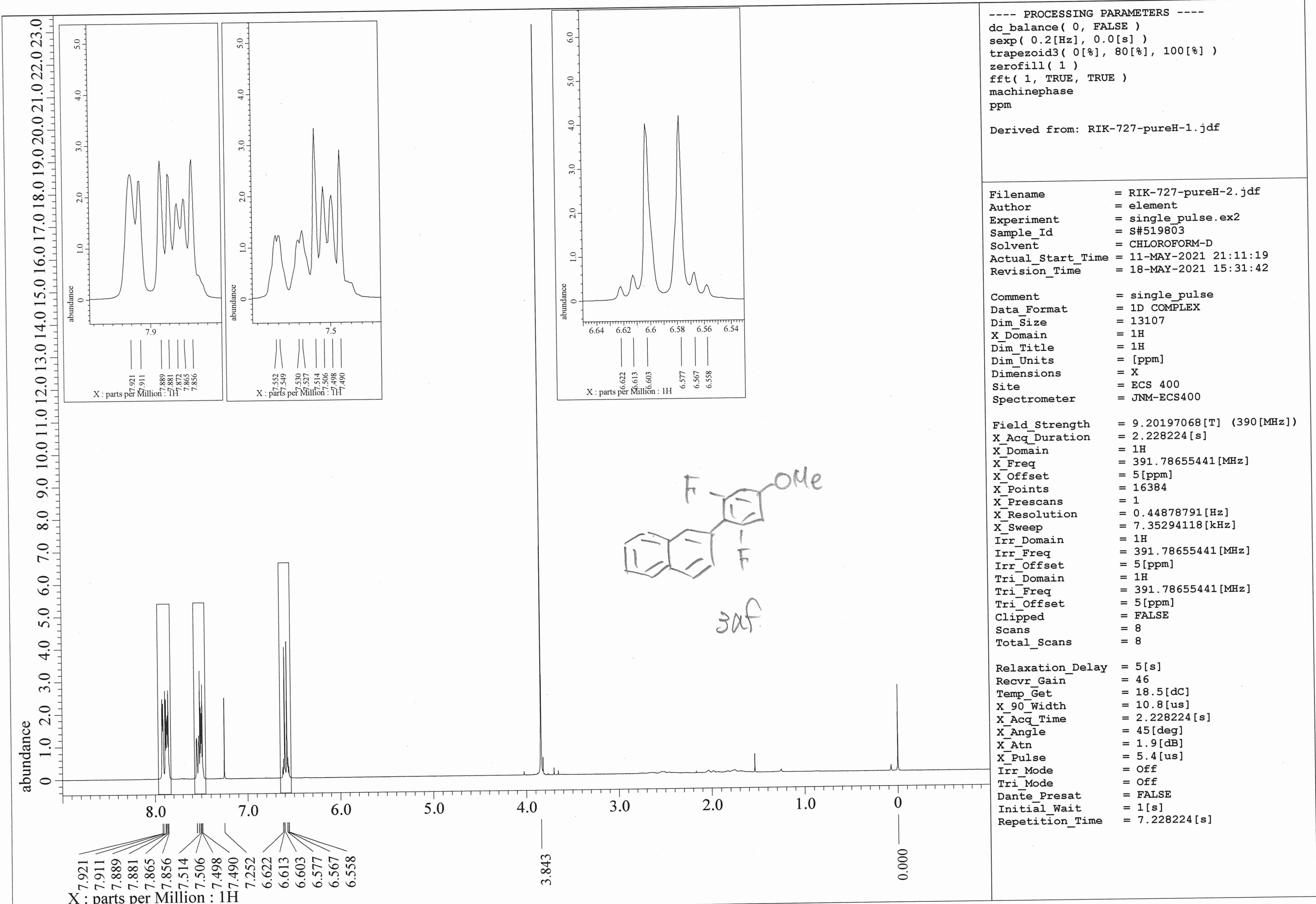




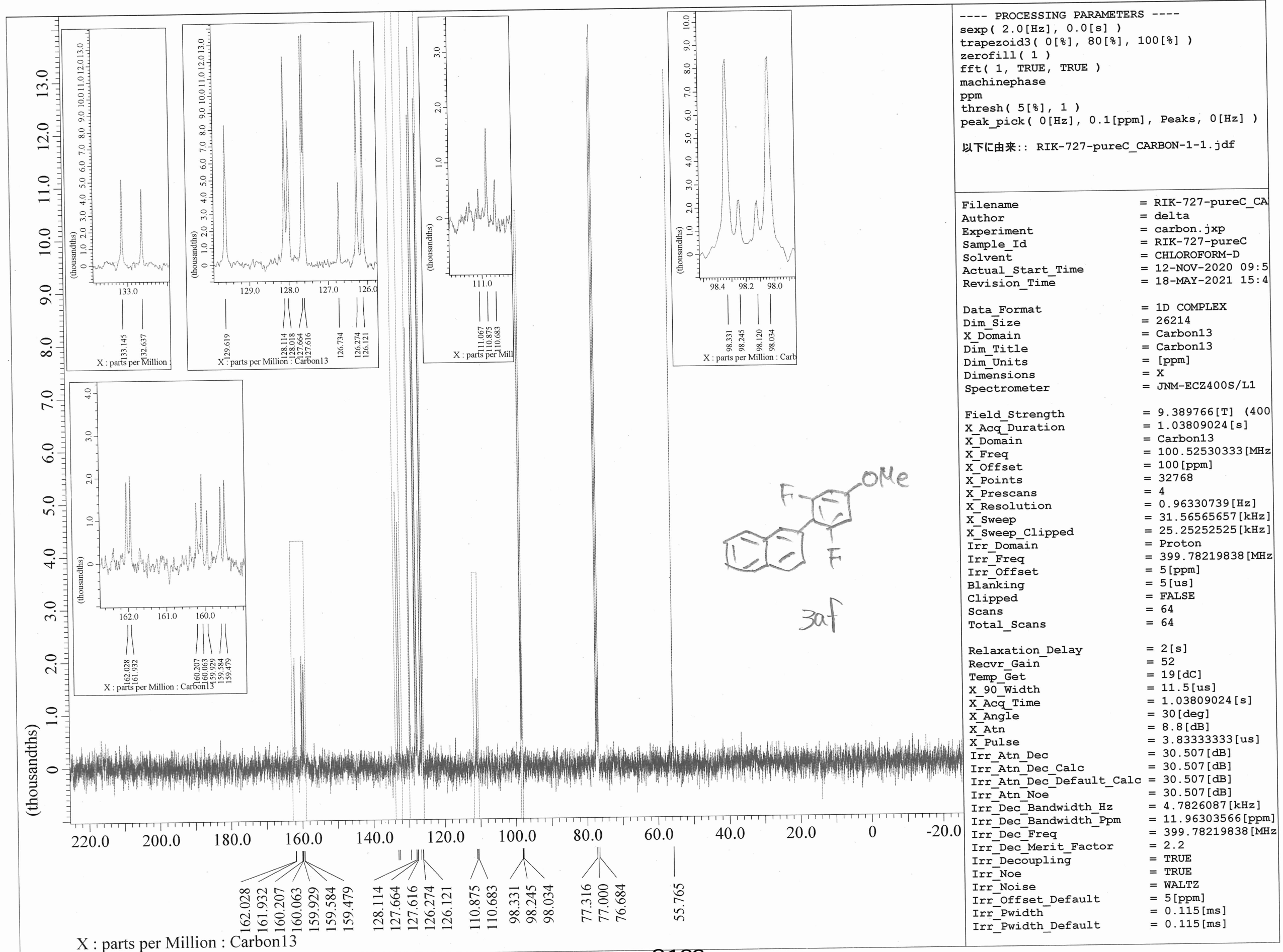




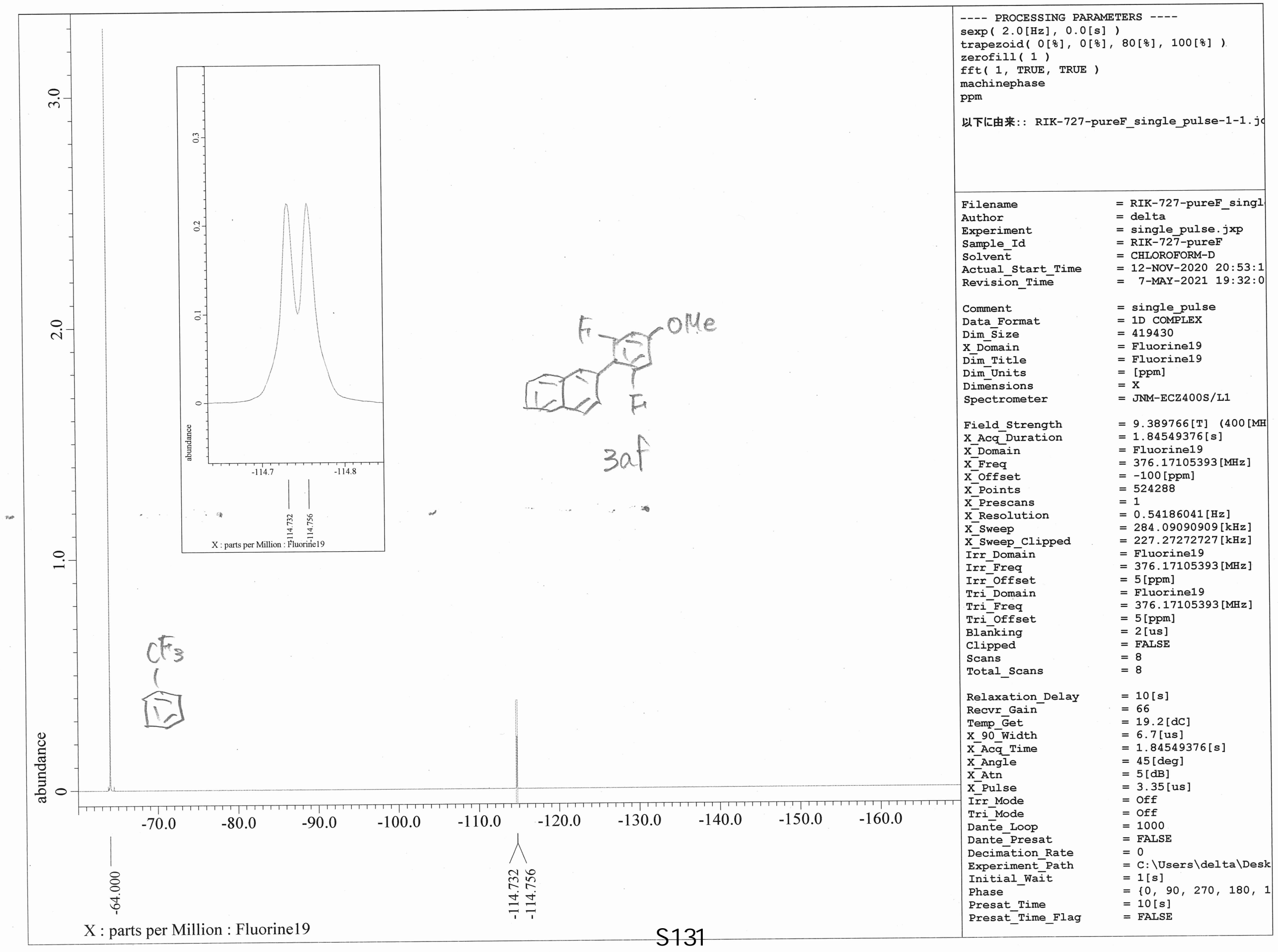




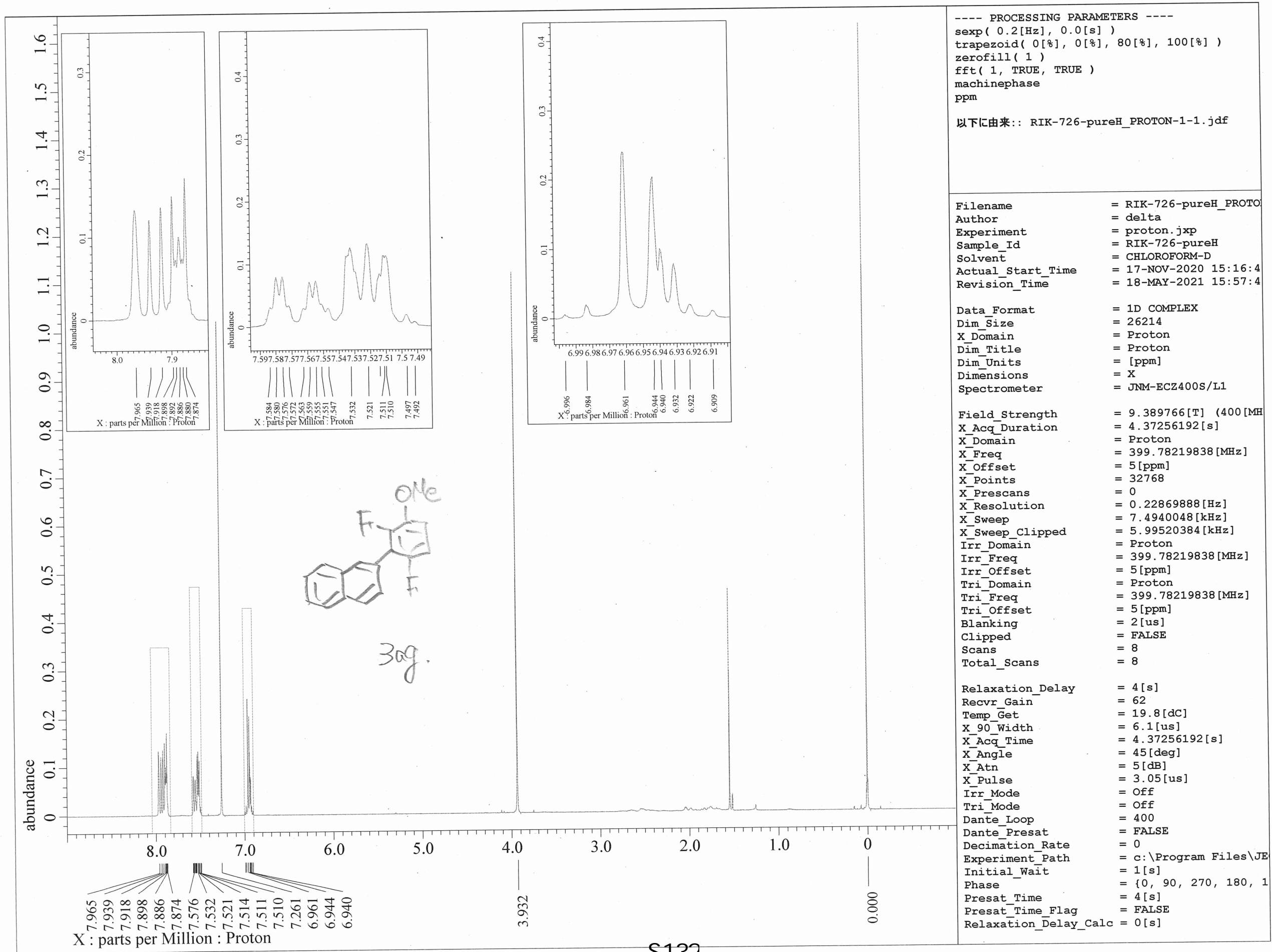




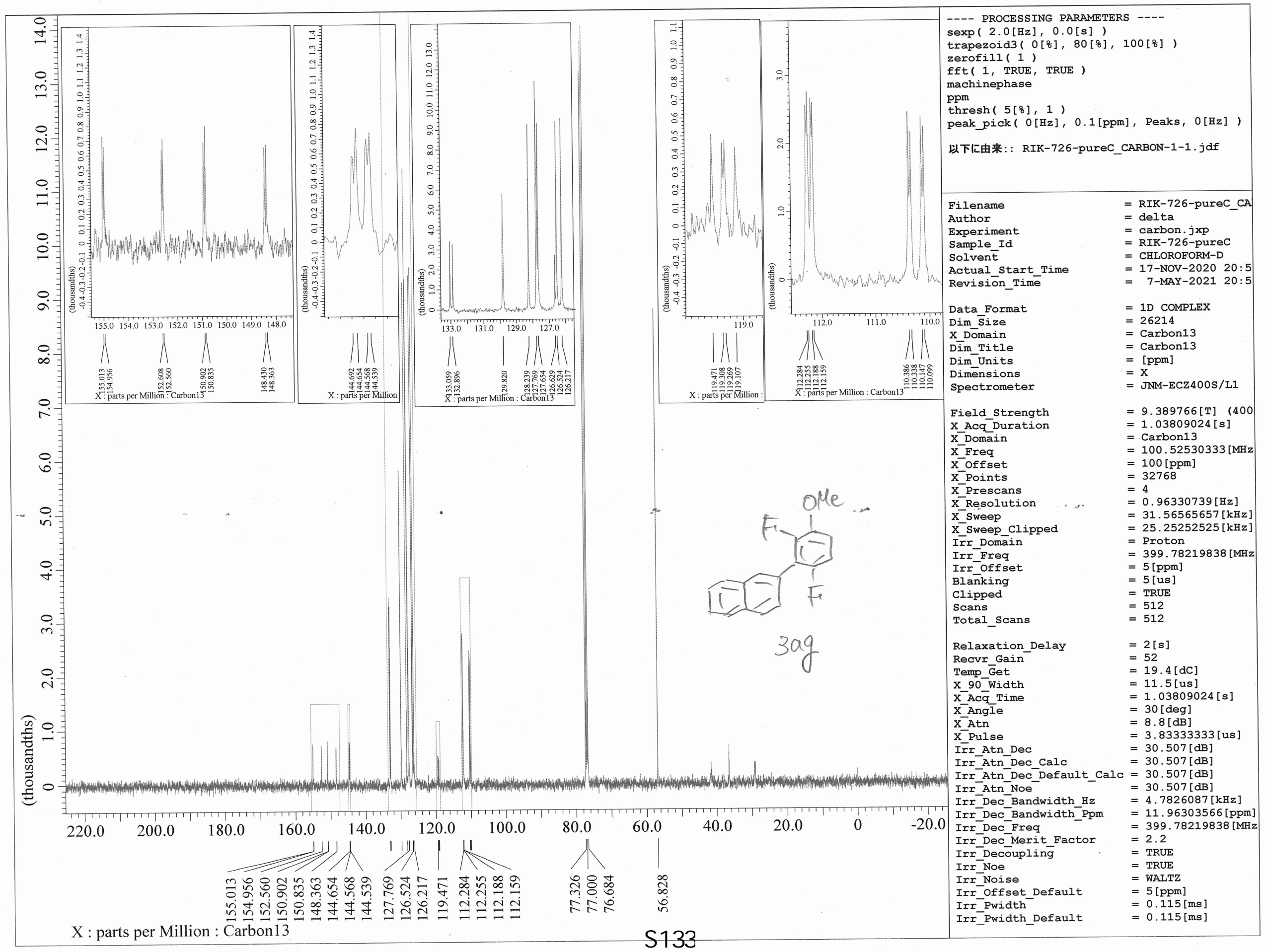




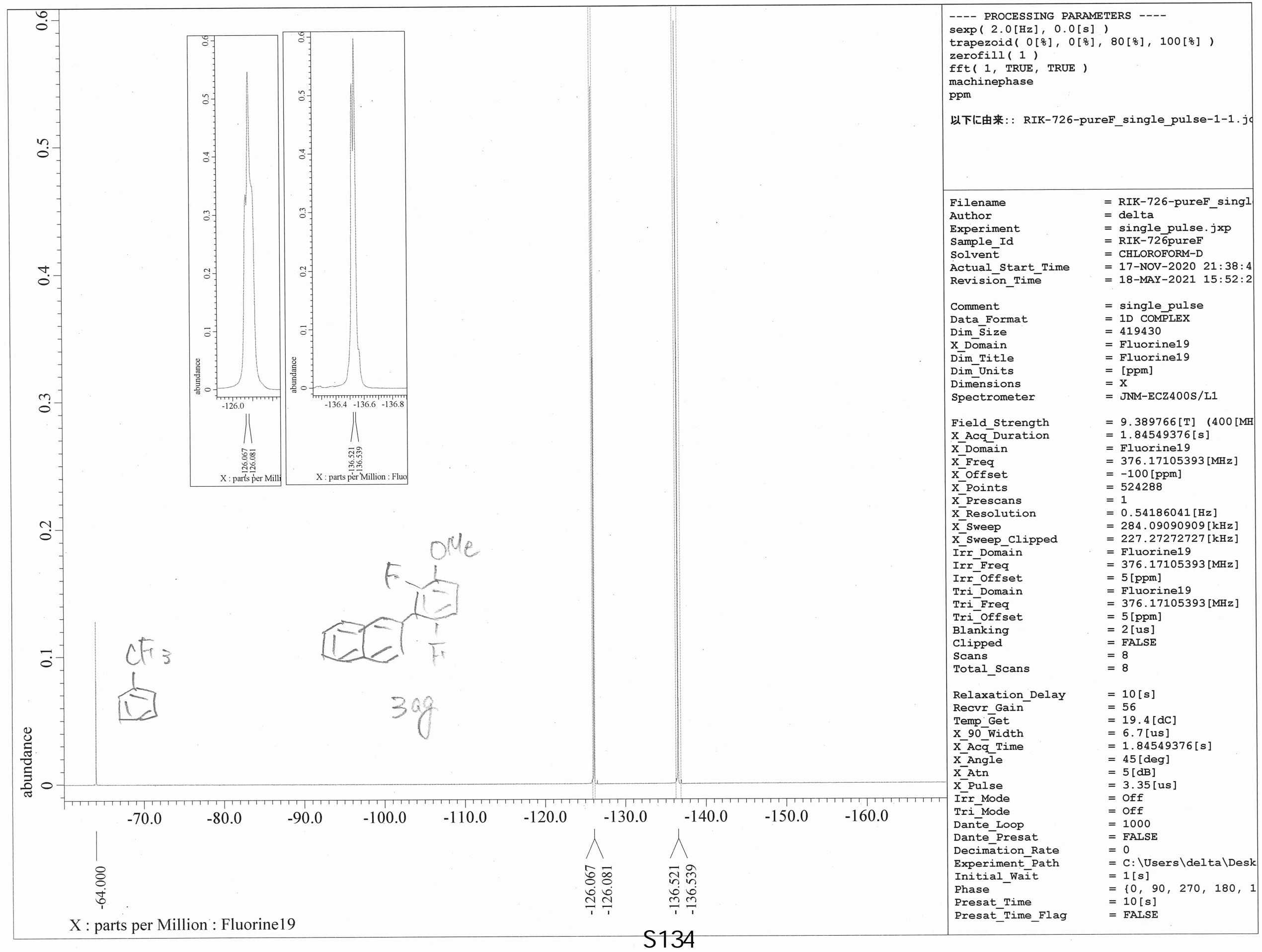




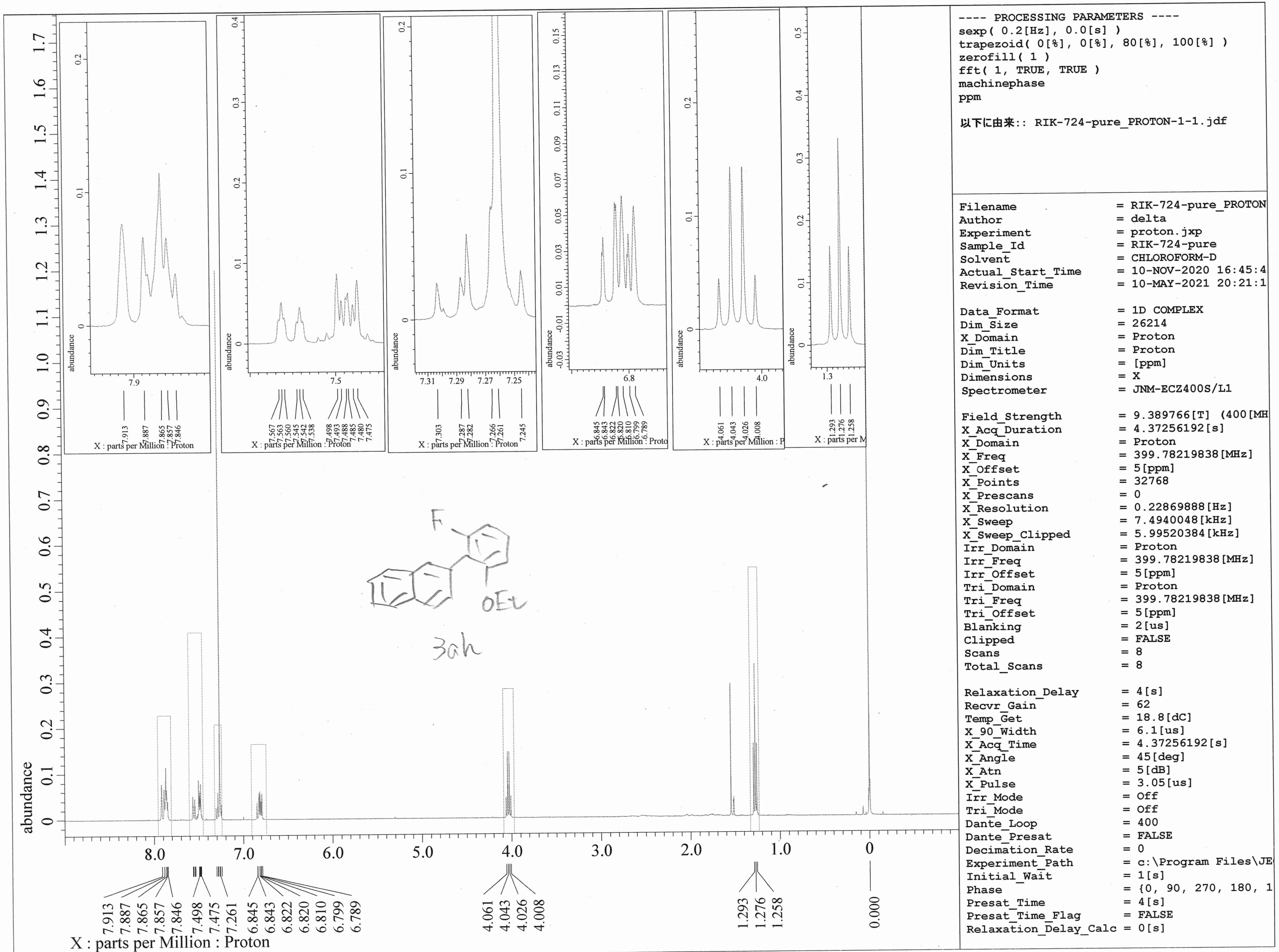




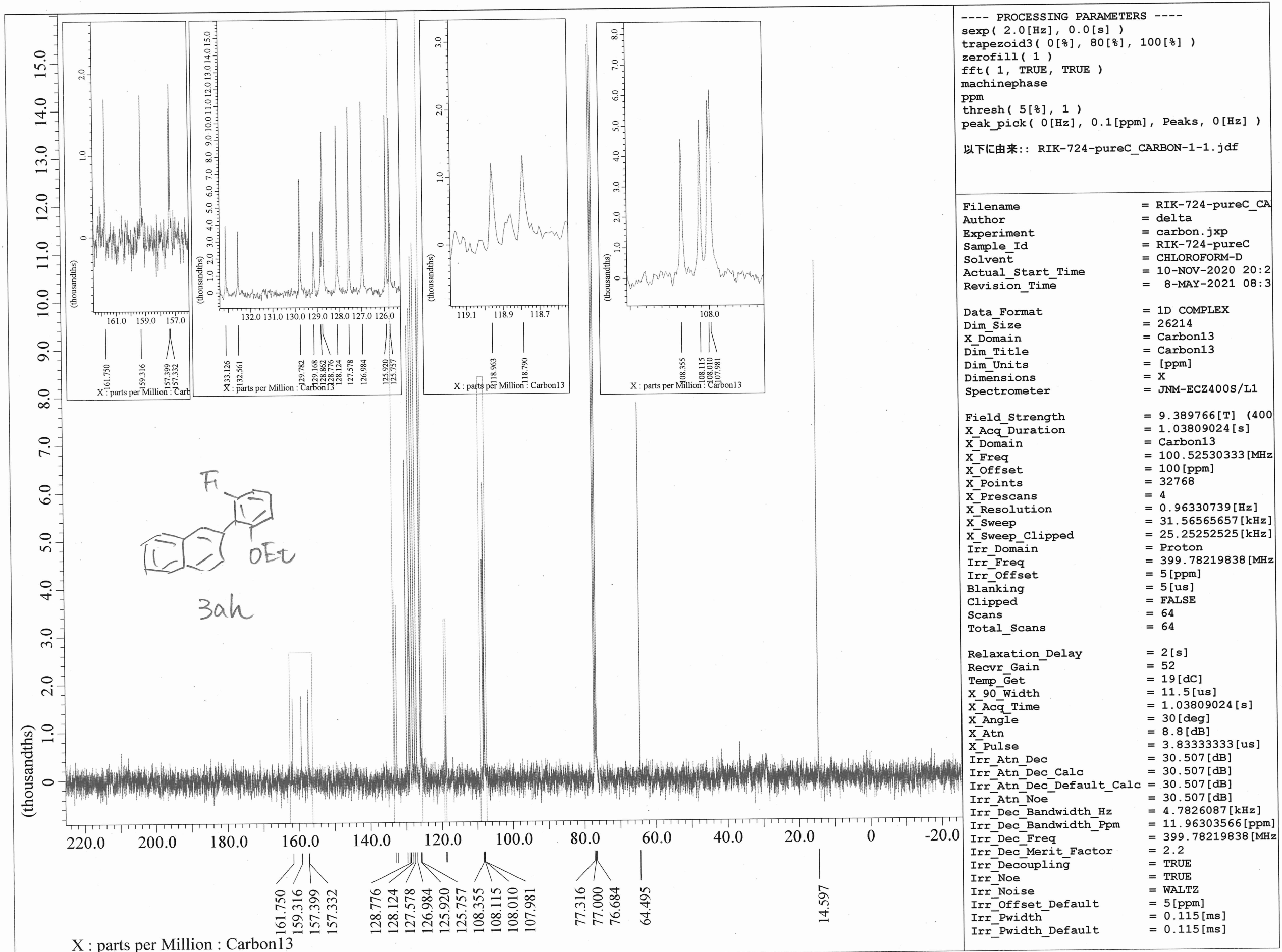

S136 


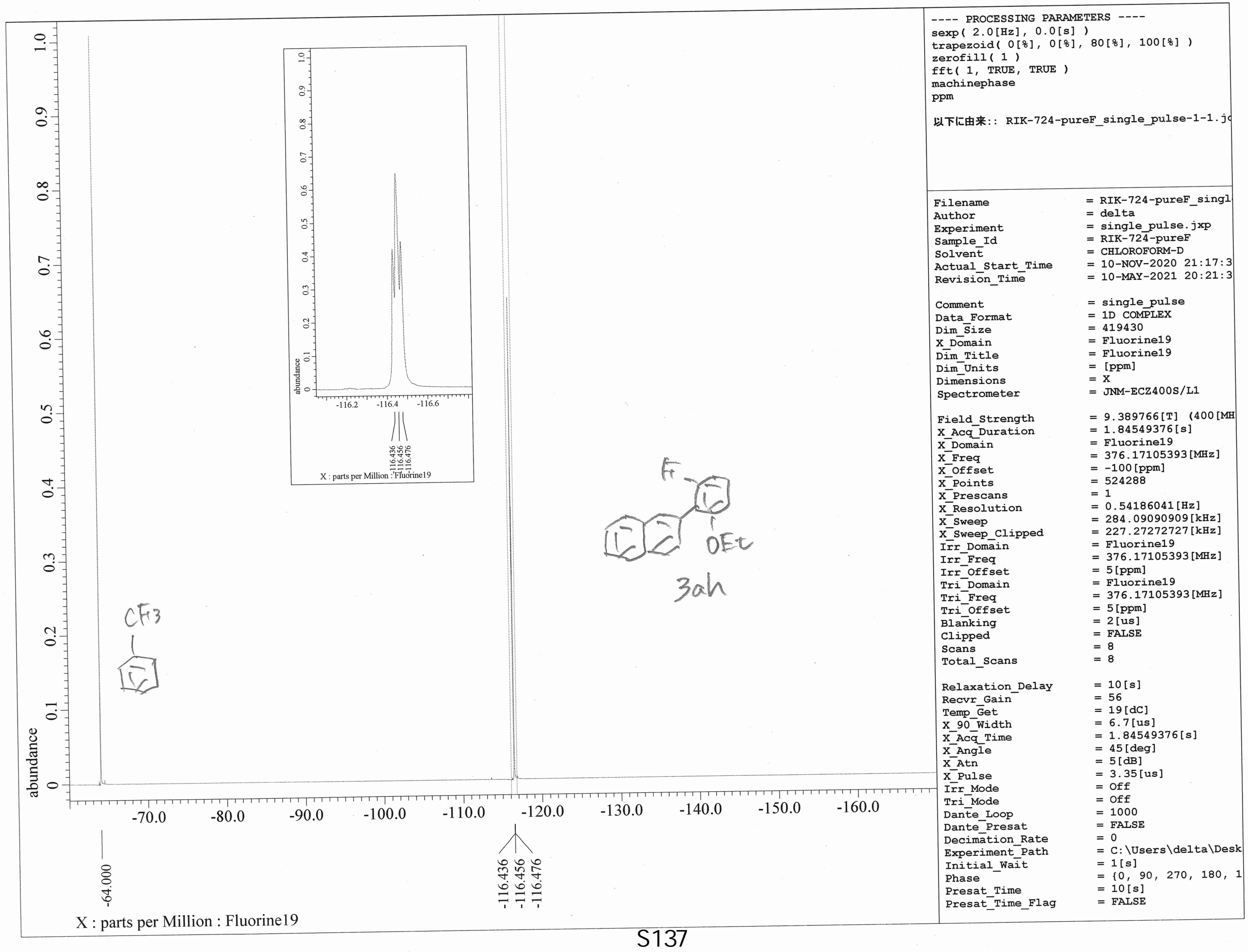




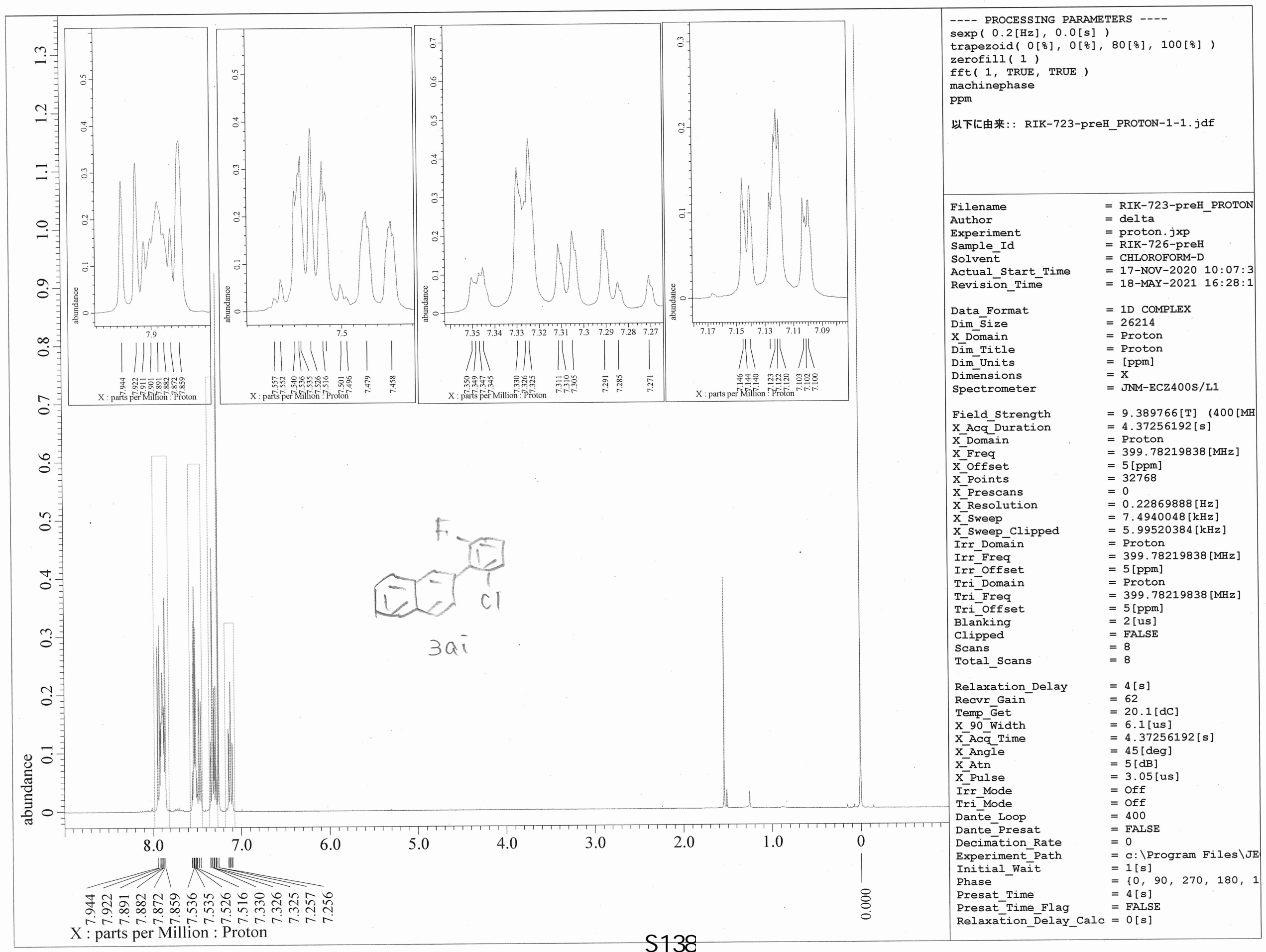




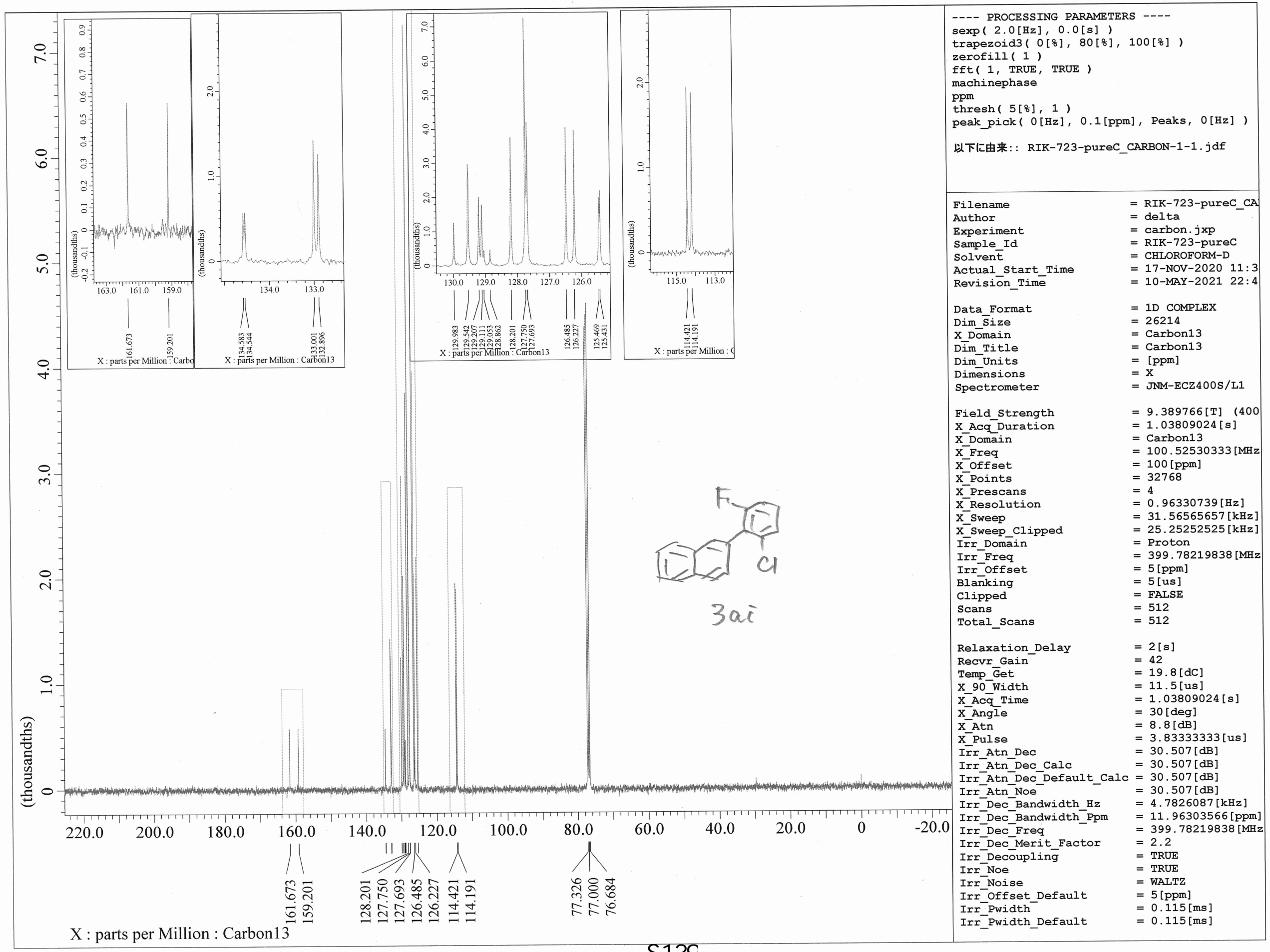




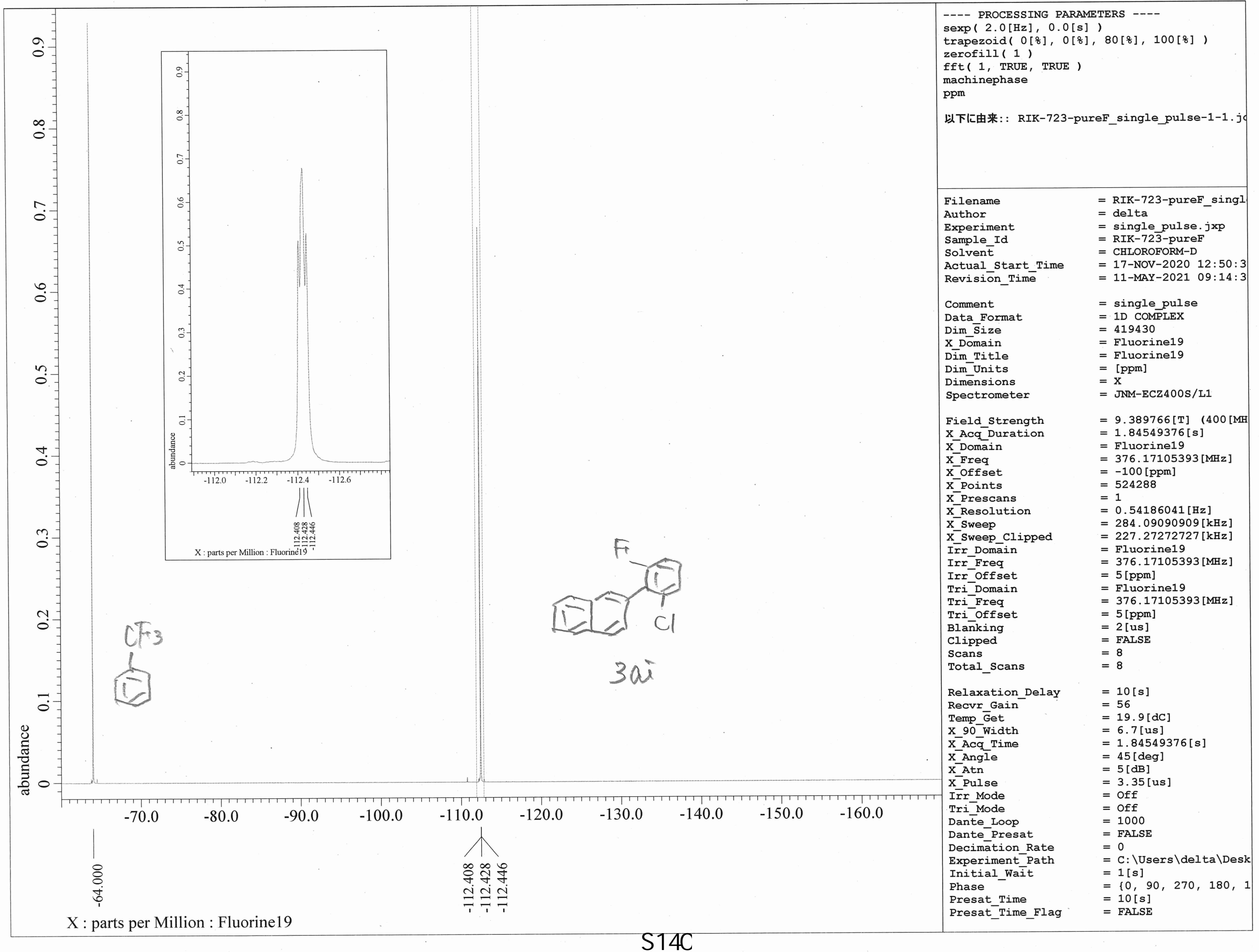




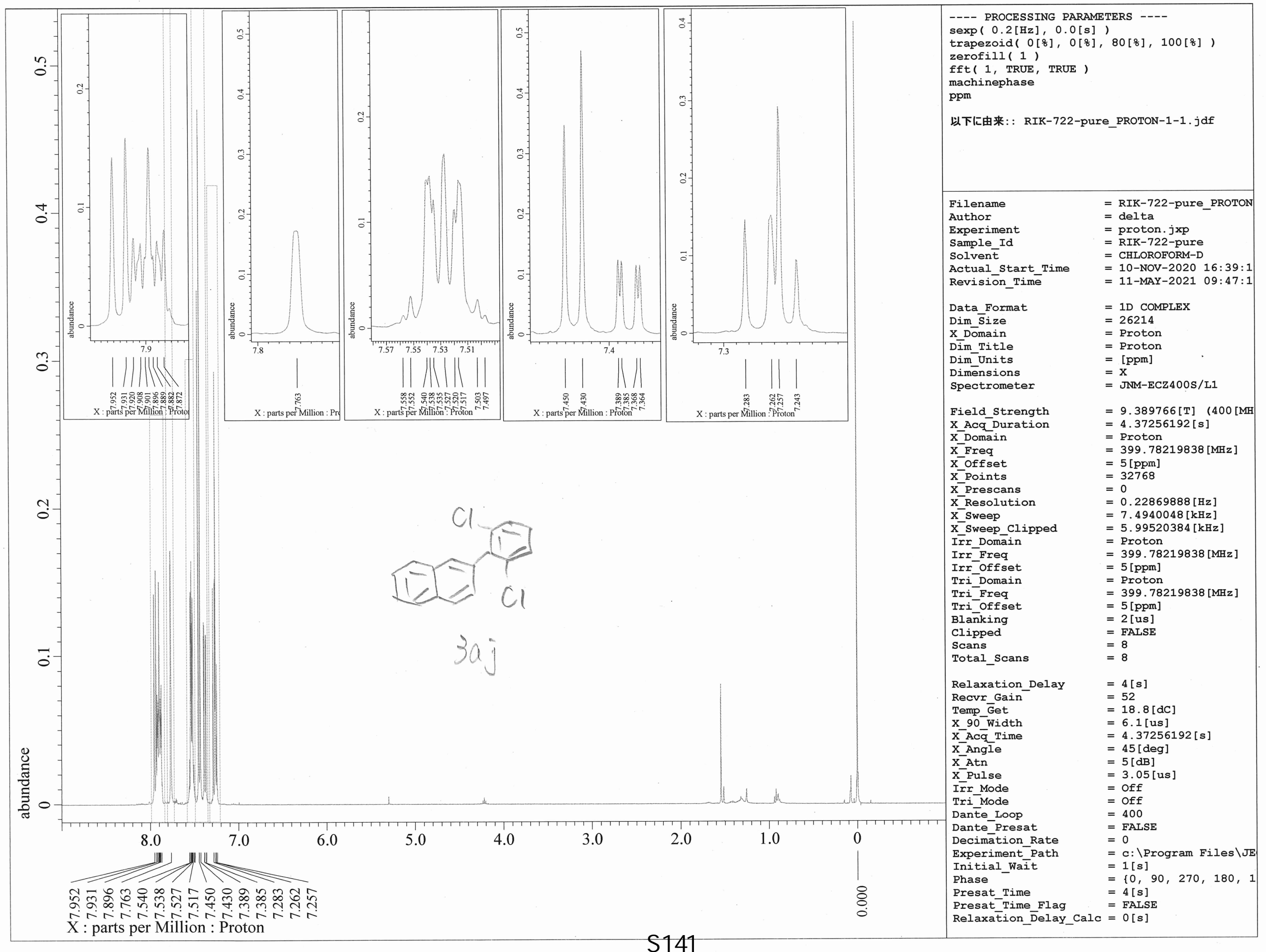




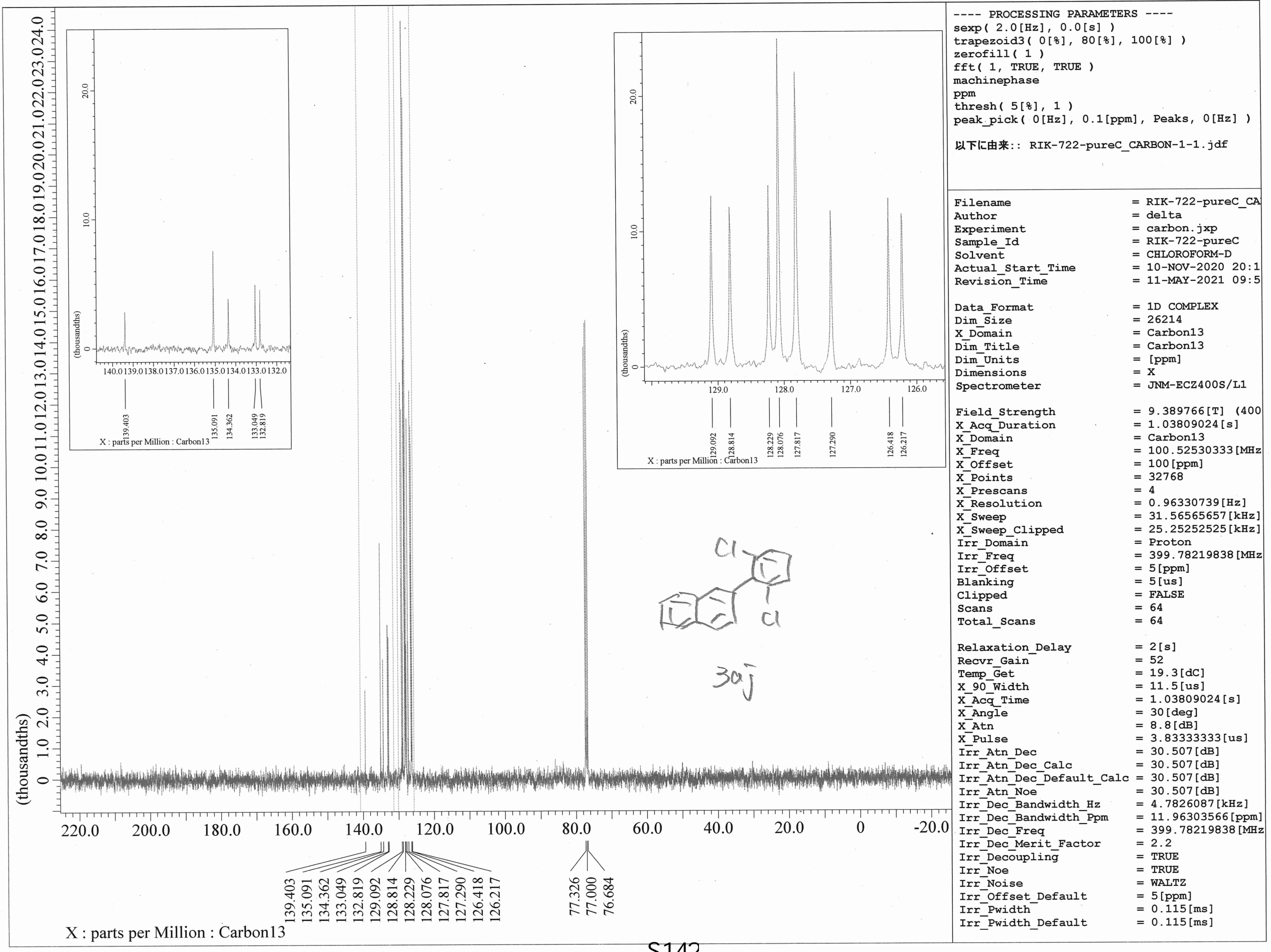




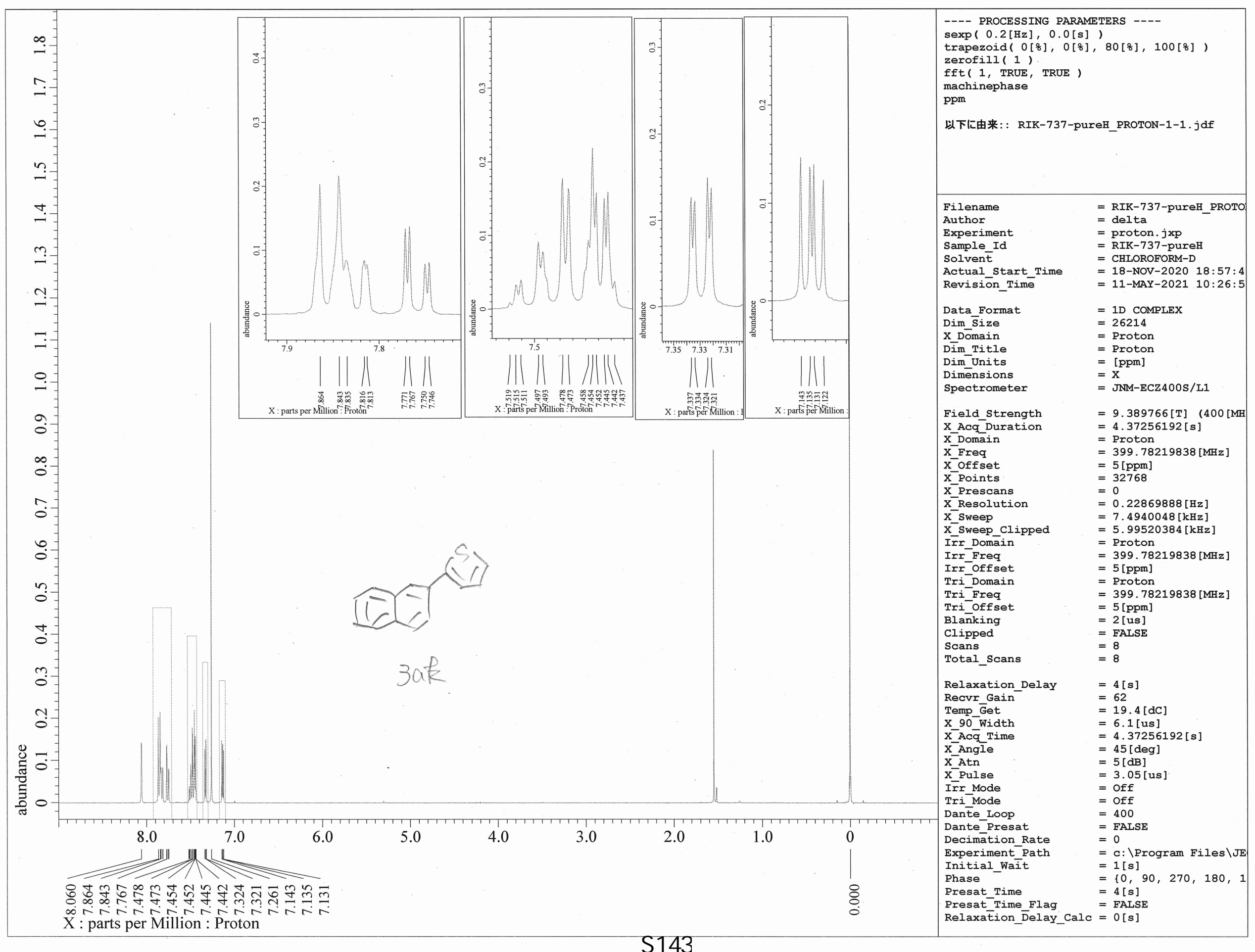




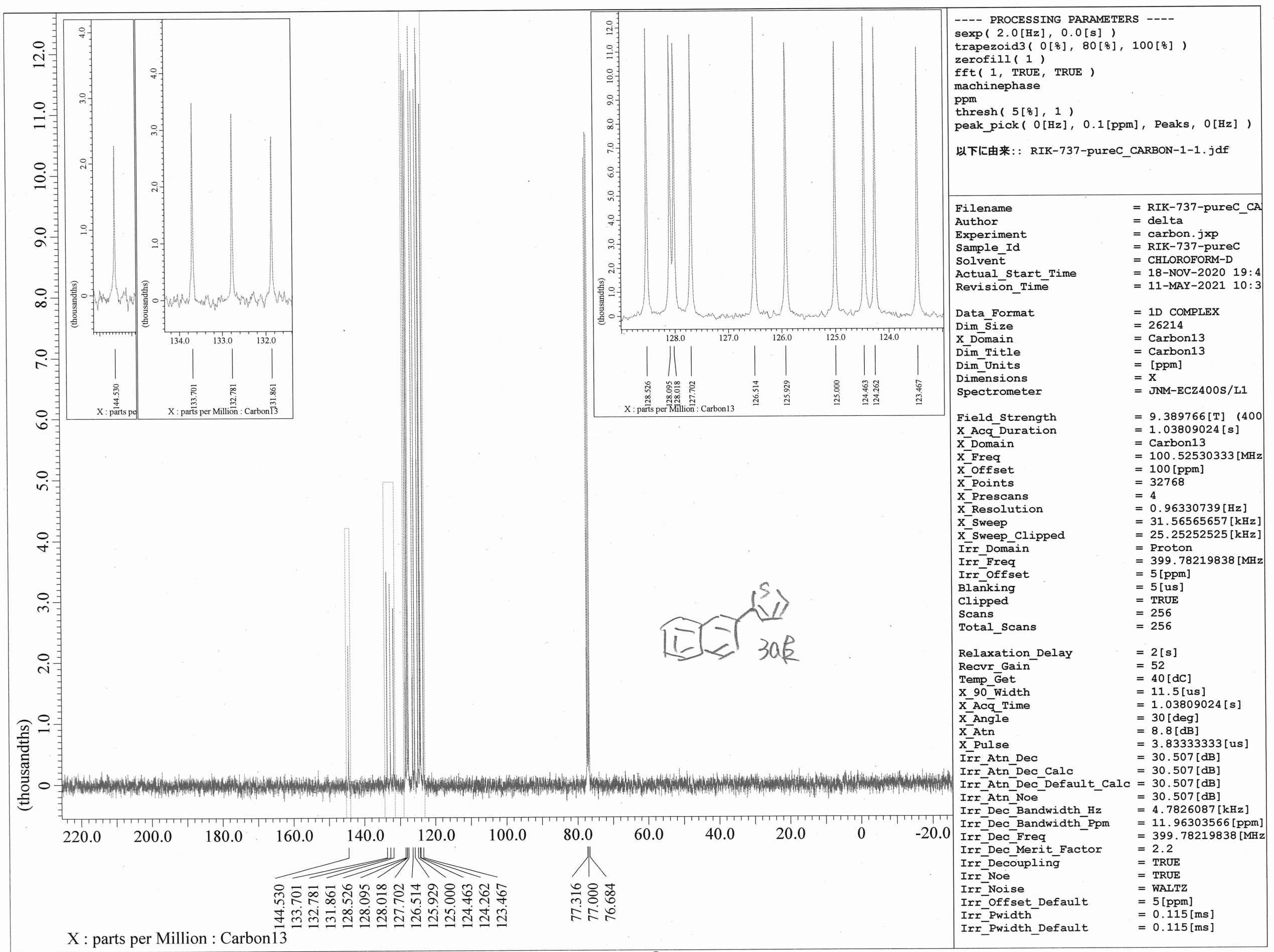




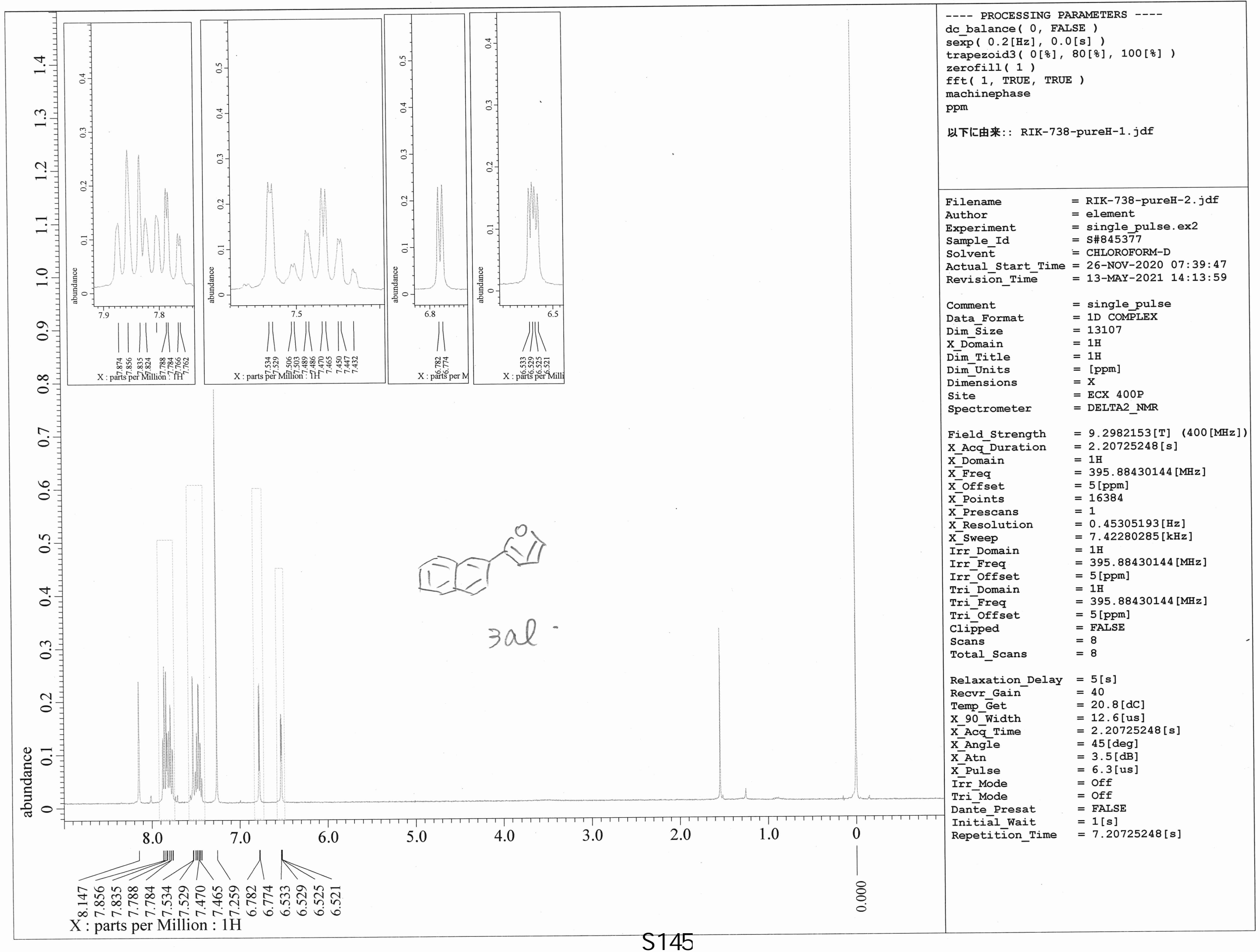




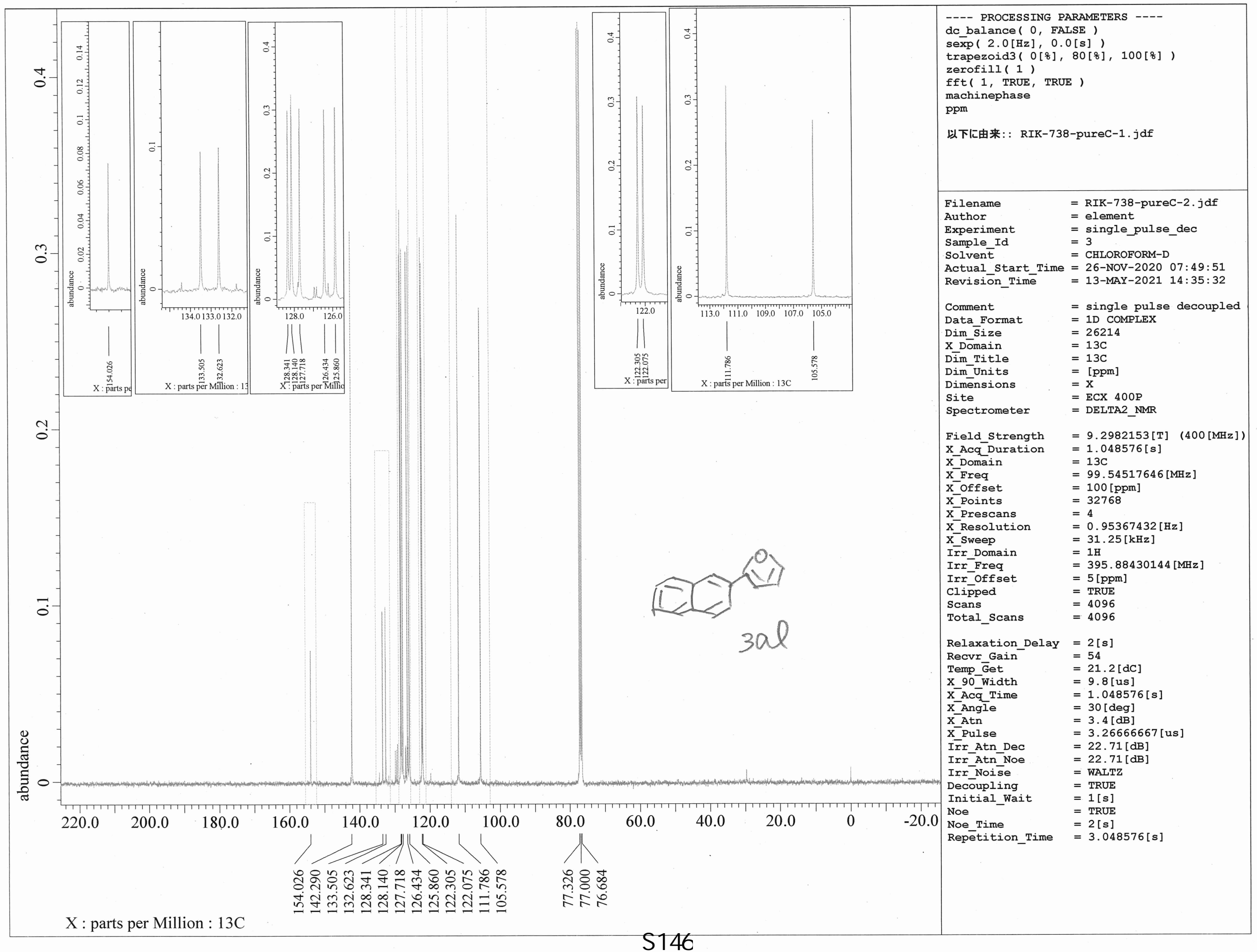




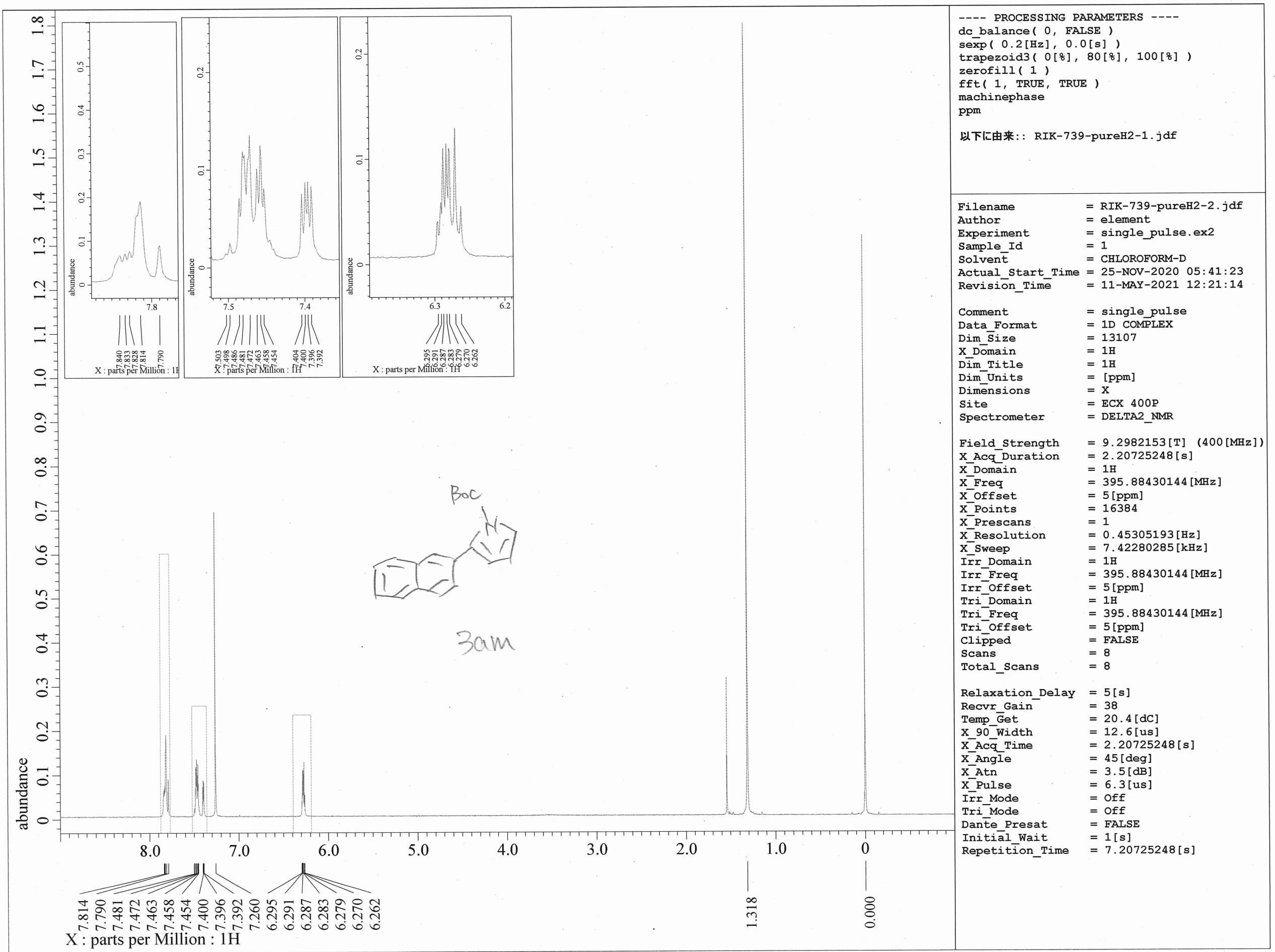




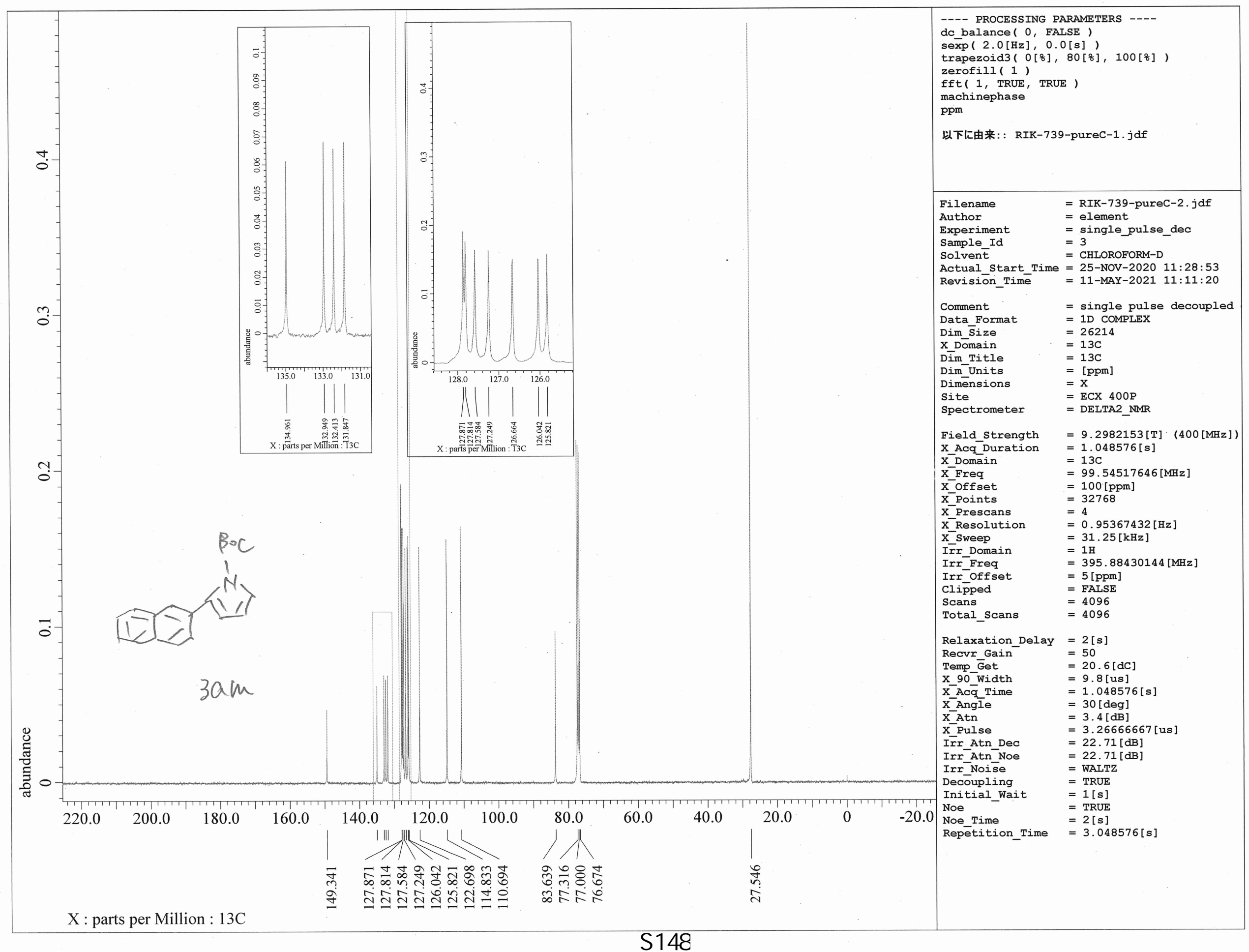




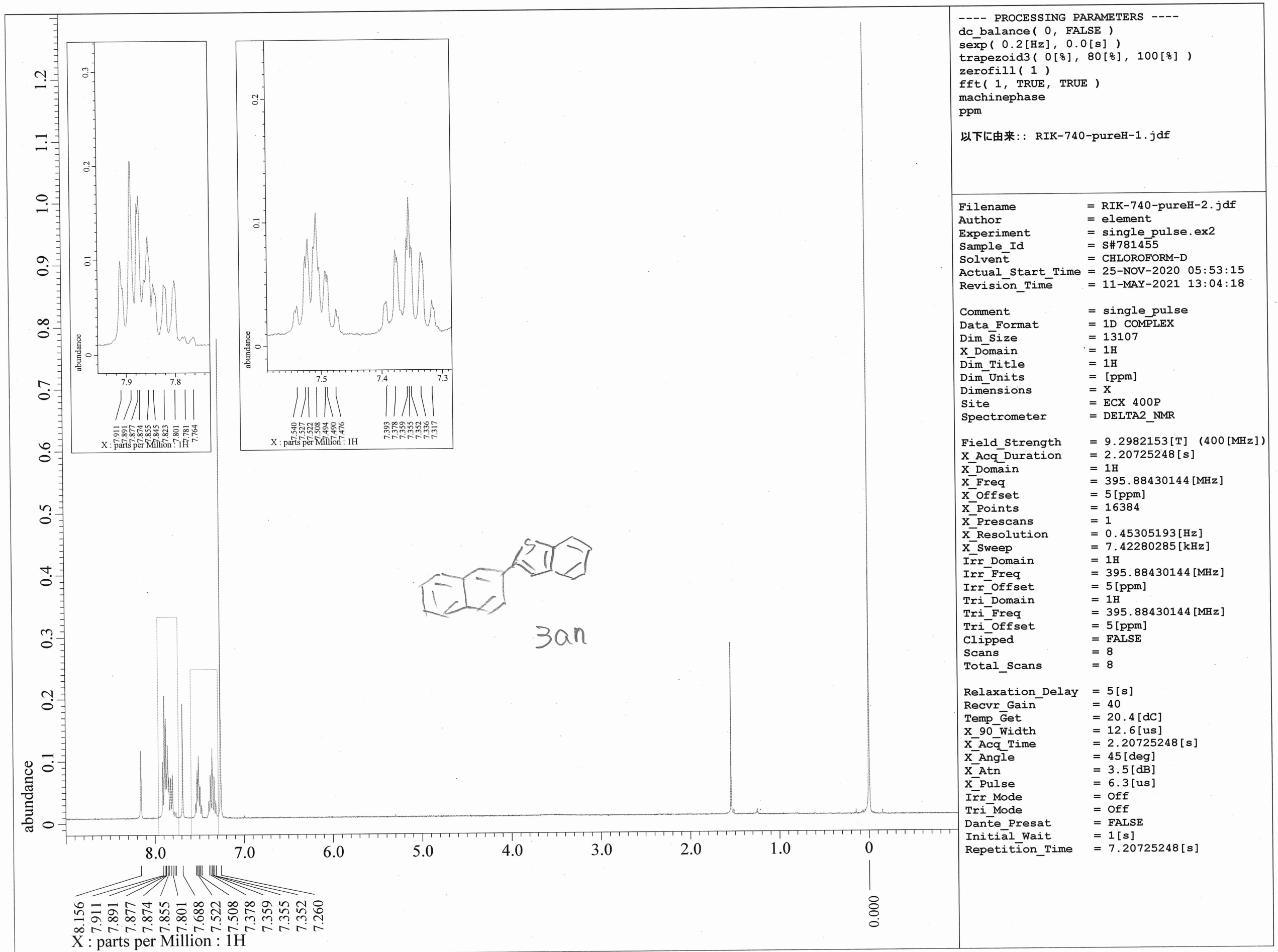




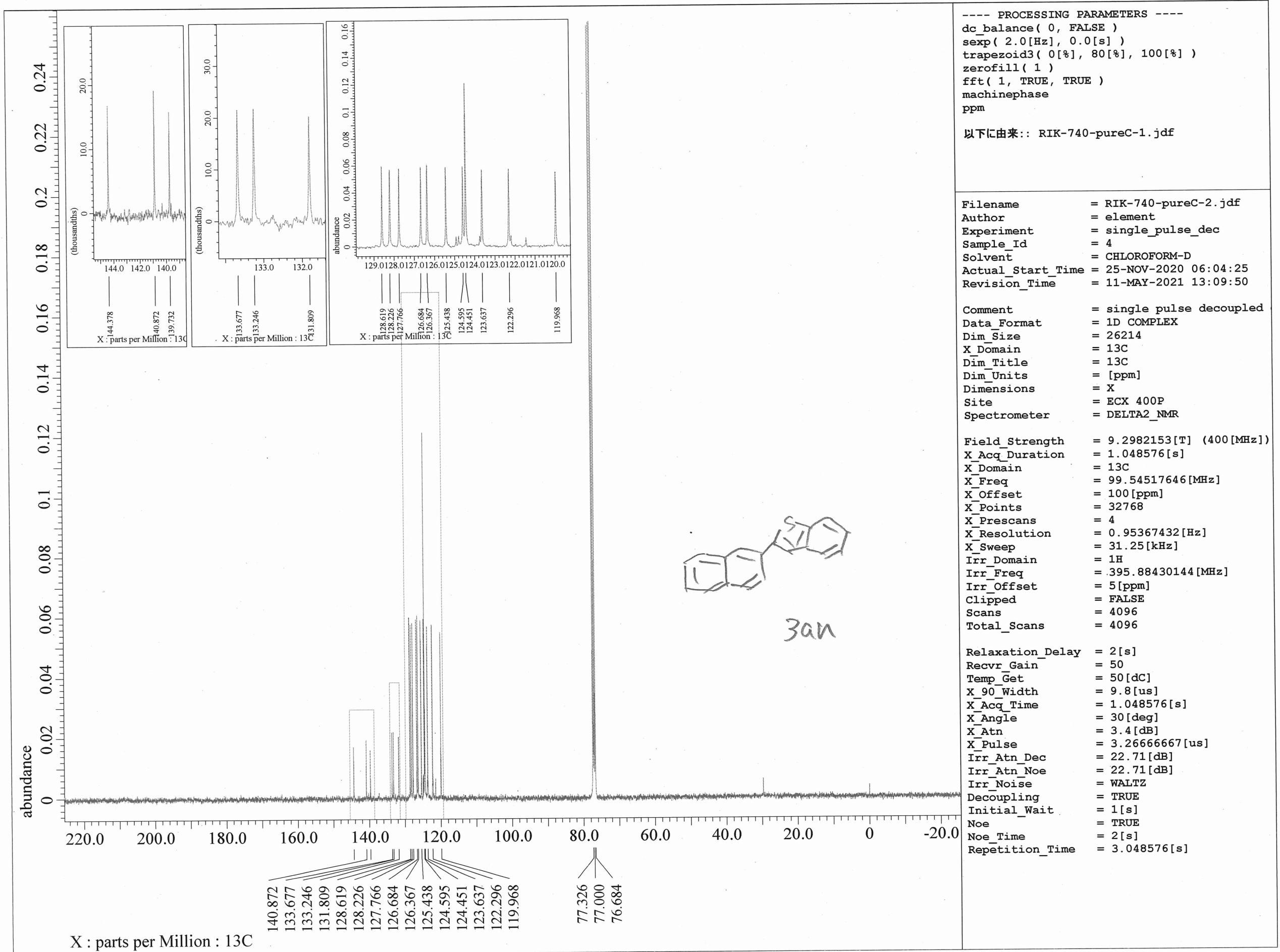




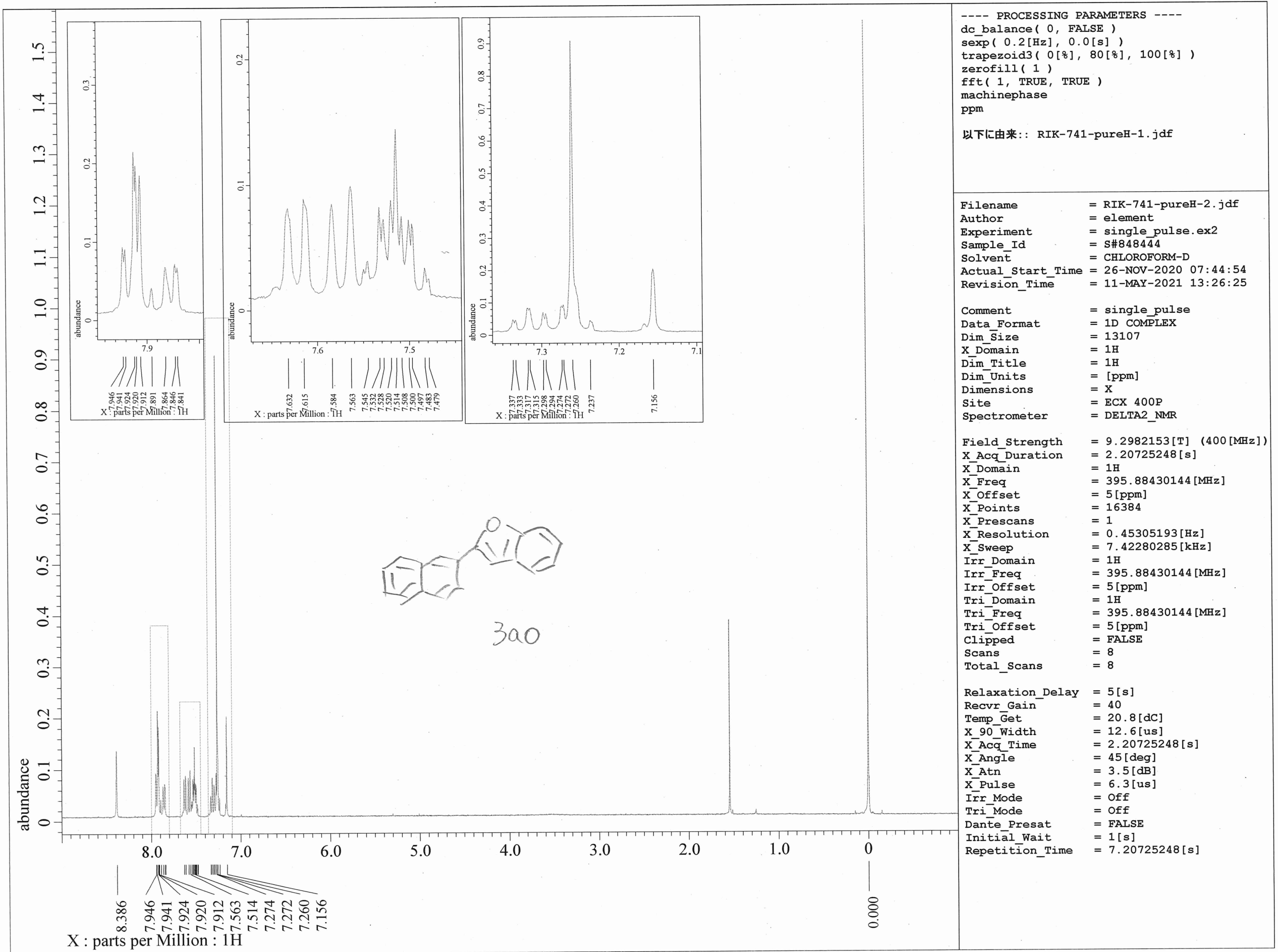

S151 


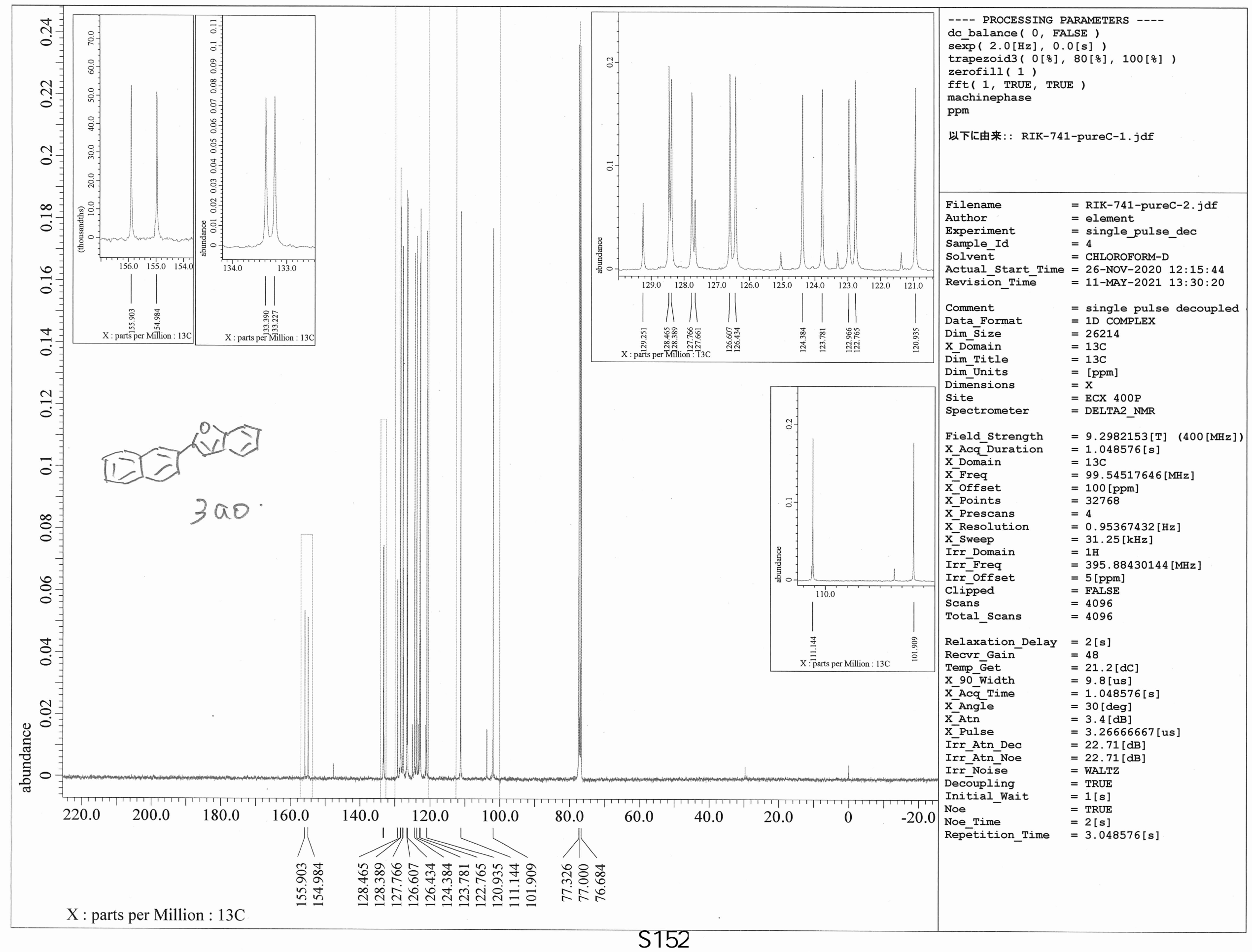

